Technische Universität München

Max-Planck-Institut für Quantenoptik

\title{
Trapping and observing single atoms in the dark
}

\author{
Thomas A. Puppe \\ Vollständiger Abdruck der von der Fakultät für Physik \\ der Technischen Universität München \\ zur Erlangung des akademischen Grades eines \\ Doktors der Naturwissenschaften (Dr. rer. nat.) \\ genehmigten Dissertation.
}

Vorsitzender : $\quad$ Univ.-Prof. Dr. H. Friedrich

Prüfer der Dissertation :

1. Hon.-Prof. Dr. G. Rempe

2. Univ.-Prof. Dr. F. von Feilitzsch

Die Dissertation wurde am 23. 05. 2007

bei der Technischen Universität München eingereicht

und durch die Fakultät für Physik am 23.07.2007 angenommen. 



\section{Abstract}

A single atom strongly coupled to a single mode of a high-finesse cavity is the principal system of matter-light interaction. Experimental studies of fundamental effects in this system require a reliable localization of the atom in the cavity mode.

This thesis reports the realization of a novel blue-detuned intracavity dipole trap. The blue trap combines the perfectly aligned, high-contrast modes of the high-finesse cavity to form a potential landscape in which an atom is stored close to a dark center, where the Stark shift vanishes. As a consequence, the free-space properties of the confined atom are largely retained, while it is well isolated by the surrounding repellant blue light. The flexibility to individually tailor the radial and axial confinement enables efficient loading. Cavity cooling is used to reliably prepare strong coupling.

The performance of the blue trap is demonstrated by spectroscopy of the normal modes of the coupled system. Good localization in a region of strong coupling and a Stark shift below the atomic linewidth are deduced from the spectrum by comparison with the analytical theory. Moreover, the preserved large atom-cavity detunings implement the dispersive regime, where the presence of the atom is detected while it spontaneously scatters only about one photon. Hence, single atoms are trapped and observed in the dark. Since strong cavity-induced heating can be avoided, the blue trap stores atoms in a parameter regime compatible with three-dimensional cavity cooling, which can increase storage times by orders of magnitude.

A first application of the intracavity dipole trap is the spectroscopy of the JaynesCummings ladder. The vacuum-Rabi splitting for a single trapped atom is a direct proof for strong coupling and can be fully explained by semiclassical theory. In contrast, the splitting of the higher doublets is a distinct signature of field quantization. A first observation of two-photon excitation to the second doublet using bichromatic spectroscopy was enabled by the blue trap. This illustrates the potential of the blue intracavity dipole trap for the study of fundamental quantum effects.

An impressive feature of the strong coupling regime is the ability to infer the spatial position of a single atom from the cavity transmission. Single atom transits are observed with an experimental adaption of the atomic kaleidoscope that uses a combination of higher-order modes to obtain position information in the transverse plane. 



\section{Contents}

$\begin{array}{ll}\text { Title } & 1\end{array}$

$\begin{array}{llr}1 & \text { Introduction } & 9\end{array}$

1.1 The strongly-coupled atom-cavity system . . . . . . . . . . . . . . . . . . . 10

1.2 The present work $\ldots \ldots \ldots \ldots \ldots \ldots \ldots$

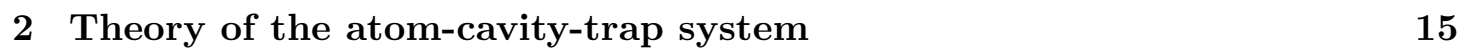

2.1 Jaynes-Cummings model . . . . . . . . . . . . . . . . . . . . . . . 16

2.2 Open quantum system $\ldots \ldots \ldots \ldots \ldots \ldots \ldots$

2.2 .1 Quantum regression theorem . . . . . . . . . . . . . . . 23

2.3 Atomic motion and light force . . . . . . . . . . . . . . . . . . 23

$2.3 .1 \quad$ Force operator . . . . . . . . . . . . . . . . . . . 24

2.4 Low-excitation limit $\ldots \ldots \ldots \ldots$

2.5 Momentum diffusion . . . . . . . . . . . . . . . . . . . . . . . 26

2.6 Velocity-dependent forces $\ldots \ldots \ldots \ldots \ldots$. . . . . . . . . . . . . . 27

2.7 Intracavity dipole trap $\ldots \ldots \ldots \ldots \ldots \ldots \ldots \ldots$

\begin{tabular}{|lll}
3 & The idea of the blue intracavity dipole trap & 31
\end{tabular}

3.1 Intracavity dipole traps $\ldots \ldots \ldots \ldots \ldots \ldots \ldots$

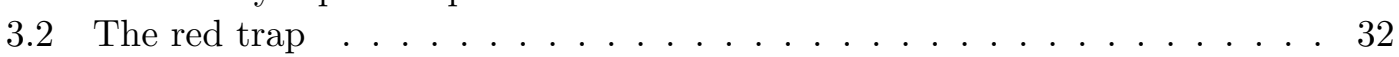

3.3 The blue trap . . . . . . . . . . . . . . . . . . . . . . . . . . 33

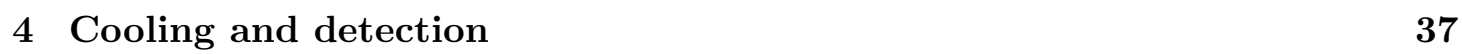

4.1 Cavity cooling. . . . . . . . . . . . . . . . . . . . . . . . . . 37

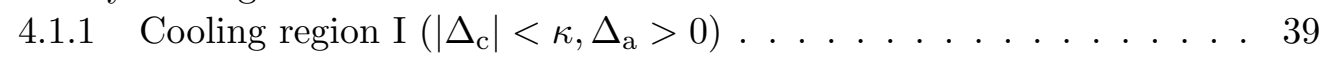

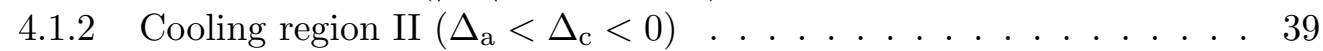

4.2 Resonant and off-resonant detection $\ldots \ldots \ldots \ldots$. . . . . . . . . 40

4.3 Cooling regions and Stark shift $\ldots \ldots \ldots$. . . . . . . . . . 40

\begin{tabular}{|lll}
5 & Numerical simulation & 43
\end{tabular}

5.1 Background . . . . . . . . . . . . . . . . . . . . . . . . . . 43

5.2 Algorithm . . . . . . . . . . . . . . . . . . . . . . . . . . 44

5.3 Results $\ldots \ldots \ldots \ldots \ldots \ldots \ldots \ldots$

$5.3 .1 \quad$ Cooling region I $\left(\left|\Delta_{\mathrm{c}}\right|<\kappa, \Delta_{\mathrm{a}}>0\right) \ldots \ldots . \ldots . \ldots 46$ 
5.3 .2 Cooling region II $\left(\Delta_{\mathrm{a}}<\Delta_{\mathrm{c}}<0\right) \ldots \ldots \ldots \ldots$. . . . . . . 50

5.3 .3 Sample trajectory $\ldots \ldots \ldots \ldots \ldots$. . . . . . . . . 54

$\begin{array}{lll}6 & \text { Experimental setup } & 57\end{array}$

$6.1 \quad$ Magneto-optical trap and atomic fountain . . . . . . . . . . . . . . . 58

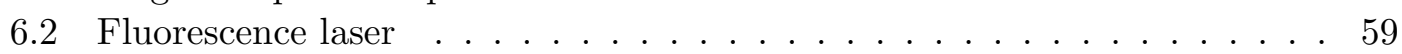

6.3 Optical pumping beam $\ldots \ldots \ldots \ldots \ldots$. . . . . . . . . . . . . 60

6.4 High-finesse cavity $\ldots \ldots \ldots \ldots$. . . . . . . . . . . . . . 6 60

6.5 Laser system . . . . . . . . . . . . . . . . . . . . . . . . . 62

6.5 .1 Dipole laser stabilization . . . . . . . . . . . . . . . . . 64

6.5 .2 On-axis cavity excitation . . . . . . . . . . . . . 6 65

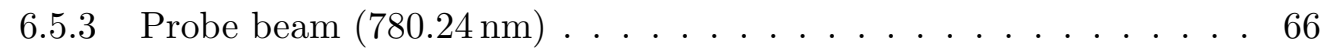

$6.5 .4 \quad$ Red trap and stabilization laser $(785.2 \mathrm{~nm}) \ldots$. . . . . . . . . 66

6.5 .5 Axial confinement laser: pancakes $(772.5 \mathrm{~nm}) \ldots \ldots 6$

6.5.6 Transverse guiding \& trapping: funnels \& doughnut $(775.2 \mathrm{~nm})$. . 66

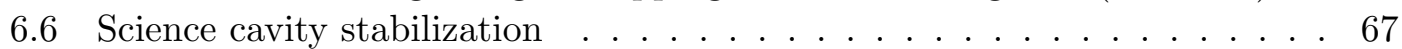

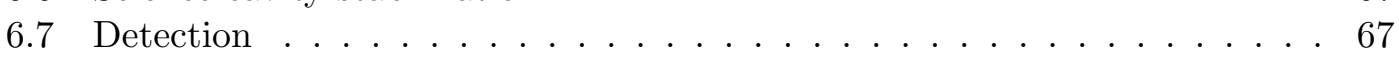

6.8 Data acquisition $\ldots \ldots \ldots \ldots \ldots \ldots$

\begin{tabular}{|lll}
\hline 7 & Experimental realization of the blue trap & $\mathbf{7 1}$
\end{tabular}

7.1 Blue-detuned modes for guiding and trapping . . . . . . . . . . . . . . . . 71

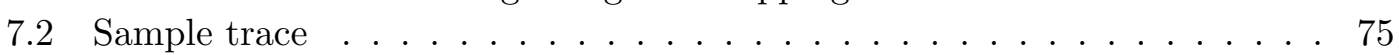

7.3 Sample trace for blue only trap $\ldots \ldots \ldots \ldots$. . . . . . . . . . 75

7.4 Normal-mode splitting . . . . . . . . . . . . . . . . . . . . . . . . 77

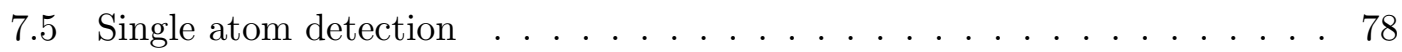

7.5 .1 Resonant dispersive detection . . . . . . . . . . . . . 80

$7.5 .2 \quad$ Poisson analysis $\ldots \ldots \ldots \ldots$. . . . . . . . . . . . . 81

7.6 Qualification . . . . . . . . . . . . . . . . . . 83

7.7 Velocity-dependent forces $\ldots \ldots \ldots \ldots$. . . . . . . . . . . 85

7.8 Cavity cooling in the blue trap . . . . . . . . . . . . . . . . 86

8.9 Towards three-dimensional cavity cooling in the blue trap . . . . . . . . . 88

7.10 Conclusions and prospects $\ldots \ldots \ldots \ldots \ldots$. . . . . . . . . . . . . 89

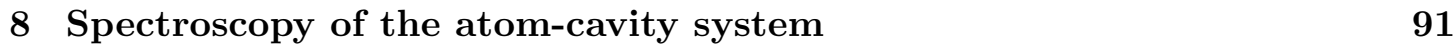

$8.1 \quad$ Analytical concept . . . . . . . . . . . . . . . . . . . . 92

8.2 Normal-mode splitting . . . . . . . . . . . . . . . . . . . . . . . . 94

8.3 Theoretical analysis of the two-photon spectroscopy $\ldots$. . . . . . . . . . . 97

8.3 .1 Model systems . . . . . . . . . . . . . . . . . . . 9 97

8.3 .2 Spectroscopy of the atom-cavity system . . . . . . . . . . . . . 98

8.4 Numerical simulations . . . . . . . . . . . . . . . . . . . . 100

$8.4 .1 \quad$ Algorithm . . . . . . . . . . . . . . . . . . . . 100

8.4 .2 Bichromatic spectroscopy of the Jaynes-Cummings ladder . . . . . 101

8.5 Experimental two-photon spectra . . . . . . . . . . . . . . . . 101

8.5 .1 Two-photon spectrum in the red trap . . . . . . . . . . . 103 
8.5 .2 Two-photon spectrum in the blue trap . . . . . . . . . . . . . 105

$\begin{array}{llr}9 & \text { Kaleidoscope } & 107\end{array}$

$9.1 \quad$ Higher order modes . . . . . . . . . . . . . . . . . . . . . . . . . 107

9.2 Idea of the kaleidoscope $\ldots \ldots \ldots \ldots$. . . . . . . . . . . . . 108

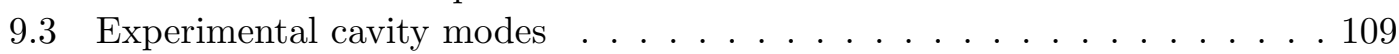

9.4 Transits through $\mathrm{TEM}_{10}+\mathrm{TEM}_{01} \ldots \ldots \ldots \ldots$. . . . . . . . . 111

9.4 .1 Transits through $\mathrm{TEM}_{10} \ldots \ldots \ldots \ldots \ldots$. . . . . . . . . . . . . . . . . . .

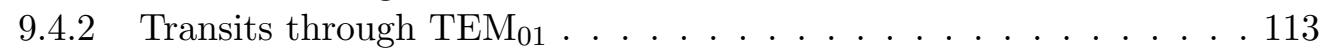

9.4 .3 Simultaneous transits through $\mathrm{TEM}_{01} \& \mathrm{TEM}_{10} \ldots \ldots . \ldots 113$

9.5 Atom in blue-detuned laser fields . . . . . . . . . . . . . . . . . 115

\begin{tabular}{lr}
\hline 10 Outlook & 117
\end{tabular}

\begin{tabular}{|l|l}
\hline A Rubidium energy levels & 121
\end{tabular}

\begin{tabular}{|lr}
\hline B Parameters in the numerical Simulations & 123
\end{tabular}

\begin{tabular}{|l|l|l|}
\hline C Polarization of the high-finesse cavity modes & 125
\end{tabular}

\begin{tabular}{lr}
\hline Bibliography & 129
\end{tabular}

\begin{tabular}{lr}
\hline Publications & 143
\end{tabular}

\begin{tabular}{lr}
\hline Danksagung & 145
\end{tabular} 



\section{Chapter 1}

\section{Introduction}

Fundamental quantum effects are expected to be observed for systems with only a few relevant states and are generally susceptible to the coupling to an environment, which destroys the coherent evolution (11). The success of quantum mechanics to correctly predict and describe experimental results has long been accompanied by a controversy about the philosophical difficulties in the understanding of the establishment of a concrete measurement outcome, referred to as the 'quantum measurement problem' (2). Up to now, this problem could be largely ignored in favor of a pragmatic view, because in experiments the outcome of the repeated measurements is well described by the statistical prediction of an ensemble average (3). For a finite system dissipation is largely accepted as the mechanism to explain the appearance of the classical world in quantum mechanics (4; 5). In recent years repeated measurements on single quantum systems as well as macroscopic quantum systems have become technologically feasible. Particularly interesting are open quantum systems that can be effectively monitored via their decay channel (6). Here, also the back action of a measurement and the evolution under continuous (incomplete) measurements play a role $(7,8,9)$. This is particularly apparent in quantum feedback on an individual system (10), because a successful correction relies on an accurate prediction of the influence of the measurement on the system. Quantum feedback allows to establish a desired target state (11, 12), e.g., to realize spin-squeezing (13, 14) and adaptive quantum measurements of the optical phase (15; 16). Feedbackmediated quantum measurement at the fundamental quantum limit has recently been studied on coherent states of a photon field (17). To further explore fundamental quantum mechanics, experimental research depends upon suitable, well isolated laboratory systems prepared by external control.

In quantum optics the preparation of quantum systems largely relies on laser cooling and trapping methods $(18,19,20)$. The field was initiated by laser cooling techniques $(21 ; 22$; 23, 24) which have first been realized in ion traps $(25 ;, 26)$ and later for neutral atoms (27). Cold samples of neutral atoms have been prepared in magnetic traps (28), magneto-optical traps (29, 30) and dipole traps (31). Sub-Doppler temperatures have been achieved in optical molasses (32) with polarization-gradient cooling (33; 34) and velocity-selective coherent population trapping (35). These systems were further devel- 
oped to the single particle level $(36 ; 37 ; 38 ; 39)$ and to manipulate the quantum state of single (40) or small sets of particles $(41$; 42 ; 43). However, the external control imposes a significant modification to the system under study. This problem is illustrated by high precision experiments, like atomic clocks (44). While trapped systems have the potential for accuracies exceeding the free flight fountain experiments by orders of magnitude, they are limited by unregulated clock shifts induced by the confinement. Hence, the ultimate goal is to eliminate the uncertainty in the influence of the external control.

\subsection{The strongly-coupled atom-cavity system}

A single atom strongly coupled to the mode of a high-finesse cavity constitutes a fundamental combined quantum system of matter-light interaction (45). It consists of two different, well understood subsystems: a single two-level atom and a single mode of the electromagnetic field described by a quantum-harmonic oscillator. In the strong-coupling regime the coherent interaction between the atom and the mode exceeds the decay rates of the cavity mode and the atomic polarization. The experimental challenge to realize this system is to achieve strong constant coupling over a sufficiently long interaction time with good isolation from the environment. Pioneering experiments with Rydberg atoms were enabled by microwave cavities at cryogenic temperatures. The implementation of the one-atom maser (46; 47) enabled the observation of quantum collapse and revival (48) and the vacuum-Rabi oscillation (49) for many atoms. Strong coupling in the optical domain only became feasible with the development of high-quality dielectric coatings (50). A coupling exceeding the decay rates is demonstrated by the observation of a normal-mode splitting. The normal-mode splitting was studied for many atoms in a beam acting as an 'effective atom' (51; 52), realized for a single atom on average $(53,54)$ and a single transiting atom (55).

The experiments in the microwave and optical regimes complement each other. The field inside the microwave cavity is manipulated and observed by the Rydberg atoms passing the mode (56) since the atomic state can be detected with very high efficiency. By now, many of these deliberate interactions are possible before the cavity field decays. A characteristic example for experiments in the microwave regime is the recent observation of quantum jumps in the photon field (57) by a sequence of quantum nondemolition (QND) measurements (58). This advancement should allow to further explore the quantum-to-classical boundary (59) by, e.g., analyzing the decoherence of mesoscopic entangled states involving an increasing number of particles ( $\underline{60})$ or testing complementarity with an interferometer (61). In contrast, in the optical regime the photons emitted from the cavity are detected with high efficiency and provide continuous observation of the system. Because the emitted photons can easily be distributed via optical fibres, driving applications in the optical domain include the realization of deterministic single photon sources $(62,63,64,65,66)$ for linear optical quantum computation (67) and a matter-light interface for quantum networks (68, 69; 70) to reversibly map the quantum state of an atom onto a photon (68, 65).

Solid state systems fundamentally solve the problem of localization. A fixed strong 
coupling is achieved by a sophisticated production process. The vacuum-Rabi splitting has been measured for a cooper pair box coupled to a superconducting coplanar waveguide resonator (71), a flux qubit (72) coupled to a superconducting resonator, and in the optical regime for single quantum dots coupled to a photonic crystal (73; 74), a micro-pillar (75) and a microdisk cavity (76).

From the very beginning of cavity QED the question was raised whether distinct quantum effects could be observed, but as Jaynes and Cummings state '... the prospects of detecting such a difference are extremely dubious, for we will see that the semiclassical theory actually reproduces many of the features which one commonly supposes can be found only with field quantization' (45). This holds true for the vacuum-Rabi splitting observed in this experiment which is the signature of the excitation to the first doublet of states in the Jaynes-Cummings ladder. The vacuum-Rabi splitting can be fully explained by the semiclassical theory where an atom exchanges energy with a cavity mode. It is accounted for by Bloch equations, the analytical solution in the low excitation limit as well as by linear-dispersion theory (51). In contrast, the spectroscopy of the higher lying doublets would constitute an immediate test for nonperturbative cavity QED (77). Conversely, these resonances do not occur in the low saturation theory at all. Experimental evidence has been observed in the fourier components of the Rabi oscillations (78) in the microwave regime. A direct spectroscopic measurement is strongly anticipated and a major goal for experiments where the normal-mode splitting has been observed (79). A first signal of a two-photon resonance was reported in a quantum dot system (80), but a different explanation of a similar three peak structure exists (74). The photon blockade is a closely related effect which has been observed in an atom-cavity system (81). Experimental observation of quantum effects become feasible with the progress in the experimental control in the preparation of the system.

\subsection{The present work}

In the context of this thesis, the atom-cavity system was extended by a far-detuned intracavity dipole trap (82) to achieve good localization of the atom. Compared to trapping in the near-resonant mode $(83$; 84$)$ where the motion is driven by strong dipole force fluctuations $(85,86)$ and photon recoils due to spontaneous emission, the fardetuned dipole trap provides a near conservative potential. The oscillatory motion in this conservative potential of the dipole trap has been observed and the storage times achieved are about an order of magnitude longer than in the near-resonant case (87). The reddetuned intracavity dipole trap allowed for the first observation of cavity cooling (88). In contrast to all standard free-space laser cooling techniques which rely on spontaneous emission from the atom to remove energy and entropy from the system, cavity cooling does not rely on the excitation of the atom. Instead the excitation is transferred via the contribution of the cavity state to the normal modes and the blue-detuned photons emitted from the cavity remove kinetic energy from the coupled atom (89, 90, 91). Cavity cooling was demonstrated to be at least five times more efficient than standard free-space techniques at equal atomic excitation (88). Because cavity cooling does not 
rely on repeated excitation cycles, it has the potential to make new systems accessible to laser cooling, e.g. molecules (92) or atoms carrying a quantum bit (93).

The measurement of the vacuum-Rabi splitting of a single trapped atom-cavity system was made possible by the combination of the red dipole trap with repeated cavity cooling to restore good coupling (94). In the red-detuned dipole trap the atom is stored close to an intensity maximum where the atomic detuning is modified by the AC Stark shift. The variation of the atom-cavity detuning by the trap depth allowed to scan through the anticrossing of the normal modes, but restricts the feasible detuning regimes and trap depths. A red-detuned dipole trap at the 'magic wavelength' for Cs (95) stores atoms without a differential energy shift: including the coupling to higher lying electronic states, the Stark shift induced on the excited state exactly matches the ground state shift. Since a dipole trap at a magic wavelength relies on a special level scheme it is only possible for some atoms. For rubidium the red magic wavelength is experimentally impracticable.

In this thesis, a more fundamental approach to remove the influence of the trap on the stored particle is realized by blue-detuned intracavity dipole trap (96). The highfinesse cavity provides perfectly aligned modes. A combination of higher-order modes is used in the blue intracavity dipole trap to guide and store single atoms in regions of strong coupling to the near-resonant mode. The close-to-perfect visibility of the standing wave creates a trap center that is accurately dark. The blue trap has a number of advantages for the preparation of a strongly coupled atom-cavity system. The atom is stored close to a dark center, where the Stark shift vanishes, completely surrounded by blue light. Since the trap height does not contribute to the Stark shift, much higher confinement can be realized. Moreover, since the free-space detuning of the atom is preserved during the loading, there is no restriction to the accessible parameter regimes and strong cavity-induced heating $(85,86)$ can be avoided. Because the atom is repelled from the blue-detuned light it can be loaded by creating a two-dimensional funnel. Slow atoms are guided to the trap center and captured without increase in energy. Weakly coupled atoms that are not collected by the funnel are rejected. An atom inside the trap is well isolated by the surrounding potential barrier, outside atoms are repelled.

The advantages of the blue trap are demonstrated by normal-mode spectroscopy. Good localization of the atom is restored and qualified by intervals of cavity cooling. The normal-mode spectrum of the strongly coupled atom-cavity system prepared in the blue trap is well described by the analytical theory in the limit of low excitation. The Stark shift is found to be smaller than the atomic linewidth even for high potential barriers of blue light. In contrast to the red-detuned trap, the large bare detuning between atom and mode is preserved. This allows the dispersive detection of the presence of a single atom in $10 \mu$ s with $95 \%$ probability for a correct decision, while the atom scatters only about one photon during that time. Dispersive detection is complementary to cavity cooling in the sense that both mechanisms do not rely on the excitation of the atom, but use the cavity-like normal mode instead. Moreover, atoms are stored in the blue trap in a parameter regime that is compatible with three-dimensional cavity cooling, which is shown to extend the storage times by several orders of magnitude (97). 
Normal-mode spectroscopy of the Jaynes-Cummings ladder is a milestone in cavity QED. The observation of the vacuum-Rabi splitting, as the excitation to the first doublet, directly shows reliable strong coupling and is well described by the low excitation limit. In contrast excitations to the second doublet are not included in the semiclassical theory and are hence a direct observation of field quantization. The experimental observation requires a good control of the atom-cavity coupling. A proposal for an atomic beam experiment uses a 'hole-burning' technique to only address a certain class of atoms (77). In the present experiment a single atom is strongly confined and cavity cooling is used to restore and qualify for strong coupling. For this setting different schemes of mono- and bichromatic excitations are developed in this thesis. A first observation of a two-photon resonance in bichromatic spectroscopy is realized in the blue trap. This demonstrates the potential of the blue intracavity dipole trap for the anticipated fundamental studies of a single combined quantum system.

This experiment relies on the capability to detect a single atom (98, 99) to prepare a strongly-coupled atom-cavity system. The atom is trapped by switching the potential upon its detection. The strong influence of a single atom on the light field in the cavity given by the position-dependent coupling enables high-bandwidth detection with high spatial resolution (100, 101). It allows to implement feedback on the motion of the single atom (102). The 'atomic kaleidoscope' (103; 104) uses higher-order modes to obtain more detailed position information in the transverse plane. The transit of a single atom through higher-order modes is observed with a technique adopted to the experiment (105).

This thesis is organized as follows: Chapter2 introduces the theoretical background of the atom-cavity system and derives the analytical description in the low excitation limit. In Chap. 3, the idea of the blue intracavity dipole trap is developed and its advantages are discussed with special emphasis on two different parameter regimes of cavity cooling (Chap. 4). The analytical findings are supported by numerical simulations in Chap. 5 . After presenting the general experimental system in Chap. 6 the implementation of the blue trap and the experimental results are discussed in Chap. 7. The spectroscopy of the Jaynes-Cummings ladder is presented in Chap. 8. The atomic kaleidoscope is discussed in Chap. 9. An outlook puts the results of this thesis into a larger perspective (Chap. 10). 



\section{Chapter 2}

\section{Theory of the atom-cavity-trap system}

This chapter introduces the theory of the strongly-coupled atom-mode system as the fundamental system of matter-light interaction in cavity quantum electrodynamics (CQED). It is a combined quantum system of two different well understood subsystems: a single atom described by a two-level system and the light field of a single cavity mode is represented by a quantized harmonic oscillator (Fig. 2.1). The coupling between the atom and the mode is given by the dipole interaction. Neglecting the interaction with the environment leads to the well-known Jaynes-Cummings Model (45) discussed in section 2.1. The dipole interaction couples pairs of states with equal number of excitations, which therefore split into new normal modes called 'dressed-states' (33). The closed system is extended to an open system by including the decays of the subsystems into the vacuum modes (Sec. 2.2). At low excitation the master equation can be solved analytically for an atom at rest (Sec. 2.4). The strong-coupling regime defined by a coupling $g$ that exceeds both decay rates of the cavity field $\kappa$ and the atomic polarization $\gamma$ is characterized by well resolved normal modes. The position dependence of the coupling in the standing wave mode leads to strong light forces (Sec. 2.3). The emission of photons from the system is compensated by a weak probe beam. Since the cavity transmits photons into a well-defined mode they can be efficiently detected and allow for a continuous observation of the system. The dissipation also leads to strong velocity-dependent forces, both heating and cooling (Sec. 2.6). In addition to the free-space diffusion term which can be interpreted as a fluctuating dipole interacting with a mean field, the additional fluctuation of the intracavity field interacting with a mean atomic polarization contributes an extra term (Sec. 2.5). Both the velocity-dependent forces as well as the diffusion are particulary strong near the normal modes of the coupled system. Note that the normal modes and the forces can be derived in a classical model of dispersion and absorption of a massive, point-like dipole $(51,106)$. This is not the case for the diffusion, which is given by the correlations of the dipole-force fluctuations.

To take advantage of the system, the interaction time has to be much larger than the decay times. Therefore, atoms are trapped in far detuned intracavity dipole traps 


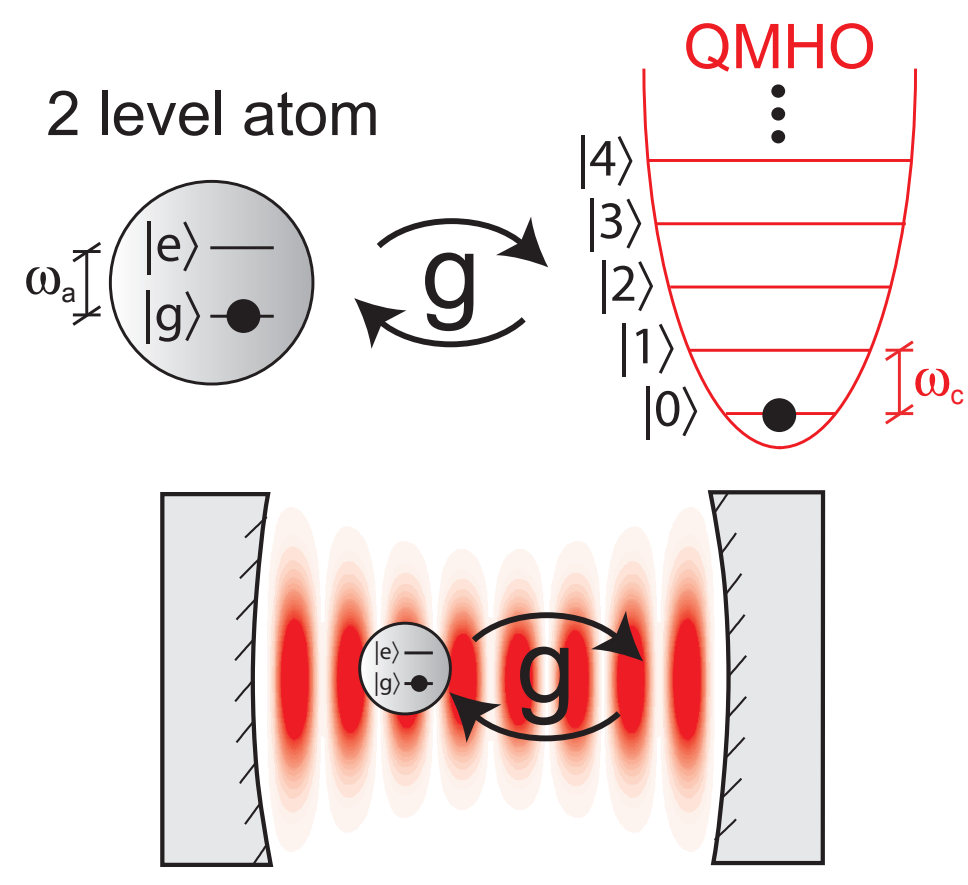

Figure 2.1: Strongly coupled atom-cavity system: A single two-level atom is coupled via the dipole interaction to the field of a single cavity mode described as a quantum harmonic oscillator (QMHO). The subsystems of this combined quantum system are well understood. The combined system constitutes an archetype of matter-light interaction.

which are introduced into the description in Sec. 2.7.

\section{$2.1 \quad$ Jaynes-Cummings model}

The Jaynes-Cummings model (45) describes the fundamental system of matter-light interaction: a two-level atom $(\{|e\rangle,|g\rangle\})$ with transition frequency $\omega_{a}$ coupled to a quantized mode with Fock states $\left\{|n\rangle, n \in \mathbb{N}_{0}\right\}$ of a cavity with resonance frequency $\omega_{c}$ (Fig. 2.1). The atom is fixed in position with constant coupling $g$ and the interaction with the environment is neglected.

The interaction Hamiltonian $H_{d p}=-\mathbf{d} \cdot \mathbf{E}$ describes the coupling of the atomic dipole $\mathbf{d}$ to the electric field $\mathbf{E}$ and is considered in dipole and rotating wave approximation. Assuming that the wavelength of the light is large compared to the extension of the atomic wave packet, the field is regarded constant over the extension of the atom. In the dipole approximation the field is replaced by the value at the position of the atom $\mathbf{E}(\mathbf{r}, t)$. For a quantized field the operator $\mathbf{E}$ is replaced by four products $\left(a^{\dagger} \sigma^{-}\right.$, h.c.,$a^{\dagger} \sigma^{+}$, h.c.) of photon creation and annihilation operators $\left(a^{\dagger}, a\right)$ and the pseudospin operators of the atom $\left(\sigma^{+}, \sigma^{-}\right)$. In the uncoupled subsystems the phases of these oper- 
ators develop according to the Heisenberg equations of motion with their characteristic optical frequencies $\omega_{c}$ and $\omega_{a}$. The first two products ( $a^{\dagger} \sigma^{-}$, h.c.) correspond to the exchange of an excitation between atom and field. They evolve with the much smaller difference frequency $\Delta_{a c}=\omega_{a}-\omega_{c} \ll\left(\omega_{a}, \omega_{c}\right)$ and are preserved in the rotating-wave approximation. The 'anti-resonant' terms $\left(a^{\dagger} \sigma^{+}\right.$, h.c. $)$rotate with the sum of the frequencies. Their contributions average out on timescales shorter than the characteristic propagation of the system given by the detunings $\left(\Delta_{i}, i \epsilon\{a, c\}\right)$ with respect to the exciting laser $\left(\Delta_{i}=\omega_{i}-\omega_{l}\right)$ and the coupling $g$ (and decay rates $\{\gamma, \kappa\}$ for the open system in Sec. 2.2p. In the optical regime this rotating-wave approximation is generally well fulfilled.

The resultant Jaynes-Cummings Hamiltonian reads:

$$
\begin{aligned}
H_{J C} & =H_{a}+H_{c}+H_{a c} \\
H_{a} & =\hbar \omega_{a} \sigma^{+} \sigma^{-} \\
H_{c} & =\hbar \omega_{c} a^{\dagger} a \\
H_{a c} & =\hbar g\left(a^{\dagger} \sigma^{-}+a \sigma^{+}\right) .
\end{aligned}
$$

The first two contributions describe the atom and the field mode, respectively. $\left\{\sigma^{+}, \sigma^{-}, \sigma_{z}\right\}$ are the pseudospin operators of the atom and $\left\{a^{\dagger}, a\right\}$ the creation and annihilation operators of a photon in the mode. These operators fulfill the pseudospin algebra and commutation relation of the harmonic oscillator, respectively: 2.6):

$$
\begin{aligned}
{\left[\sigma^{+}, \sigma^{-}\right] } & =\sigma_{z},\left[\sigma_{z}, \sigma^{ \pm}\right]= \pm 2 \sigma^{ \pm} . \\
{\left[a, a^{+}\right] } & =1 .
\end{aligned}
$$

The dipole interaction couples product states $\left(|i, n\rangle:=|i\rangle \otimes|n\rangle ; i \in\{e, g\}, n \in \mathbb{N}_{0}\right)$ only pairwise in the subspaces $\{|g, n\rangle,|e, n-1\rangle\}$ of a given number of excitations $n$. The new eigenstates $|n, \pm\rangle$ and eigenenergies $E_{n, \pm}$ for the coupled system are given by:

$$
\begin{aligned}
|n, \pm\rangle & =c_{n, \pm}\left(|g, n\rangle \pm \alpha_{n, \pm}|e, n-1\rangle\right) \\
E_{n, \pm} & =\hbar\left(n \omega_{c}+\frac{1}{2}\left(\Delta_{a c} \pm \Omega_{n}\right)\right) \\
\alpha_{n, \pm} & =\frac{1}{\bar{\Omega}_{n}}\left(-\Delta_{a c} \mp \Omega_{n}\right) \\
c_{n, \pm} & =\left(1+\alpha_{n, \pm}^{2}\right)^{-1 / 2} \\
\Omega_{n} & =\sqrt{\Delta_{a c}^{2}+\left(\bar{\Omega}_{n}\right)^{2}} \\
\bar{\Omega}_{n} & =2 g \sqrt{n} .
\end{aligned}
$$

where $\Delta_{a c}=\omega_{a}-\omega_{c}$ is the atom-cavity detuning and $\bar{\Omega}_{n}$ the $n$ photon Rabi frequency. The generalized Rabi frequency $\Omega_{n}$ corresponds to the full energy difference between the energy levels of the coupled system (Fig. 2.2). For the degenerate case $\Delta_{a c}=0$ the generalized Rabi frequency $\Omega_{n}$ reduces to the $n$ photon Rabi frequency $\bar{\Omega}_{n}$. The absolute value of the levelshift in this resonant case given by $\bar{\Omega}$ is proportional to the 


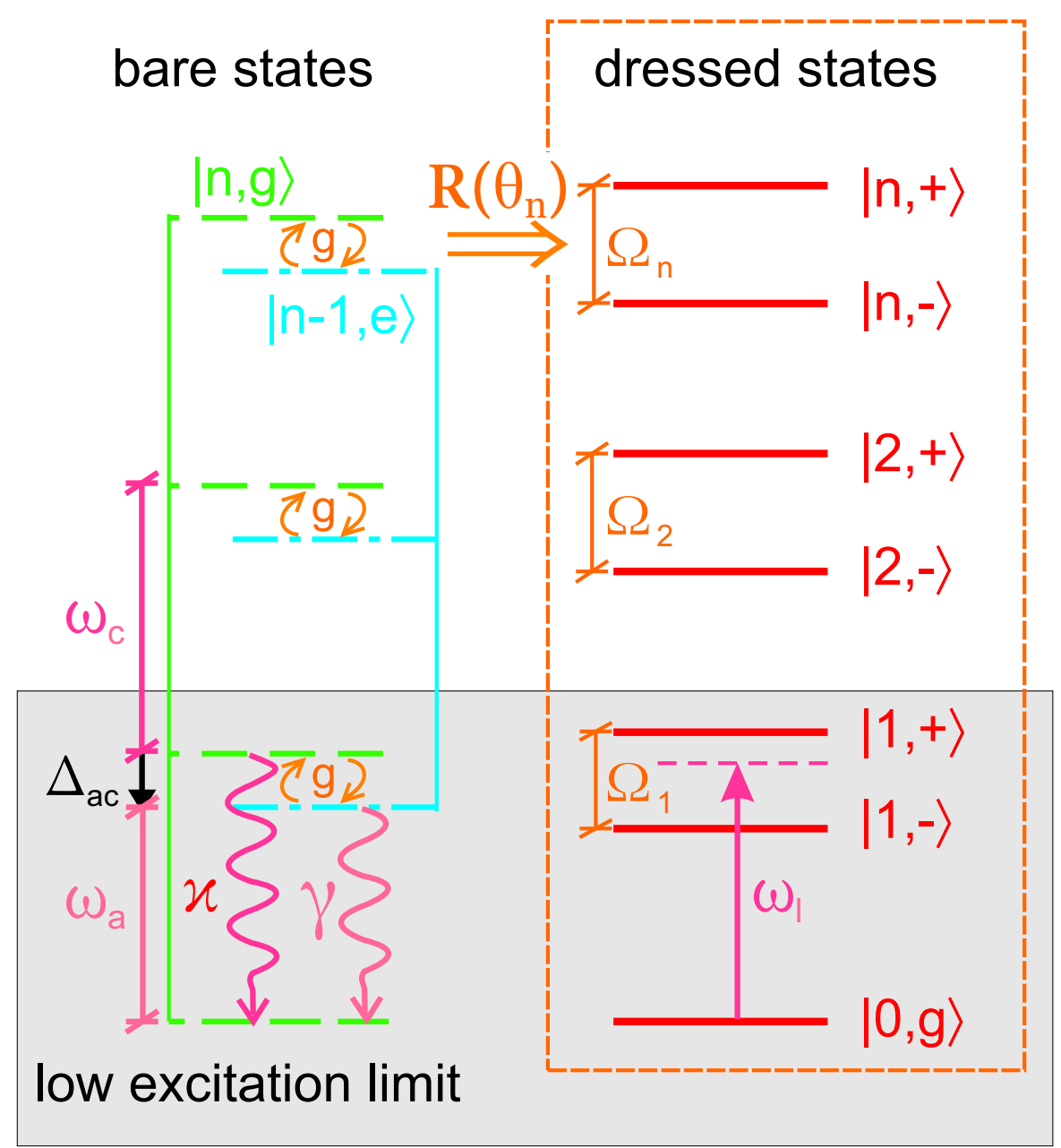

Figure 2.2: Normal modes of the Jaynes-Cummings model. For a bare frequency difference much smaller than the optical frequencies $\Delta_{a c}=\omega_{a}-\omega_{c} \ll\left(\omega_{a}, \omega_{c}\right)$ the product states form a ladder of pairs of states with equal number of excitations $n \in \mathbb{N}_{\geq 1}$. The distance between the center of two ladder steps $(n>1)$ is $\omega_{c}$. Note that the distance between the center of the first doublet and the ground state is instead given by the frequency mean $\left(\omega_{a}+\omega_{c}\right) / 2$.

The dipole interaction, in the rotating-wave approximation, couples product states of equal $n \in$ $\mathbb{N}_{\geq 1}$ with a bare frequency difference of $\Delta_{a c}=\omega_{a}-\omega_{c}$. These states split by the generalized Rabi frequency $\Omega_{n}$ into two 'dressed states' $\{|n,+\rangle,|n,-\rangle\}$ which are obtained by a rotation $\mathbf{R}\left(\Theta_{n}\right)$ in this subspace. Since these new eigenstates are generally not product states, the excitation and decays $(\gamma, \kappa)$ of the subsystems induce transitions between all combinations of states with $\Delta n= \pm 1$.

For low excitation one can restrict the discussion to the ground state $|g, 0\rangle$ and the lowest lying excited states $|1, \pm\rangle$. When scanning a probe beam across the normal modes one expects to see two resolved lines. In the degenerate case $\Delta_{a c} \equiv 0$ the splitting of the n-th doublet is given by $\bar{\Omega}_{n}=2 \sqrt{n} g$. 
coupling constant $g$ and scales with the square root of the number of excitations $n$. For $n=1$ this corresponds to the interaction of an excited atom with the vacuum field - the splitting by $2 g$ is therefore called 'vacuum-Rabi splitting'.

The new eigenstates of the coupled system of a two-level atom and cavity mode are called 'dressed states' (33). They arise from a rotation of the product states by an angle $\Theta_{n}$ in the two dimensional subspaces of equal number of excitation:

$$
\begin{aligned}
\left(\begin{array}{c}
|n,+\rangle \\
|n,-\rangle
\end{array}\right) & =\mathbf{R}\left(\Theta_{n}\right)\left(\begin{array}{c}
|g, n\rangle \\
|e, n-1\rangle
\end{array}\right) \\
\sin \left(\Theta_{n}\right) & =\frac{\bar{\Omega}_{n}}{\sqrt{\left(\Omega_{n}-\Delta_{a c}\right)^{2}+\left(\bar{\Omega}_{n}\right)^{2}}} .
\end{aligned}
$$

The rotary matrix $\mathbf{R}\left(\Theta_{n}\right)$ connects quantities (operators, probability amplitudes) in the bare state and in the dressed state basis. The progression of the coupled states with respect to the detuning $\Delta_{a c}$ corresponds to an 'anticrossing' (see Fig. 2.3). The smallest distance $\left(\Omega_{n}=\bar{\Omega}_{n}\right)$ between the dressed states is reached for the degenerate case. In this case the coupled states $|n, \pm\rangle$ have equal contributions from the bare states $\left(\Theta_{n}=45^{\circ}\right)$.

For $\Delta_{a c} \neq 0$ one of the normal modes is closer to the bare cavity (atom) state. The contribution of the cavity (atom) to this dressed state is larger than the one of the further detuned atom (cavity). This normal mode is hence called 'cavity like' ('atom like'). For large detuning $\left|\Delta_{a c}\right| / g \gg 1$, the dressed states coincide with the bare states apart from a small shift in energy and admixture of the other product state:

$$
\begin{aligned}
& |+\rangle \approx|g, n\rangle+1 / 2 \frac{\bar{\Omega}}{\Delta_{a c}}|e, n-1\rangle, E_{+} \approx \hbar\left(\omega_{c}+\frac{\bar{\Omega}^{2}}{4 \Delta_{a c}}\right) \\
& |-\rangle \approx 1 / 2 \frac{\bar{\Omega}}{\Delta_{a c}}|g, n\rangle-|e, n-1\rangle, E_{-} \approx \hbar\left(\omega_{a}-\frac{\bar{\Omega}^{2}}{4 \Delta_{a c}}\right) \text { (i.) }
\end{aligned}
$$

The state $|n,+\rangle$ is defined to be the state of higher energy. In the limit of large detuning $\omega_{c} \ll \omega_{a}$ and $\omega_{c} \gg \omega_{a}$, it approaches the bare state of the atom $|e, n-1\rangle$ and mode $|g, n\rangle$, respectively.

A weak probe beam exciting the system via the cavity drives transitions by adding an excitation to the cavity. It can be described by a time-dependent interaction Hamiltonian

$$
H_{s}(t)=-\hbar \eta\left(a^{\dagger} e^{-i \omega_{l} t}+\text { h.c. }\right) \text {. }
$$

The strength of the coherent excitation is given by the Rabi frequency $2 \eta$, which can be chosen to be real. In the theory of the open system in Sec. $2.2(\eta / \kappa)^{2}$ will turn out to be the steady state on resonance expectation value of the photon number in the empty cavity field. The perturbation $H_{s}(t)$ only drives transitions that add an excitation to the

\footnotetext{
${ }^{(i .)}$ For $\Delta_{a c}>0$ the mapping of the left hand sides to the normal modes $| \pm\rangle$ have to be interchanged to satisfy $E_{+}>E_{-}$. For low excitation, considering the ground state and the first excited pair only $n=1$, the Rabi frequency is given by $\bar{\Omega}=2 g$
} 


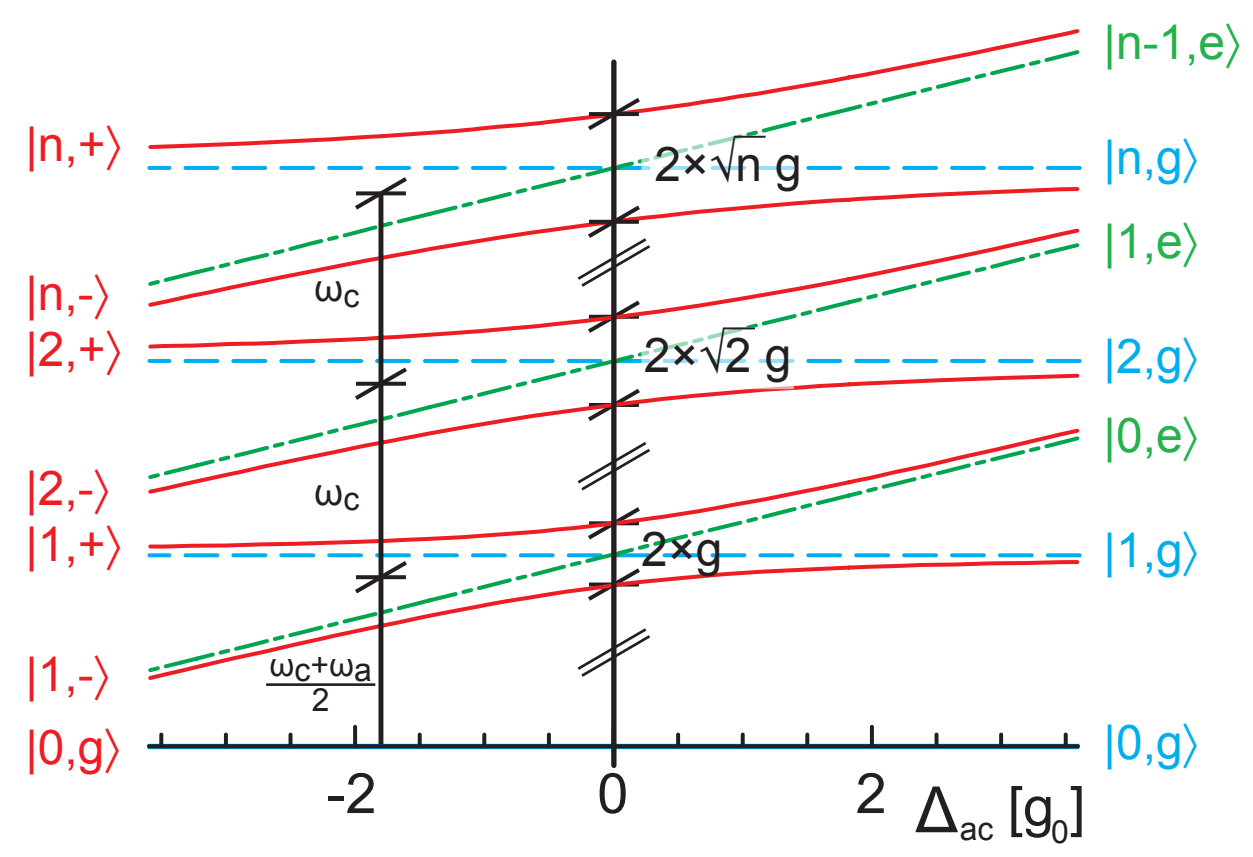

Figure 2.3: The normal modes versus the atom-cavity detuning $\Delta_{a c}$ : For large detuning the normal modes converge to the uncoupled product states of atom and mode. Due to the coupling the normal modes show an avoided crossing. In the degenerate case $\left(\Delta_{a c}=0\right)$ the normal modes consist of equal contributions of the bare states. The labels on the left side refer to the limiting product states (green,blue) for large detuning, while the right ones generally label the dressed states of the coupled system (red).

cavity mode. Restricting the discussion ground and first excited states (ii.), the strength of the absorption lines to the first coupled states at $\omega_{l}= \pm \sqrt{\Delta_{a c}^{2}+4 g^{2}}$ are given by the modulus square of the transition matrix elements:

$$
\begin{aligned}
\left\langle 0\left|H_{s}(t)\right|+\right\rangle & =-\hbar \eta e^{i \omega_{l} t} \cos (\Theta) \\
\left\langle 0\left|H_{s}(t)\right|-\right\rangle & =-\hbar \eta e^{i \omega_{l} t} \sin (\Theta) \\
\text { with } \tan (2 \Theta) & =-\frac{2 g}{\Delta}
\end{aligned}
$$

The excitation of the two normal modes $2.19,2.20)$, hence, differ in the detuned case. In the limit of large detuning $\left|\Delta_{a c} / g\right| \gg 1$ the spectrum will by dominated by the cavity-like dressed state, i.e. a resonance slightly shifted with respect to the bare cavity.

The Jaynes-Cummings Model can be extended to the Tavis-Cummings Model (107) to account for $N$ identical atoms with given couplings $g_{i}$ to a common mode. The many atoms turn out to behave like a single 'effective' atom with coupling $g_{\text {eff }}=$ $\sqrt{\sum_{i} g_{i}^{2}}$. The reason is that there is a single superposition of the atomic states $(|e g g . . g\rangle$

(ii.) At this point (until Chap. 8) we will restrict the discussion to the ground state and the lowest lying doublet: $|0\rangle \equiv|g, 0\rangle,| \pm\rangle \equiv|1, \pm\rangle\left(\Theta:=\Theta_{0}\right)$ 


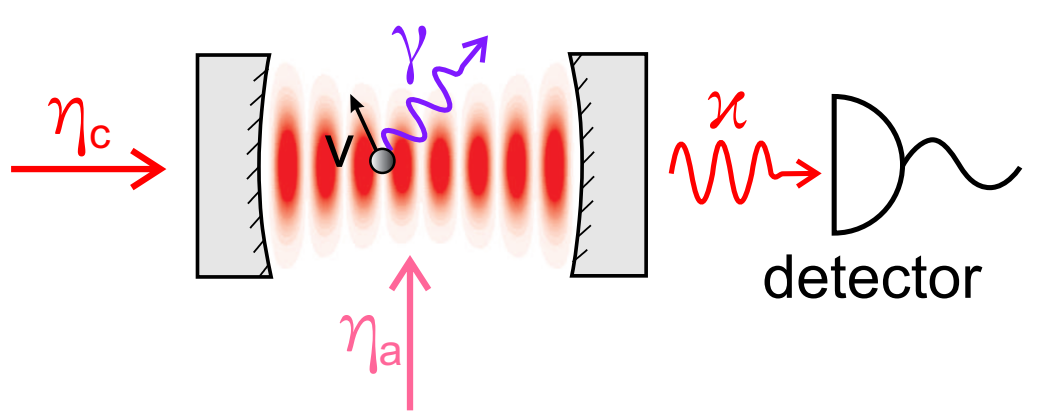

Figure 2.4: Atom-cavity system with losses. Both the atom and the cavity mode individually couple to the environment resulting in losses described by the cavity field decay rate $\kappa$ and decay rate of the atomic dipole $\gamma$. To compensate for the emitted energy, the system is pumped with probe fields via the cavity $\eta_{c}$ and/or the atom $\eta_{a}$. The retarded adoption of the system to variations of the coupling leads to strong velocity-dependent forces that can be both heating and cooling.

and permutations) that couples maximally to the mode while $N-1$ superpositions are uncoupled.

A corresponding generalization to many degenerate modes also leads to an 'effective' mode. It is given by the one superposition of the modes that maximally couples to the atom $\psi=\left(\sum_{i} g_{i} \psi_{i}\right) /\left(\sqrt{\sum_{i} g_{i}^{2}}\right)$. Since the transverse mode structure depends on the location of the atom it can be used to gain position information. This is the basis for the atomic kaleidoscope presented in Chap. 9.

\subsection{Open quantum system}

In the experiment the coupling to the environment leads to losses which cannot be neglected. The spontaneous emission of the atom and the transmission of the cavity mirrors are irreversible loss processes and lead to damping. The directed transmission via the cavity mirrors is well suited to continuously observe the system. To replenish the emitted energy, the system is pumped via the cavity $\left(\eta_{c} \neq 0\right)$ and/or the atom with Rabi frequencies $\left(\eta_{a} \neq 0\right)$, where $2 \eta_{i}$ is the Rabi frequency (Fig. 2.4).

The discussion of the interaction with the environment in this section follows the Schrödinger picture and leads to the master equation. The environment is introduced by coupling the system $\mathrm{S}$ to a bath of harmonic oscillators or reservoir $\mathrm{R}$. The total Hamiltonian is the sum of Hamiltonians describing system, reservoir, their interaction and the pump

$$
H_{\mathrm{tot}}=H_{S}+H_{R}+H_{S R}+H_{P}
$$

The Hilbert space is the by the tensor product $\mathcal{H}=\mathcal{H}_{S} \otimes \mathcal{H}_{R}$. The reservoir can be modelled as a bath of harmonic oscillators, representing the modes of the light field 
outside the cavity, described by the Hamiltonian

$$
H_{R}=\sum_{k} \omega_{k}\left(b_{k}^{\dagger} b_{k}+\frac{1}{2}\right),
$$

where $b_{k}^{\dagger}, b_{k}$ are the photon creation and annihilation operator for photons in mode $k$ and $\omega_{k}$ is the frequency of the oscillator. In case of the atom-cavity system $\left(H_{S}=H_{J C}\right)$, it is assumed that the reservoir is weakly coupled to the atom and the cavity, with coupling constants $g_{\mathrm{a}, k}$ and $g_{\mathrm{c}, k}$, respectively. The system reservoir interaction is given by:

$$
H_{S R}=\sum_{k} g_{\mathrm{c}, k}\left(b_{k}^{\dagger} a+a^{\dagger} b_{k}\right)+\sum_{k} g_{\mathrm{a}, k}\left(b_{k}^{\dagger} \sigma^{-}+\sigma^{+} b_{k}\right) .
$$

The exchange of energy between system and reservoir is thus assumed to consist of the simultaneous creation of one quantum of excitation of the system and annihilation of a quantum in the $k$-th mode of the bath or vice versa.

The time evolution of the total system is formally given by the von-Neumann equation for the density matrix $\rho_{S R}$

$$
\dot{\rho}_{t o t}=-i\left[H_{t o t}, \rho_{t o t}\right],
$$

which in general cannot be solved analytically. Since the interest is in the time evolution of the system only, the reduced density operator of the system is obtained by tracing over the reservoir, yielding

$$
\rho_{S}=\operatorname{Tr}_{R}\left(\rho_{t o t}\right) .
$$

It is assumed that the reservoir has a large bandwidth such that correlations decay much faster than the timescale of the interaction with the system. The state of the reservoir does not depend on the state of the system at an earlier time, i.e. it has no memory of the dynamics of the system. Therefore, the influence of the environment on the system only depends on the current state of the system (Markov approximation). At room temperature the relevant modes of the reservoir at optical frequencies are not excited (iii.) Tracing out the reservoir in thevacuum state, the master equation for the JaynesCummings system, which is weakly coupled to the environment, reduces in Born-Markov approximations to (108):

$$
\begin{aligned}
\dot{\rho} & =\mathcal{L} \rho=-\frac{i}{\hbar}\left[H_{J C}+H_{P}, \rho\right] \\
& +\kappa\left(2 a \rho a^{\dagger}-a^{\dagger} a \rho-\rho a^{\dagger} a\right) \\
& +\gamma\left(2 \sigma^{-} \rho \sigma^{+}-\sigma^{+} \sigma^{-} \rho-\rho \sigma^{+} \sigma^{-}\right) .
\end{aligned}
$$

Here, $\mathcal{L}$ is called the Liouville super operator. The interaction with the environment is represented by the decay rates $\kappa$ and $\gamma$ for the field of the cavity mode and the atom

(iii.) For a finite temperature $T$ only the mean occupation of the modes $\bar{n}\left(\omega_{k}, T\right)=\frac{e^{-\hbar \omega_{k} / k_{B} T}}{1-e^{-\hbar \omega_{k} / k_{B} T}}$ would enter as a factor $(\bar{n}+1)$ to $\gamma$ and $\kappa$, as well as, to the conjugate Lindblad forms in 2.27) with a factor of $\bar{n}$. However, $\hbar \omega_{k} / k_{B} T \gg 1$ and therefore $n\left(\omega_{k}, T\right) \approx n\left(\omega_{k}, 0\right)=0$ 
polarization, respectively. Even though the mirrors change the mode density along the axial direction, the spontaneous emission rate is assumed not to be influenced by the cavity surrounding the atom. This is a reasonable approximation since the cavity normally covers only a small fraction of the solid angle.

\subsubsection{Quantum regression theorem}

The equation of motion for the expectation value of any system operator $O$ can be calculated from the density operator $\rho$ :

$$
\langle\dot{O}\rangle=\operatorname{Tr}_{S}\{O \dot{\rho}\}=\operatorname{Tr}_{S}\{O \mathcal{L}(\rho)\} .
$$

The forces and the diffusion in the system are given by two-time expectation values $\left\langle O_{1}(t) O_{2}(t+\tau)\right\rangle$ of two system operators $O_{1}, O_{2}$. These can be evaluated using the quantum regression theorem (108):

$$
\begin{aligned}
& \left\langle O_{1}(t) O_{2}(t+\tau)\right\rangle=\operatorname{Tr}_{S}\left\{O_{2}(0) e^{\mathcal{L} \tau}\left[\rho(t) O_{1}(0)\right]\right\} \\
& \left\langle O_{1}(t+\tau) O_{2}(t)\right\rangle=\operatorname{Tr}_{S}\left\{O_{1}(0) e^{\mathcal{L} \tau}\left[O_{2}(0) \rho(t)\right]\right\} .
\end{aligned}
$$

In this formal solution the time propagation is given by the Liouville super operator $\mathcal{L}$. It can be reduced to a form more appropriate for evaluation by introducing a complete set of system operators combined to a vector $\mathbf{A}$. If the time evolution of $\mathbf{A}$ is a set of linear equations given by the evolution matrix $\mathbf{M}$ :

$$
\langle\dot{\mathbf{A}}\rangle=\mathbf{M}\langle\mathbf{A}\rangle,
$$

the two-time averages with an arbitrary system operator $O$ can be calculated as $(\tau \geq 0)$ :

$$
\begin{aligned}
\frac{\mathrm{d}}{\mathrm{d} \tau}\left\langle O_{1}(t) \mathbf{A}(t+\tau)\right\rangle & =\mathbf{M}\left\langle O_{1}(t) \mathbf{A}(t+\tau)\right\rangle \\
\frac{\mathrm{d}}{\mathrm{d} \tau}\left\langle\mathbf{A}(t+\tau) O_{2}(t)\right\rangle & =\mathbf{M}\left\langle\mathbf{A}(t+\tau) O_{2}(t)\right\rangle .
\end{aligned}
$$

According to this equation, the equation of motion for the correlation functions (two-time averages) can be directly deduced from the equation of motion for the system operators (one-time averages).

For the atom-cavity system linear equations of motion can be obtained in the limit of low excitation or in the harmonic limit. A full set of system operators is then given by the photon annihilation operator $a$ and the Pauli pseudo-spin operator $\sigma^{-}$. Since the normal-ordered operator products factorize, the evaluation generates extra terms when reordering the products only.

\subsection{Atomic motion and light force}

To include the light forces acting on the atom, the motional degrees of freedom have to be included. The coupling is proportional to the field at the atomic position. Hence, its 
spatial dependence $g(\mathbf{r})=g_{0} \phi(\mathbf{r})$ is given by the mode function $\phi(\mathbf{r})$ normalized to 1 at an antinode where the maximum coupling $g_{0}$ is reached (for more details see 9.1). The force operator can be derived from the full position dependent Hamiltonian. However, because in the current system the DeBroglie wavelength of the atom is small compared to the wavelength, it is sufficient to ultimately treat the motional degrees semi-classically.

\subsubsection{Force operator}

Introducing the spatial dependence of the parameters and the kinetic energy $\mathbf{P}^{2} / 2 m$ of the atom with mass $m$, the full position-dependent Hamiltonian is given by:

$$
\begin{aligned}
H(\mathbf{r})=\frac{\mathbf{P}^{2}}{2 m}+\hbar \omega_{a} \sigma^{+} \sigma^{-}+\hbar \omega_{c} a^{+} a+\hbar g \phi(\mathbf{r})\left(a^{+} \sigma^{-}+\sigma^{+} a\right) & \\
& +H_{P}+H_{R}+H_{S R} .
\end{aligned}
$$

The force operator is defined as the time derivative of the momentum and given by the Heisenberg equation of motion for the atomic momentum operator:

$$
\mathbf{F}=\dot{\mathbf{P}}=\frac{i}{\hbar}[H, \mathbf{P}]
$$

Using eqn. (2.34), the expectation value of the force is given by

$$
\langle\mathbf{F}\rangle=-\hbar g_{0}(\nabla \phi(\mathbf{r}))\left\langle a^{+} \sigma^{-}+\sigma^{+} a\right\rangle .
$$

It can be evaluated once the steady-state expectation values of the operators involved are known, e.g. in the low excitation limit (Sec. 2.4). Other terms contribute only to the fluctuations of the force, leading to a spreading of the atomic wavepacket. The first moment of this spreading is the momentum diffusion coefficient discussed in Sec. 2.5.

\subsection{Low-excitation limit}

The equations of motion for the expectation values of the system operators $\sigma^{-}$and $a$ can be calculated using 2.25) and the master equation 2.27). In the rotating-wave approximation with respect to the laser frequency $\omega_{p}$, the following detunings are introduced (109):

$$
\begin{array}{ll}
\Delta_{a}:=\omega_{p}-\omega_{a} & \tilde{\Delta}_{\mathrm{a}}:=\Delta_{a}+i \gamma \\
\Delta_{c}:=\omega_{p}-\omega_{c} & \tilde{\Delta}_{\mathrm{c}}:=\Delta_{c}+i \kappa .
\end{array}
$$

The equations of motion for the expectation values of the system operators $\langle a\rangle,\left\langle\sigma^{-}\right\rangle$ and $\left\langle\sigma_{z}\right\rangle$ can be written as

$$
\begin{aligned}
\langle\dot{a}\rangle & =i \tilde{\Delta}_{\mathrm{c}}\langle a\rangle-i g(\mathbf{r})\left\langle\sigma^{-}\right\rangle+\eta_{\mathrm{c}} \\
\left\langle\dot{\sigma}^{-}\right\rangle & =i g(\mathbf{r})\left\langle\sigma_{z} a\right\rangle+i \tilde{\Delta}_{\mathrm{a}}\left\langle\sigma^{-}\right\rangle+\eta_{\mathrm{a}} \\
\left\langle\dot{\sigma}_{z}\right\rangle & =i g(\mathbf{r})\left\langle a^{+} \sigma^{-}-\sigma^{+} a\right\rangle-2 \gamma\left\langle\sigma^{+} \sigma^{-}\right\rangle .
\end{aligned}
$$


An analytical solution for this system of non-linear differential equations leads to optical bistability (110). In the limit of weak atomic excitation, the algebra of the pseudo-spin operators (2.5) can be approximated by the algebra of the harmonic oscillator (2.6), i.e. $\left[\sigma^{-}, \sigma^{+}\right]=1$. This corresponds to replacing $\left\langle\sigma_{z} a\right\rangle$ by $-\langle a\rangle$ in 2.40) and dropping (2.41). The linearized equation of motion can be written in matrix notation:

$$
\begin{gathered}
\langle\dot{\mathbf{Y}}\rangle=\mathbf{Z}\langle\mathbf{Y}\rangle+\mathbf{I} \\
\mathbf{Y}:=\left(\begin{array}{c}
a \\
\sigma^{-}
\end{array}\right), \mathbf{Z}:=\left(\begin{array}{cc}
i \tilde{\Delta}_{\mathrm{c}} & -i g \\
-i g & i \tilde{\Delta}_{\mathrm{a}}
\end{array}\right), \mathbf{I}:=\left(\begin{array}{l}
\eta_{\mathrm{c}} \\
\eta_{\mathrm{a}}
\end{array}\right) .
\end{gathered}
$$

By inverting (2.42) the steady state solution is obtained:

$$
\langle\mathbf{Y}\rangle=-\mathbf{Z}^{-1} \mathbf{I} .
$$

In the following the pump term will be restricted to pumping the cavity as implemented in the experiment, to avoid complication i.e. $\eta_{\mathrm{a}} \equiv 0$. The steady state solution is then given by

$$
\langle\mathbf{Y}\rangle_{0}=\left\langle\left(\begin{array}{c}
a \\
\sigma^{-}
\end{array}\right)\right\rangle_{0}=\frac{i \eta}{g^{2}-\tilde{\Delta}_{\mathrm{a}} \tilde{\Delta}_{\mathrm{c}}}\left(\begin{array}{c}
\tilde{\Delta}_{\mathrm{a}} \\
g
\end{array}\right)
$$

Since the expectation value of normal ordered products of operators factorise in the coupled oscillator model ((111), page 27), various expectation values for physical quantities can directly be calculated from $(2.45)$. The steady state expectation values for the intracavity photon number $\left\langle a^{+} a\right\rangle=\left\langle a^{+}\right\rangle\langle a\rangle$, the atomic excitation $\left\langle\sigma^{+} \sigma^{-}\right\rangle=\left\langle\sigma^{+}\right\rangle\left\langle\sigma^{-}\right\rangle$ and the dipole force are given by

$$
\begin{aligned}
\left\langle a^{+} a\right\rangle_{0} & =\eta^{2} \frac{\left|\tilde{\Delta}_{\mathrm{a}}\right|^{2}}{\left|g^{2}-\tilde{\Delta}_{\mathrm{a}} \tilde{\Delta}_{\mathrm{c}}\right|^{2}} \\
\left\langle\sigma^{+} \sigma^{-}\right\rangle_{0} & =\eta^{2} \frac{g^{2}}{\left|g^{2}-\tilde{\Delta}_{\mathrm{a}} \tilde{\Delta}_{\mathrm{c}}\right|^{2}}=\frac{g^{2}}{\Delta_{a}^{2}+\gamma^{2}}\left\langle a^{+} a\right\rangle_{0}
\end{aligned}
$$

The dipole force acting on an atom at rest is obtained from 2.36

$$
\begin{aligned}
\langle\mathbf{F}(\mathbf{r})\rangle_{0} & =-\hbar \eta^{2}(\nabla g(\mathbf{r})) \frac{2 \Delta_{a} g(\mathbf{r})}{\left|g^{2}-\tilde{\Delta}_{a} \tilde{\Delta}_{c}\right|^{2}} \\
& =-\hbar \frac{\Delta_{a}\left(\nabla g^{2}(\mathbf{r})\right)}{\Delta_{a}^{2}+\gamma^{2}}\left\langle a^{+} a\right\rangle_{0} .
\end{aligned}
$$

The conservative dipole force can be written as the derivative of a potential $\langle\mathbf{F}(\mathbf{r})\rangle_{0}=$ $-\nabla V(\mathbf{r})$ :

$$
\begin{aligned}
V(\mathbf{r}) & =-\frac{\hbar \eta^{2} \Delta_{a}}{\mathcal{I} m(\mathcal{A})} \arctan \left(\frac{\mathcal{R} e(\mathcal{A})}{\mathcal{I} m(\mathcal{A})}\right), \operatorname{Im}(\mathcal{A}) \neq 0 \\
V(\mathbf{r}) & =-\frac{2 \hbar \eta^{2} \Delta_{a}}{\mathcal{R} e(\mathcal{A})^{3}}, \mathcal{I} m(\mathcal{A})=0 \\
\mathcal{A} & :=g^{2}-\tilde{\Delta}_{a} \tilde{\Delta}_{\mathrm{c}} .
\end{aligned}
$$


Depending on the sign of the laser-atom detuning $\Delta_{\mathrm{a}}$, the atom is attracted to regions of high (low) intensity for $\Delta_{\mathrm{a}}<0\left(\Delta_{\mathrm{a}}>0\right)$, i.e. red (blue) detuned light field.

\subsection{Momentum diffusion}

The mean value of the dipole force 2.48 is driving the center of mass motion of the atomic wavepacket. In addition, the fluctuation of this force leads to a spreading of the atomic momentum distribution quantified by the variance of the momentum

$$
(\Delta P)^{2}(t)=\left\langle[\mathbf{P}(t)-\langle\mathbf{P}(t)\rangle]^{2}\right\rangle .
$$

In linear order the spreading is characterized by the diffusion tensor. Restricting the discussion to the one-dimensional case, it is given by the diffusion coefficient $D$, defined as

$$
2 D=\frac{\mathrm{d}}{\mathrm{d} t}(\Delta P)^{2}(t)
$$

The diffusion coefficient can be rewritten in terms of two-time correlations of the force operator starting with the formal solution using eqn. (2.53):

$$
\begin{aligned}
\frac{\mathrm{d}}{\mathrm{d} t}\left\langle[\mathbf{P}(t)-\langle\mathbf{P}(t)\rangle]^{2}\right\rangle & =\frac{\mathrm{d}}{\mathrm{d} t}\left\langle\mathbf{P}^{2}\right\rangle-2\langle\mathbf{P}\rangle \frac{\mathrm{d}}{\mathrm{d} t}\langle\mathbf{P}\rangle \\
& =\langle\mathbf{F} \cdot \mathbf{P}+\mathbf{P} \cdot \mathbf{F}\rangle-2\langle\mathbf{P}\rangle \cdot\langle\mathbf{F}\rangle .
\end{aligned}
$$

Inserting the formal solution

$$
\mathbf{P}(t)=\int_{0}^{\infty} \mathrm{d} \tau \mathbf{F}(t-\tau)+\mathbf{P}(0)
$$

for the atomic momentum and (2.55) into eqn. (2.54) yields

$$
\begin{aligned}
2 D & =2 \mathcal{R} e \int_{0}^{\infty} \mathrm{d} \tau(\langle\mathbf{F}(t) \cdot \mathbf{F}(t-\tau)\rangle-\langle\mathbf{F}(t)\rangle \cdot\langle\mathbf{F}(t-\tau)\rangle) \\
& =2 \mathcal{R} e \int_{0}^{\infty} \mathrm{d} \tau\langle\delta \mathbf{F}(t) \cdot \delta \mathbf{F}(t-\tau)\rangle .
\end{aligned}
$$

The eqn. 2.57) can be used to calculate the momentum diffusion coefficient. For a fixed atom and weak atomic excitation, the momentum diffusion coefficient was calculated by Hechenblaikner et al. (85). The result reads

$$
\begin{aligned}
D & =D_{s e}+D_{d p} \\
D_{s e} & =\hbar^{2} k^{2} \gamma\left\langle\sigma^{+} \sigma^{-}\right\rangle=\hbar^{2} k^{2} \gamma \frac{\eta^{2} g^{2}}{\left|g^{2}-\tilde{\Delta}_{\mathrm{a}} \tilde{\Delta}_{\mathrm{c}}\right|^{2}} \\
D_{d p} & =\hbar^{2}(\nabla g)^{2} \frac{\eta^{2} \gamma}{\left|g^{2}-\tilde{\Delta}_{\mathrm{a}} \tilde{\Delta}_{\mathrm{c}}\right|^{2}}\left(1+\frac{4 \Delta_{a} g^{2}}{\gamma} \frac{\Delta_{c} \gamma+\Delta_{a} \kappa}{\left|g^{2}-\tilde{\Delta}_{\mathrm{a}} \tilde{\Delta}_{\mathrm{c}}\right|^{2}}\right) \\
& =D_{\text {free }}\left(1+\frac{4 \Delta_{a} g^{2}}{\gamma} \frac{\Delta_{c} \gamma+\Delta_{a} \kappa}{\left|g^{2}-\tilde{\Delta}_{\mathrm{a}} \tilde{\Delta}_{\mathrm{c}}\right|^{2}}\right)
\end{aligned}
$$


Here, the momentum diffusion coefficient $D_{s e}$ is generated by spontaneous emission of photons from the atom, while $D_{d p}$ is due to fluctuations of the dipole force.

$$
D_{\text {free }}=\hbar^{2}(\nabla g)^{2} \frac{\eta^{2} \gamma}{\left|g^{2}-\tilde{\Delta}_{\mathrm{a}} \tilde{\Delta}_{\mathrm{c}}\right|^{2}}
$$

is the diffusion coefficient for an atom in a free-space standing wave light field (112). Equation 2.60) shows that the diffusion in a cavity can be much stronger than in freespace. $D_{d p}$ can be rewritten in the invariant form of two symmetric terms (86):

$$
D_{d p}=\left|\hbar \nabla\left\langle\sigma^{-}\right\rangle\right|^{2} \gamma+|\hbar \nabla\langle a\rangle|^{2} \kappa
$$

The first term can be interpreted as a fluctuating dipole coupled to a classical field, as in free space. The second term corresponds to a classical dipole coupled to a fluctuating field. Note that the effect of the mutual backaction of the subsystems is included in the evaluation of the steady-state expectation values. Their evaluation lead to the known complicated expression obtained for the different configurations.

\subsection{Velocity-dependent forces}

A point-like atom moving within the cavity mode experiences a locally varying coupling. The steady state of the atom-cavity system depends on the coupling strength, but its value is not established instantaneously. The system can only adopt to the new steady state on the timescale of the atomic and cavity decay. Hence, in the case of a moving atom, the system does not reach the steady state corresponding to the actual atomic position but lags behind it. To describe this effect, the dipole force for a resting atom (2.48) must be extended by a velocity-dependent correction.

For an atom which moves only a small fraction of a wavelength during the relaxation time of the system, $\mathbf{k} \cdot \mathbf{v} \ll(\gamma, \kappa)$, the velocity-dependent force can be approximated by a term linear in the velocity. This can be derived by expanding the density operator of the system in powers of the atomic velocity: $\rho=\rho_{0}+\rho_{1}+\cdots$. To calculate the expectation values up to the first order of the atomic velocity, the total derivative of the density matrix can be split into partial derivatives (hydrodynamic derivative)

$$
\frac{\mathrm{d}}{\mathrm{d} t} \rho=\frac{\partial}{\partial t} \rho+\mathbf{v} \cdot \nabla \rho
$$

In the same way the total derivative of $\langle\mathbf{Y}\rangle$ can be written as

$$
\langle\dot{\mathbf{Y}}\rangle=\left(\frac{\partial}{\partial t}+\mathbf{v} \cdot \nabla\right)\langle\mathbf{Y}\rangle
$$

It is assumed that there is no explicit (external) time-dependence of the Hamiltonian and therefore $\frac{\partial}{\partial t}\langle\mathbf{Y}\rangle=0$. Using equation (2.42) and considering only the first order in the atomic velocity, the first-order correction for the expectation values is

$$
\langle\mathbf{Y}\rangle_{1}=\mathbf{Z}^{-1} \mathbf{v} \cdot \nabla\langle\mathbf{Y}\rangle_{0}
$$


where $\langle\mathbf{Y}\rangle_{0}$ is the steady-state solution for a fixed atom. This result can be used to calculate the expectation value of the force operator in first order of the velocity $\mathbf{v}$,

$$
\begin{aligned}
\langle\mathbf{F}\rangle_{1} & =-\hbar(\nabla g)\left(\left\langle a^{+}\right\rangle_{0}\left\langle\sigma^{-}\right\rangle_{1}+\left\langle a^{+}\right\rangle_{1}\left\langle\sigma^{-}\right\rangle_{0}\right)+\text { c.c. } \\
& =:-\beta \mathbf{v}
\end{aligned}
$$

The coefficient $\beta$ is called friction coefficient. The analytic result for this velocitydependent force evaluated for the given setting is lengthy and can be found in (85).

\subsection{Intracavity dipole trap}

As discussed above, heating due to dipole force fluctuations induced by the probe can be particularly strong in a cavity. It is therefore favorable to separate the trapping mechanism from the near resonant cavity QED mode. An established tool to localize polarizable particles like atoms are far-detuned optical dipole traps (113), because the scattering rate decreases linearly with the detuning at fixed trap depth. In the cavity setting, far-detuned modes are well suited to localize the atom (82). The description of the dipole modes simplifies because the back action of the atom on the field can be neglected for very large detunings $\Delta_{\text {trap }} \gg g$ and large photon numbers $n_{\text {trap }} \gg 1$. Therefore, the far-detuned intracavity dipole trap can be included into the description by a position dependent atomic detuning, $\Delta_{\mathrm{a}}(\mathbf{r})=\Delta_{\mathrm{a}}-2 \Delta_{\mathrm{S}}(\mathbf{r})$, modified according to the Stark shift $\Delta_{\mathrm{S}}(\mathbf{r})$ introduced by the trapping field:

$$
\Delta_{\mathrm{S}}(\mathbf{r})=-\frac{g_{\text {trap }}^{2}\left|\psi_{\text {trap }}(\mathbf{r})\right|^{2} n_{\text {trap }}}{\Delta_{\text {trap }}} .
$$

Where the maximum coupling $g_{\text {trap }}$ is calculated for the detuned cavity mode $\psi_{\text {trap }}(\mathbf{r})$. The low excitation limit $(91 ;$; 85$)$ is extended by introducing $\Delta_{\mathrm{a}}(\mathbf{r})$ into the Hamiltonian to obtain analytical equations including the trap.The steady-state expectation values are obtained by direct substitution of $\Delta_{\mathrm{a}}(\mathbf{r})$. In contrast, all quantities containing derivatives are generally extended by extra terms involving gradients of the position dependent atomic detuning, as well as mixed terms with the gradient of the coupling. The full dipole force is hence given by the contributions of the near-resonant mode and all dipole fields which can be described by a combined position-dependent Stark shift $\Delta_{\mathrm{S}}(\mathbf{r})$. In three dimensions, this results in tensor quantities for the diffusion and velocity-dependent force. The expressions for a given pump geometry can be found elsewhere (87), where the spatial dependence of the Stark shift is given by the detunings and geometry of all dipole modes.

In fact, any spatial parameter variation leads to extra terms. Analogous to the variation of the coupling discussed above, this generally leads to velocity-dependent forces which can be heating or cooling. Because strong forces are induced by pronounced gradients the velocity-dependent force due to the variation of the coupling mostly acts in the axial direction. Three-dimensional cavity cooling can be implemented by additional spatial parameter variations in the directions orthogonal to the cavity axis. For example, 
pumping the system from the side with varying amplitude or polarization, or modulating the atom-cavity detuning by the AC-Stark shift of additional orthogonal dipole fields.

If instead of the cavity the system is pumped via the atom only $\left(\eta_{\mathrm{a}} \neq 0, \eta_{\mathrm{c}} \equiv 0\right)$ a symmetric picture is obtained with the roles of atom and cavity interchanged. Inevitably, the gradients of the coupling still result in axial cavity cooling. Moreover, including a spatial structure in $\eta_{\mathrm{a}}(\mathbf{r})$ will lead to velocity-dependent forces in the direction of the side pump, i.e. $\nabla \eta_{\mathrm{a}}$. As discussed in detail in sections 4.1 .2 and 7.9 there is a common parameter regime which allows for three-dimensional cavity cooling. A major motivation for the development of the blue trap presented in the next Chap. 3 is the possibility to trap atoms in this parameter regime as presented in Sec. 7.9. 



\section{Chapter 3}

\section{The idea of the blue intracavity dipole trap}

A strongly-coupled atom-mode system is prepared by guiding and trapping single atoms in the region of strong coupling using intracavity modes. The atom can be stored in the near resonant red-detuned probe field $(101 ;$;4). However, the trap depth in the low excitation limit is limited to $<1 \mathrm{mK}$, and therefore, trapping times are restricted to a few times the transit time or inverse radial trap frequency. To improve localization of the atom additional far-detuned light fields are used for trapping. At a given trap depth, the excitation of the atom by the trapping field, and hence heating due to spontaneous emission, is suppressed inversely with the detuning from the atomic transition frequency.

For small Fabry-Perot cavities that allow for strong-coupling, optical access from the side is limited; therefore, intracavity dipole traps are preferred (Sec. 3.1). Since an atom is attracted to the intensity maxima, the antinodes of a single far red-detuned mode provides a one-dimensional array of traps (Sec. 3.2). In the red trap the energy levels are shifted by the AC-Stark effect with respect to the free atom. A repellant blue-detuned standing-wave light field only provides one-dimensional confinement to the nodal planes. Additional higher-order modes are necessary for complete three-dimensional confinement (Sec. 3.3). In the blue trap the atom is completely surrounded by blue light and stored close to the dark center where the free-space energy levels are preserved. A number of advantages result from this characteristic of the blue-detuned intracavity dipole trap. The discussion is extended in Chap. 4, where two experimentally relevant parameter regimes are introduced.

\subsection{Intracavity dipole traps}

An intracavity dipole trap is composed out of a set of far-detuned eigenmodes of the high-finesse cavity. These modes have a well defined spatial structure and orientation. Because the intracavity field is enhanced by a factor in the order of the finesse, strong fields can be built up by coupling only a weak laser beam to the mode. In addition, the high-finesse cavity provides single-atom detection capability in the strong-coupling 
regime (100; 98,99$)$, which is a prerequisite to prepare a single-atom cavity-system.

The preparation of a strongly coupled single atom-mode system is a multi-step process: 1. The atom has to be guided into regions of strong coupling. 2. It is detected via its strong influence on the cavity field transmission. 3. Upon detection it is trapped by switching the conservative dipole trap. 4 . To improve the localization, the atom is cooled to compensate for any energy increase during loading and the presence of heating.

\subsection{The red trap}

A red detuned dipole trap can be realized by a single standing-wave cavity mode. The atoms are attracted to the intensity maxima at the antinodes which define local trap centers. When the mode is an even number of free-spectral ranges (FSR) detuned from the near-resonant cavity mode, the antinodes of the probe mode are aligned with the trap centers half-way between the mirrors, see Fig. 3.1. Since the atom is trapped at maximum field intensity, its transition frequency is modified by the differential Stark shift of the ground and excited state. For a two-state atom the Stark shift equals twice the trap depth because ground and excited state repel from each other by the same level shift. In a real atom, higher lying states have to be included and lead to a combined shift that strongly depends on the detuning in the proximity of other resonances. Note that for experiments on the $D_{2}$-line of ${ }^{85} \mathrm{Rb}$ at $780.24 \mathrm{~nm}$ the detuning for the dipole trap is restricted to values smaller than half way to the $D_{1}$-line at $795 \mathrm{~nm}$, or much larger detunings, for all but the extreme hyperfine sublevel. In the experiment the probe and trap field are both circularly polarized and optically pump the atom into this extreme hyperfine sublevel providing a true 'two-level' system. Due to the individual level structure in Cesium, there is a special 'magic wavelength' for which the coupling to higher levels leads to an combined downward Starkshift on the excited state that matches the ground state shift (95). At this wavelength the atomic transition frequency is not altered by the trap. In ${ }^{85} \mathrm{Rb}$ a red-detuned magic wavelength exists in the far infrared at $1529 \mathrm{~nm}$ but is experimentally impractical. There is, on the other hand, a blue-detuned magic wavelength close to $776 \mathrm{~nm}$.

To load the red-detuned trap, slow atoms are guided by the potential of a weak dipole field. Since the atom 'rolls down' the potential dimple, at any time its energy is sufficient to reside anywhere in the mode. When an atom, strongly coupled to the near-resonant mode is detected, the conservative potential is switched to trap the atom. The coupling and hence, the radial position can be directly observed and qualified via the cavity transmission. In contrast, the axial motion is about one and a half orders of magnitude faster and cannot be resolved. Therefore, the position at the switching time along the standing wave is arbitrary. Atoms that are close to the node are not trapped by the switching. Generally, the average axial energy gain will be about half the potential depth. Still, when efficient cavity cooling in axial direction is present, close to unity trapping efficiencies are achieved, i.e. no extra losses on a short timescale are observed (88). In the far-detuned dipole trap the storage times are an order of magnitude larger than in the near-resonant field. In the dark trap, without probe light, the storage 


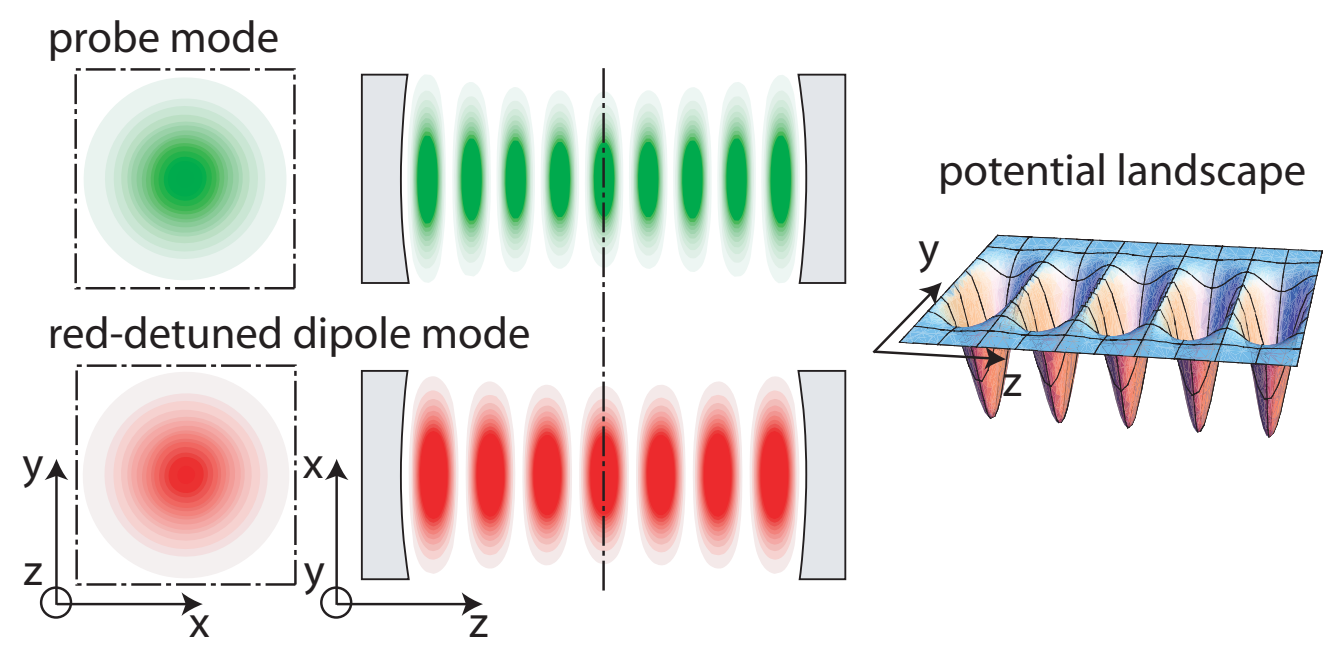

Figure 3.1: Red intracavity dipole trap. The basic red detuned intracavity dipole trap consists of a single $\mathrm{TEM}_{00}$ mode. It is an odd number of free-spectral ranges red detuned from the probe, such that the local trap centers given by the intensity maxima (red) overlap with the antinodes of the near-resonant probe field (green) at the cavity center. An atom is guided by a weak conservative dipole field which is switched upon the detection of a well-coupled atom. The potential landscape corresponds to a one-dimensional array of elongated dimples.

times are on the order of a few ten ms and can be approximately doubled with respect to the dark dipole trap by introducing cavity cooling with a weak near-resonant probe. The combination of the red intracavity dipole trap with cavity cooling achieves reliable strong coupling and enabled the first measurement of the normal-mode splitting of a single trapped atom.

In the trap the effective atomic detuning is altered due to the Stark shift. This direct dependence of the effective atomic detuning on the trap depth was deliberately used to measure the anticrossing in the normal-mode spectrum presented in Sec. 8.2 and (94). However, the calibration of the dipole intensity measured in transmission is only possible to within a few percent and in addition to the motion in the standing wave light field, the atomic detuning at the trap center varies with the dipole intensity.

\subsection{The blue trap}

An atom is repelled from blue-detuned light and hence confined to regions of low Stark shift. Hence, the idea of the blue trap is to use far-detuned cavity modes to shape a potential landscape which realizes three-dimensional confinement at a dark trap center (Fig. 3.2 and Fig. 3.3). Such a blue trap has a number of advantages for experiments in cavity QED: (1) Since the trap height does not contribute to the atomic detuning, it can be made large for good confinement. (2) An atom inside the trap is well isolated by the surrounding potential barrier, outside atoms are repelled. (3) The blue trap can 


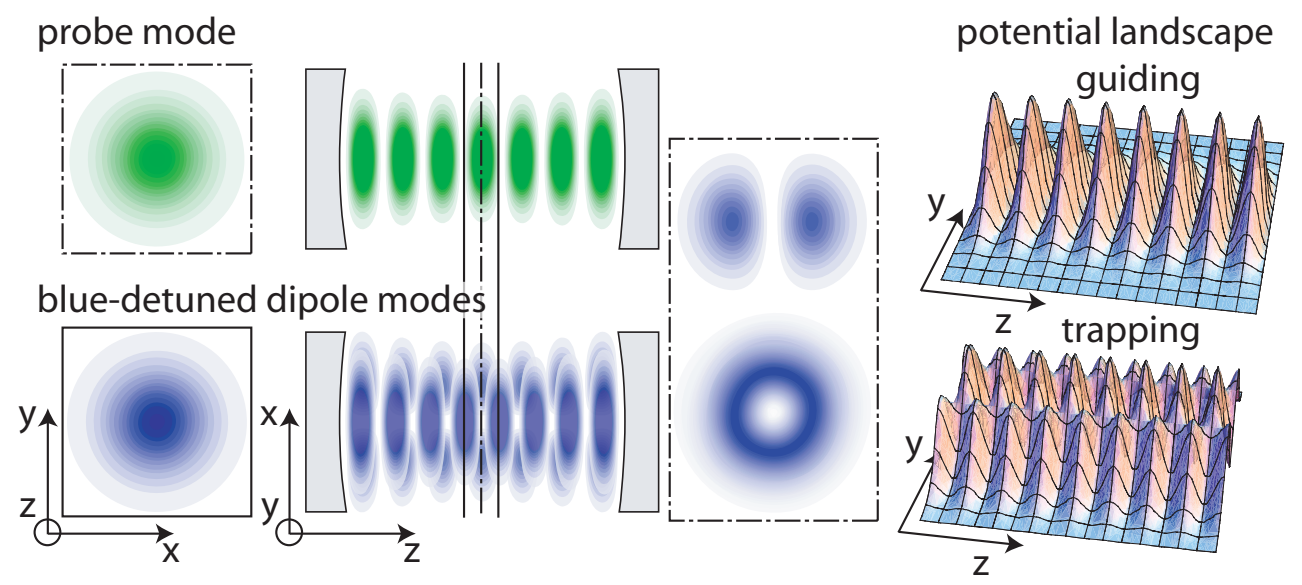

Figure 3.2: Blue intracavity dipole trap. The idea of the blue trap is to use far-detuned cavity modes (blue) to shape a potential landscape which realizes three-dimensional confinement around a dark trap center. The slow atoms are bound to stay in regions of vanishing field intensity. To load an atom, the trap has to be opened. A nodal line guides the atoms to the trap center. Upon detection of an atom strongly coupled to the probe mode (green), the transverse confinement is closed. The energy gain due to guiding and switching is kept small and the atom is stored at a dark trap center largely preserving the free-space properties.

be loaded by creating a dark funnel to guide a slow atom to the trap center. As the atom is repelled from the blue light, the kinetic energy does not increase during the capture process. Moreover, weakly coupled atoms that are not collected by the funnel are rejected. (4) The funnel can be closed upon detection of the strongly coupled atom in the trap center. Because the energy gain due to guiding and switching is kept small, the requirement to cool the atom after the capture process is relaxed. (5) Since during the whole loading sequence the atomic detuning is preserved, parameter regimes of large cavity-enhanced heating $(85 ;$, 86) can be avoided.

The potential landscape of the blue trap consists of mountains insuperable for the slow atoms. The perfectly aligned cavity modes are well suited to generate a onedimensional array of traps. Several higher-order modes of the cavity are combined to create axial and transverse confinement (Fig. 3.2). Persistent axial confinement can be realized analogous to the red case by a TEM 00 mode which is an odd number of FSRs blue-detuned. This field constitutes a shifted array of repellent oblate antinodes, resembling a stack of pancakes. In this case the nodes overlap with the antinodes of the probe field at the cavity center. transverse confinement can be realized by a (combination of) higher-order mode(s) with zero field on the cavity axis, transversely completely surrounded by high intensity regions, e.g. a combination of $\mathrm{TEM}_{10}+\mathrm{TEM}_{01}$ modes forming a doughnut mode. To be aligned with the probe maxima, the modes are an even number of FSRs blue-detuned. The high-finesse cavity guarantees maximum contrast of the interference pattern. The quality of the destructive interference at a node in the standing wave mode is extremely good. A residual imbalance of the counter-propagating waves 
can only be induced by the single sided pumping of the cavity mode and different losses in the mirror coatings. These effects given by the mirror parameters are in the order of a few parts per million. By symmetry, the higher order mode used for the radial confinement has a perfect nodal line in the cavity center. The trap center is therefore accurately dark. Since the kinetic energy of the atoms is small compared to the potential barriers formed by the blue-detuned light fields, atoms are bound to stay in the regions of vanishing blue light intensity. To load an atom, the trap has to be opened. This is achieved by a nodal line of a higher-order mode oriented in the direction of the arriving atoms. Upon detection, only the transverse confinement has to be closed while the axial confinement is preserved. Because the transverse motion of the atom is monitored by the cavity transmission, the energy gain due to switching can be minimized. Since at the trap center the atom is stored at zero intensity, guiding is not accompanied by an increase in kinetic energy and the free-space properties of the atom at the trap center are preserved.

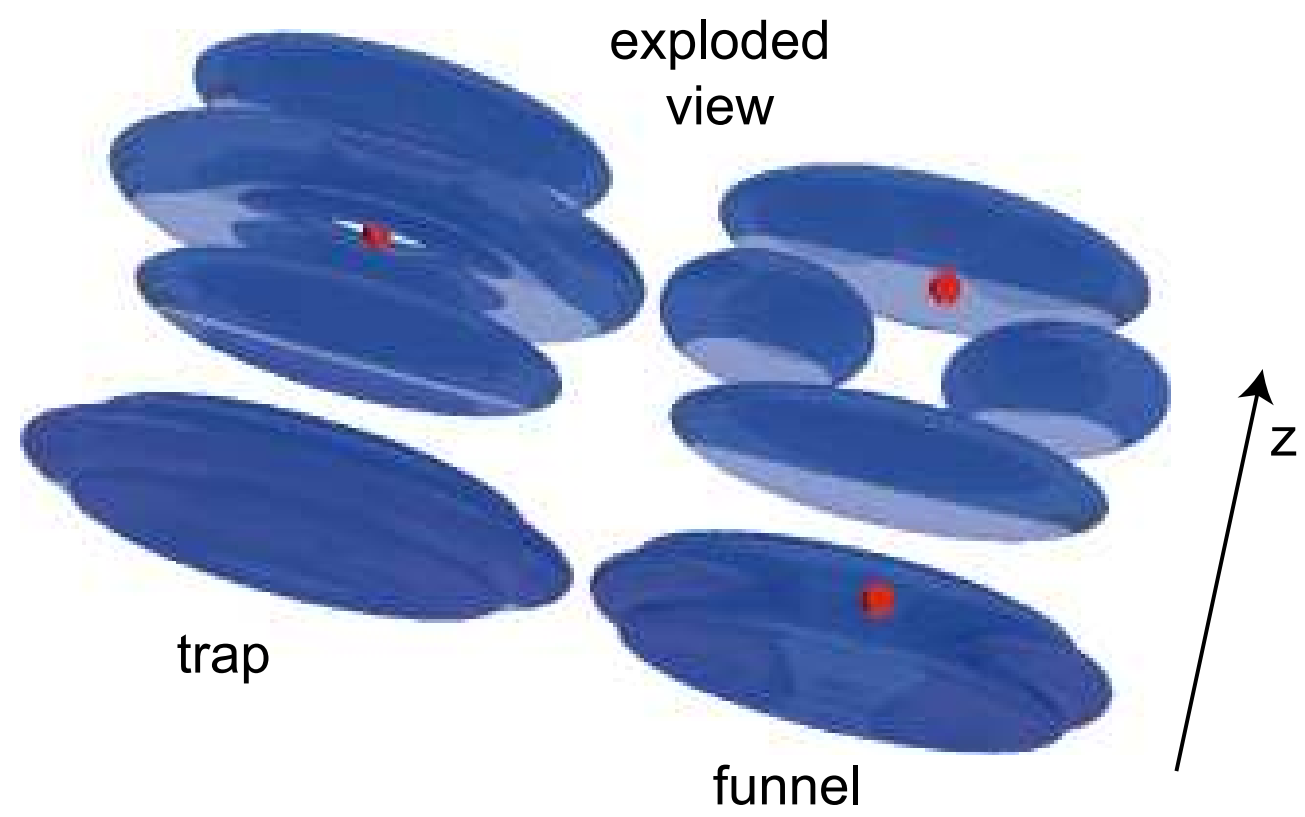

Figure 3.3: The vicinity of the trap center for trapping (left) and guiding (right). Because the trapped atom is completely surrounded by blue-detuned light, an exploded view shows the atom sitting close to the dark center of the doughnut. 



\section{Chapter 4}

\section{Cooling and detection}

All conventional methods of laser cooling rely on repeated cycles of optical pumping and spontaneous emission of a photon. Spontaneous emission provides the dissipative mechanism required to remove entropy, while each emission imprints a recoil in a random direction. Cavity cooling is an alternative method to cool a particle strongly coupled to a high-finesse cavity $(90 ; 01 ;$ 92). Instead of exciting the atom, the photon can be emitted from the cavity. Appropriate parameters for cavity cooling are chosen such that: (1) the emitted photon is blue detuned with respect to the absorbed and (2) the excitation is predominantly exchanged via the cavity contribution to the dressed states.

This chapter introduces two experimentally relevant parameter regimes that allow for axial cavity cooling, but differ with respect to the position dependence of the forces and are referred to as nodal- and antinodal-cooling (Sec. 4.1). The character of the detection also differs between the two regimes (Sec. 4.2). In cooling region I (CRI, Sec. 4.1.1), resonant detection is very robust and provides a high signal to noise ratio. In cooling region II (CRII, Sec. 4.1.2), off-resonant detection allows to discriminate and qualify high couplings. If we consider pumping the system with a laser exciting the atom from the side, the corresponding parameters for the atom and the mode change their roles. Therefore, only cooling region II overlaps with its analog for side pumping and hence, qualifies for the implementation of three-dimensional cavity cooling.

Cavity cooling in the axial direction was first observed in this experiment (88) and is introduced in Sec.4.1. The three-dimensional extension of cavity cooling was realized in a different experiment in the group (97).

\subsection{Cavity cooling}

The velocity-dependent force along the cavity axis to the linear order in velocity can be expressed by the friction coefficient $\beta$. Figure 4.1 shows the friction coefficient $\beta$ as a function of the laser detuning with respect to the bare atom $\Delta_{\mathrm{a}}$ and cavity $\Delta_{\mathrm{c}}$, respectively. A possible Stark shift due to the trap is neglected. In Fig. 4.1 A., the friction coefficient is averaged over the vicinity of the antinode of the probe mode $([-\lambda / 10, \lambda / 10])$, i.e. as appropriate for a well-coupled atom. A scan of the laser corresponds to a diagonal 

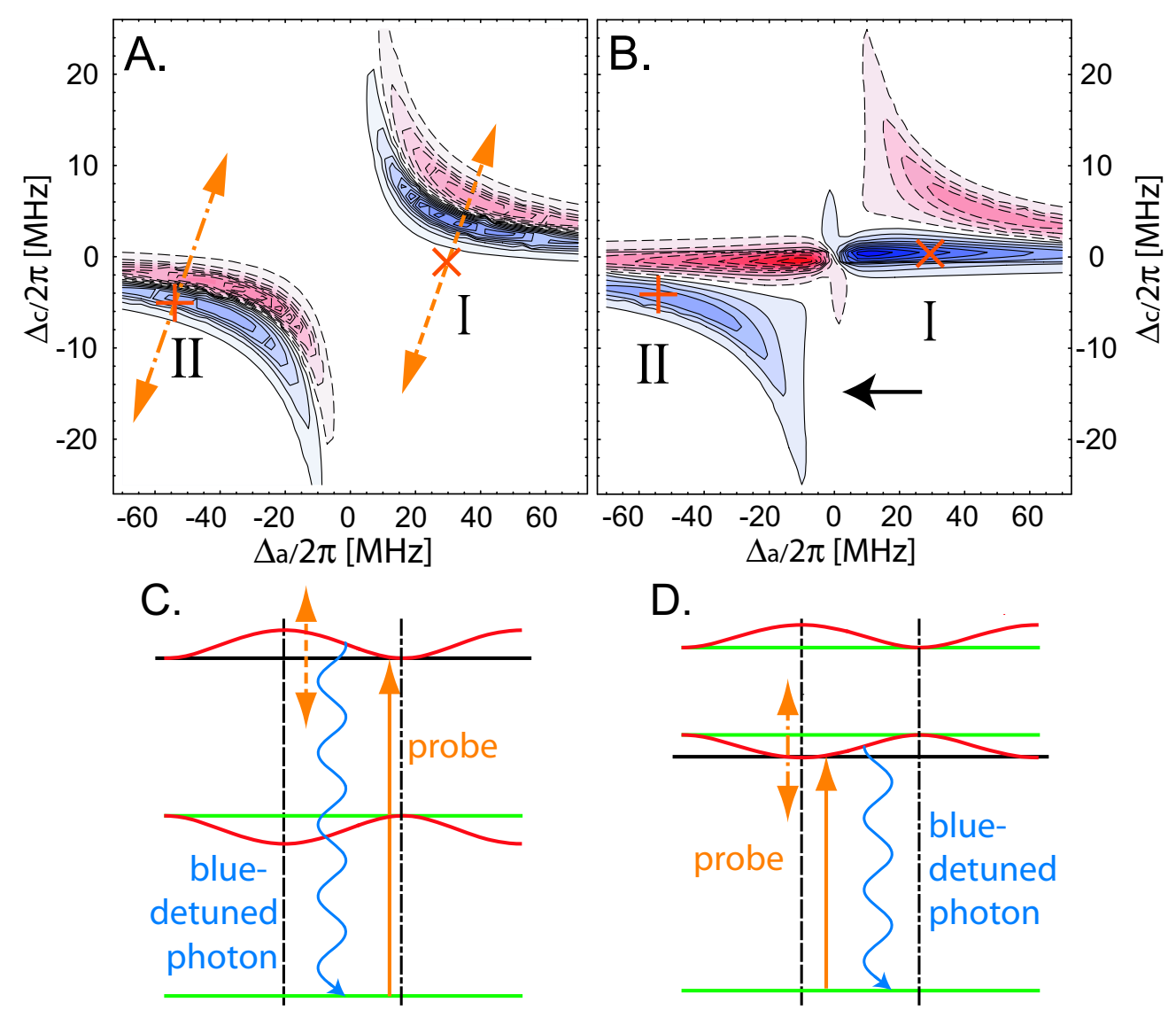

Figure 4.1: Friction coefficient: Nodal cooling (cross) and anti-nodal cooling (plus). A. Friction coefficient averaged over $\pm \lambda / 10$ in the vicinity of an antinode, i.e. trap center. B. Friction coefficient averaged over the a full period along the standing wave direction. C. \& D. Sisyphus cooling cycles in the dressed states along the cavity axis: the system undergoes multiple excitations and emissions. The extra energy of the bluedetuned photons emitted is extracted from the kinetic energy and cools the atomic motion. Heating occurs when one of the modulated dressed states is excited at its maximum. Scanning the probe laser will hence result in strong cooling and heating in the vicinity of the normal modes.

in the two dimensional plot for a given atom-cavity detuning. Starting from negative detunings, such a scan hits two pairs of cooling and heating regions. These cooling (heating) regions, situated on the red(blue)-detuned slope of the normal mode, correspond to the 'Doppler-type' cooling (heating) on the resonances of the coupled system. For the symmetric case, $\Delta_{\mathrm{c}} \equiv \Delta_{\mathrm{a}}$, both normal modes consist of equal contributions of the bare states and are equally well excited. For $\left|\Delta_{\mathrm{c}}\right|<\left|\Delta_{\mathrm{a}}\right|$, the laser is closer to the normal mode that is 'cavity like' and the excitation of the system is stronger when pumping the cavity. Since the excitation is mainly exchanged via the cavity mode, this is where the advantage of cavity cooling is effective, i.e. cooling forces are large compared to free-space at equal excitation of the atom. 
If the spatial interval for averaging is enlarged, the same picture like Fig. 4.1 A. for a slightly less coupled atom is added. Due to the reduced coupling, the normal modes and hence the cooling and heating regions move towards the origin. The outer regions of the lesser coupled atom largely compensate the corresponding inner regions of the stronger coupled. Averaging over the full standing wave only the extreme regions survive, see Fig. 4.1 B., all heating and cooling contributions that lie in between are canceled. The remaining regions can be attributed to nodal cooling (heating): $\left|\Delta_{c}\right|<\kappa, \Delta_{\mathrm{a}}>0$ $\left(\left|\Delta_{\mathrm{c}}\right|<\kappa, \Delta_{\mathrm{a}}<0\right)$ and antinodal cooling (heating): $\Delta_{\mathrm{a}}<\Delta_{\mathrm{c}}<0\left(\Delta_{\mathrm{a}}>\Delta_{\mathrm{c}}>0\right)$.

The trapping potential confines the atom to regions of strong coupling. If the atom moves away from the trap center the coupling is reduced and at the same time the atomic detuning is shifted to lower values when the detuning $\Delta_{\mathrm{a}}$ is taken at the trap center, i.e. including the Stark shift in the red trap. The additional shift during the averaging leads to nodal heating and cooling regions on the $\Delta_{\mathrm{c}}=0$ axis that correspond to the free-space detunings, and antinodal heating and cooling regions that are positioned with respect to the coupled detuning, i.e. the origin.

The preceding analysis thus identifies two cooling regions which are discussed in more detail:

\subsubsection{Cooling region I $\left(\left|\Delta_{\mathbf{c}}\right|<\kappa, \Delta_{\mathbf{a}}>0\right)$}

In cooling region I (CRI, cross in Fig. 4.1) the probe laser is on resonance with the empty cavity, see Fig. 4.1 C.. The system is predominantly excited when the atom is uncoupled at the node. Due to the finite response time given by the decay rates $(\kappa, \gamma)$, the photon is emitted after the system has followed the upper dressed state for some time. Since due to the coupling the dressed states repel each other, the emitted photon is blue detuned. The extra energy is extracted from the kinetic energy of the atom which is cooled. This cooling cycle repeats multiple times in this Sisyphus-type cooling (85). Note that the cooling is most effective for an atom that reaches the node and ceases for a well-coupled atom. Then again the probe excites the bare cavity which leads to very effective cooling at low atomic excitation, i.e. a good ratio of cooling rate to heating by spontaneous emission. Experimentally, a cooling rate at least five times larger than for free-space cooling at equal atomic excitation was observed.

\subsubsection{Cooling region II $\left(\Delta_{\mathrm{a}}<\Delta_{\mathrm{c}}<0\right)$}

In cooling region II (CRII, plus in Fig. 4.1) the probe laser is resonant with the lower dressed state in the coupled case, see Fig. 4.1D.. The cooling can again be understood as multiple Sisyphus type cooling cycles. Here, cooling is effective at the antinode, i.e. for a well-coupled atom. In this parameter regime the probe laser is red detuned with respect to both normal modes. To allow for effective cavity cooling the lower dressed state must be cavity-like. Then, the excitation is mainly transferred via the cavity contribution to the normal mode and the excitation of the atom is kept low.

If we consider pumping the system via the atom with a laser from the side $\left(\eta_{\mathrm{a}} \neq 0\right)$, the roles of atom and cavity are interchanged. Hence, the plot of the friction coefficient 
Fig. 4.1 A. is then given by the mirror image with respect to the diagonal $\left(\Delta_{\mathrm{c}} \equiv \Delta_{\mathrm{a}}\right)$. Therefore, an overlap of the resulting cooling regions is only given for the CRII. Moreover, in CRII the laser is red detuned with respect to both normal modes such that any (offresonant) excitation of the system by energy conservation can only lead to cooling. Hence, this parameter regime allows for cavity cooling in three dimensions (Sec. 7.9).

\subsection{Resonant and off-resonant detection}

One characteristic difference between the two cooling regions is that the probe is resonant with the bare cavity in CRI and resonant with the strongly coupled cavity-like dressed state in CRII. This also results in a distinct difference in the detection of an atom, see Fig. 4.2, When the probe is resonant with the empty cavity as in CRI, the presence of an atom will lead to a decrease in transmission. For any detuning on the order of the maximum coupling $\left|\Delta_{\mathrm{a}}\right| \lesssim g_{0}^{2} / \kappa$ (cf. eqn. (2.16) ), the induced shift of the cavitylike state will be in the order of the linewidth, resulting in a steep drop in transmission. Therefore, the detection of an atom is very robust. Moreover, since the atom switches off the light, the excitation of the coupled system is reduced.

In CRII the probe is resonant with the coupled system (dashed-dotted). The presence of a coupled atom switches the light on and the excitation of the system is increased. This off-resonant detection allows to discriminate between large couplings. The mapping between coupling and transmission can be tailored: A larger atom-cavity detuning reduces the shift induced by the coupling leading to a smoother transition. In CRII the probe detuning with respect to the maximum shift defines which part of the normal mode that is swept with increasing coupling.

\subsection{Cooling regions and Stark shift}

Figure 4.3 shows the dressed-state Sisyphus picture of cavity cooling for the different cooling regions in the dipole traps. For a fixed trap depth two completely analogous dressed state configurations with respect to the cavity axis can be found for the red and blue-detuned traps at appropriately chosen bare detunings. The variation of a dressed state due to changes in the trapping field intensity vanishes close to the freespace detunings. The obvious difference between the red and the blue dipole trap is the position where the free-space detunings are preserved, i.e. the Stark shift is zero. In the red trap this is the case at the node, where the system is uncoupled. The advantage of the blue trap is that the free space detunings are preserved at the antinode which is the target position for a well-coupled system. Note that even though the difference appears small for the CRII, cooling and detection sensitively depends on the detuning at maximum coupling, i.e. on the order of $\kappa$. Experimentally trapping in CRII was not realized in the red-detuned dipole trap, because the Stark shift sweeps the atom detuning across a heating region during the loading. Successful trapping of atoms in the blue-detuned intracavity dipole trap in CRII is presented in Sec. 7.9 . 


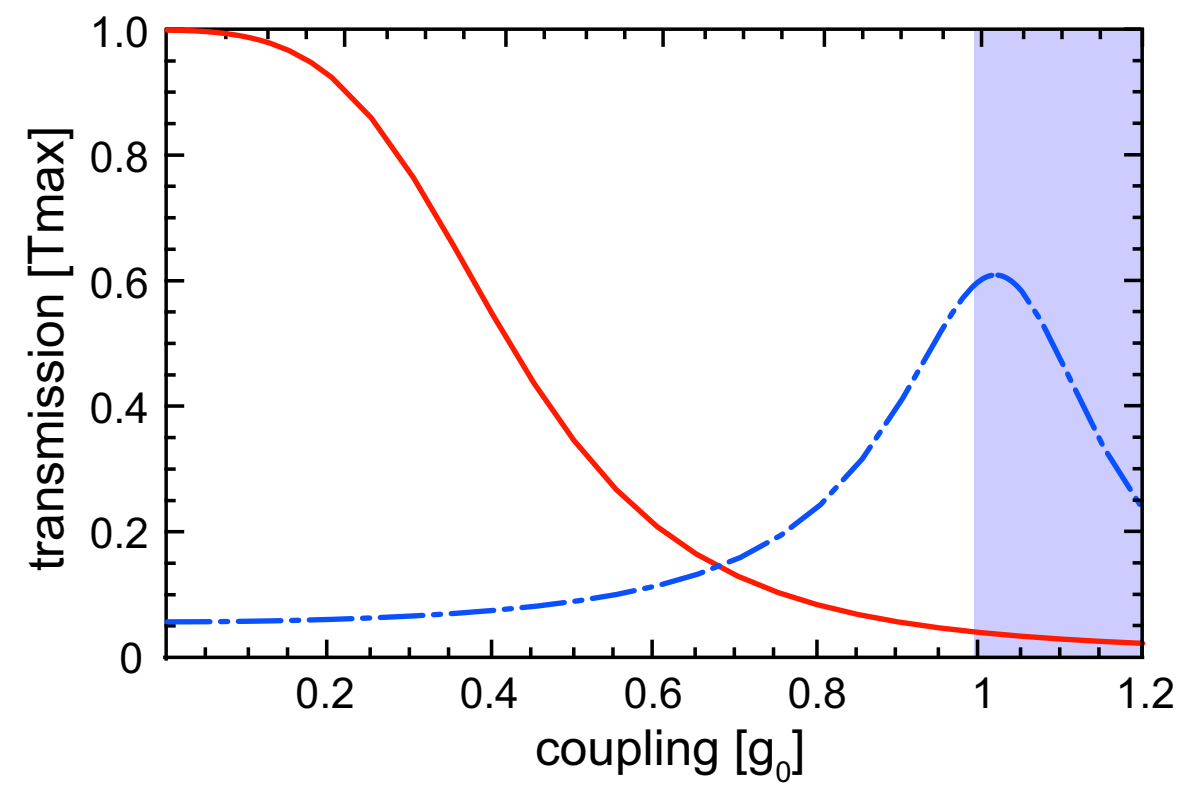

Figure 4.2: Resonant and off-resonant detection. The probe is resonant with the bare cavity (red, solid). The coupled system is tuned out of resonance with the probe such that the excitation and hence the transmission of the cavity drops steeply, when the shift exceeds the linewidth. Resonant detection allows for a high signal to noise and is very robust. In off-resonant detection the coupled system is tuned into resonance with the probe (blue, dash-dotted). The part of the normal mode that is swept with increasing coupling is defined by the probe detuning. Typically, the bare detuning is chosen such that the probe is resonant for maximum coupling. In this case the excitation of the system increases monotonously with increasing coupling. It allows to distinguish between high couplings. 


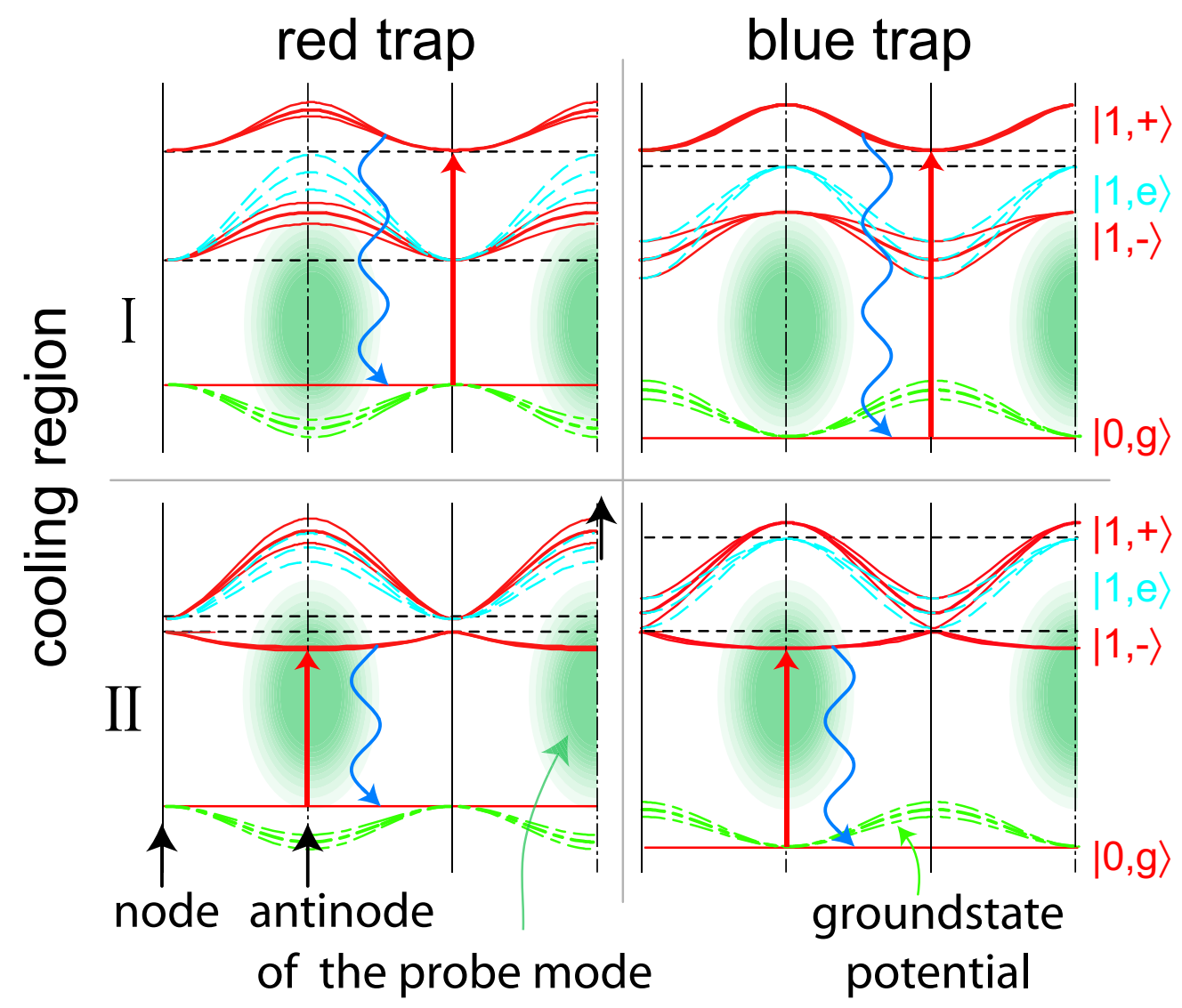

Figure 4.3: Cooling regions in dipole traps: Sisyphus picture of cavity cooling along the cavity axis in the red- and blue-detuned dipole traps for the different cooling regions. The bare states are shown as horizontal lines. The trap is introduced with a corresponding Stark shift: the modulated ground state potential is shown in green (dash-dotted). To simplify the energy balance in the Sisyphus picture, the Stark shift in the atomic transition is entirely attributed to the atomic excited state (blue, dashed). Finally, the coupling is included and the dressed states (red, solid) are obtained. To show the effect of variations in the trap depth two neighboring configurations of different trap depths are shown. Note that the variation vanishes close to the free-space detunings. The obvious advantage of the blue trap is that this position is at the antinode corresponding to a well-coupled system. 


\section{Chapter 5}

\section{Numerical simulation}

Numerical simulations can be considered as the link between experiment and theory. The theory presented in Chap. 2 can be solved analytically for an atom at rest in the limit of low excitation and lowest order in the velocity. Moreover, the light forces and the diffusion are strongly position dependent via the coupling and the Stark shift. Therefore, the spatial distribution of atoms is the result of a complicated motion, which is obtained by numerical simulation. In this chapter the results of the numerical simulations of atoms guided and trapped in blue intracavity light fields are presented and discussed, see also (114). The properties and advantages of the blue intracavity dipole trap identified in (Chap. 3) are confirmed. In particular, the possibility to access the cooling region II (CRII) is supported. The results are used to identify the interesting parameter regimes for the experiments discussed in chapter 7.

The development of numerical simulation for this experiment are reviewed in Sec. 5.1 before introducing the algorithm is introduced in Sec. 5.2. The rest of the chapter will present the results of the numerical trajectory simulations in both the cooling regions I and II (Sec. 5.3.

\subsection{Background}

The progress of the experiment has been accompanied by the development of numerical simulations, which show remarkable quantitative agreement with the experimental data. In the simulations all physical quantities can be calculated, also those which are experimentally not accessible. The ability to perform numerical simulations has been a key to the understanding of the experimental observations and the underlying physics: the different contributions of forces and diffusion to the autocorrelation of the transmitted intensity when atoms are traversing the mode were identified (115; 116). Guiding and trapping atoms in the near-resonant probe field (84) as well as in a far red-detuned mode has been studied. The simulations were extended to account for the presence of a red-detuned intracavity dipole trap. In the 'dark' dipole trap - without probe light storage times are limited by parametric heating due to fluctuations of the dipole field. When the simulated parametric heating is adapted to the losses observed in the dark 
trap, good quantitative agreement is found for the dependence of cavity cooling on the power of the near-resonant probe (88). The simulations show that the onset of axial cooling is reflected in a change in the preferred loss channel. Cavity cooling overcompensates axial parametric heating by the trap light, but the increasing number of recoils from spontaneous emission result in radial losses. The normal-mode spectra of a qualified strongly-coupled atom-cavity system close to the anticrossing in transmission and excess loss rate are reproduced by the simulations, see Sec. 8.2. The simulations allow to verify that the qualification (see Sec. 7.6) completely eliminates the contributions of low coupling $g$.

For the numerical studies of the blue trap, the extra light fields with their spatial dependencies were added to the simulation. The Stark shift of the blue-detuned lasers is calculated taking into account transitions to higher excited states of the atom. In the experiment all dipole lasers are independently locked to the same transfer cavity. Therefore, it is reasonable to assume the parametric heating to be the same as for the red laser. This fixes all additional parameters in the simulations.

\section{$5.2 \quad$ Algorithm}

At low excitation the steady state of the atom-cavity-trap system is solved analytically for an atom fixed in space at a given coupling and developed to first order in velocity (Chap. 2). The analytical expressions allow for efficient, three-dimensional simulation of sample traces based on a trajectory propagation. Further details on the numerical simulations can be found in (87).

The atom is well described by a point-like particle, because at the temperatures considered its de Broglie wavelength is much smaller than the optical wavelength. A moving atom is subject to conservative dipole forces of the light fields, velocity-dependent forces due to the delayed adjustment of the system to changes of position dependent parameters and diffusion caused by random momentum kicks. Given the steady-state expectation values, the Newtonian equations of motion for the position $\mathbf{r}(t)$, and momentum $\mathbf{p}(t)$ can be written as

$$
\begin{aligned}
\dot{\mathbf{r}}(t) & =\frac{\mathbf{p}(t)}{m_{\mathrm{Rb}}} \\
\dot{\mathbf{p}}(t) & =\left\langle\mathbf{F}_{d p}(\mathbf{r}(t))\right\rangle+\left\langle\mathbf{F}_{v}(\mathbf{r}(t), \mathbf{p}(t))\right\rangle+\dot{\mathbf{p}}_{d p}(\mathbf{r}(t))+\dot{\mathbf{p}}_{s e}(\mathbf{r}(t)) .
\end{aligned}
$$

Here, $\left\langle\mathbf{F}_{d p}(\mathbf{r}(t))\right\rangle$ and $\left\langle\mathbf{F}_{v}(\mathbf{r}(t), \mathbf{p}(t))\right\rangle$ are the expectation values for the dipole force and the velocity-dependent force, respectively. They are completely defined by the current position $\mathbf{r}(t)$ and momentum $\mathbf{p}(t)$ of the atom.

The diffusive heating mechanisms are represented by stochastic processes. The effect of spontaneous emission and fluctuations of the dipole force 2.36) are modeled by the random momentum kicks $\dot{\mathbf{p}}_{s e / d p}(\mathbf{r}(t))$. The dipole force as well as the momentum diffusion $D_{d p}(2.60)$ preferentially act in the axial direction, because they are proportional to the gradient of the coupling. The gradient is proportional to the typical inverse 


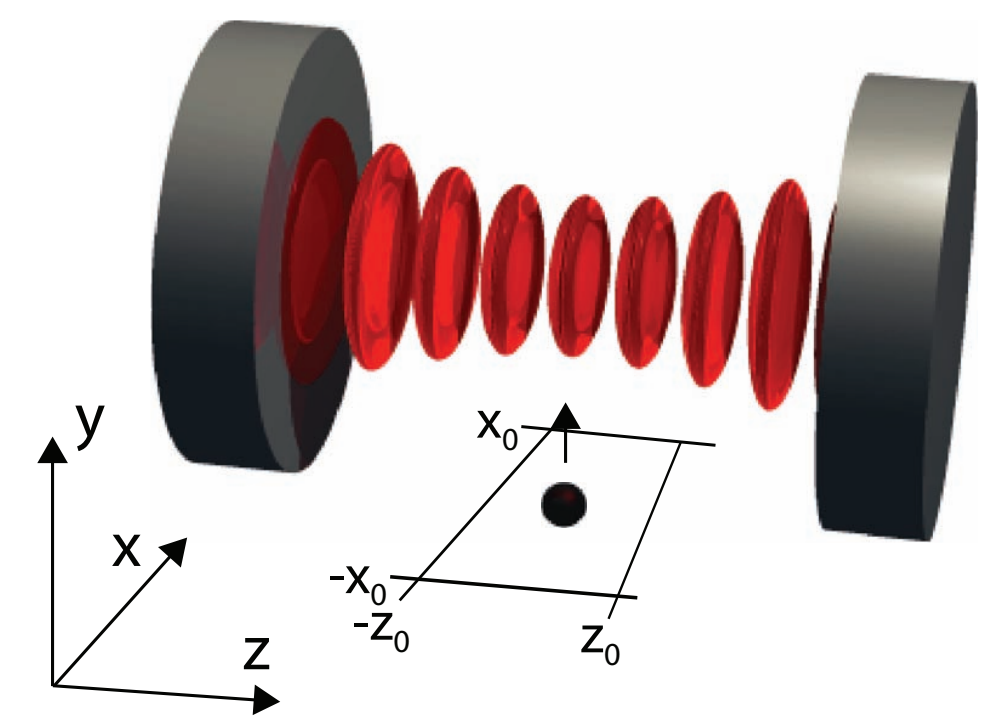

Figure 5.1: Initial conditions in simulations. The atoms are started at $y_{0}=-2 w_{0}$ below the cavity center with a randomly chosen position in the $x-z$ plane in symmetric intervals of $x \in\left[-x_{0}, x_{0}\right] \times w_{0}$ and $z \in\left[-z_{0}, z_{0}\right] \times \lambda$.

length scale, which is $\frac{w_{0}}{\lambda / 2} \approx 60$ times larger in the axial direction. The implementation of the diffusion due to spontaneous emission $D_{s p}(2.59)$ takes the dipole emission pattern into account by reproducing the probability distribution of the recoils. The ${ }^{85} \mathrm{Rb}$ atom is optically pumped to the $5 S_{1 / 2}, F=3, m_{F}=3$ state and excited on the $5 S_{1 / 2}, F=3, m_{F}=3 \rightarrow 5 P_{3 / 2}, F=4, m_{F}=4$ transition with circularly polarized light. The diffusion by spontaneous emission is therefore $\frac{2}{5}$ in the direction of the quantization axis and $\frac{3}{10}$ in both orthogonal radial directions. The parametric heating with contributions from all dipole lasers is included by a stochastic rescaling of the Stark shift and the expectation value of the dipole force of the trap fields $\left\langle\mathbf{F}_{\mathrm{dp}, \operatorname{trap}}(\mathbf{r}(t))\right\rangle$, see Sec. 2.7. The rescaling leads to a Gaussian distribution around the expectation value with a width chosen to reproduce the first moment, i.e. the diffusion constant. The experimental origins are both direct intensity variations of the trap beams as well as frequency variations of the lasers, which are converted into intensity variations by the transfer-function of the cavity. The parametric heating is proportional to the noise density at twice the trap frequency and the square of the trap frequency (117). Because of the different scaling $\left(\lambda / 2 \ll w_{0}\right)$, parametric heating in axial direction is two orders of magnitude larger than in radial direction.

The initial conditions in the simulations (Fig. 5.1) are chosen to closely mimic the experiment (Chap. 6). Atoms are started at a random position in the $(x-z)$ plane, in symmetric intervals of $x \in[-0.4,0.4] \times w_{0}$ and $\left.z \in[-20,20] \times{ }^{(\text {(i.) }}\right)$ at $y_{0}=2 w_{0}$ beneath the cavity. The vertical velocity is fixed at $v_{y} \approx 0.1 \frac{\mathrm{m}}{\mathrm{s}}$. The horizontal velocity along the cavity axis $z$ is restricted to about one recoil $\left(v_{z}=6 \frac{\mathrm{mm}}{\mathrm{s}}\right)$ by geometrical selection. The

\footnotetext{
(i.) In reality, the length of the cavity corresponds to $156 \times \lambda$.
} 
parameters used in the simulations and their meaning are compiled in table B.1 in the appendix.

The atom is propagated by integrating the equations of motion (5.1, 5.2) using a Runge-Kutta algorithm. To trap the atom, the conservative dipole trap has to be switched upon the detection of a strongly coupled atom. For most measurements the probe is on resonance with the empty cavity such that the atom causes a dip in the transmitted intensity. The transmission is sampled with a low-pass filter with a bandwidth of $100 \mathrm{kHz}$ and the trap is switched when the transmission drops below a preselected threshold. For off-resonant detection the transmission increases with the coupling and correspondingly has to be larger than the trigger level. Finally, the propagation of the trajectory is stopped when the atom hits one of the mirrors or the radial distance exceeds $2 w_{0}$. These two loss directions are represented by the 'exitway'. For axial and radial loss the exitway is defined as exitway $=1$ and exitway $=1$, respectively. After implementing the additional blue-detuned laser fields the numerical simulations are checked to reproduce the results of the simulations for the red trap (88).

In the simulations presented in this chapter, the blue $\mathrm{TEM}_{00}$ mode is only one freespectral range (FSR) detuned at $777 \mathrm{~nm}$. In the experiment a three FSR detuned mode was implemented to facilitate the separation of the light beams in the detection.

\subsection{Results}

This section discusses the numerical simulations of the blue trap. The properties and advantages found in the analytical studies of Chap. 3 are confirmed by the numerical results. As discussed in Sec. 4.2 there is a correspondence between the two parameter regimes for cooling and the preferred way of detection. The discussion starts with the parameters of cooling region I (CRI), also used in the red intracavity dipole trap. Then the possibility to access the second cooling region II (CRII) is addressed.

\subsubsection{Cooling region I $\left(\left|\Delta_{\mathbf{c}}\right|<\kappa, \Delta_{\mathbf{a}}>0\right)$}

The red trap was realized in cooling region I (CRI) $\left(\Delta_{\mathrm{a}}>0, \Delta_{\mathrm{c}}=0\right)$. An increase of the storage time by a factor of two with respect to the dark trap was achieved by applying near-resonant probe light (Fig. 5.2). Since cavity cooling overcompensates axial parametric heating due to intensity variations of the dipole field (117), the exit way changes from axial to radial. In red-detuned laser fields atoms are attracted to the intensity maxima. The guiding field is therefore given by a conservative potential well. 'Rolling' into this well, the atom gains enough kinetic energy to reach any location in the cavity mode. In contrast to the radial motion, the axial motion is much faster than the detection bandwidth and can hence not be resolved. Particularly, when the trap is switched, the atom has an arbitrary position in the standing wave direction. On average the atom gains half the potential depth in axial energy by switching. This energy gain can then be counteracted by axial cavity cooling.

In this cooling region, the probe is on resonance with the empty cavity to excite the 


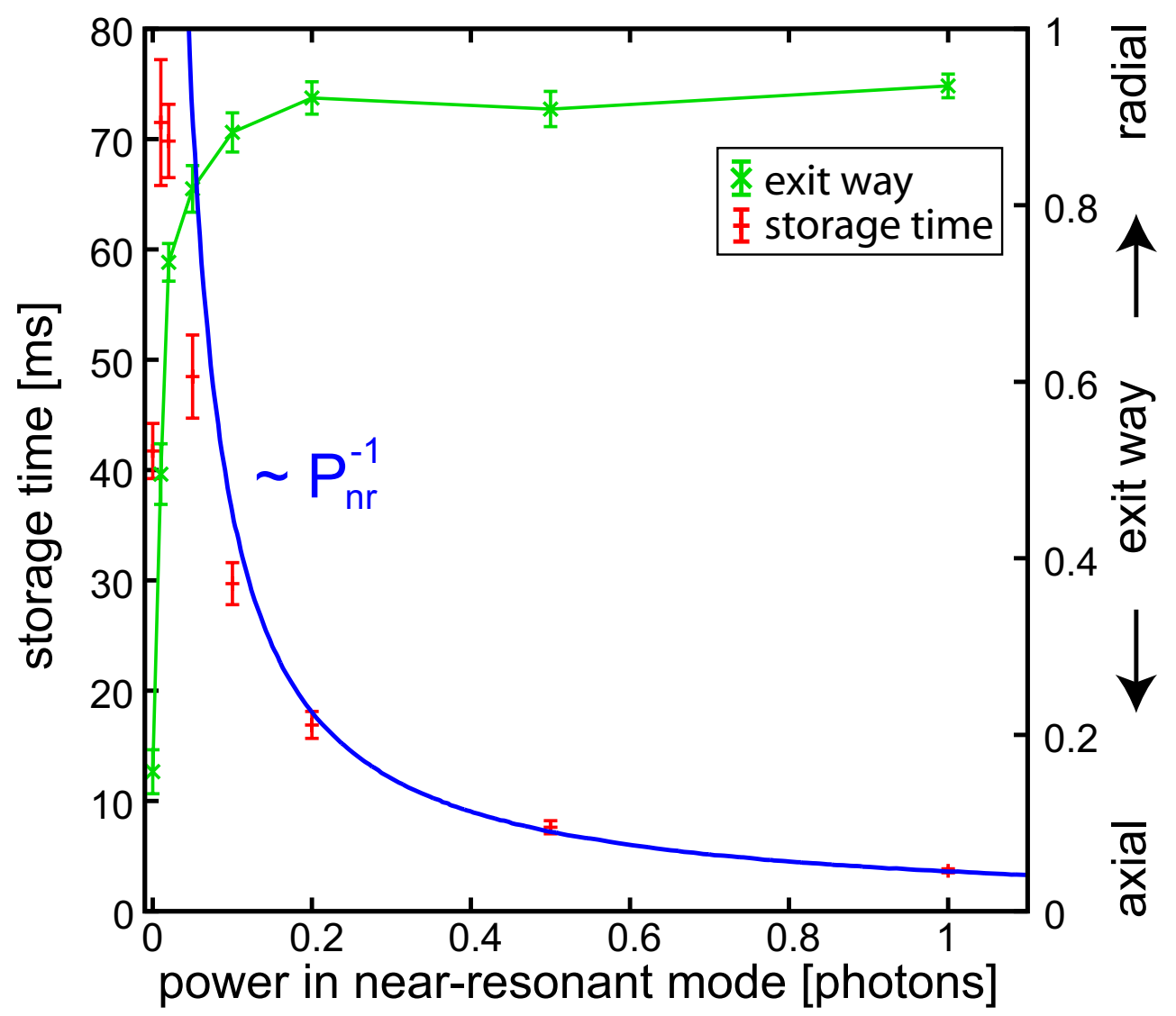

Figure 5.2: Cavity cooling in the red trap. Storage time and exit way in the red intracavity dipole trap versus the power in the near-resonant probe mode. For high probe powers the storage time is inversely proportional to the power $P_{\mathrm{nr}}^{-1}$ (curve). With the axial cavity cooling compensating for the parametric heating due to fluctuations of the trap, the preferred exit way changes from axial (0) to radial (1). $\left\{T_{\text {trig }}=0.1\right.$ (ii.) $P_{\mathrm{nr}}=1 ; x_{0}=[-0.4,0.4] ; y_{0}=-2 ; z_{0}=[-20,20] ;\left(\Delta_{\mathrm{a}}, \Delta_{\mathrm{c}}\right)=(35,0) ;$

$\left.\left(P_{\mathrm{r}}, P_{\mathrm{rs}}\right)=(80,160) \cdot 10^{3}\right\}$. The parameters and their meaning are listed in Appendix $\mathrm{B}$.

upper dressed state in the minimum, i.e. nodal cooling. It is therefore blue detuned with respect to the atom. To compensate for the repellent dipole force a far-detuned red dipole field is used.

Blue-detuned light fields correspond to potential hills. The slow atoms do not have the energy to climb the potential hills and are bound to stay close to zero intensity. A blue $\mathrm{TEM}_{00}$ mode and a $\mathrm{TEM}_{10}$ with the nodal line oriented in the vertical direction can be combined to form 'funnels'. These funnels allow for $2 \mathrm{D}$ guiding of the atoms to regions of strong coupling. Figure 5.3 shows the effect of the guiding fields on the average trigger position of the atom. The guiding field power is given for the 1FSR blue detuned $\mathrm{TEM}_{00}$ mode at $777 \mathrm{~nm}$, the $2 \mathrm{FSR}$ detuned $\mathrm{TEM}_{01}$ guiding mode at $775 \mathrm{~nm}$ is excited 


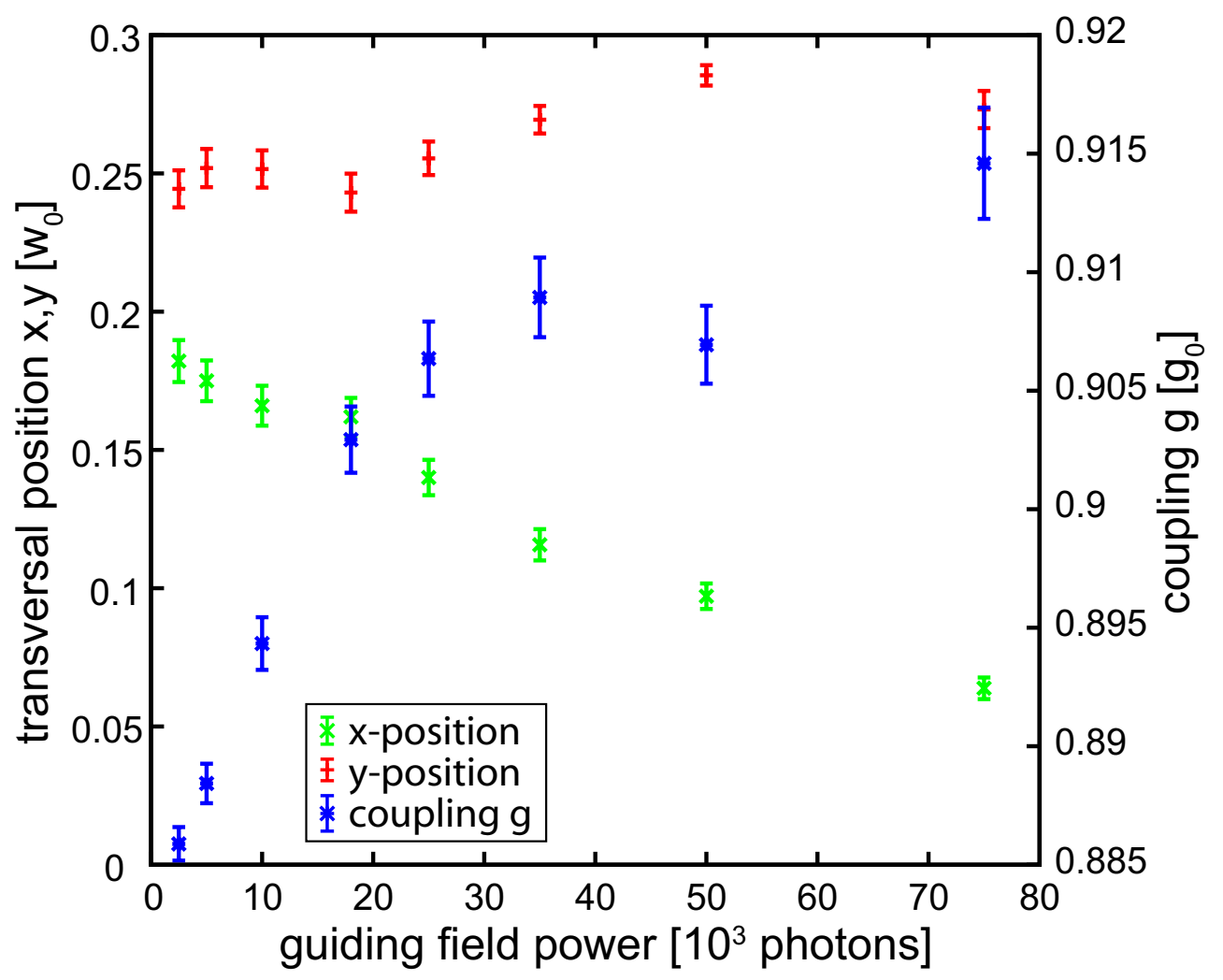

Figure 5.3: Localization with increasing guiding field power: The graph shows the average axial and radial trigger position and corresponding coupling $g$ depending on the guiding field power. With increasing guiding field power, the localization in $x$ direction improves while the $y$ position is given by the trigger value. The guiding field power is given for the $\mathrm{TEM}_{00}$ mode, the power in the $\mathrm{TEM}_{01}$ mode is twice as high. Here, the relevant parameters are $\left\{T_{\text {trig }}=0.04 ; P_{\mathrm{nr}}=1 ; x_{0}=[-0.4,0.4] ; y_{0}=-0.5 ; z_{0}=[-20,20] ; P_{\mathrm{r}}=36 \cdot 10^{3} ;\right.$ $\left.\left(\Delta_{\mathrm{a}}, \Delta_{\mathrm{c}}\right)=(35,0)\right\}$.

at twice the power, $\left(P_{\mathrm{b} 1}=2 P_{\mathrm{b} 0}\right)$. The trigger threshold is set to $4 \%$ of the empty cavity transmission. With increasing field strength in the guiding fields the atoms are more and more restricted to the nodal line. The average position in the $x$-direction is reduced by more than a factor of 2 . With better localization in $x$ the coupling necessary to trigger is reached earlier in the $y$ direction. This explains the small increase in the vertical $y$ direction. The trigger threshold defines the high offset value of the coupling $g \approx 0.9 g_{0}$. Still an increase in the average coupling is observed due to the better localization in the $z$ direction. While the atom enters radially, the funnel centers the atom to the nodal line. It is expected that at some guiding field strength the funnel will be so tight that atoms are reflected. This can be analyzed on the relative trigger probability $P_{\text {trig. }}$. For guiding fields $P_{\mathrm{b} 0} \leq 5 \times 10^{4}$ photons $P_{\text {trig }}>94 \%$, i.e. most of the atoms reach a coupling sufficient to reduce the transmission to the threshold value. For a guiding field of $P_{\mathrm{b} 0}=7.5 \times 10^{4}$ photons the relative trigger probability is reduced to $P_{\text {trig }} \approx 80 \%$. According to Fig. 5.3 


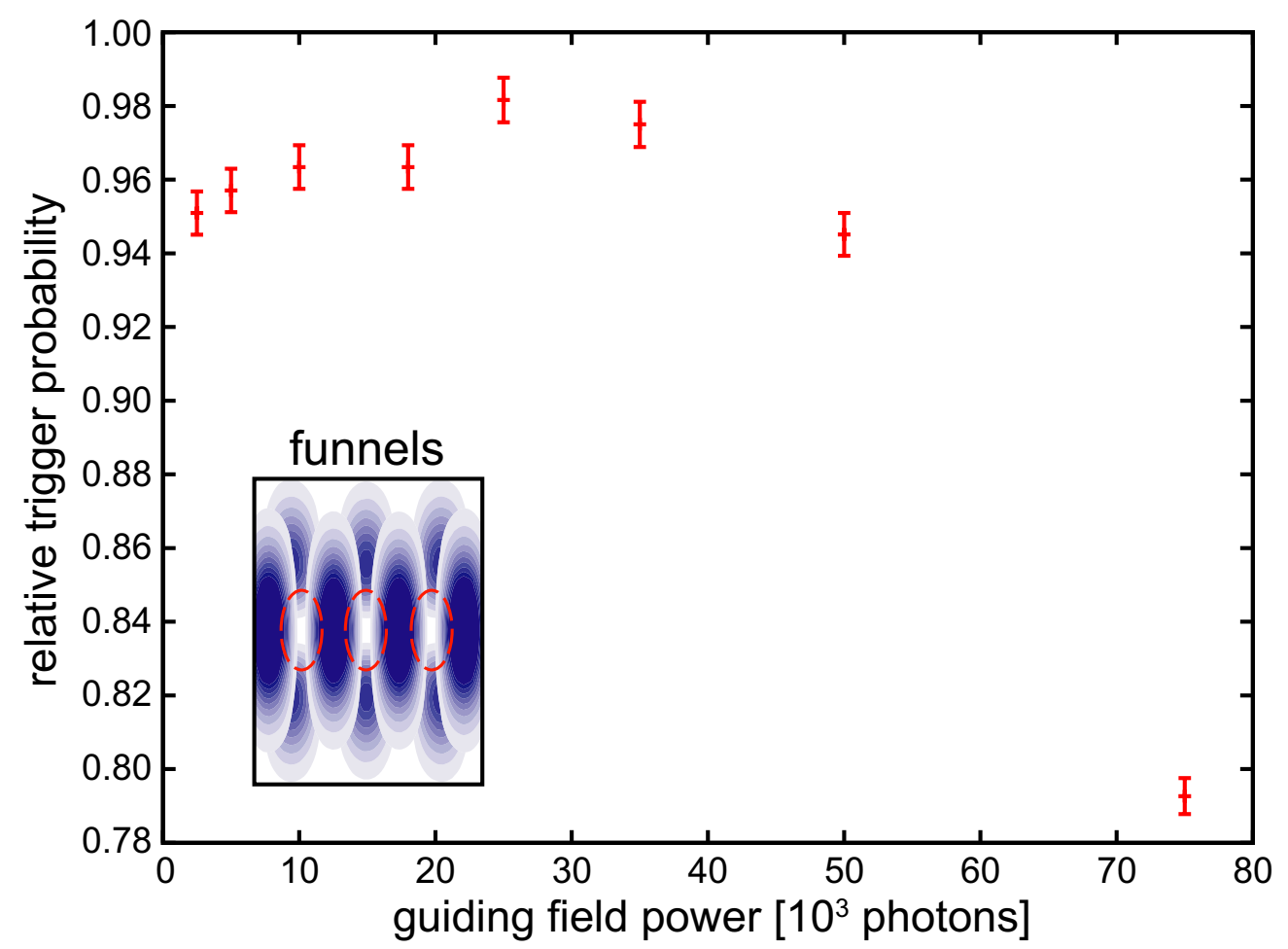

Figure 5.4: Relative trigger probability with increasing guiding field. At very high guiding field intensities the funnels lace up and the atom does not reach the required coupling to trigger before it is reflected. However, the reflection of the atom only happens after significant guiding is achieved, as Fig. 5.3 shows. Parameters are the same as in Fig. 5.3

guiding by the blue-detuned light fields is effective before a significant fraction of atoms is repelled (see Fig. 5.3).

Once the atom is detected, it is trapped by switching the blue-detuned light fields from $\left(P_{\mathrm{b} 0}, P_{\mathrm{b} 1}\right)=(50,100) \times 10^{3}$ to $\left(P_{\mathrm{b} 0 \mathrm{~s}}, P_{\mathrm{b} 1 \mathrm{~s}}\right)=(100,300) \times 10^{3}$ photons and adding the $\mathrm{TEM}_{01}$ mode. The power in the TEM $\mathrm{TE}_{01}$ after switching is the same as for the TEM $\mathrm{TH}_{10}$ mode to form the doughnut. Switching the guiding modes is in principle not necessary, because with the $\mathrm{TEM}_{01}$ mode full 3D confinement is realized. However, a lower guiding intensity avoids the problem of the reduced relative trigger probability and the effect of the switching on the energy of the atom in the vertical direction can be studied. Figure 5.5 shows the signature of cavity cooling in the blue trap. The storage times show an increase by a factor of about three at $P_{\mathrm{nr}} \approx 0.15$ photons. At this intensity the axial, dominantly parametric heating is compensated by cavity cooling. For even higher probe powers storage times decrease as $P_{\mathrm{nr}}{ }^{-1}$, because of radial heating due to spontaneous emission. Compared to the case of the red dipole trap (Fig. 5.2), there are additional points below the maximum storage time for low probe intensity. The change in exit way is not as pronounced in the blue trap, because once the atom is able to escape the trap it will 'roll down' the outer slopes. This will be discussed in more detail on a 


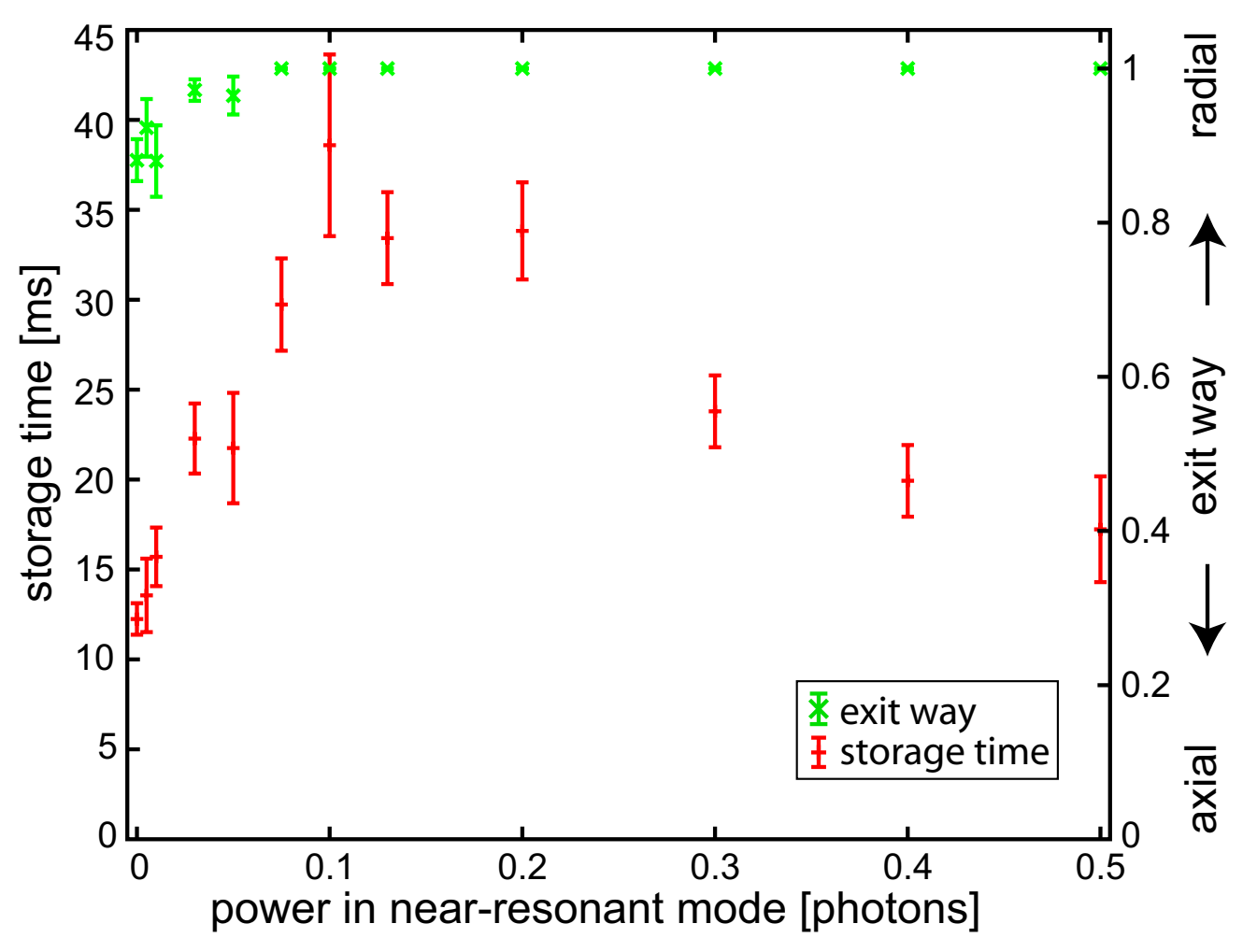

Figure 5.5: Cavity cooling in the blue trap in cooling region I. $\left(\Delta_{\mathrm{a}}>0, \Delta_{\mathrm{c}}=\right.$ 0 ). An increase in storage time as a signature of cavity cooling is observed for low powers of near-resonant probe light. With the onset of cavity cooling overcompensating axial parametric heating due to the dipole trap, the exit way changes to completely radial. $\left\{T_{\text {trig }}=0.04 ; P_{\mathrm{nr}}=1 ; x_{0}=[-0.4,0.4] ; y_{0}=-0.5 ; z_{0}=[-20,20] ; P_{\mathrm{r}}=36 \cdot 10^{3} ;\right.$ $\left.\left(P_{\mathrm{b} 0}, P_{\mathrm{b} 0 \mathrm{~s}}\right)=(50,100) \cdot 10^{3} ;\left(P_{\mathrm{b} 1}, P_{\mathrm{b} 1 \mathrm{~s}}\right)=(150,300) \cdot 10^{3} ;\left(\Delta_{\mathrm{a}}, \Delta_{\mathrm{c}}\right)=(35,0)\right\}$.

sample trace in subsection 5.3 .3 .

\subsubsection{Cooling region II $\left(\Delta_{\mathrm{a}}<\Delta_{\mathrm{c}}<0\right)$}

In cooling region II $\left(\Delta_{\mathrm{a}} \ll \Delta_{\mathrm{c}}<0\right)$ the probe light is guaranteed to be red detuned with respect to both dressed states. By energy conservation this setting can only lead to a velocity-dependent force that is cooling. This cooling region coincides with the corresponding cooling region $\left(\Delta_{\mathrm{c}}<0, \Delta_{\mathrm{a}}<0\right)$ for an excitation of the atom from the side (Sec. 2.7). Exciting the atom from the side leads to cavity cooling in the radial direction. Strong cooling forces are induced by counter-propagating transverse beams with intensity (or polarization) variation on the scale of the wavelength $\lambda$. Guiding and trapping in this cooling region could not be achieved with the red-detuned dipole trap, because due to the Stark shift of the trap the system will generally pass a region of cavity heating in the trapping process.

The cooling region $\left(\Delta_{\mathrm{c}}<0, \Delta_{\mathrm{a}}<0\right)$ is given by the excitation of the lower dressed 


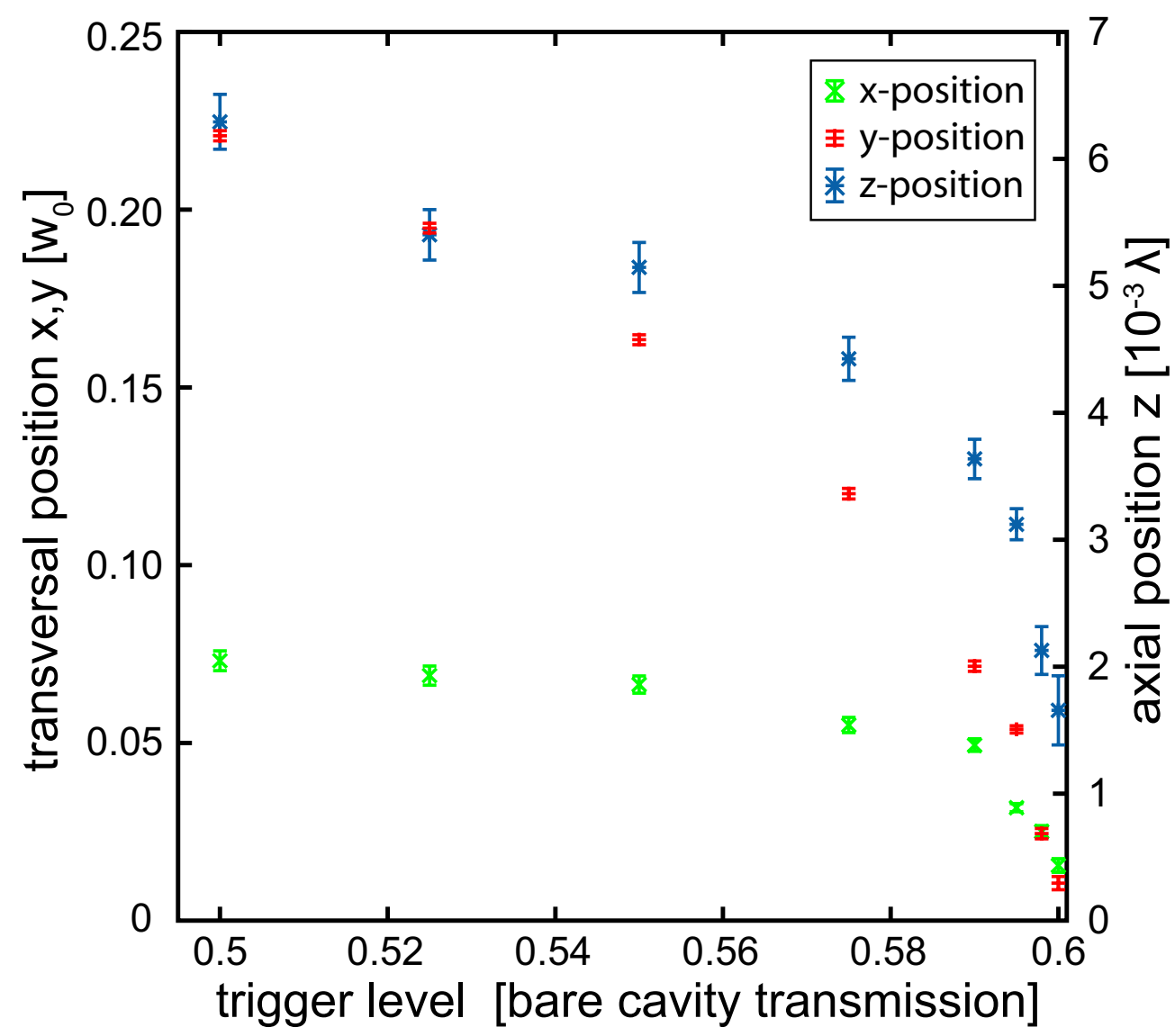

Figure 5.6: Localization depending on the trigger level: With increasing trigger level the atoms are better localized at the cavity center at the trigger time. $\left\{P_{\mathrm{nr}}=1 ; x_{0}=[-0.4,0.4] ; y_{0}=-0.5 ; z_{0}=[-20,20] ; P_{\mathrm{r}}=0 ; P_{\mathrm{b} 0}=10 \cdot 10^{3}\right.$; $\left.P_{\mathrm{b} 1}=20 \cdot 10^{3} ;\left(\Delta_{\mathrm{a}}, \Delta_{\mathrm{c}}\right)=(-45,-6)\right\}$.

state at minimum energy, corresponding to the maximum coupling and hence, 'antinodal' cooling (Sec. 4.1.2). Since the lower bare state is repelled to lower energies, the probe beam has to be red detuned with respect to this bare state. Because cavity cooling relies on the idea of predominantly exciting the cavity part of the system, the lower dressed state must be more cavity-like $\left(\Delta_{\mathrm{a}} \ll \Delta_{\mathrm{c}}<0\right)$. Hence, the lower bare state is the cavity and the probe is red detuned to be resonant with the coupled state.

When the off-resonant cooling laser is at the same time used to detect the atom, the presence of the atom increases the cavity transmission. As discussed, this allows to discriminate high couplings (Fig. 4.2). Figure 5.6 shows the average trigger position depending on the trigger level $T_{\text {trig }}$ in units of the bare cavity transmission. The maximum value of 0.6 corresponds to the transmission on the normal-mode peak. An improved localization is achieved in all directions. Note that, given in natural units, the localization in $z$ is already very good for all trigger values, i.e. the repellant blue axial mode confines 
the atom to the nodal planes. With increasing value of $T_{\text {trig }}$ first $\left(T_{\text {trig }} \lesssim 0.58\right)$ the $y$ position is selected. This corresponds to a later trigger time, waiting for the atom to reach the center of the mode. For $T_{\text {trig }}$ approaching the optimum value there is a strong selection to couplings arbitrarily close to the maximum coupling $g_{0}$ (Fig. 5.7). However, this is accompanied by a strong reduction in the relative trigger probability. A good compromise of a coupling of $0.98 g_{0}$ can be achieved while the trigger probability is close to $P_{\text {trig }} \approx 90 \%$. In the experiment the technical noise on the detection signal will lead to a reduced discrimination capability. Moreover, the delay in the switching of the trap will effectively shift the optimum detection level to lower values.

To study the effects of cavity cooling in the axial direction, again the storage time with respect to the near-resonant probe light is considered (Fig. 5.8). To exclude effects of radial heating, spontaneous emission was switched off in the simulation. Also the diffusion terms due to the dipole-force fluctuations of the far detuned light fields are not included. These scale inversely with the detuning and should therefore be negligible for large detunings. As noted above, the scaling of the parametric heating by fluctuations of the dipole field intensities has been transferred from the well established value in the

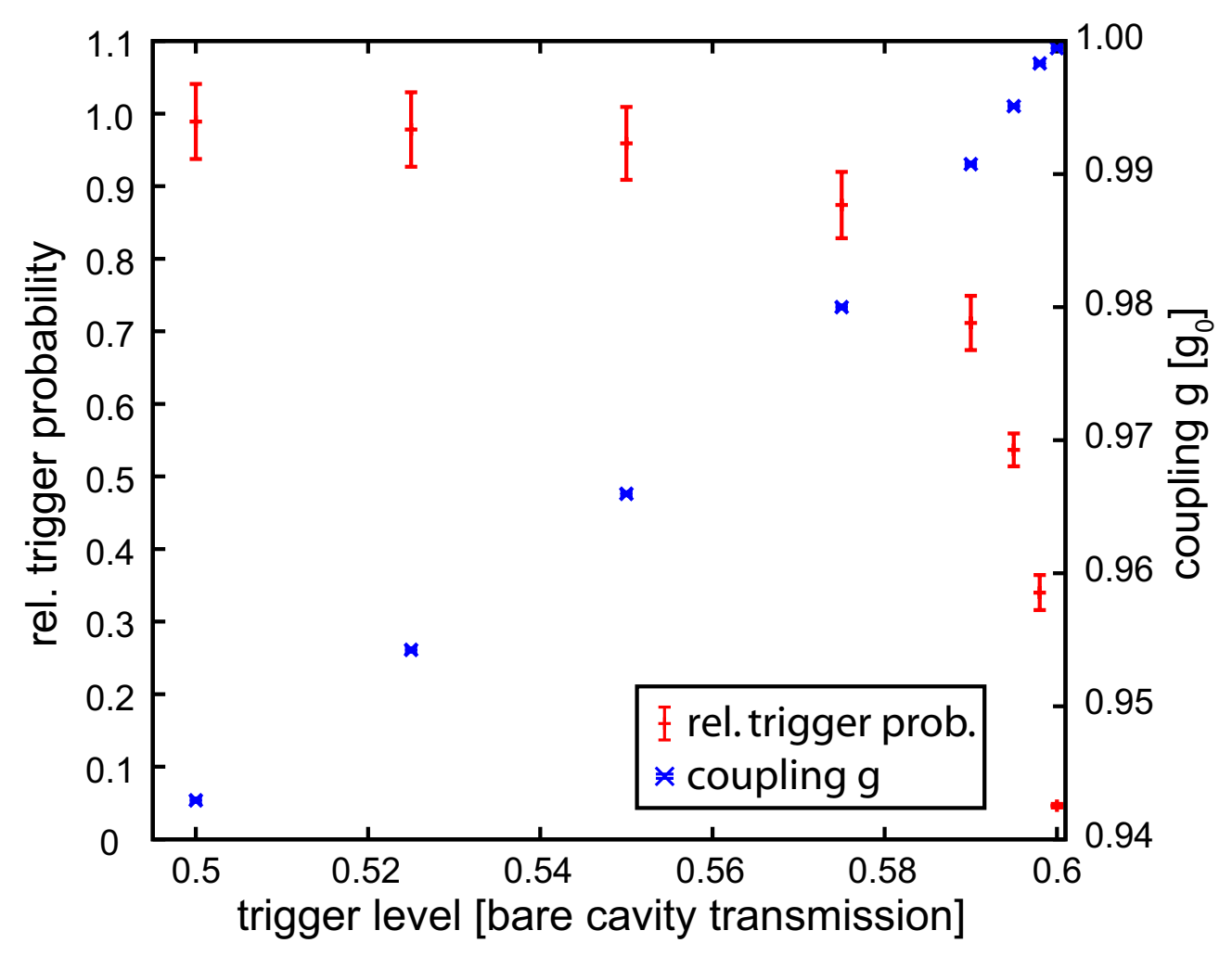

Figure 5.7: Relative trigger probability and coupling as functions of the trigger level. When triggering on increasing transmission, high couplings can be discriminated. For high trigger levels $\left(T_{\text {trig }}>0.58\right)$ atoms with very high couplings are selected and at the same time the relative trigger probability decreases. Parameters are the same as in Fig. 5.6 . 
red trap. However, the fluctuations of this field where chosen to correctly reproduce the axial heating. Therefore, it is not a priori clear that contributions to radial losses are equally well simulated.

An increase in the storage times is observed for very low probe powers. For low probe powers the losses are driven by the parametric heating due to the trap fields and the additional dipole diffusion due to the near-resonant probe. At a probe intensity of $P_{\mathrm{nr}}=1 \times 10^{-4}$ these effects are overcompensated by cavity cooling. This is confirmed by the change in the preferred exit way. With spontaneous emission included, (magenta point in Fig. 5.8), the storage time is reduced, but the enhancement in storage time remains visible. Adding the diffusion due to the dipole force fluctuation of the fardetuned trap light, the storage time in the dark trap is reduced. No enhancement is obtained; hence, the effect of cavity cooling is masked. The diffusion due to the dipole trap can be reduced by a larger detuning of the trap lasers, as is the case in the experimental realization (see section 7 ).

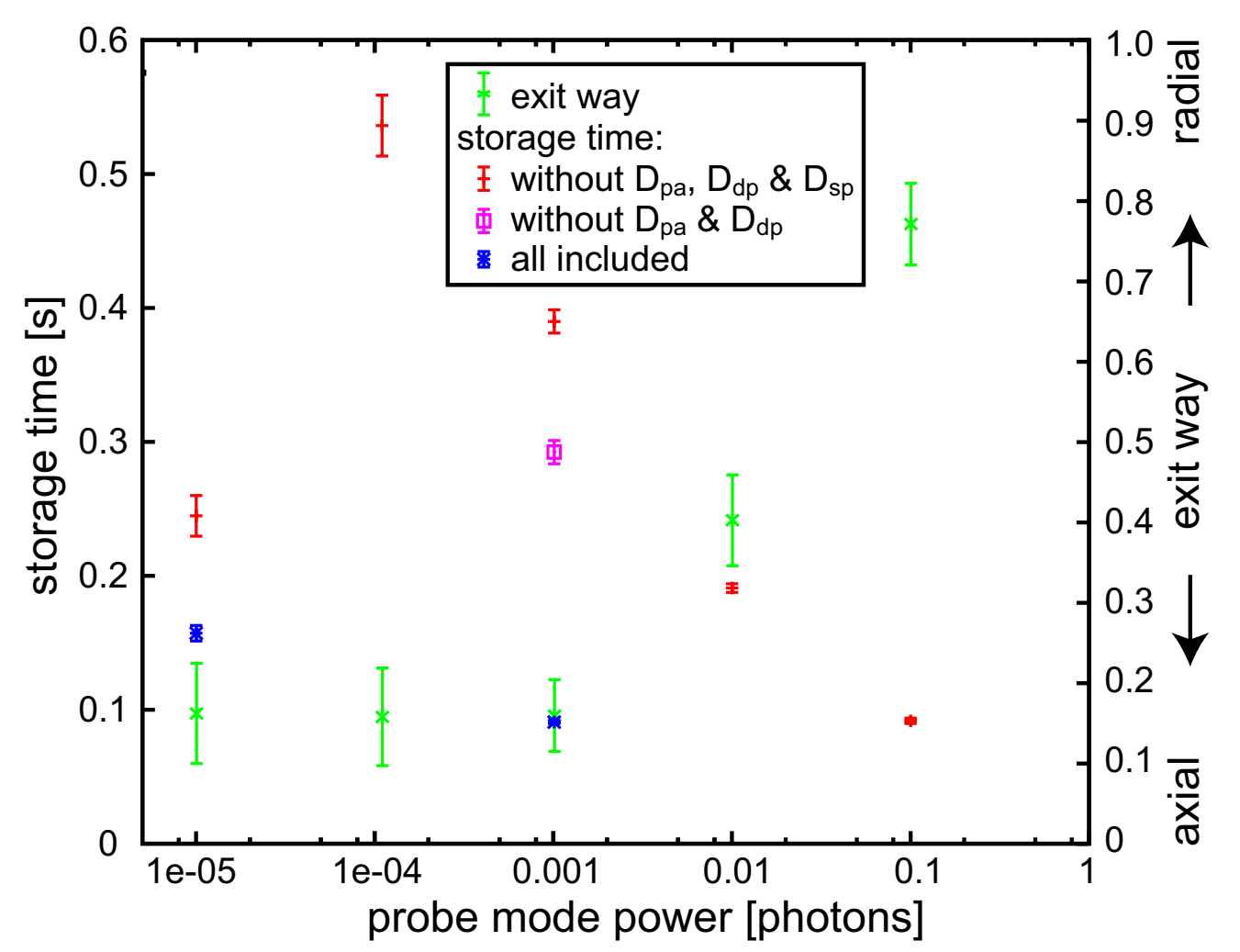

Figure 5.8: Cavity cooling in the blue trap in cooling region II. Cavity cooling increases the storage time for low powers of the near-resonant light field. Once cavity cooling dominates, the exit way changes from axial to radial. Parameters are the same as for Fig. 5.6 with $\left\{T_{\text {trig }}=0.5\right\}$. The diffusion due to the dipole force fluctuations of the far detuned fields and due to spontaneous emission were switched off in the simulation. 

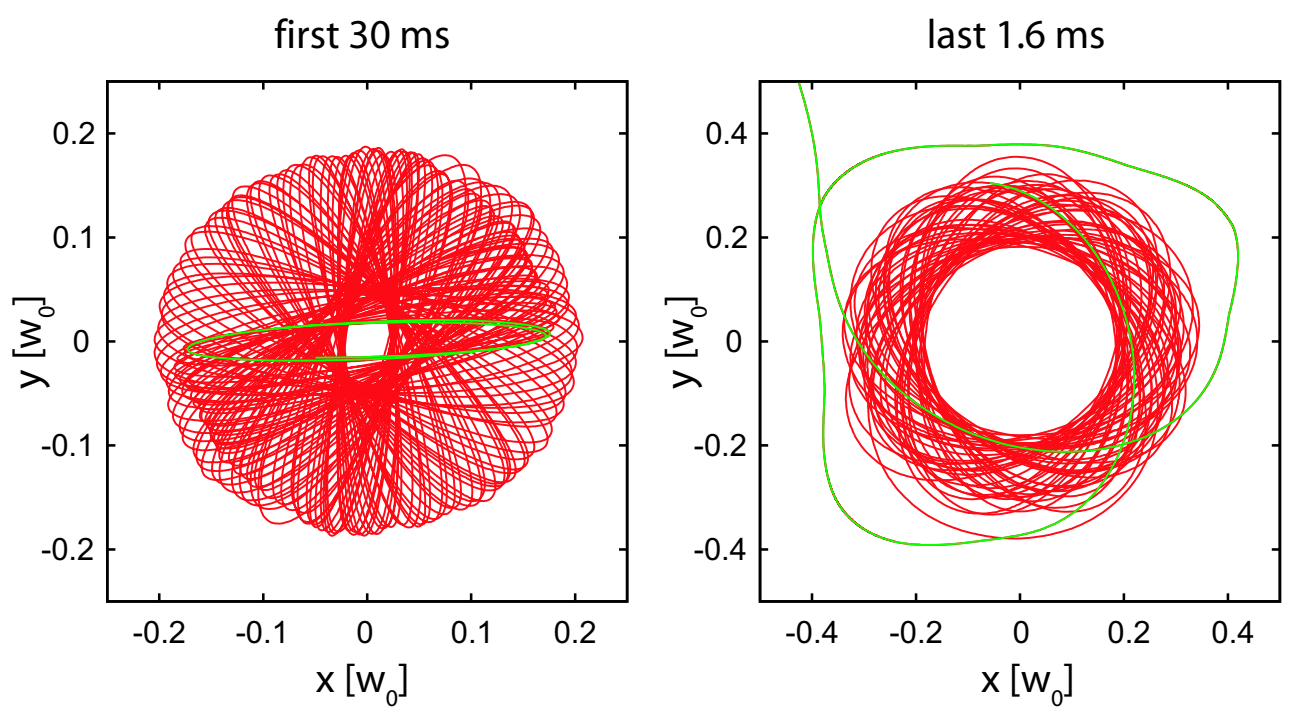

Figure 5.9: Sample trajectory: transverse motion. Simulated trajectory of an atom in the blue trap. The storage time of the atom is $529 \mathrm{~ms}$. The left plot shows the first $30 \mathrm{~ms}$ the right one the last $1.6 \mathrm{~ms}$ of the trajectory. According to the initial conditions, the trajectory in the beginning follows elliptical orbits that rotate around the symmetry center. Once the atom is able to overcome the saddle points transverse motion is distorted due to the 'hopping' along the cavity axes, see Fig. 5.10 .

$\left\{T_{\text {trig }}=0.5 ; P_{\mathrm{nr}}=1 ; x_{0}=-0.016 ; y_{0}=-0.05 ; z_{0}=-0.012 ; P_{\mathrm{r}}=0\right.$;

$\left.\left(P_{\mathrm{b} 0}, P_{\mathrm{b} 0 \mathrm{~s}}\right)=(37,37) \cdot 10^{3} ;\left(P_{\mathrm{b} 1}, P_{\mathrm{b} 1 \mathrm{~s}}\right)=(100,100) \cdot 10^{3} ;\left(\Delta_{\mathrm{a}}, \Delta_{\mathrm{c}}\right)=(-50,-6)\right\}$

\subsubsection{Sample trajectory}

To gain insight into the dynamics of the system for a typical trapping event a sample trajectory is shown in Fig. 5.9. The atom is positioned in the center antinode of the cavity. In the beginning the motion of the atom is defined by the starting conditions. The atom moves in an elliptic orbit which turns around the symmetry axis. Towards the end of the trajectory the orbit is nearly circular while the angular momentum only slightly increased. The potential landscape of the blue trap is depicted in Fig. 3.2. A saddle point is formed between the on axis maxima of the $\mathrm{TEM}_{00}$ and the shifted off-axis ring type maximum of the doughnut $\left(\mathrm{TEM}_{10}+\mathrm{TEM}_{01}\right)$ mode at approximately $\left(z=0.35 \lambda_{n r}, r=\right.$ $\left.0.43 w_{0}\right)$. The potential height at the saddle point is about $70 \%$ of the maximum value when the maxima have equal heights. Since, the saddle is strongly elongated in the radial direction it will be traced for every radial excursion, due to the much faster axial oscillation. The necessity to overcome the elongated saddle corresponds to a well defined energy filter. This shows up as a narrow axial energy distribution $(5.4 \pm 0.3 \mathrm{MHz})$ of the atom leaving the trap with a saddle point of $5.4 \mathrm{MHz}$. In contrast, the average radial energy is lower and has a much broader distribution.

At the very end of the trapping event, the trajectory shows distortions from a regular orbit. These 'bumps' coincide with flights along the standing wave direction, where the 


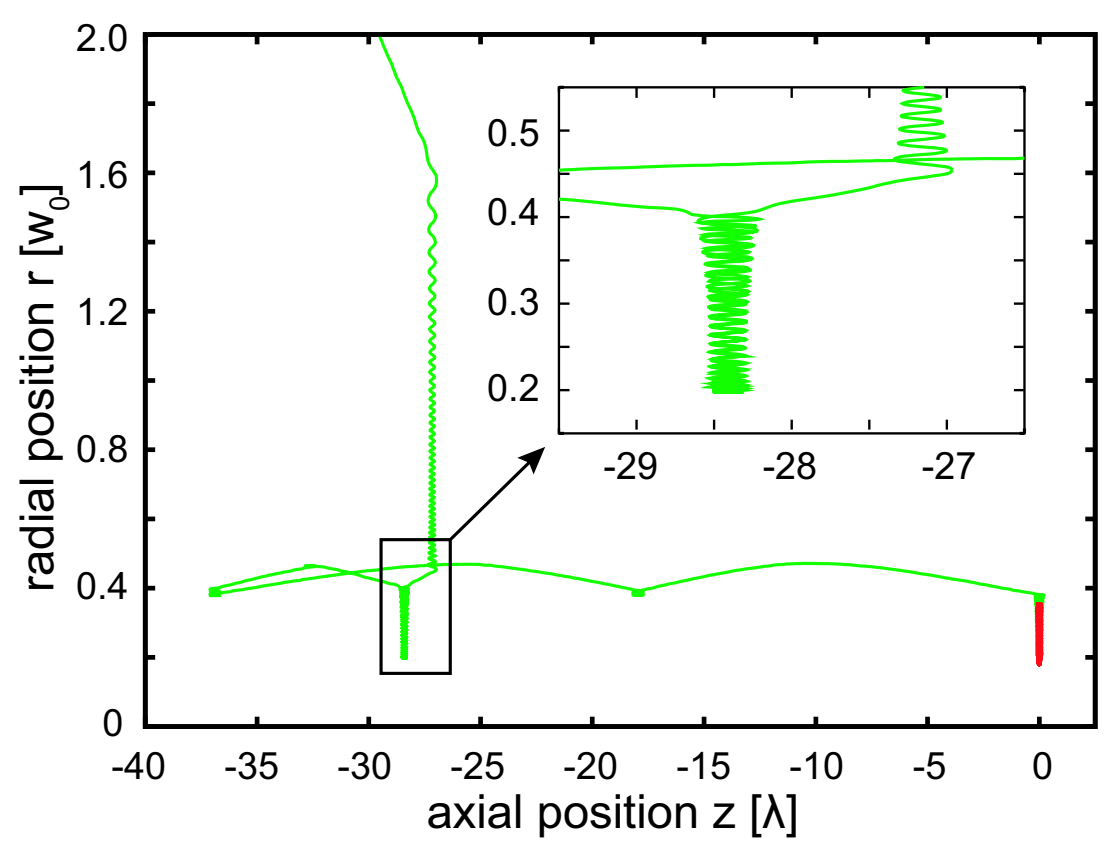

Figure 5.10: Sample trajectory: axial motion. Towards the end of the trajectory the atom has gained enough energy to overcome the saddle points. The atom then hops between different potential wells a few times. Finally, it leaves the trap by running down the outer slopes of the $\mathrm{TEM}_{00}$ mode.

atom changes between antinodes some wavelength apart (Fig. 5.10). These flights are possible once the atom can overcome the saddles. For a few more radial oscillations the atom will pass through some other trap well. Finally, it will run down one of the outer channels formed by the slopes of the TEM 00 mode and the maxima of the doughnut. This is the reason why in the blue trap the exit ways are not as well distinguishable and tend to be radial.

The close to circular orbit is a disadvantage, because efficient cooling is located close to the trap center. This problem can be solved by applying transverse cooling with extra laser beams from the side. Alternatively, higher order modes could be used to break the cylindrical symmetry and allow for azimuthal cooling.

The numerical simulations presented in this chapter confirm the properties and advantages of the blue trap projected in the discussion in Sec. 3.3 , including improved localization at the trigger and decrease in energy gain while guiding and switching. Further conclusions and prospects are discussed in Sec. 7.10 after the experimental realization of the blue trap is presented. 



\section{Chapter 6}

\section{Experimental setup}

The experiment is designed to prepare single atoms strongly coupled to the mode of a high-finesse resonator. Slow atoms are delivered from a magneto-optical trap (MOT) by means of an atomic fountain. The launch velocity is chosen such that the turning point of the ballistic trajectory is close to the position of the cavity mode. The atomic fountain is well suited to deliver atoms to small volume Fabry-Perot cavies in the strong-coupling regime. Here, optical access from the side is limited and hence orthogonal dipole traps to deterministically transport atoms to the mode (39; 118) are challenging (119). Instead, an intracavity dipole trap is used to store the atom.

In the cavity, single atoms are guided to regions of strong coupling by the dipole force of additional far-detuned cavity modes. They are detected in the cavity by their strong influence on the transmission of a near-resonant probe laser and stored by switching an intracavity dipole trap upon detection. For properly chosen parameters, the strong velocity-dependent forces cool the atom in the axial direction. At the same time, the coupling of the trapped atom to the cavity mode can be continuously observed via the cavity transmission. This enables measurements on a qualified strongly-coupled atomcavity system, like the spectroscopy presented in Chap. 8 .

The different subsystems of the experimental setup are presented in the subsequent sections. Some of them are explained in detail in previous work. The emphasis is therefore laid on the various modifications and extensions that are implemented to realize the blue intracavity dipole trap. The magneto-optical trap (120), vacuum system and atomic fountain (121) are introduced in Sec. 6.1. On their way to the high-finesse cavity (122) (Sec. 6.4), the atoms pass several laser beams used for characterization and preparation (111) (Sec.6.2 and Sec.6.3). The laser system (Sec.6.5), cavity stabilization (106) (Sec. 6.5) and detection (Sec. 6.7) require major modification compared to the red dipole trap (123; 87). Finally, systematic measurements and analysis are based on the computer controlled data acquisition presented in Sec. 6.8 . 


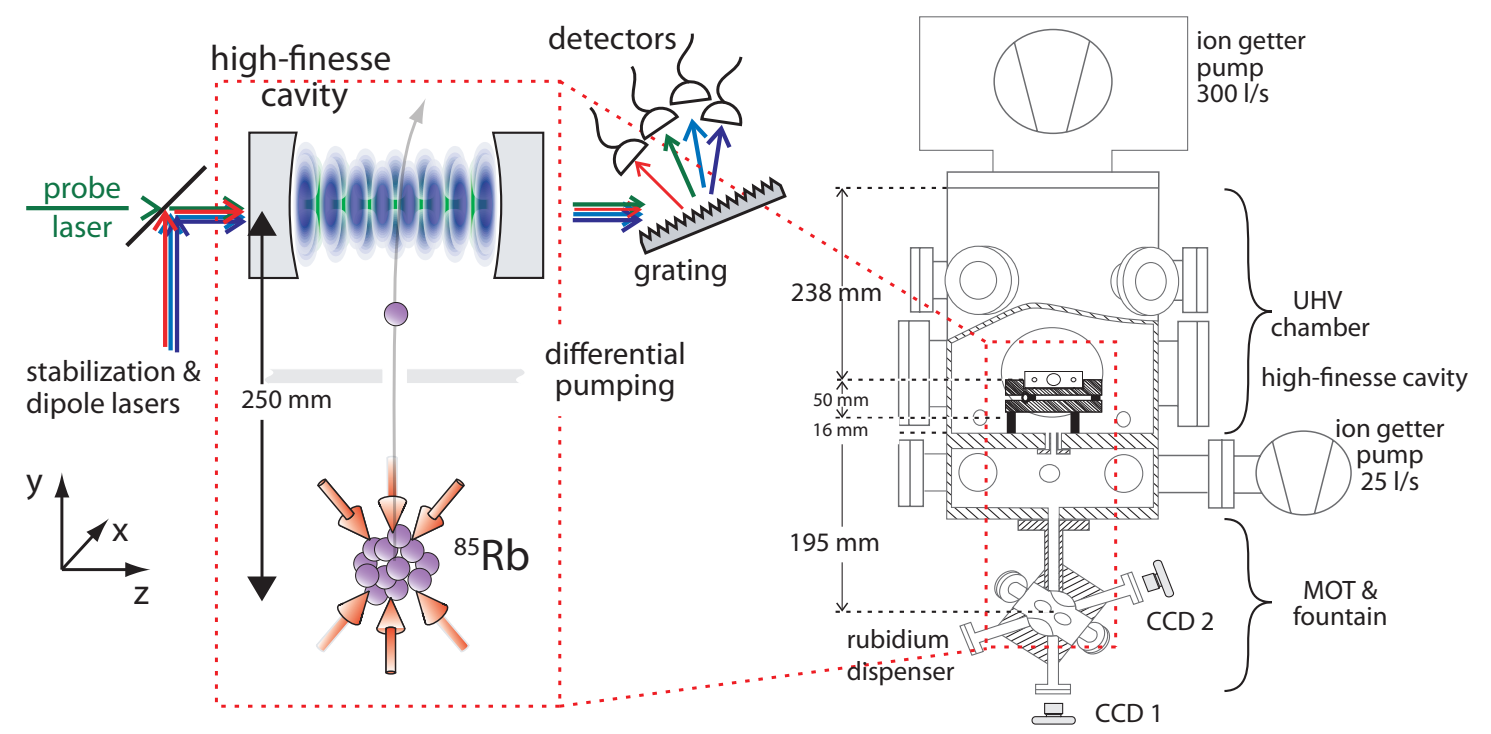

Figure 6.1: Experimental apparatus: Left: Schematic view of the atomic fountain and the high-finesse cavity with the different on-axis lasers. Right: Sketch of the vacuum chamber. The MOT cube is hanging from the lower part of the vacuum chamber. The upper (UHV) part, where the cavity is situated, is separated by a differential pumping tube.

\subsection{Magneto-optical trap and atomic fountain}

The magneto-optical trap (MOT) is located in a cube connected to the lower end of the main vacuum chamber by a differential pumping tube (Fig. 6.1, right). The space diagonal of the cube is oriented in the vertical. Six circularly-polarized laser beams are incident on the faces of the cube. They divide into two sets of three lower and three upper trapping beams. The repumper is superposed with the lower trapping beams. In the MOT $10^{7}{ }^{85} \mathrm{Rb}$ atoms are trapped from the background pressure, which is enhanced by a pulsed dispenser (SAES Getter) during the loading phase. They are cooled to temperatures of $\simeq 5 \mu \mathrm{K}$ in an optical molasses. The atoms are accelerated upward by continuously detuning the lower beams to the blue by $\simeq 1 \mathrm{MHz} / \mathrm{ms}$. Thereby, the atoms are adiabatically transferred and cooled into a moving molasses. In the moving frame, the detuning of upper and lower trap beams is exactly compensated by the Doppler-shift. The maximum detuning at the end of the acceleration phase defines the launch velocity of $\simeq 0.7 \mathrm{~m} / \mathrm{s}$. The atomic fountain conveys the atoms through the differential pumping tube (stainless steel, $2 \mathrm{~mm} \times 20 \mathrm{~mm}$ ) into the main vacuum chamber. The pressure in the main chamber is a few times $10^{-10}$ mbar such that collisions with background gas can be neglected. The final velocities of the atoms at the position of the cavity mode can be chosen by the detuning of the lower beams from $0.1 \mathrm{~m} / \mathrm{s}$ to a few $\mathrm{m} / \mathrm{s}$. A further selection on the arriving times of the late tail of the atom cloud guarantees velocities $v<0.1 \mathrm{~m} / \mathrm{s}$.

The relevant transition for the MOT and the atomic fountain are shown in Fig. 6.2. 


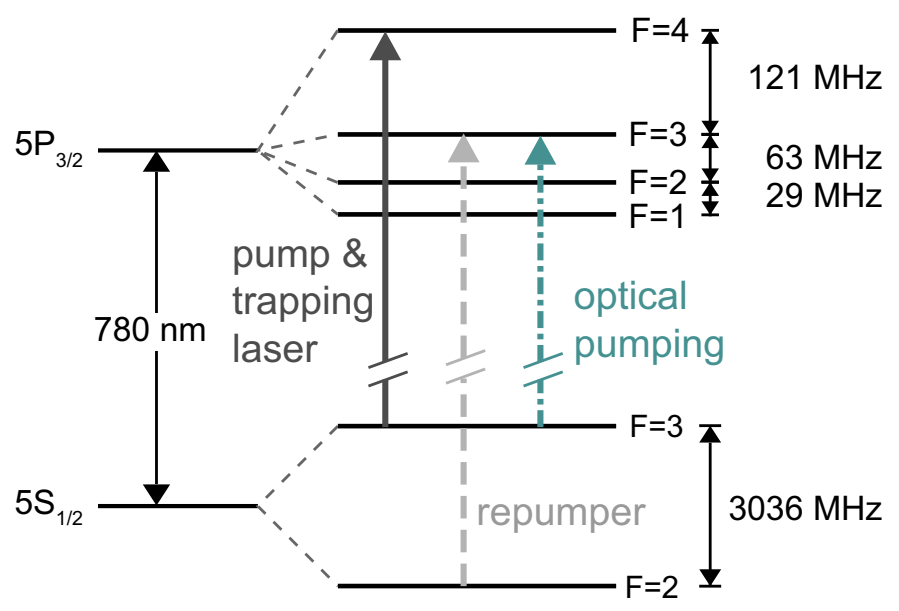

Figure 6.2: Level scheme of ${ }^{85} \mathbf{R b}$ for the relevant transitions between the $5 S_{1 / 2}$ and $5 P_{3 / 2}$ states. The probe mode is close to the $5 S_{1 / 2}, \mathrm{~F}=3 \rightarrow 5 P_{3 / 2}, \mathrm{~F}=4$ cycling transition also used for trapping in the MOT. The repumper returns atoms from the $5 S_{1 / 2}, F=2$ state back into the cycling transition. See appendix A for the level scheme including higher states.

To cool the atoms into a moving frame in the atomic fountain, the MOT beams can be independently red detuned in the range of 0 to $40 \mathrm{MHz}$ with respect to the trap transition $5 S_{1 / 2}, \mathrm{~F}=3 \rightarrow 5 P_{3 / 2}, \mathrm{~F}=4$ (Fig. 6.2). With a small residual probability, the atoms are non-resonantly excited to the $5 P_{3 / 2}, \mathrm{~F}=3$ state. From this state they can decay to the lower ground state $5 S_{1 / 2}, \mathrm{~F}=2$. The repumper excites these atoms to the state $5 P_{3 / 2}, \mathrm{~F}=3$, from which they are eventually transferred back to the upper ground state and the cycling transition. Because of the large detuning with respect to the pump laser of $2915 \mathrm{MHz}$, the repumper is directly stabilized on the repumping transition by Doppler-free saturation spectroscopy. See Fig. 6.4 for an overview of the laser system. To increase the reliability all trapping beams pass through polarization maintaining single-mode fibers (PMSMF) and are intensity stabilized. An additional, more powerful external-cavity diode laser (ECDL; DL100, Toptica) in Littrow configuration has been installed as a new repumper and delivers about five times more intensity-stabilized power in an improved spatial mode.

On their way to the cavity the atoms optionally pass the fluorescence beam used for alignment and characterization and the optical pumping beam used to define the magnetic field at the cavity and for preparation of the internal atomic state.

\subsection{Fluorescence laser}

The fluorescence beam is used for the alignment of the atomic fountain. It is derived from light of the lower MOT beams containing repumper light and crosses the upper UHV chamber below the cavity. The fluorescence signal measured with a photomultiplier tube (PMT) is proportional to the number of atoms that reach the upper vacuum chamber. 
To prevent the atoms from deflection by unbalanced recoils the fluorescence beam is retroreflected. If the beam is not retroreflected, it can also be used to cut into the atom distribution of the cloud.

\subsection{Optical pumping beam}

The optical pumping beam can be used to pump the atoms into the desired Zeeman sublevel just before entering into the cavity. The pumping beam is resonant with the $5 S_{1 / 2}, \mathrm{~F}=3 \rightarrow 5 P_{3 / 2}, \mathrm{~F}=3$ transition and contains some repumping light. In a magnetic field collinear with the cavity axis and the direction of the $\sigma^{+}$-polarized beam, the atoms are pumped into the $5 S_{1 / 2}, \mathrm{~F}=3, m_{F}=3$ dark state. The beam is also retroreflected to prevent deflection of the atoms. In the experiments described later, this beam is not necessary, because optical pumping is achieved by the intracavity probe field.

Inside the cavity the quantization axis is given by a residual magnetic field aligned along the cavity axis. The magnetic field in the cavity is defined by switching the coils used to compensate the earth magnetic field at the position of the MOT to a predefined value. The existence of the dark state can be used to compensate the orthogonal field components. If the magnetic field is well aligned in the cavity, the limited number of recoils transferred to the atoms by an unbalanced beam will not lead to a large deflection sufficient to prevent detection of the atoms in the cavity (106).

\subsection{High-finesse cavity}

The science cavity (Fig. 6.3) is a Fabry-Perot resonator. The field in the cavity can be treated in the paraxial approximation given by an axial standing wave and a transverse mode pattern. The cavity length $l=156 \times \lambda_{\mathrm{nr}}=122 \mu \mathrm{m}$ is calculated from the freespectral rage measured as half the frequency difference of two resonant lasers (TiSa780, ECDL785) (i.) to $\Delta \nu_{\mathrm{FSR}}=2 \pi \times 1.23 \mathrm{THz}$. Because the length is much smaller than the radii of curvature $R=200 \mathrm{~mm}$ of the two mirrors, the science cavity is near-planar. In the near-planar limit $l / R \ll 1$ the Rayleigh length $z_{r}$ is much larger than the length

\footnotetext{
(i.) The abbreviations for the different lasers are constructed from the type and the wavelength. TiSa denotes a diode-pumped solid-state (DPSS) pumped titanium saphire ring laser system (MBR-110 Monolithic Block Resonator pumped by a Verdi V10, Coherent). ECDL stands for external-cavity diode laser in Littrow configuration (mostly DL 100 (XL), Toptica, the old repumper and trapping lasers are homebuild).
} 
$\left(l / z_{r} \ll 1\right)$ and the cavity parameters can be further simplified:

$$
\begin{aligned}
z_{r} & =\frac{l}{2} \sqrt{\frac{2 R}{l}-1} \approx \sqrt{\frac{R l}{2}} \\
w_{0} & =\sqrt{\frac{\lambda}{\pi} z_{r}} \approx \sqrt{\frac{\lambda}{\pi}} \sqrt[4]{\frac{R l}{2}} \\
w(z) & \approx w_{0}\left(1+\frac{1}{2}\left(\frac{z}{z_{r}}\right)^{2}\right)^{1 / 2} \approx w_{0}
\end{aligned}
$$

where $z$ is the coordinate along the cavity axis.

The waist of the near-resonant mode is given by the wave length $\lambda_{\mathrm{nr}}=780.2 \mathrm{~nm}$, the cavity length and the mirror curvature (124). In the near-planar limit the waist does not change much within the mirrors, cf. eqn. 6.3). The coupling between the atom and the field is given by:

$$
g=\sqrt{\frac{\omega_{c}}{2 \epsilon_{0} \hbar V}} d_{g e}=2 \pi \times 16 \mathrm{MHz}
$$

Here, $d_{g e}$ is the dipole matrix element of the transition $\left(5 S_{1 / 2}, F=3, m_{F}=3 \rightarrow\right.$ $\left.5 P_{3 / 2}, F=4, m_{F}=4\right)$ and $\epsilon_{0}$ is the vacuum permittivity. The small mode volume $V$ leads to a large dipole coupling $g \propto V^{-1 / 2}$. The cavity field decay rate of $\kappa=$ $2 \pi \times 1.4 \mathrm{MHz}$ is measured by observing the exponential decay of the cavity transmission. With the free-spectral range (FSR) of $\Delta \nu_{\mathrm{FSR}}=1.23 \mathrm{THz}$, the finesse of the cavity is evaluated to $\mathcal{F}=4.4 \cdot 10^{5}$. The transmission $(2.8 \mathrm{ppm})$ and losses $(7.3 \mathrm{ppm})$ of each mirror are characterized by the simultaneous measurement of reflection and transmission

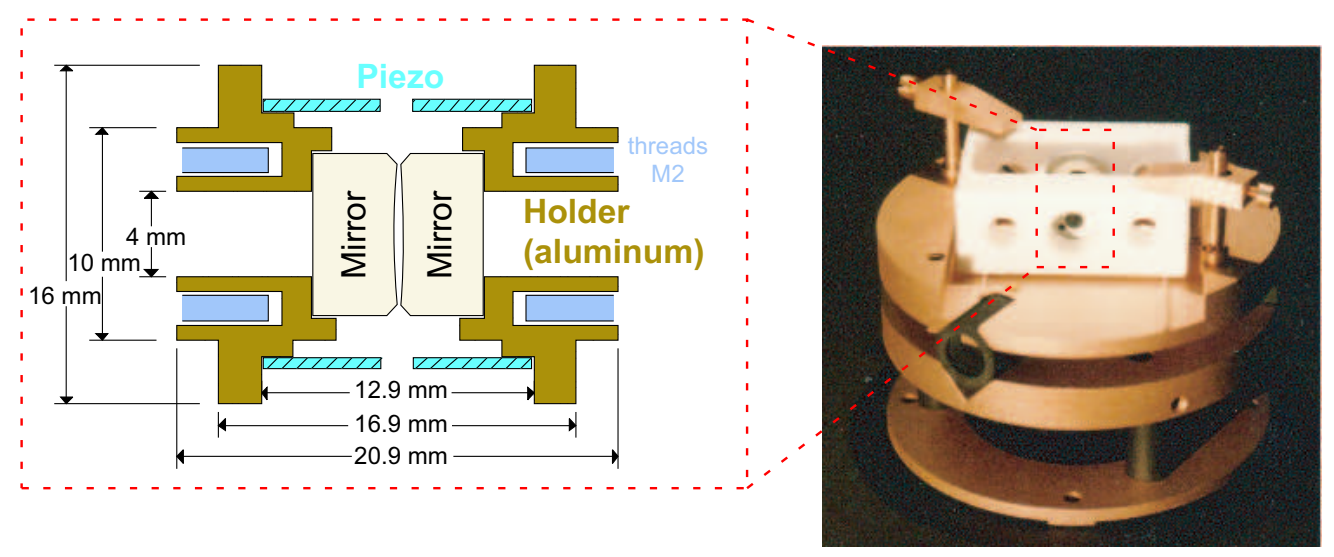

Figure 6.3: High-finesse cavity. Left: technical drawing of the cavity setup. The mirrors are glued stress-free to aluminum holders, which are tight-fit into the piezo tube. Right: photograph of the complete cavity setup including the Teflon bracket and the vibration isolation stage with Viton cylinders. 


\begin{tabular}{|c|c|c|}
\hline Parameter & Value & Comment \\
\hline radius of curvature & $R=200 \mathrm{~mm}$ & \\
\hline mirror diameter & $d=7.75 \mathrm{~mm}$ & \\
\hline mirror transmission & $T=2.8 \mathrm{ppm}$ & \\
\hline mirror losses & $L=7.3 \mathrm{ppm}$ & \\
\hline length & $l=122 \mu \mathrm{m}$ & from $\Delta \nu_{\mathrm{FSR}}=\frac{c}{2 l}$ \\
\hline free spectral range & $\Delta \nu_{\mathrm{FSR}}=1.28 \mathrm{THz}$ & \\
\hline linewidth & $\Delta \nu_{\mathrm{FWHM}}=2.8 \mathrm{MHz}$ & \\
\hline field decay rate & $\kappa=2 \pi \times 1.4 \mathrm{MHz}$ & \\
\hline finesse & $\mathcal{F}=4.4 \times 10^{5}$ & $\mathcal{F}=\frac{\Delta \nu_{\mathrm{FSR}}}{\Delta \nu_{\mathrm{FWHM}}}$ \\
\hline mode waist & $w_{0}=29.1 \mu \mathrm{m}$ & \\
\hline coupling constant & $g_{0}=2 \pi \times 16 \mathrm{MHz}$ & $g_{0}=\sqrt{\frac{\omega_{c}}{2 \epsilon_{0} V \hbar}} d_{g e}$ \\
\hline$P_{\text {trans }}$ for $\left\langle a^{\dagger} a\right\rangle=1$ (iii.) & $0.86 \mathrm{pW}$ & \\
\hline \multicolumn{2}{|c}{}
\end{tabular}

Table 6.1: Parameters of the high-finesse cavity.

under the assumption of two identical mirrors (125). An alternative method to measure the field decay time is to scan across the resonance and observe a frequency beat of the Doppler-shifted circulating field on top of an exponential decay (126). The cavity parameters are summarized in table 6.1.

The dielectric mirrors are glued stress-free to aluminum holders which are tight-fit into both sides of a piezo-ceramic tube(ii.) . This setup is compressed by the teflon frame as shown in Fig. 6.3. By applying a high voltage to the piezo the cavity length can be varied by several $100 \mathrm{~nm}$. The cavity does not show a significant splitting in the transmission of $\sigma^{+}$- and $\sigma^{-}$- circularly polarized light, i.e. the observed birefringence is within the linewidth. An analysis of the polarization dependence on the modes of order one is given in appendix $\mathrm{C}$. The cavity length is stabilized independently of the probe with a Pound-Drever-Hall stabilization in reflection (Sec. 6.6).

\subsection{Laser system}

A functional overview of the laser system is shown in Fig. 6.4. All near-resonant light beams are derived from the main laser (TiSa780) by double-pass acousto-optical modu-

\footnotetext{
${ }^{(i i .)}$ Within the framework of this thesis a new science cavity design has been developed together with Mr. Bayerl. The coned mirrors are now tight-fitted into a 'crown' of springs, machined from solid aluminum. These holders are UHV compatible and do not seem to induce any extra birefringence. With supplemental tools, they allow to exchange the mirrors during the setup and characterization of the cavity. They are incorporated in the new cavity setups using coned mirrors

(iii.) Transmitted power for an mean photon number of one in the cavity, i.e. $\left(\left\langle a^{\dagger} a\right\rangle \equiv\right.$ 1) $2 \kappa 1 / 2 \frac{T}{L+T} E_{\text {Photon, }}$, where $2 \kappa$ is the decay rate of the field. Assumed the mirrors are identical, half of the $\frac{T}{L+T} \approx 0.183$ the photons with energy $E_{\text {Photon }} \approx 2.51 E-19 \mathrm{~J}$ are emitted through one of the mirrors which corresponds to a power of $0.86 \mathrm{pW} . T, L$ are the transmission and losses per mirror
} 


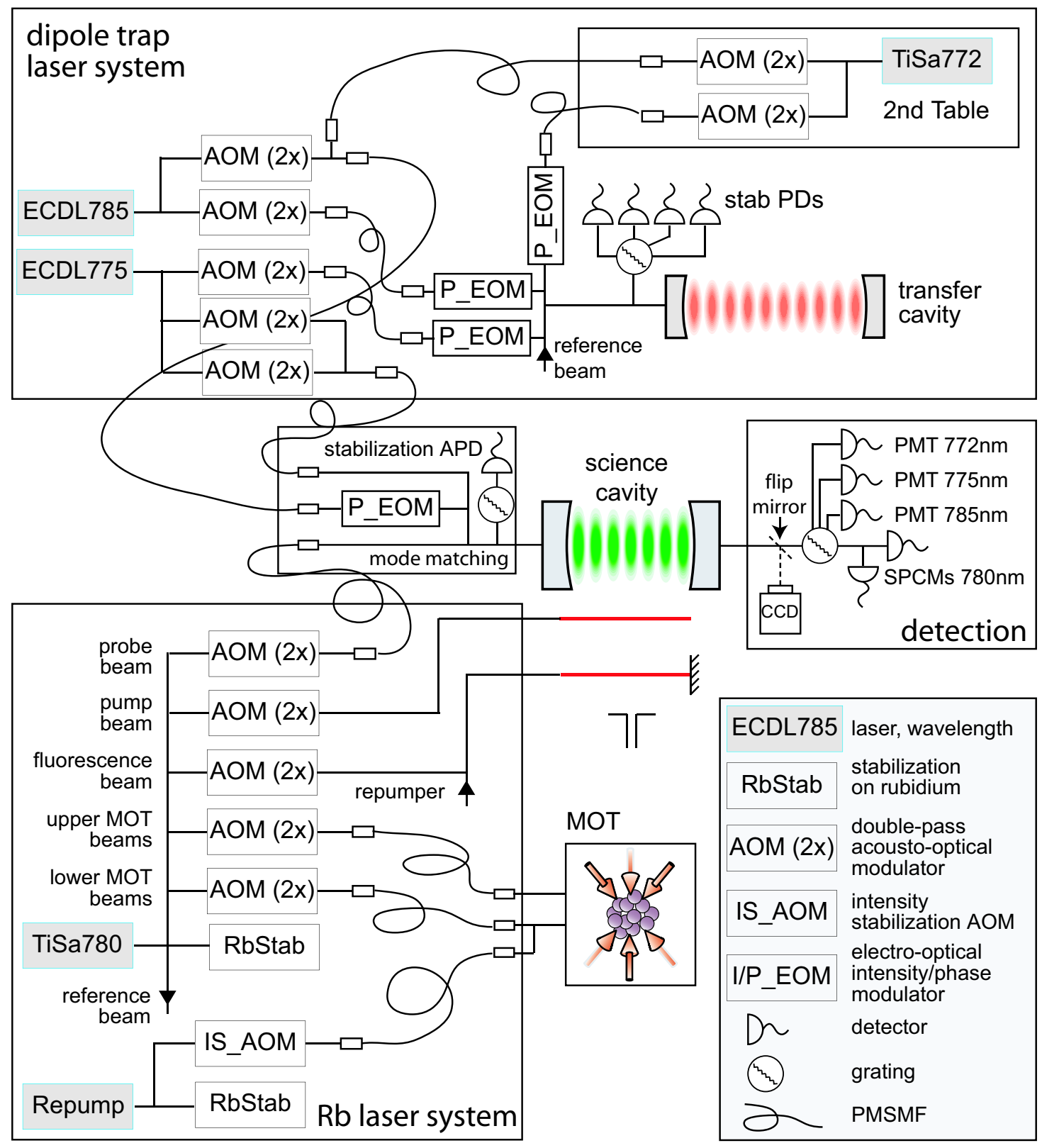

Figure 6.4: Overview of the laser system: Laser beams are derived from five different lasers for different applications in the experiment. Acousto-optical modulators (AOMs) allow to independently adjust the frequencies and stabilize the intensities behind the polarization maintaining single-mode fibres (PMSMF). The main TiSa780 and the repumper are locked to rubidium, all far-detuned dipole lasers are locked to the transfer cavity. Electro-optical phase modulators are used for the Pound-Drever-Hall locks. A total of five different beams delivered through three fibers pump the cavity on axis. For the detection the beams are separated by a grating and directed to different detectors. 
lators (AOM). The beams for the magneto-optical trap (MOT) are delivered by polarization maintaining single-mode fibres (PMSMF). The polarization-cleaned output of all fibre-based beams is intensity stabilized by feedback onto the radio-frequency (rf) power of the AOMs. Both the TiSa780 as well as the repumper are stabilized by Doppler-free saturation spectroscopy. All far-detuned dipole lasers (ECDL785, ECDL775, TiSa772) are stabilized in reflection by a Pound-Drever-Hall lock onto the transfer cavity, which itself is stabilized to the TiSa780. Five beams are delivered to the cavity by three fibres (PMSMF) and mode-matched to the science cavity. In transmission, the different major beams are split by wavelength and directed to different detectors. The mode matching of the on-axis beams, the cavity stabilization and the detection will be discussed in more detail below.

\subsubsection{Dipole laser stabilization}

The general concept of the laser stabilization of the far-detuned dipole laser is illustrated in Fig. 6.5. Two beams are individually frequency shifted by double-pass AOM setups and mode cleaned by polarization maintaining single-mode fibres. One is sent to the science cavity, the other to the transfer cavity.

The transfer cavity is a near-planar Fabry-Perot cavity (127). It has a length of $470 \mathrm{~mm}$, a free-spectral range of $310 \mathrm{MHz}$ and a full-width half-maximum (FWHM) linewidth of $100 \mathrm{kHz}$. Owing to the ultralow expansion (ULE) spacers, vibration isolation and mounting in a vacuum tube the transfer cavity is mechanically very stable with a temperature induced frequency drift of $\sim 100 \mathrm{kHz} / \mathrm{s}$. On timescales larger than $1 \mathrm{~s}$ the transfer cavity is stabilized to the main laser (TiSa780) with a low pass defined bandwidth of about $100 \mathrm{~Hz}$.

To realize the Pound-Drever-Hall lock (128), sidebands are modulated onto the dipole laser beam by an electro-optical phase modulator (P_EOM). The rf-frequency of about $20 \mathrm{MHz}$ is much larger than the linewidth of the transfer cavity. It allows for a high bandwidth $(4 \mathrm{MHz})$ of the error signal and determines the capture range. The error signal, obtained by mixing the signal of the photo diode with the local oscillator with the appropriate phase, shows a steep slope in the range of the transfer cavity linewidth. The error signal is fed back by three branches of decreasing non overlapping frequency ranges (similar to (129)): (1) Directly to the laser diode current (bias-T), (2) with a servo (proportional plus integral plus derivative controller, PID) to the laser current ( $100 \mathrm{kHz}$ bandwidth, forgetting integrator), (3) via a PID to the piezo tilt of the external cavity grating. An amplifier picks up the signal for the two low bandwidth feedbacks from the $50 \mathrm{Ohm}$ line to the bias-T.

Both frequency locks of the external cavity diode lasers (Toptica DL100 (XL), Littrow configuration) share the same local oscillator and EOM producing $19 \mathrm{MHz}$ sidebands. Their linewidths are reduced from about $1 \mathrm{MHz}$ to $\lesssim 20 \mathrm{kHz}$ (r.m.s., root mean square). The noise power at low frequencies is strongly suppressed, the residual noise power spectrum peaks at about $1 \mathrm{MHz}$. The linewidth of the TiSa772 is also about $20 \mathrm{kHz}$ (r.m.s.) using sidebands with $17 \mathrm{MHz}$ modulation frequency. To allow for independent stabilizations, the laser beams are separated by a holographic grating (Edmund Scientific, 
2400 lines/mm, $\sim 50 \%$ efficiency) and directed to individual rf photodiodes.

\subsubsection{On-axis cavity excitation}

All beams that are coupled to the science cavity on axis through one of the mirrors are mode cleaned by a polarization maintaining single-mode fiber (PMSMF). The intensity is stabilized behind an additional, high extinction-ratio $\left(>10^{-6}\right)$ linear polarizer (Polacor, Corning) by varying the rf-intensity on the AOM. The beams are coupled to the corresponding cavity modes using appropriate telescopes and periscopes and are superimposed by an arrangement of beam splitters. Since they all pass the final polarizing beamsplitter (PBS) and quarter-wave plate (QWP), they are circularly polarized when reaching the cavity. The reflected beams leave the PBS through the other port. The far-detuned beams are frequency shifted by a multiple of the free-spectral range of the

\begin{tabular}{|c|c|c|c|}
\hline كى & $\begin{array}{l}\text { external cavity } \\
\text { diode laser }\end{array}$ & D detector & \\
\hline $\operatorname{AOM}(2 x)$ & $\begin{array}{l}\text { double-pass } \\
\text { acousto-optical }\end{array}$ & $\begin{array}{l}\text { ( source } \\
\tau \text { delay }\end{array}$ & $\begin{array}{l}\text { polarizing beam } \\
\text { splitter }\end{array}$ \\
\hline P_EOM & $\begin{array}{l}\text { electro-optical phase } \\
\text { modulator }\end{array}$ & $\sum_{(M x)} a$ & $\begin{array}{l}\text { wave plate } \\
\text { nonpolarizing } \\
\text { beam splitter }\end{array}$ \\
\hline
\end{tabular}

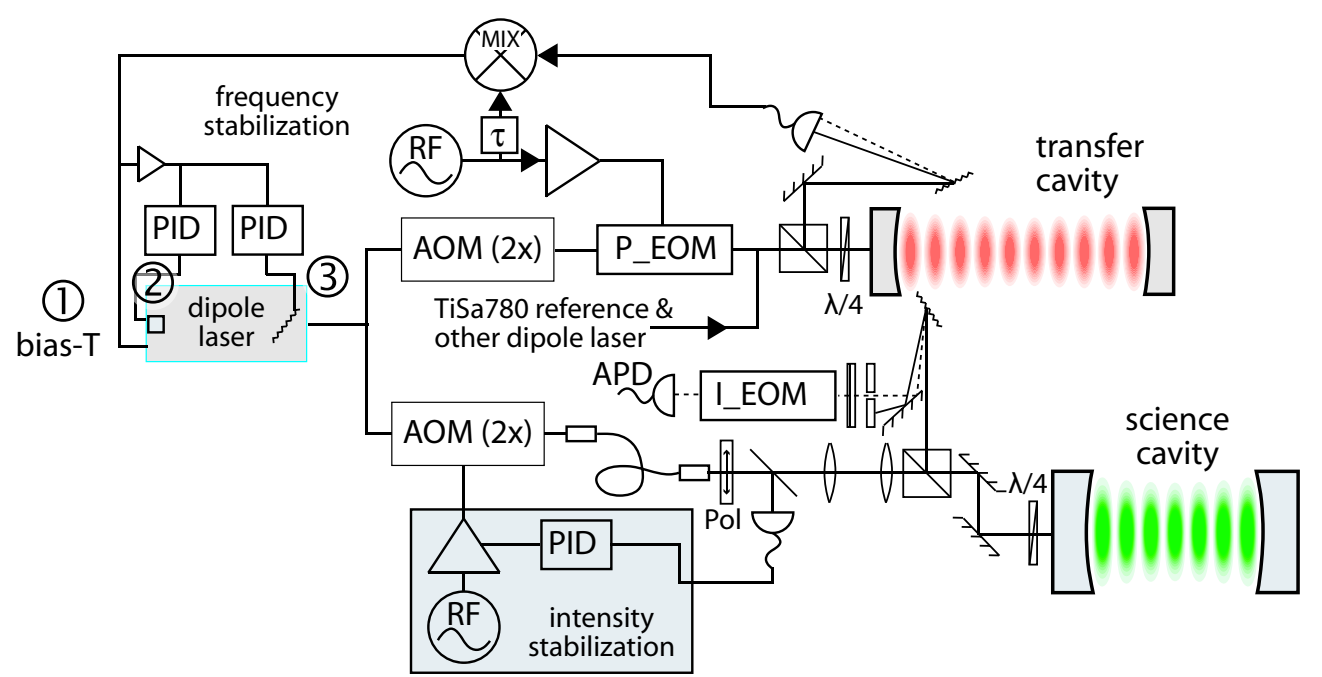

Figure 6.5: Stabilization of the far-detuned dipole lasers is realized by a Pound-DreverHall lock to the transfer cavity which itself is stabilized to the main TiSa780 laser. The error signal is obtained in reflection by radio-frequency ( $\mathrm{rf}$ ) phase modulation with an electro-optical modulator and mixing the photo diode output with the local oscillator. The error signal is directly fed to the laser diode via a Bias-T (1). A lower bandwidth error signal from a pickup amplifier is provided to two PID servos. The correction signals with decreasing non overlapping frequency rages are applied to the current (2) and the grating (3). The linewidths of the dipole lasers are about $20 \mathrm{kHz}$. 
science-cavity which corresponds to a detuning of $\simeq 2.5 \mathrm{~nm}$. The beam used for stabilization is split from the others by a holographic grating (Edmund Scientific) and directed to an avalanche photo diode (APD) (see Sec. 6.6 for details).

\subsubsection{Probe beam $(780.24 \mathrm{~nm})$}

The weak probe beam with an intensity on the order of one $\mathrm{pW}$ can be shifted by $\simeq$ $\pm 50 \mathrm{MHz}$ with respect to the $5 S_{1 / 2}, \mathrm{~F}=3 \rightarrow 5 P_{3 / 2}, \mathrm{~F}=4$ transition. In most experiments it is coupled to the fundamental $\mathrm{TEM}_{00}$ cavity mode.

\subsubsection{Red trap and stabilization laser (785.2 nm)}

The stabilization of the red dipole trap laser (Toptica DL100 XL) was improved by implementing a specifically designed pick-up amplifier to reduce the phase shift in the feedback loop. In most of the experiments discussed in Chap. 7, the red dipole laser is used for the stabilization of the cavity. In the measurement of the anti-crossing of a single trapped atom strongly coupled to the mode presented in Sec. 8.2 it served as a red-detuned intracavity dipole trap.

The blue trap consists of two more laser beams (for details on the geometry of the trap see 7.1):

\subsubsection{Axial confinement laser: pancakes $(772.5 \mathrm{~nm})$}

The laser beam pumping the $\mathrm{TEM}_{00}$ mode used for axial confinement is delivered from the TiSa772 to the cavity via the same fibre (PMSM) as the stabilization laser(ECDL785). For intensity stabilization the two beams are split by a holographic grating (Edmund Scientific) and are directed to two photo diodes. It excites a TEM 00 cavity mode to form a one-dimensional array of oblate repellent field antinodes, i.e a 'stack of pancakes'.

\subsubsection{Transverse guiding \& trapping: funnels \& doughnut $(775.2 \mathrm{~nm})$}

The laser (ECDL775) for transverse guiding and trapping actually consists of two beams that can be independently detuned in frequencies by acousto-optical modulators. For guiding, only one of the beams is exciting a superposition of the $\mathrm{TEM}_{10}$ and $\mathrm{TEM}_{01}$ eigenmodes, which are oriented at $\pm 45^{\circ}$ with respect to the vertical. This superposition corresponds to a $\mathrm{TEM}_{10}$ mode, restricting the atoms to its nodal line oriented in the vertical direction. To trap the atoms upon detection in the near-resonant cavity mode, both eigenmodes are excited on resonance to from a doughnut shaped ring for full confinement, see Fig. 7.2 for details. The higher-order modes are excited by the mode cleaned $\mathrm{TEM}_{00}$ mode from the polarization maintaining single-mode fibre (PMSMF). It is therefore necessary to have independent control on the coupling to the science cavity and the achievable mode matching is limited. Since the mode matched intensity of the ECDL775 laser is the limitation for the attainable (transverse) trap height, its intensity was boosted by a tapered amplifier (BoosTA, Toptica). Alternatively, an adapted phase 
plate (programmable LCD) for the mode-matching would greatly improve the coupling efficiency.

\subsection{Science cavity stabilization}

The science cavity is stabilized in reflection by a Pound-Drever-Hall lock. The reflected beam used for stabilization is separated from the other on-axis beams by a grating and directed to an avalanche photo diode (APD). The ECDL785 and the TiSa772 reach the cavity via the same fibre and mode matching optics. They both pass the P_EOM that modulates rf-sidebands at $10.7 \mathrm{MHz}$ and hence, can be used alternatively for stabilization. The signal of the APD is mixed with the local oscillator to obtain the error signal. The low-voltage correction signal generated by a PID servo is fed back to the piezo to stabilize the length of science cavity. The correction signal is applied to one of the contacts floating on an additional low bandwidth, well-stabilized high-voltage offset (0 to $200 \mathrm{~V}$ ) for coarse adjustment.

If the intensity of the stabilization light is switched for trapping, the scaling of the error signal is compensated by dividing it by the impinging intensity. In addition, nonlinear contributions are counterbalanced by an electro-optical intensity modulator (I_EOM) in the path to the APD. With this compensation the intensity of the laser intensity can be switched by a factor of 10 without affecting the cavity stabilization. This is crucial for the implementation of the red intracavity dipole trap, where the ECDL785 is both stabilization and trapping laser. Note that the TiSa772 laser is particularly suited for the stabilization, because it need not be switched for the blue trap.

\subsection{Detection}

For the detection the different light beams emitted from the cavity are separated by a high-quality holographic reflection grating (American Holographics) with 2300 lines/mm and a maximum efficiency of $90 \%$ for vertically polarized light. To benefit from the maximum efficiency, the light is split into linear components with a polarizing beam splitter (PBS). The horizontal polarization is rotated by a half wave plate $(\lambda / 2)$. After reflection from the grating the originally vertically polarized beam is rotated by a half wave plate $(\lambda / 2)$ and the beams are recombined on a second PBS (see Fig. 6.7). The dipole beams are picked up by sharp-edged mirrors, which are positioned in the beam in the focal plane of a lens (L) using micro translation stages. The beams are then directed to the photo-multiplier modules (H6780-20, Hamamatsu) and the signal is amplified by a $500 \mathrm{k} \Omega$ transimpedance amplifier (Femto) with $4 \mathrm{MHz}$ bandwidth.

In the experiment, the atom-cavity-trap system is observed by detecting the transmitted probe light. Measurements are performed in the low excitation regime at very low probe intensities. One photon in the cavity mode corresponds to $0.9 \mathrm{pW}$ transmitted power or a single photon rate of $3.5 \mathrm{MHz}$. The probe light is detected by two single photon counting modules (SPCM AQR-13, Perkin \& Elmer) that allow for a high quantum efficiency of about $50 \%$ at a low dark count rate of a few $100 \mathrm{~Hz}$ and a dead time of 


\begin{tabular}{|c|c|c|c|c|}
\hline laser & dipole laser & 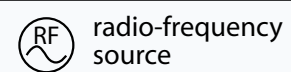 & $y^{7}$ mirror & polarizer \\
\hline $\operatorname{AOM}(2 x)$ & $\begin{array}{l}\text { double-pass } \\
\text { acousto-optical } \\
\text { modulator }\end{array}$ & $\begin{array}{l}\tau \text { delay } \\
>\text { amplifier }\end{array}$ & $\Longleftrightarrow$ lens & $\begin{array}{l}\text { polarizing beam } \\
\text { splitter }\end{array}$ \\
\hline P_EOM & $\begin{array}{l}\text { electro-optical phase } \\
\text { modulator }\end{array}$ & mixer & $D$ detector & $\begin{array}{l}\text { nonpolarizing } \\
\text { beam splitter }\end{array}$ \\
\hline
\end{tabular}

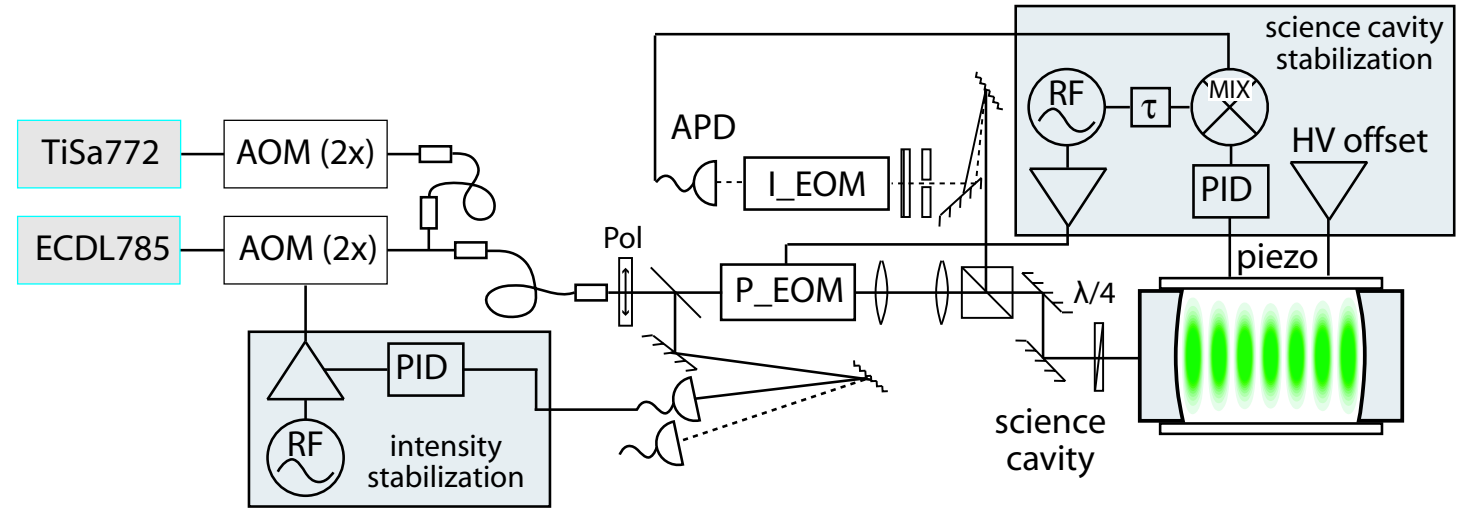

Figure 6.6: Stabilization of the high-finesse cavity. The science cavity can be alternatively locked to intensity-stabilized beams of the ECDL785 or TiSa772 laser using a Pound-Drever-Hall rf-lock at $10.7 \mathrm{MHz}$ in reflection.

$50 \mathrm{~ns}$. Because the trapping light (a few $10 \mathrm{nW}$ to $\mu \mathrm{W}$ ) is about six orders of magnitude stronger than the probe light (a few $10 \mathrm{fW}$ to $1 \mathrm{pW}$ ), special care has to be taken to reduce residual trapping light on the SPCMs. The separation by the grating reduces the dipole light by about $50 \mathrm{~dB}$. The remaining light is mainly diffuse reflection from the grating. To further reduce the dipole light by about $33 \mathrm{~dB}$, the probe light passes a narrow band interference filter (NanoLayers) with a pass band of $1 \mathrm{~nm}$ centered at $780 \mathrm{~nm}$ and a maximum transmission of $80 \%$. The overall detection efficiency for the detection of probe light transmitted by the cavity is about $30 \%$. This includes the propagation losses through the optics and the quantum efficiency of the detectors (SPCMs). The single photon clicks are registered with 1 ns time resolution (P7888, FASTComptec computer card) and stored for later analysis. A custom-made count rate to voltage converter using ECL electronics (87) generates an analog signal with an update rate of $100 \mathrm{kHz}$. A low pass filtered version of this analog signal is used for the single atom detection to trigger the trap. Alternatively to a simple level trigger a 'smart trigger' circuit triggers when the signal stays to one side of a threshold for a preselected time.

\subsection{Data acquisition}

The experiment is completely computer controlled by a system of four Linux operated computers with custom server-client software written in $\mathrm{C}++$. The system typically cycles between different measurement sequences and corresponding parameter sets. All 


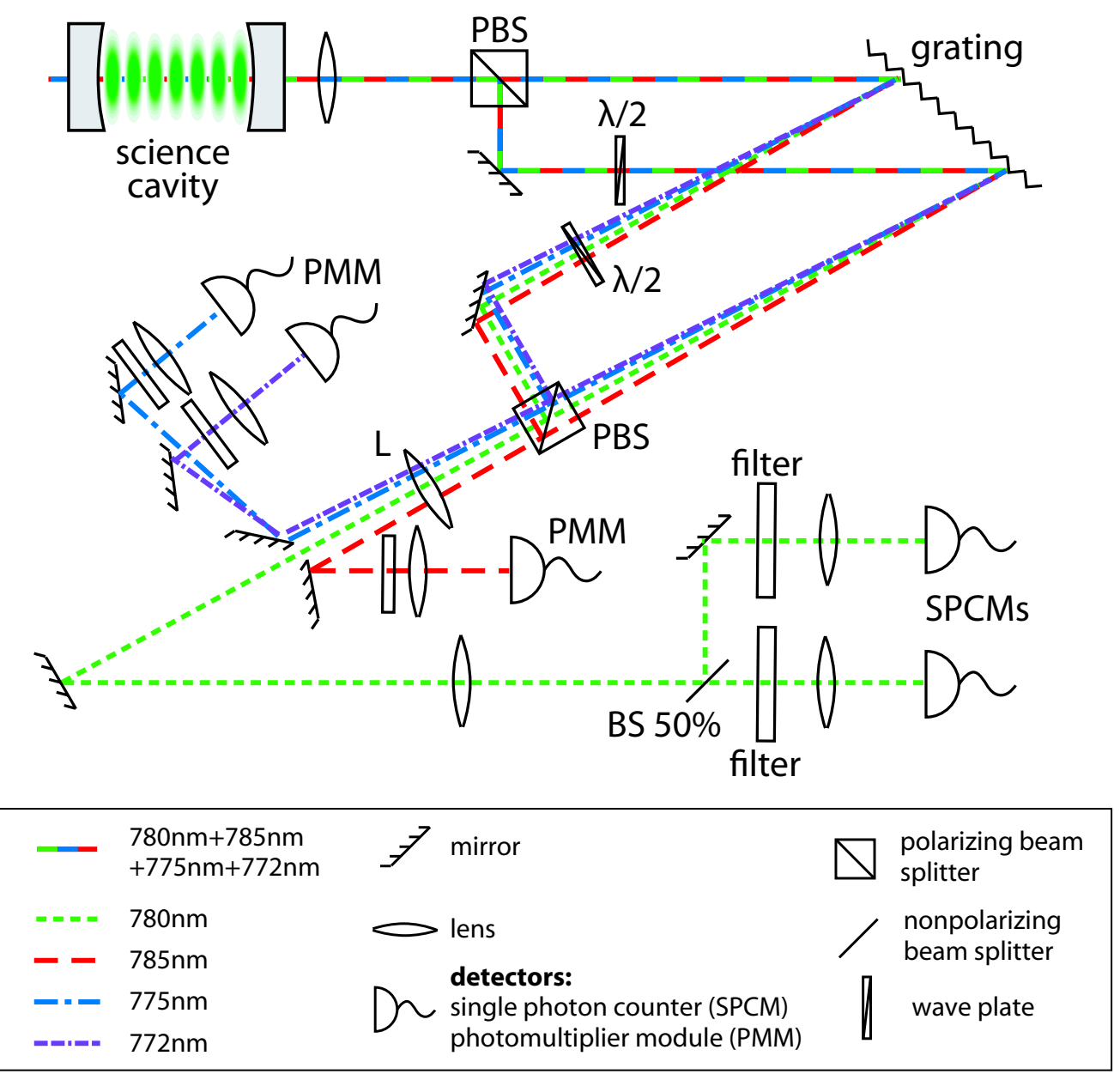

Figure 6.7: Detection behind the high-finesse cavity. The transmitted beams are separated by a holographic grating. To use the maximum efficiency of $90 \%$, the linear polarizations are split and adapted before the grating, and recombined afterwards with polarization optics. In the focal plane of a lens $(\mathrm{L}, f=150 \mathrm{~mm})$ the dipole lasers are reflected from edge mirrors to their corresponding photo-multiplier modules (PMM). To further reduce residual dipole light, the probe beam passes narrow bandpass interference filters before hitting the single-photon counters (SPCM).

physical parameters like laser frequencies and intensities are calibrated by gauge measurements. The results of the gauges, the parameter sets used and additional measurements during the sequences are stored in a relational database (PostgreSQL). Continuous traces, e.g. of the transmission of the probe and all dipole lasers are stored in separate files referenced in the database. They are later analyzed with additional evaluation programs and the results are again stored in the database.

The relational database allows for studies of parameter dependencies and systematics, which are of major importance because most experiments involve statistical analysis of 
a large number of events. The system runs stable for a few hours and data was taken continuously for about four months. 


\section{Chapter 7}

\section{Experimental realization of the blue trap}

In this chapter the realization of the blue-detuned intracavity dipole trap is discussed and the experimental results are presented (96). In Sec. 7.1 the concept of the bluedetuned light-field forming the trap is introduced. Next, a sample trace of a trapping event is presented in Sec. 7.2. For convenience the cavity is stabilized onto a very weak red-detuned stabilization laser, but a trap consisting of blue light only is also realized (Sec. 7.3). The essential feature of the blue trap is to preserve free-space properties for an atom stored in a region of strong coupling. The qualified strong coupling and the absence of the AC-Stark shift are directly observable in the normal-mode spectroscopy of the system (Sec. 7.4). The detection of the presence of an atom while it spontaneously scatters only about one photon is analyzed in more detail taking into account the Poissonian distribution of the detected photons (Sec. 7.5). The qualification of the coupling is discussed with respect to the spectrum of the cavity-like normal mode for larger detuning (Sec. 7.6). The Doppler-type dependence of cavity cooling on detuning is directly discernible by comparison of the transmission spectrum with the loss spectrum in Sec. 7.7. Finally, cavity cooling in the blue trap (Sec. 7.8) and trapping atoms in the parameter regime compatible with three-dimensional cooling (Sec. 7.9 ) are presented.

\subsection{Blue-detuned modes for guiding and trapping}

An atom is repelled by a blue-detuned light field. To achieve trapping of an atom at an intensity minimum, it must be completely surrounded by light. This generally renders a blue trap more complex than a red dipole trap. In a red dipole trap the atom is attracted to intensity maxima and it can therefore be realized by a simple beam focus or the antinode of a standing wave. To achieve guiding and trapping in a blue-detuned intracavity light field several modes have to be combined.

A high-finesse Fabry-Perot cavity only supports well defined sets of eigenmodes. In the paraxial approximation higher order cavity modes are given by a standing wave along the cavity axis and a transverse mode pattern generated by superpositions of, 
e.g., the Hermite-Gaussian basis (Sec. 9.1). The number of axial antinodes defines the longitudinal mode order. The fundamental mode is given by the $\mathrm{TEM}_{00}$ mode with a Gaussian profile. The frequency difference between consecutive longitudinal $\mathrm{TEM}_{00}$ modes is the free-spectral range (FSR). For the present cavity the FSR $/ 2 \pi=1.23 \mathrm{THz}$. In a cavity with ideal spherical mirrors the modes of one transverse order are degenerate. In the near-planar cavity used in the experiment, different transverse mode orders are separated by a few ten GHz. Therefore, the mode patterns in the transverse plane are superpositions of the Hermite-Gaussian modes spanning the given mode order. The manifold of order one is spanned by the $\mathrm{TEM}_{10}$ and $\mathrm{TEM}_{01}$ modes.

For trapping the intracavity dipole potential is created by a combination of standingwave cavity modes of different longitudinal and transverse mode order, see Fig. 7.1 and Fig. 3.3 for a three-dimensional impression. All modes are blue detuned with respect to the near-resonant cavity QED probe field: persistent axial confinement along the cavity axis is provided by a $\mathrm{TEM}_{00}$ mode (2) detuned by an odd number of free-spectral ranges (FSR). For an arriving atom the potential landscape looks like a one-dimensional array of mountains rising from the plane, which form long and narrow valleys in between.

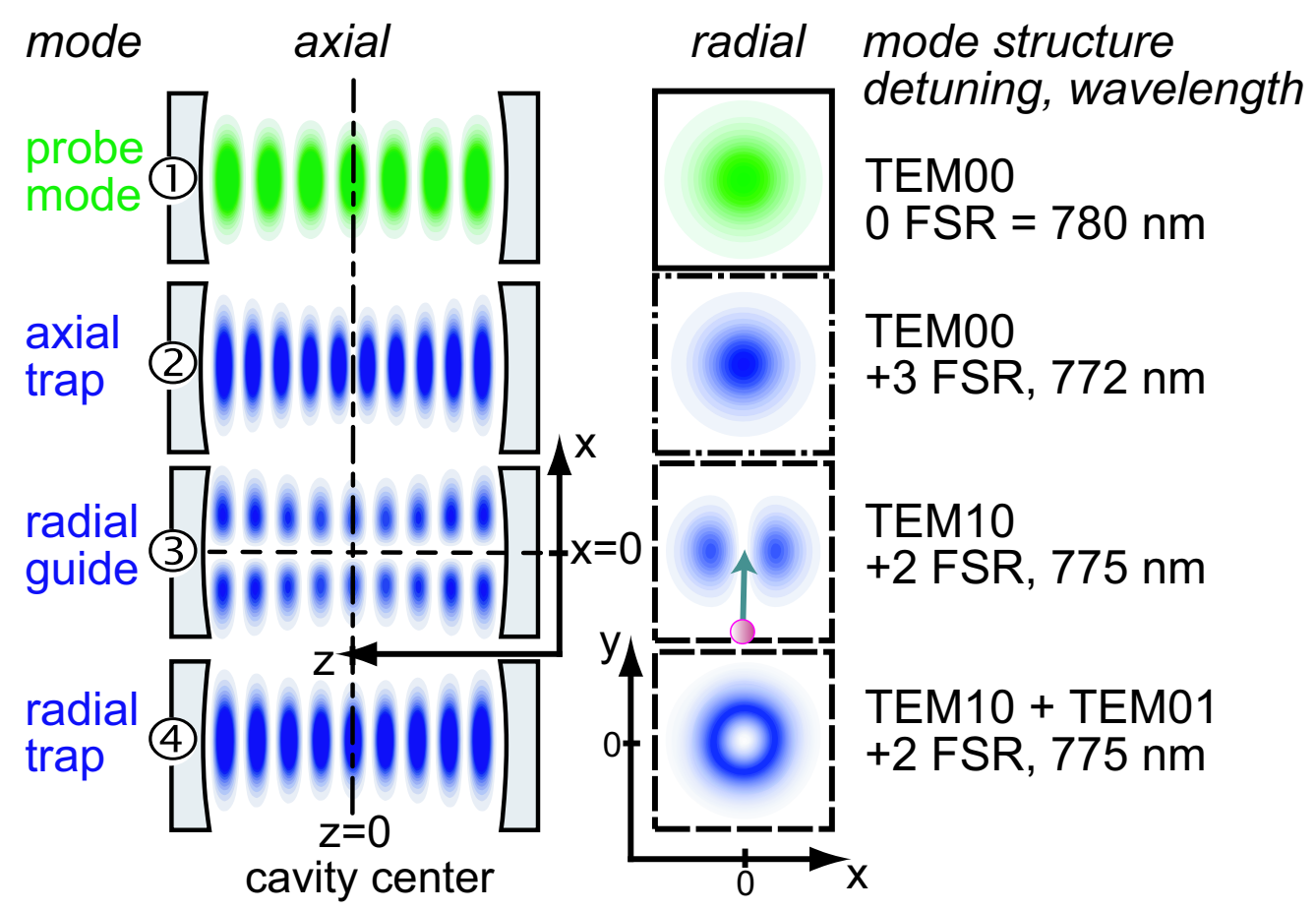

Figure 7.1: Blue intracavity dipole trap from the perspective of an entering atom. The slow atom is restricted to the field minima of the blue light fields that coincide with the antinodes of the near-resonant probe mode (1) at the cavity center. Persistent axial confinement is provided by a $\mathrm{TEM}_{00}$ mode (2), pancakes. Combined with the transverse nodal line of a $\mathrm{TEM}_{10}$ mode (3), funnels are formed to guide the atom to a strong-coupling region. Full three-dimensional confinement is achieved by adding a $\mathrm{TEM}_{01}$ mode to complete a transverse 'doughnut' mode (4). 
The very oblate antinodes - resembling a 'stack of pancakes' - confine the atom to the nodal planes. Halfway between the mirrors the nodal planes overlap with the antinodes of the probe mode (1). Transverse confinement is provided by a 'doughnut' mode (4) formed by a combination of $\mathrm{TEM}_{10}$ and $\mathrm{TEM}_{01}$ modes detuned by an even number of FSR. To load an atom into the trap, the transverse confinement is relaxed by using the $\mathrm{TEM}_{10}$ mode only. Slow atoms from an atomic fountain are injected from below along the $y$-direction. They are guided towards the cavity center at $x=0$ along the nodal line of this $\mathrm{TEM}_{10}$ mode (3). The combination of axial confinement and transverse guiding creates funnels that direct the atom to the antinodes of the probe mode. The trap is closed by adding the $\mathrm{TEM}_{01}$ mode to complete the transverse confinement. Note that the axial confinement need not be switched to close the trap. Since the axial and transverse characteristics of the trap are defined by independent modes at different laser frequencies, they can be controlled individually.

In a real cavity the degeneracy of the higher order modes is generally lifted by small imperfections. For the modes of order one, the commonly observed symmetry breaking with respect to two main axes can be explained by the main axes of the mirror curvature ellipsoids. The broken symmetry corresponds to the freedom in orientation of the two eigenmodes. Figure 7.2 shows the spectrum of the cavity modes of order one, which agree well with $\mathrm{TEM}_{10}$ (1) and $\mathrm{TEM}_{01}$ (2) modes oriented at about $-45^{\circ}$ and $+45^{\circ}$, respectively. These modes are split by about $6 \mathrm{MHz}$. The structure of the excited mode depends on the detuning as well as the mode matching of the pump mode. On resonance the respective eigenmode is predominantly excited. For off-resonant excitation both eigenmodes are pumped at the given frequency and the resultant mode is a superposition. The intensity and phase of the two contributions are given by the overlap with the pump. For the mode matching chosen in Fig. 7.2 the superposition mode that forms between the two eigenmodes is close to a $\mathrm{TEM}_{10}$ mode with the nodal lines oriented in the vertical direction. While between the resonances the two modes are excited with the opposite phase, there is no phase-shift between the excitation of the modes for large detunings $(|\Delta| \gg \kappa)$. Hence, in the far-detuned limit the excited intensity pattern is a $\mathrm{TEM}_{10}$ mode with the nodal line oriented in the horizontal. It is orthogonal to the former mode used for guiding the atom.

The doughnut mode pattern for complete transverse confinement consists of a bright ring with a dark center. Experimentally, it is formed by the sum of both eigenmodes each resonantly excited by independent laser beams. By choosing the relative intensities the shape and orientation of the doughnut can be fine tuned (Fig. 7.2 C.). The radial confinement is given by the interference of the two modes. the resulting potential is modulated in the azimuthal direction. At a given time, it consists of a ring with two minima and maxima in orthogonal directions. The orientation of this potential rotates with the difference frequency of the two laser beams. At the same time, the modulation depth varies. Full modulation is reached when both modes are excited with the same or opposite phase to form a $\mathrm{TEM}_{10}$ and $\mathrm{TEM}_{01}$ mode, respectively. At a phase of $\pm 45^{\circ}$ a normal doughnut is formed. Because the beat frequency of $6 \mathrm{MHz}$ is more than a magnitude larger than the typical radial oscillation frequency, the atom only sees the 

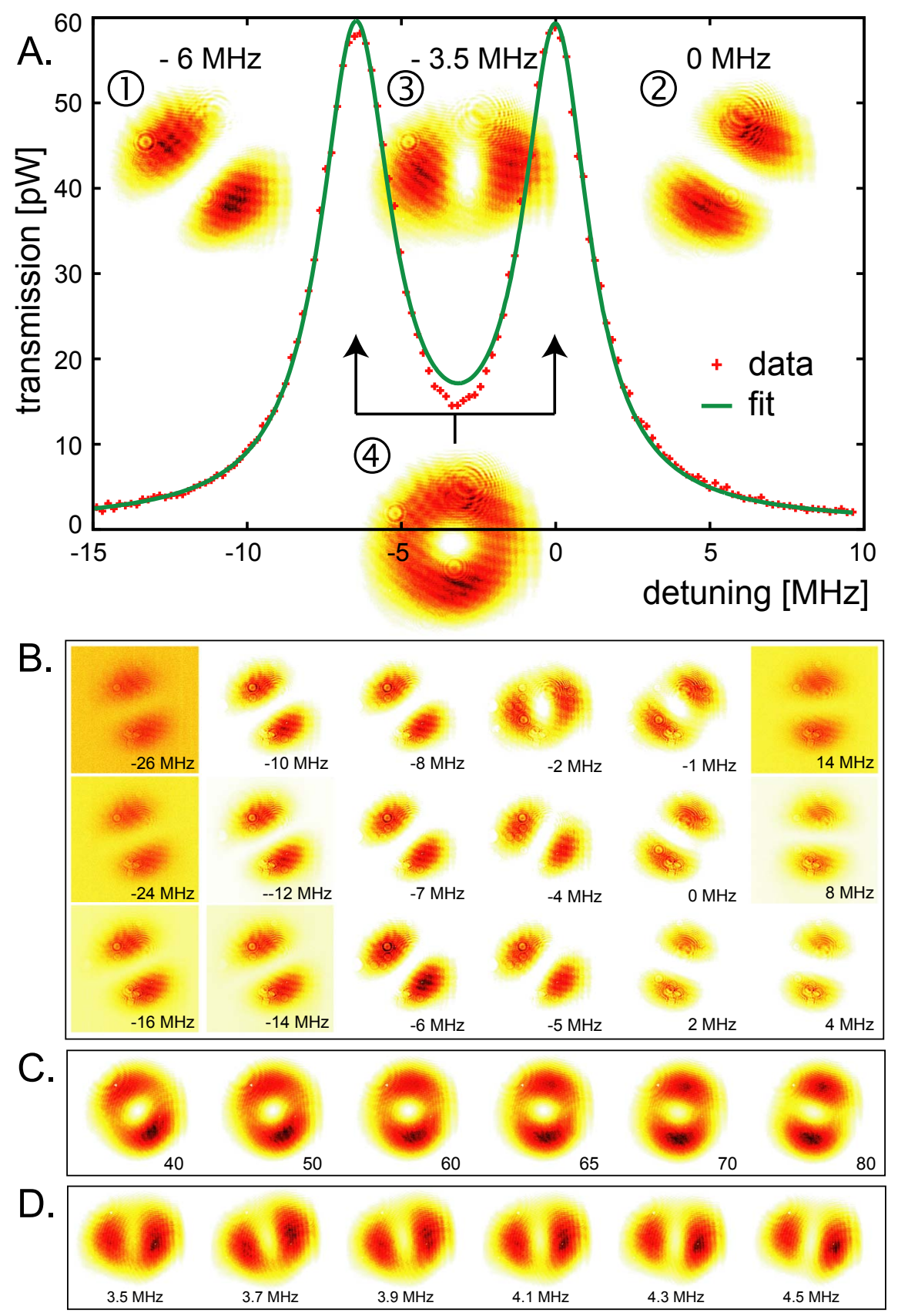

Figure 7.2: Spectrum of first-order modes. A. The spectrum of the first-order cavity modes consists of two orthogonal eigenmodes of the cavity which are close to the $\mathrm{TEM}_{10}+\mathrm{TEM}_{01}$ modes $(1)+(2)$ oriented at $\pm 45^{\circ}$ and split by $6 \mathrm{MHz}$. Exciting both modes at a frequency in between these resonances, with a relative phase given by the impinging $\mathrm{TEM}_{00}$ mode, allows to excite a superposition mode which resembles a $\mathrm{TEM}_{01}$ mode with the nodal line oriented vertically, appropriate to guide the atoms (3). By exciting both eigenmodes with two laser beams, the time-averaged excited light field corresponds to a bright doughnut (4) providing full transverse confinement. B. Scan of mode patterns excited at different detunings for a given mode matching. The superposition excited for large detuning $|\Delta| \gg \kappa$ is orthogonal to the guiding mode (3). C. The shape of the doughnut mode (4) can be fine-tuned by choosing the relative intensities of the two constituents [\%]. D. The orientation of the guiding mode depends on the detuning of the pump. 
time-averaged potential. By symmetry, the time-averaged potential is a complete regular doughnut as observed in the transmission in the experiment (Fig. 7.2). Note that since the potential is never anti-binding, stability is not an issue for this time-varying potential.

\subsection{Sample trace}

A sample trace of a trapping event is presented in Fig. 7.3. The cavity transmission of the near-resonant probe laser at $780.2 \mathrm{~nm}$ and of the blue-detuned dipole laser providing the transverse confinement are shown. The cavity is continuously stabilized on the weak, two free spectral-ranges (FSR) red-detuned, stabilization laser ECDL785 (not shown). The persistent axial confinement generated by a TEM ${ }_{00}$ mode at $772 \mathrm{~nm}$ (3 FSR detuned from the atom) (2) amounts to a maximum potential height of $U_{a}=h \times 346 \mathrm{MHz}$. The guiding field at $775 \mathrm{~nm}$ (2 FSR detuned from the atom) (3) produces a potential with height $U_{g}=h \times 2 \times 10.3 \mathrm{MHz}{ }^{\text {(i.). }}$. The probe laser is on resonance with the bare (ii.) cavity, $\Delta_{c}=0$. Thus, the presence of an atom detunes the cavity from resonance and causes a decrease in the transmission. Slow atoms are guided to regions of strong coupling and cause sharp transmission drops (5). The trigger is armed $t=205 \mathrm{~ms}$ after launch of the atoms from the atomic fountain to select late atoms arriving with velocities below $0.1 \mathrm{~m} \mathrm{~s}^{-1}$. Upon detection of a strongly coupled atom in the cavity center (A), the atom is trapped by converting the transverse guiding mode to a confining doughnut mode (4) with a maximum potential height of $h \times 30 \mathrm{MHz}$. Simultaneously, the probe laser intensity is reduced. When the atom leaves the mode, the cavity transmission increases to the bare cavity value for the reduced observation power (B). After each trapping event, the stabilization of all lasers and the cavity is checked (6)

\subsection{Sample trace for blue only trap}

In most experiments the cavity is stabilized to a +2 FSR red-detuned TEM $_{00}$ mode (ECDL785) which is independent of the blue-detuned trapping beams. This is convenient to study the blue trap, because the intensities of the blue modes can be freely adjusted without affecting the cavity stabilization. However, the weak red-detuned stabilization beam contributes a shallow trapping potential with an associated Stark shift in the order of a few MHz. Generally, the axial confinement provided by the ECDL775 is constant throughout the trapping events and therefore this laser is equally well suited for the cavity stabilization. To that end, the grating that splits the reflected light is tilted to direct the TiSa772 beam onto the avalanche photodiode. Since the trap beam is much stronger, it is attenuated and the gain is adjusted. Figure 7.4 shows a sample trace in a blue-only trap where the trap center is completely dark.

\footnotetext{
(i.) The maximum transverse trap height in Fig. 7.3 is given for the doughnut mode. Since it scales with the ratio of the maximum intensity to the integrated intensity, the trap height for the $\mathrm{TEM}_{01}$ guiding mode is a factor 2 larger.

(ii.) bare refers to the free-space subsystems,i.e. uncoupled and not trapped.
} 


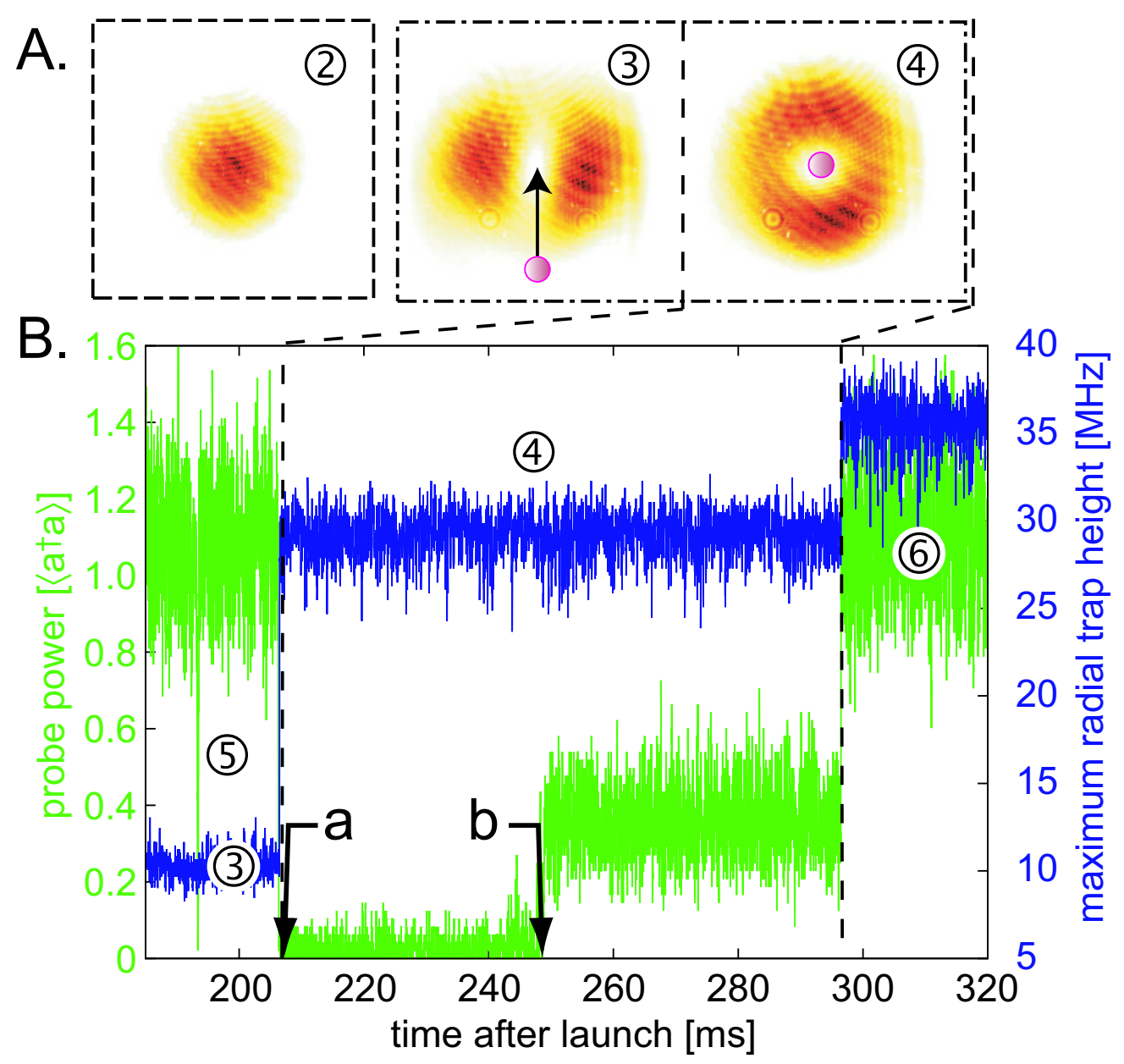

Figure 7.3: A blue-trapping event. A. Experimental intensity patterns of the different modes (cf.Fig. 7.1 \& 7.2). B. Sample trace: The transmitted probe power (lighter trace) in units of intracavity photon number $\left[\left\langle a^{+} a\right\rangle\right]$ (left scale) and the maximum transverse trap height (darker trace, right scale) are shown. The axial confinement of a $\mathrm{TEM}_{00}$ (+3 FSR) mode (2) combined with the nodal lines of a TEM 10 ( +2 FSR) (3) form funnels that guide single atoms to regions of strong coupling. Before the trigger is armed at $t=205 \mathrm{~ms}$, a single passing atom causes a steep dip in the cavity transmission (5). Upon detection of an atom (a) the probe intensity is decreased and the trap is closed by switching to a doughnut $\mathrm{TEM}_{10}+\mathrm{TEM}_{01}$ (4). When the atom leaves (b), the empty cavity transmission is observed. Finally, the stability of the system is checked (6) The noise on the transmission of the near-resonant probe is mainly shot noise. 


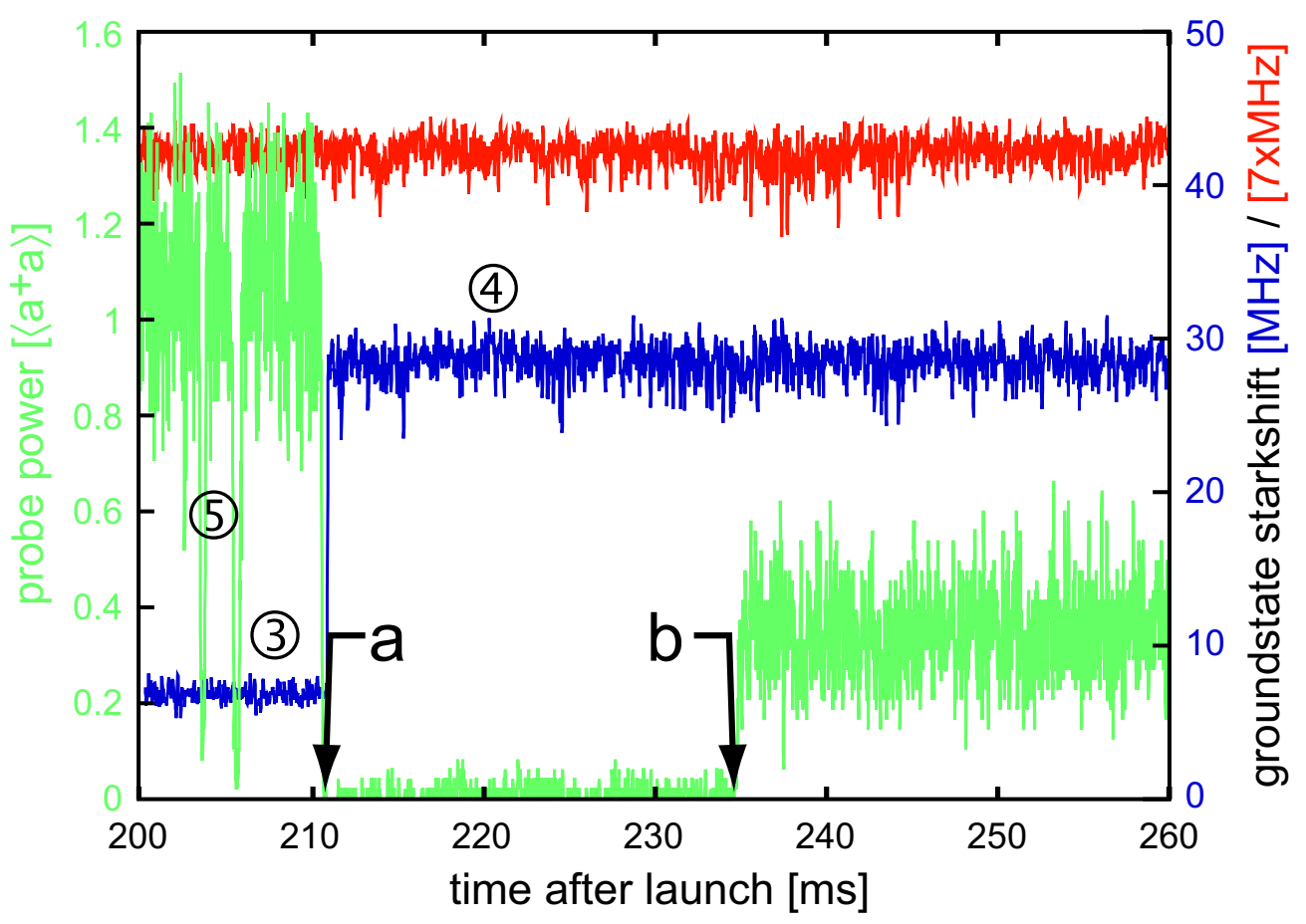

Figure 7.4: Sample trace for blue only trap. It is possible to omit the far red-detuned stabilization laser. Since the axial confinement need not be switched, the high-finesse cavity can be stabilized to the blue TiSa772laser. The traces of the trap modes for persistent axial TiSa772(red) and the switched radial ECDL775(blue) confinement correspond to Fig. 7.3 .

\subsection{Normal-mode splitting}

To characterize the coupling and the Stark shift in the blue trap, Fig. 7.5 shows the normal-mode splitting of the qualified well-coupled atom-cavity system. The transmission spectrum is measured by alternating $0.5 \mathrm{~ms}$ long cooling and $0.1 \mathrm{~ms}$ short probing intervals. See Fig. 7.9 for a sample trace of the measurement sequence. The probe laser detuning in the probe intervals is scanned with respect to the bare cavity frequency, which is $\Delta_{a c} / 2 \pi=35(1) \mathrm{MHz}$ blue detuned from the atomic frequency, where the uncertainty comes from the Zeeman shift in the residual magnetic field. During the cooling intervals the probe laser is on resonance with the bare cavity $\left(\Delta_{\mathrm{c}}=0\right)$, which allows for cavity cooling in the axial direction as well as independent qualification of the atomcavity coupling. Details on the qualification can be found in section 7.6. A probe interval qualifies for strong atom-cavity coupling, when the cavity transmission in the neighboring cooling intervals is below $10 \%$ of the bare cavity transmission. The expectation value of the photon number in the cavity mode is calculated from the measured photondetection rate and the known detection efficiency, including propagation losses from the cavity to the detectors. Experimental results are displayed in Fig. 7.5. Analytical results for an atom with fixed coupling at low excitation (solid curve) fit the data (points) well. 
Comparison between theory and experiment gives an atom-cavity coupling constant of $83(12) \%$ of the maximum possible value at a cavity antinode, $g_{0} / 2 \pi=16 \mathrm{MHz}$, much larger than the atomic and cavity decay rates. This proves that a strongly-coupled atom-cavity system has been prepared. The empty cavity Lorentzian centered at $0 \mathrm{MHz}$ is shown for reference. In this case the probe intervals are qualified in the opposite fashion to be above $90 \%$ of the empty cavity transmission.

The normal-mode spectrum measured in the red trap for the same free-space detunings of the subsystems is shifted close to the degenerate case (see also (94) and Sec. 8.2). In a red trap the atomic resonance is shifted by approximately twice the ground-state trap depth, effectively bringing the atomic transition frequency close to resonance with the cavity. The close to symmetric normal modes are dynamically broadened and well described by the result of numerical stimulations. In contrast, the normal-mode spectrum of an atom stored in the blue-detuned trap does not show any shift of the atomic frequency, as expected for an atom trapped at the node of the blue field. Since the large atom-cavity detuning is preserved, the character of the normal modes emerging from the bare states remains largely atom-like and cavity-like. The strong asymmetry of the peak heights arises from the fact that the system is excited via the cavity and observed in transmission. Therefore, the atom-like resonance is much weaker than the cavity-like resonance. The plots on shaded background are a blow-up of the spectrum $(\sim 130 \times)$ to present the atom-like peak located at approximately $\Delta_{\mathrm{c}} / 2 \pi=-40 \mathrm{MHz}$. This peak is broadened by the spatial distribution of the atoms in the mode. An analytical fit for a fixed atom to the data including a Stark shift results in a coupling $g=85(13) \% \times g_{0}$ and a Stark shift of $\Delta_{\mathrm{S}} / 2 \pi=0.25(1.42) \times g_{0}$. A conservative upper bound for the Stark shift of $\Delta_{\mathrm{S}} / 2 \pi<4 \mathrm{MHz}$ can be extracted by fitting the slopes of the atom-like normal mode only. The Stark shift is hence much smaller than the axial and transverse trap heights, $U_{a}=h \times 265(6) \mathrm{MHz}$ and $U_{r}=h \times 30(1) \mathrm{MHz}$, respectively. The Stark shift due to the red-detuned stabilization laser at $785.2 \mathrm{~nm}$ is $\Delta_{\mathrm{stab}} / 2 \pi=2.2(1) \mathrm{MHz}$. Thus, the shift of the atomic transition frequency due to the blue trap is smaller than the atomic linewidth.

\subsection{Single atom detection}

One of the fascinating capabilities readily accessible in the strong-coupling regime is the possibility to detect a single atom in a cavity with a high bandwidth (100). The motion of a single bound atom in a cavity can be observed (101). Alternatively, the state of a localized atom can be detected (130). High-finesse cavities are well suited for single atom detectors in integrated systems (131; 132, 133; 134). The analysis of the statistical properties of an atom laser coupled out of a Bose-Einstein condensate is a first experimental application (135). In the low excitation limit the signal generally scales with the excitation but at the same time heating due to spontaneous emission increases. Hence, a benchmark for detection is the achievable bandwidth at a given atomic excitation. Of particular interest is resonant dispersive detection, where the probe is resonant with the empty cavity but detuned from the atom. Two effects contribute 


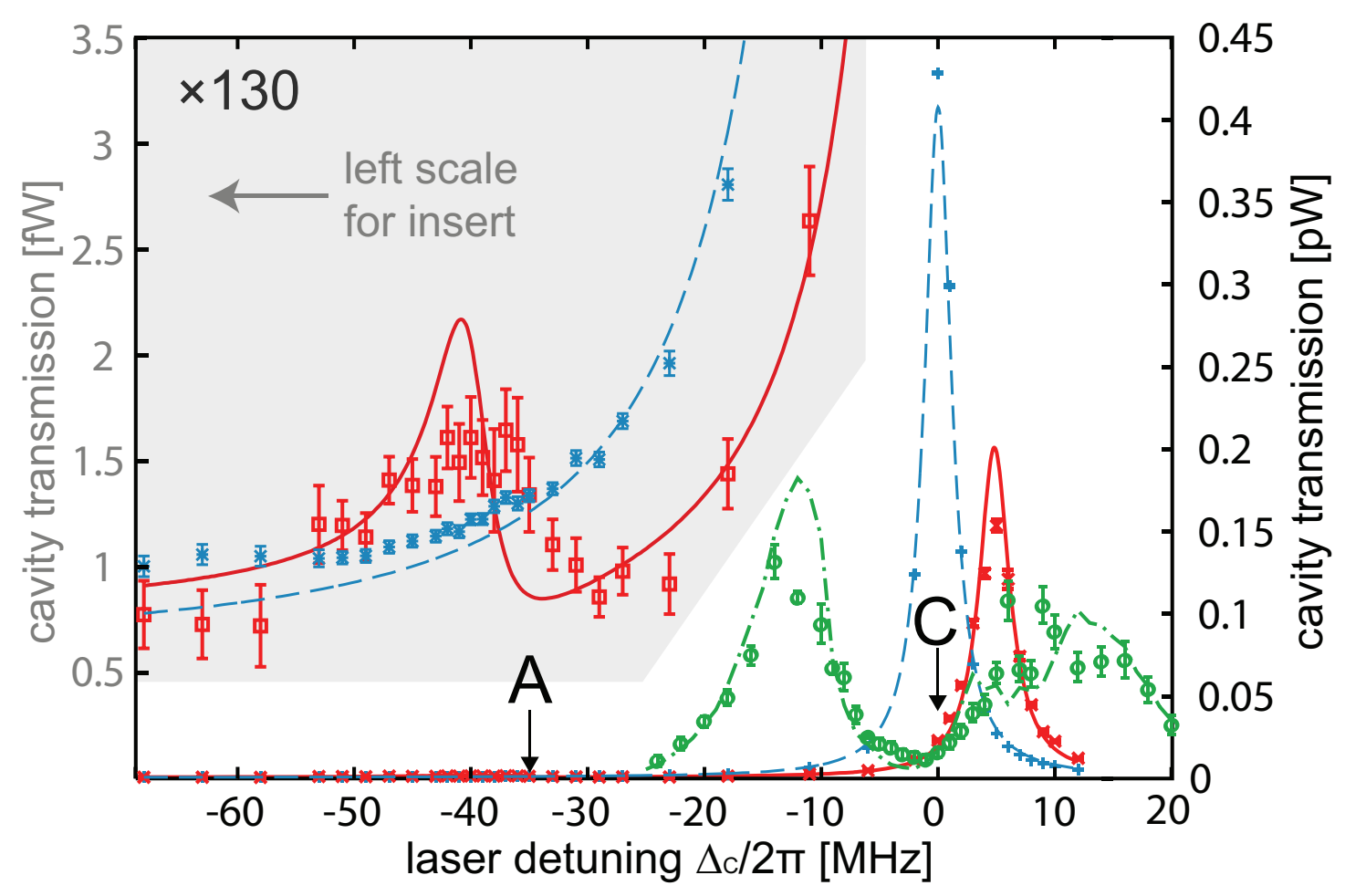

Figure 7.5: Normal-mode splitting for $\Delta_{a c} / 2 \pi=-35 \mathrm{MHz}$. The average transmission of a well-coupled system shows a well resolved normal-mode splitting (red squares and crosses). The bare atom (A) is detuned from the bare cavity resonance (C) by $\Delta_{a c} / 2 \pi=-35 \mathrm{MHz}$. A transmission of $1 \mathrm{pW}$ corresponds to 1.2 intracavity photons. Intervals contribute to the spectrum if the transmission in the neighboring cooling intervals is $\left\langle 10 \%\right.$ of the bare cavity value $\left\langle n_{0}\right\rangle$. An analytical fit (solid line) for a fixed coupling $g$ at low excitation results in $g=0.83(12) \times g_{0}$ and a residual Stark shift of $\Delta_{\mathrm{S}} / 2 \pi=0.7(1.3) \mathrm{MHz}$. The empty cavity transmission Lorentzian at $0 \mathrm{MHz}(\mathrm{C})$ is shown for reference.

The insert shows the same curves magnified by $\sim 130 \times$ to make the atom-like normal mode visible. A fit to the slopes of this peak leads to a conservative upper bound of the Stark shift in the blue trap of $\Delta_{\mathrm{S}} / 2 \pi<4 \mathrm{MHz}$.

The normal-mode spectrum measured in the red trap for the same bare detunings is shown for comparison (green circles). Due to the Stark shift in the trap, the atom is effectively shifted close to resonance with the cavity. The near-symmetric normal modes are broadened by the dynamics of the atom and are well reproduced by the numerical Monte Carlo simulations (green dashed-dotted line). 
in favor of detection in this regime as discussed next, and further analyzed taking into account the Poissonian distribution of the photon detection.

\subsubsection{Resonant dispersive detection}

The preserved large atom-cavity detuning $\left(\Delta_{a c}>2 g\right)$ facilitates dispersive measurements (98; 99), while at the same time the blue trap provides strong confinement. This is exemplified by the detection of an atom in the cavity via the induced shift of the cavity-like normal mode. To estimate the average number of spontaneously scattered photons during a certain observation time interval, we consider probing the system on resonance with the bare cavity $\left(\Delta_{\mathrm{c}}, \Delta_{\mathrm{a}}\right) / 2 \pi=(0,-35) \mathrm{MHz}$. In the presence of a strongly coupled atom, the cavity transmission of the probe is reduced by a factor of 20.3(5). The transmission is a direct measure of the excitation of the mode corresponding to $\left\langle a^{+} a\right\rangle$ photons. In the limit of weak excitation, the excitation probability of the atom is proportional to the photon number in the mode times the atomic Lorentzian: $\left\langle\sigma^{+} \sigma^{-}\right\rangle=\left\langle a^{+} a\right\rangle g^{2} /\left(\Delta_{a c}^{2}+\gamma^{2}\right)$, cf. eqn. 2.47. The atomic excitation $\left\langle\sigma^{+} \sigma^{-}\right\rangle$is therefore given by the cavity excitation $\left\langle a^{+} a\right\rangle=0.022$ (cf. Fig. 7.5 C) times a constant which depends on the effective coupling, $g$, the atom-cavity detuning, $\Delta_{a c}$, and the atomic linewidth, $\gamma$. The effective coupling was obtained from the experimental data in Fig. 7.5 , and $\gamma$ and $\Delta_{a c}$ are well known. The average atomic excitation of $\left\langle\sigma^{+} \sigma^{-}\right\rangle=3.1 \times 10^{-3}$ leads to a scattering rate into free space given by $2 \gamma\left\langle\sigma^{+} \sigma^{-}\right\rangle \approx 117 \mathrm{kHz}$. Thus, during a time interval of $10 \mu \mathrm{s}$ the atom scatters $1.2(3)$ photons. A detailed analysis in the next Subsec. 7.5.2, which takes into account the Poissonian statistics of the detected photons, results in a $95 \%$ correct decision concerning the presence of the atom in this $10 \mu$ s long time interval. This includes an overall experimental detection efficiency of $5 \%$ for photons lost from the cavity mode. The required observation time interval scales inversely with the photon-detection efficiency which can be improved considerably. Detecting the (lack of) photons emitted from the cavity does not rely on the excitation of the atom and is in that sense complementary to cavity cooling.

The efficient detection of a single, well-coupled atom on resonance with the bare cavity at large atom-cavity detuning, $\left(\Delta_{c}, \Delta_{a c}\right) / 2 \pi=(0,-35) \mathrm{MHz}$, relies on two effects. First, the presence of the atom reduces the probe transmission by more than an order of magnitude $\left(1+\left|g^{2}\right| /\left(\kappa \Delta_{a c}\right)\right)^{-1} \simeq 0.05$ leading to a high signal-to-noise ratio as well as reducing the excitation of the system. Second, the fact that the bare atomic state is far detuned results in an additional reduction of the excitation of the atom by a factor of $\left(|g| / \Delta_{a c}\right)^{2} \simeq 0.14$ with respect to the cavity excitation. Since this effect increases with the detuning of the bare atom $\Delta_{a c}$, while the reduction in the cavity excitation due to the coupled atom decreases, there is an optimum value for $\Delta_{a c}$. The analysis in the next section will show that the detection efficiency is about the same when going to a larger detuning of $\Delta_{\mathrm{a}}=50 \mathrm{MHz}$. In fact, using eqns. 2.47 and 2.46 it can be shown that for constant atomic excitation the detection efficiency is constant to first approximation once $\Delta_{a c}$ is larger than $g$. Note that the detection bandwidth can directly be increased with larger probe power resulting in higher count rates, but unfortunately, this will increase the transverse heating and decrease the lifetime of the atom in the trap accordingly. 


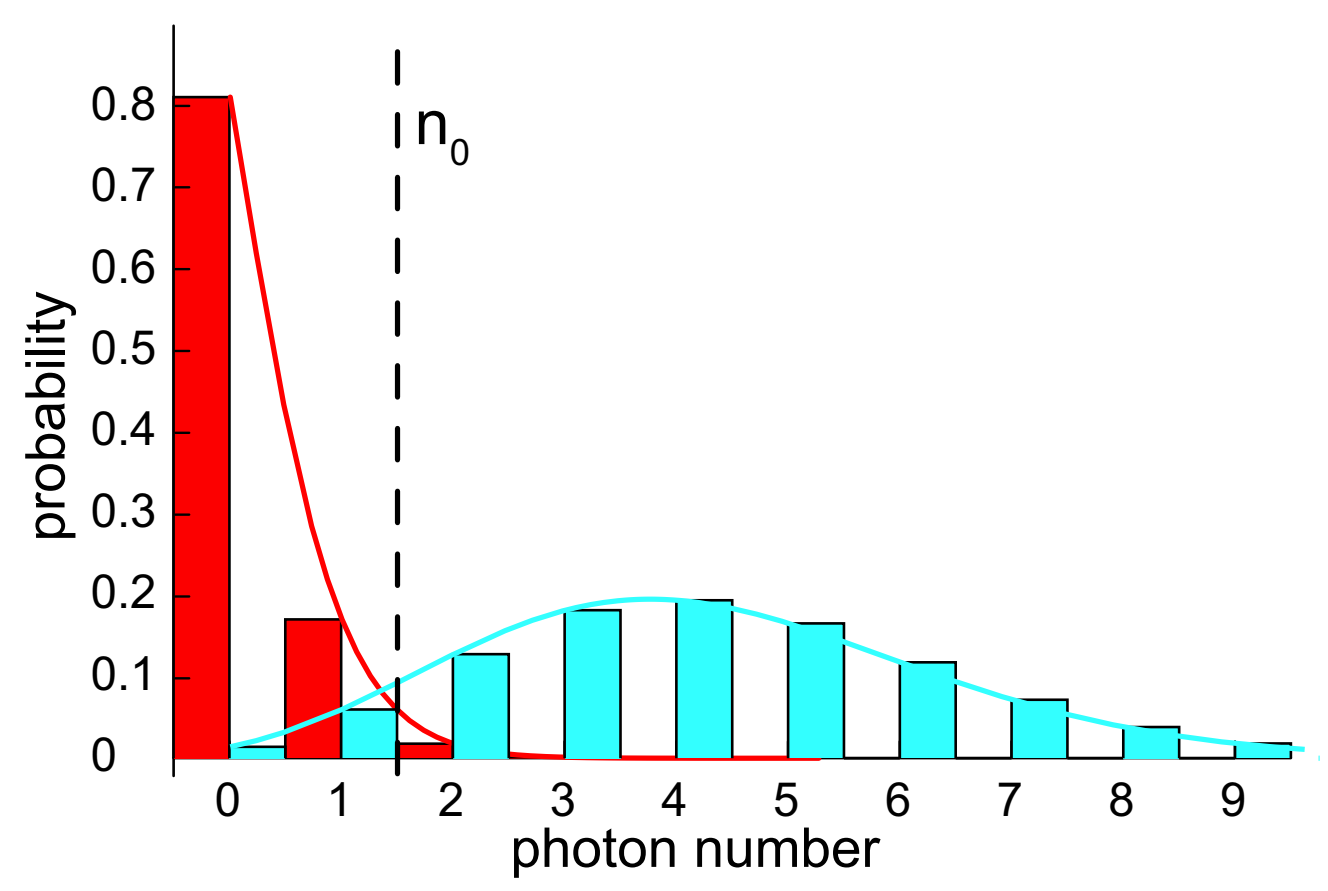

Figure 7.6: Poissonian distributions for the mean number of detected photons in an interval of length $\tau=10 \mu \mathrm{s}$ for the empty cavity (blue) and a well-coupled atom (red). A threshold photon number $n_{0}$ is chosen such that for $n \leq n_{0}\left(n>n_{0}\right)$ a strongly coupled atom is assumed to be present (not present).

\subsubsection{Poisson analysis}

A more detailed analysis of the time necessary to detect the presence of an atom takes into account the Poissonian statistics of the emitted photons. For the given parameters in the experiment the cavity mode is to very good approximation in a coherent state. Therefore, the mean photon detection rates for 'no atom' $r_{\text {nat }}=426.8(4) \mathrm{kHz}$ and 'one well-coupled atom present' $r_{\text {at }}=21.0(5) \mathrm{kHz}$ are extracted from fitting Poissonian distributions to the measured qualified data. The photon distributions for the different detunings and the fitted mean values are summarized in Fig. 7.7 for the normal-mode spectra at $\Delta_{\mathrm{a}} / 2 \pi=-35 \mathrm{MHz}$ and at $\Delta_{\mathrm{a}} / 2 \pi=-50 \mathrm{MHz}$.

For a given detection time $\tau$ the mean number $\mu$ of detected photons is $\mu=\tau r$. The probability to detect $n$ photons is given by the Poisson distribution $P(n, \mu)$ with a mean photon number of $\mu$. Figure 7.6 shows the Poisson distribution for the experimentally detected mean numbers of photons in the cases of no atom $\mu_{\text {nat }}=4.268(4)$ and one well-coupled atom present $\mu_{\text {at }}=0.210(5)$ for $\tau=10 \mu \mathrm{s}$.

To decide on the question whether an atom is present, we set a limit of $n_{0}$ photons, such that for $n \leq n_{0}\left(n>n_{0}\right)$ we conclude, "yes" ("no"), an atom is (not) present. The probabilities for a correct $P_{\text {atc }}$ and incorrect $P_{\text {atnc }}$ decision about the presence of an atom and the corresponding quantities for the absence of an atom $P_{\text {natc }}$ and $P_{\text {natnc }}$, respectively, are given by: 


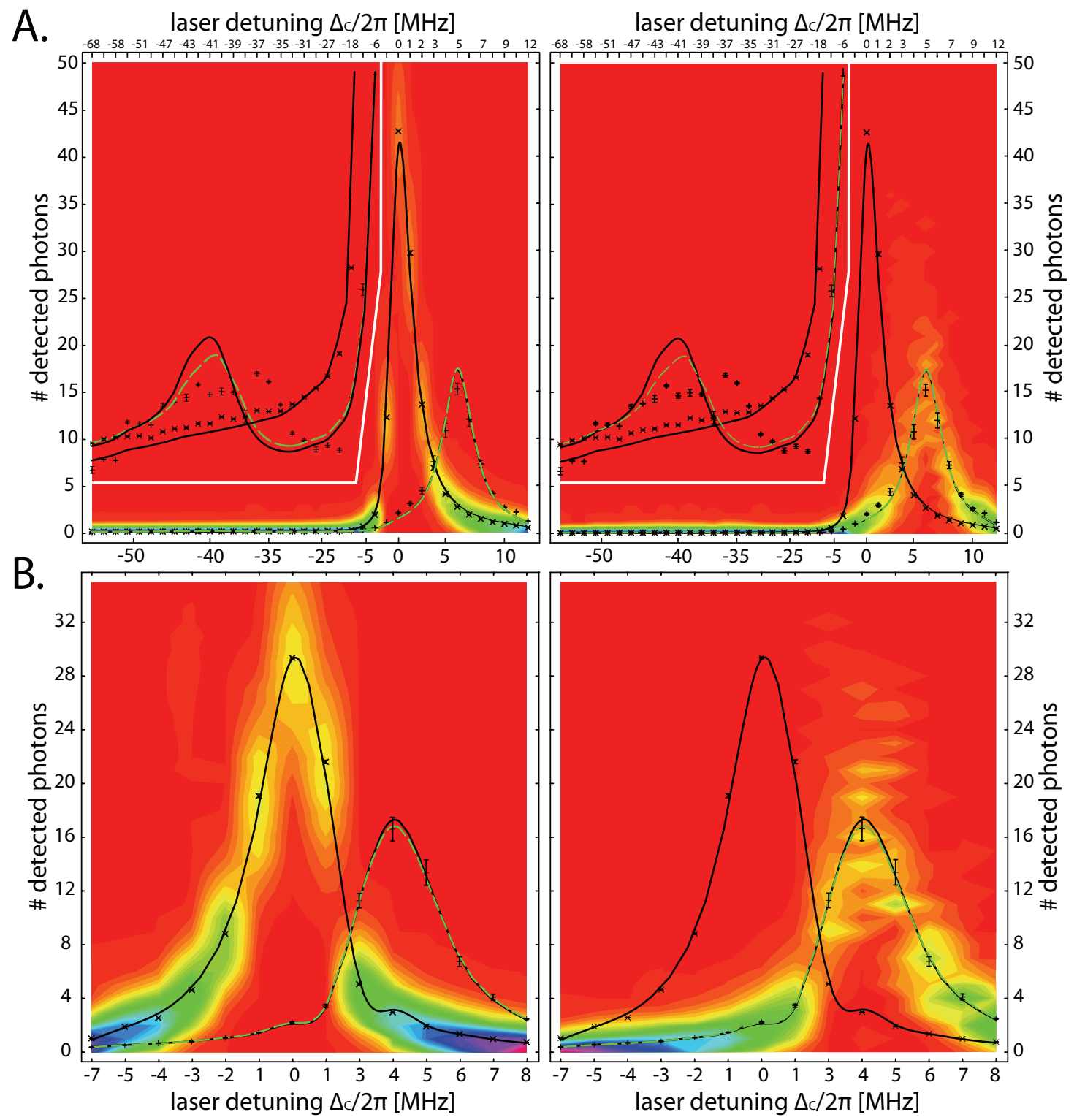

Figure 7.7: Normal-mode splitting: Poisson statistics. A. for $\Delta_{a c} / 2 \pi=-35 \mathrm{MHz}$ and B. for $\Delta_{a c} / 2 \pi=-50 \mathrm{MHz}$. The distribution of photons observed in the $0.1 \mathrm{~ms}$ detection intervals is plotted in color code (left: uncoupled system, right: coupled system). The horizontal axis is distorted because only the measured detunings are included. The points are the mean values of a fitted poissonian distribution with $95 \%$ confidence intervals. As expected for these parameters, the photon field is in good approximation in a coherent state. The residual systematic experimental broadening is small. The qualified transmission shows a well resolved normal-mode spectrum. The empty cavity is at $0 \mathrm{MHz}$, the bare atom at $\Delta_{\mathrm{a}} / 2 \pi=-35 \mathrm{MHz}$ and $-50 \mathrm{MHz}$, respectively. The analytical fits are included by quadratic splines (with Stark shift dashed green, without solid black). They result in a coupling of $g=83(12) \% \times g_{0}$ and a residual stark shift of $\Delta_{\mathrm{S}} / 2 \pi=0.7 \pm 1.3 \mathrm{MHz}$ for the $\Delta_{\mathrm{a}} / 2 \pi=-35 \mathrm{MHz}$ spectrum. The atom-like normal-mode is magnified in the inset (white box). 


$$
\begin{array}{ll}
P_{\text {atc }}=\sum_{n=0}^{n_{0}} P\left(n, \mu_{\mathrm{at}}\right), & P_{\mathrm{atnc}}=\sum_{n=n_{0}+1}^{\infty} P\left(n, \mu_{\mathrm{at}}\right) \\
P_{\text {natnc }}=\sum_{n=0}^{n_{0}} P\left(n, \mu_{\mathrm{nat}}\right), & P_{\text {natc }}=\sum_{n=n_{0}+1}^{\infty} P\left(n, \mu_{\text {nat }}\right) .
\end{array}
$$

For a statistical analysis of the probability of a correct decision, we have to assume an a priori probability $P_{\text {apri }}$ for an atom being present. The probability to correctly determine the presence of an atom is then given by:

$$
P_{\mathrm{c}}=P_{\text {apri }} P_{\text {atc }}+\left(1-P_{\text {apri }}\right) P_{\text {natc }} .
$$

In the following we assume an a priori probability $P_{\text {apri }}=0.5$ for the presence of the atom in the cavity, which corresponds to maximum possible ignorance. This is a rather conservative assumption given that the atom was recently conditioned to be there. A refined analysis would include the trapping efficiency and life time with multiple conditioned probabilities, which would get rather specific and involved. Figure 7.8 shows the dependence of the probability for a correct determination on the length of the detection interval $\tau$. For small $\tau$ the optimum reference level is $n_{0}=0$. With increasing detection interval length $\tau$, at some point even detecting one photon will more likely indicate the presence of an atom. For each $\tau$ the threshold photon number $n_{0}$ is chosen to optimize $P_{\mathrm{c}}$. In Fig. 7.8 two sets of curves are shown for the different atom-cavity detunings $\Delta_{a c} / 2 \pi=-35 \mathrm{MHz}$ upper and $\Delta_{a c} / 2 \pi=-50 \mathrm{MHz}$ lower set, respectively. The optimal probability for correct determination $P_{\text {c,opt }}$ are shown for a continuous $n_{0} \in \mathbb{R}_{>0}$ and for the discrete optimal $n_{0} \in \mathbb{N}$ (non-continuously differentiable curve). The optimal choice of $n_{0}$ is plotted in the inset for $\Delta_{a c} / 2 \pi=-35 \mathrm{MHz}$. The lines represent the linear increase in the mean number of spontaneously scattered photons during the time $\tau$. The analysis shows that a $10 \mu \mathrm{s}$ long detection interval, as in the last subsection, is a reasonable choice and allows to detect the presence of the atom while it spontaneously scatters only about one photon.

\subsection{Qualification}

To achieve good localization of the atom even in the presence of unavoidable heating during the probing of the system at different detunings, the probe intervals are surrounded by longer intervals of cavity cooling. Cavity cooling restores good localization in the axial direction. For cooling the probe is on resonance with the bare cavity (CRI), such that the transmission drops with increasing coupling. The coupling in the probe intervals can thus be independently qualified by a transmission below a threshold fraction of the empty cavity in the neighboring cooling intervals, see Fig. 7.9. This type of qualification strongly reduces the contribution of weakly coupled atoms (87). Due to the timescales it can only qualify for the radial motion it is therefore advantageous that 


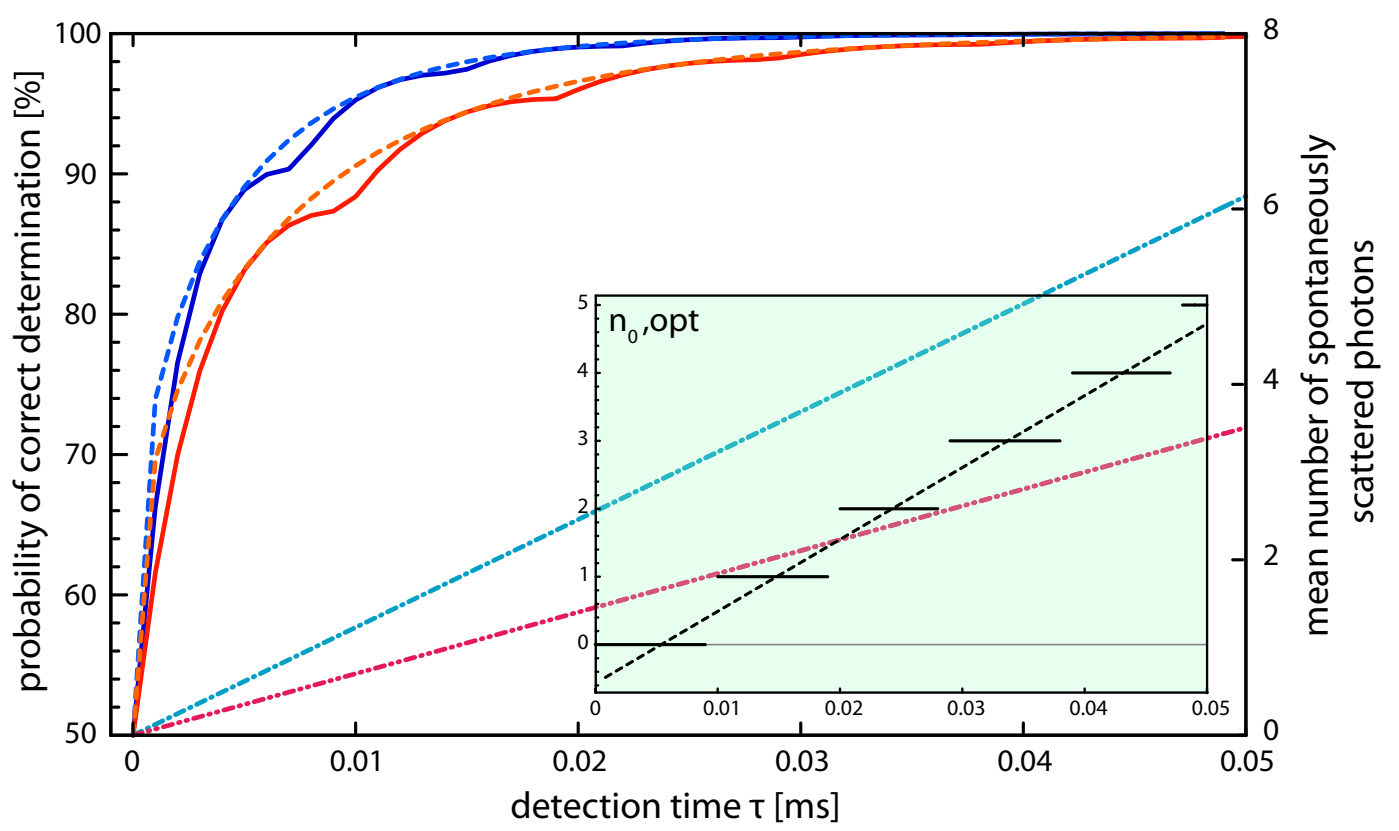

Figure 7.8: Single atom detection. Optimum detection probability $P_{\mathrm{c}, \mathrm{opt}}$ for $\Delta_{a c} / 2 \pi=$ $-35 \mathrm{MHz}$ in blue and $\Delta_{a c} / 2 \pi=-50 \mathrm{MHz}$ in red (left scale) provided an optimal threshold $n_{0}$ is chosen (Fig. 7.6). Note that the curves start at the a priori probability of $50 \%$ for $\tau=0$. The dashed-dotted lines show the corresponding mean number of spontaneously scattered photons (right scale). The inset shows the optimum choice for $n_{0}$ (for $\Delta_{a c} / 2 \pi=-35 \mathrm{MHz}$ ). A probability exceeding $95 \%$ is for example achieved for a detection time $\tau=10 \mu \mathrm{s}$ for $\Delta_{a c} / 2 \pi=-35 \mathrm{MHz}$ and $\tau=17 \mu$ s for $\Delta_{a c} / 2 \pi=-50 \mathrm{MHz}$ while the atom spontaneously scatters 1.23 and 1.19 photons, respectively. The solid (dashed) lines correspond to a discrete (continuous) choice of $n_{0}$.

the blue trap allows for strong axial confinement. The empty cavity can, conversely, be qualified by a transmission greater than a threshold fraction close to unity.

As an example, Figure 7.10 shows the effect of increasing qualification on the cavitylike normal-mode peak for a larger atom-cavity detuning of $\Delta_{a c} / 2 \pi=-50 \mathrm{MHz}$. The transmission of the empty cavity $(\mathrm{C})$ is approximated by the empty cavity Lorentzian. The other curves represent the data for increasing qualification. A probe interval is included in the calculation of the averaged transmission if the transmission in both neighboring cooling intervals $n_{\mathrm{c}}$ is below a fraction $\xi$ of the empty cavity transmission $n_{\mathrm{c}}<\eta \times\left\langle n_{0}\right\rangle$. The distribution of the transmission in the cooling intervals shows a bimodal structure, such that there are only very few intervals with qualification $0.1 \times$ $\left\langle n_{0}\right\rangle<n_{\mathrm{c}}<0.9 \times\left\langle n_{0}\right\rangle$. With increasing coupling of the atom the transmission on the bare cavity at $0 \mathrm{MHz}$ is reduced while the transmission on the normal mode at $4 \mathrm{MHz}$ increases. The analytical results for an atom with fixed coupling fit the data well. The result for the coupling is $g=0.87(1) \times g_{0}$ and $g=0.85(12) \times g_{0}$ for a fit without and with a fitted Stark shift of $\Delta_{\mathrm{S}} / 2 \pi=0.3(1.4) \mathrm{MHz}$. 

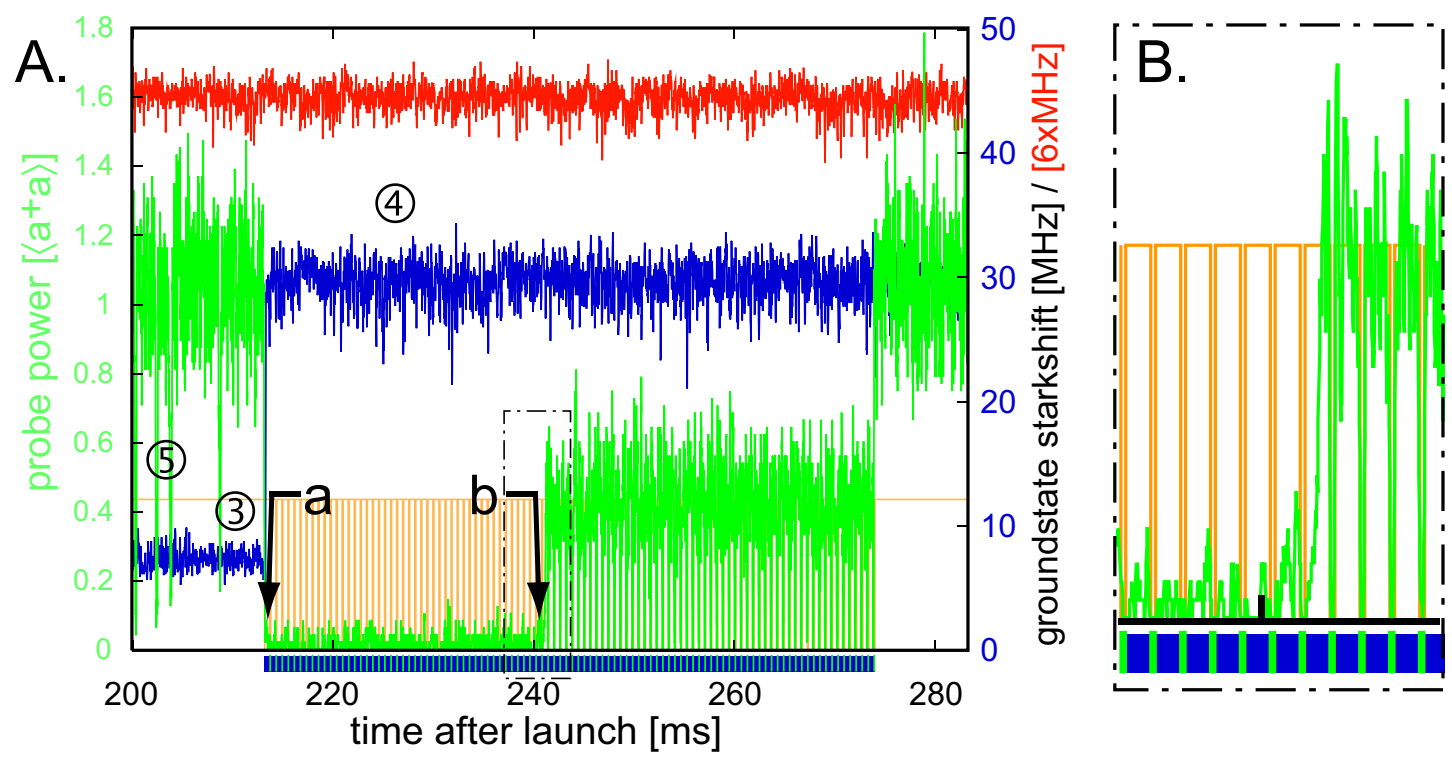

Figure 7.9: Sample trace for multiple switching. A. To compensate for strong heating for certain detunings and to restore good coupling the $0.1 \mathrm{~ms}$ short probe intervals are surrounded by $0.5 \mathrm{~ms}$ long intervals of cavity cooling. During the cooling intervals the probe in on resonance with the bare cavity (CRI). The coupling in the probe intervals can then be independently qualified for good coupling by the transmission during the neighboring cooling intervals to be below a threshold fraction $\xi$ of the empty cavity. In this particular case, the detuning in the probe intervals is largely detuned as can be seen by the dips, when the atom has left the mode after (b). The traces of the trap modes TiSa772(red) and ECDL775(blue) correspond to Fig. 7.4 and Fig. 7.3. B. Zoom into the transmission trace where the atom leaves the trap. The transmission during the cooling intervals returns to the empty cavity value which is the reference for the qualification. It corresponds to the upper level of the multiple step function which indicates the alternating sequence of intervals also shown in the bar below the time axis.

\subsection{Velocity-dependent forces}

Cavity cooling can be understood as Doppler-like cooling on the normal modes of the coupled system (see Sec. 4.1). The sign of the induced velocity-dependent force can be deduced from the energy balance. A probe laser red detuned with respect to the resonance of the system will lead to cooling and blue-detuned to heating. The major advantage of cavity cooling is that the excitation and dissipation necessary to remove energy and entropy from this system is preferentially realized via the cavity contribution of the dressed state. The excitation of the atom and spontaneous emission are unnecessary side effects, which can in principle be reduced by scaling both the coupling and the detuning. Appropriate detunings are thus given when the probe is red detuned with respect to the cavity-like normal mode.

The analogy of cavity cooling to Doppler cooling on the resonances of the coupled system is directly observed in Fig. 7.11. It shows the qualified average transmission and 


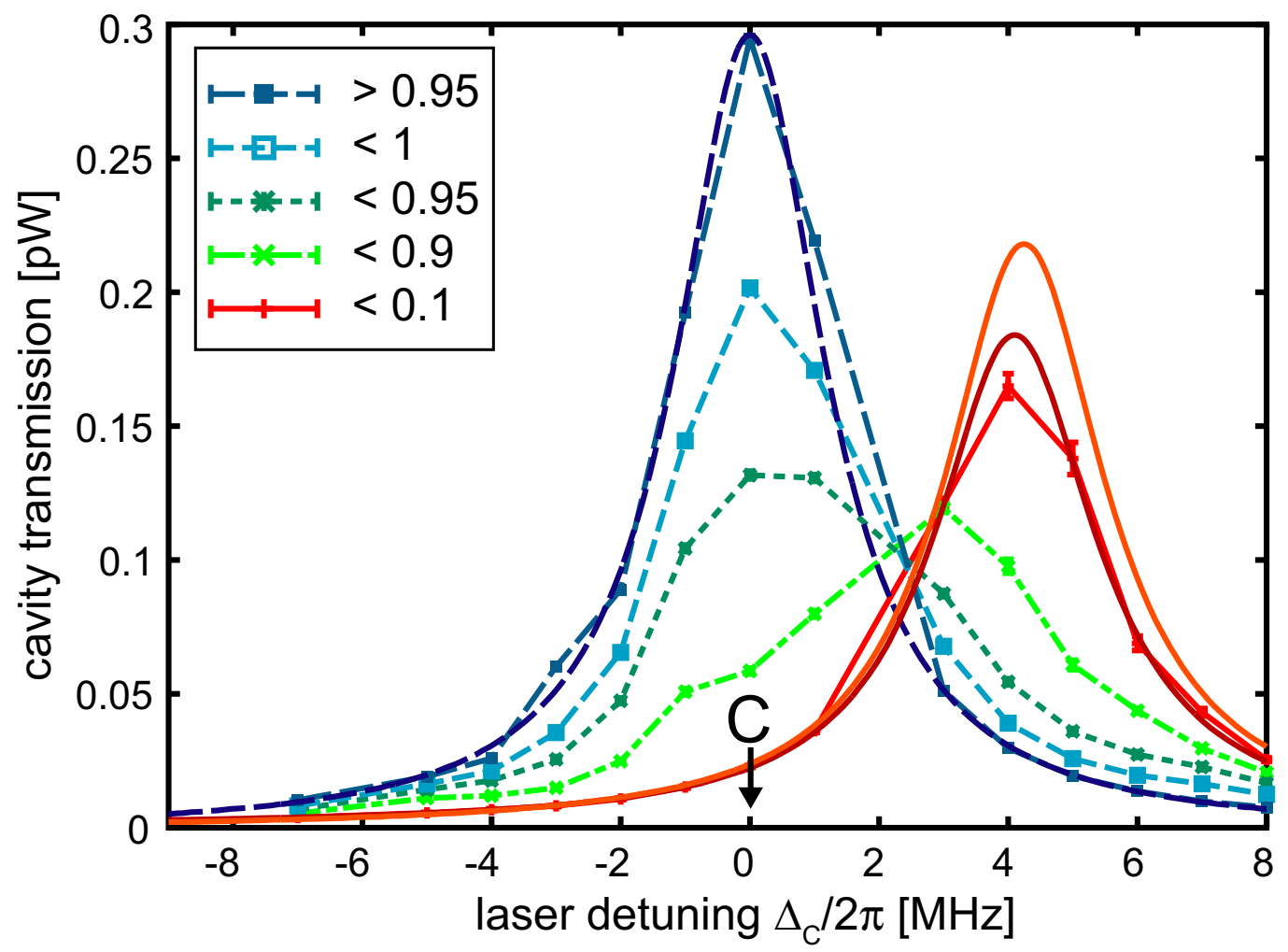

Figure 7.10: Normal mode for large detuning $\left(\Delta_{a c} / 2 \pi=-50 \mathrm{MHz}\right)$. The progressively qualified transmission on the cavity-like normal-mode peak is shown from the empty cavity (dashed blue) to the strongly-coupled system (solid red). The decreasing threshold $\xi$ qualifies for increasing coupling until the signal of the empty cavity is completely removed. The analytical expression at low excitation for an atom with fixed coupling fit the data well. Without an additional Stark shift the coupling is $g=0.87(1) \times g_{0}$ and $g=0.85(12) \times g_{0}$ for fitted Stark shift of $\Delta_{\mathrm{S}} / 2 \pi=0.3(1.4) \mathrm{MHz}$.

the loss rate on the cavity-like normal-mode peak for the bare cavity at $0 \mathrm{MHz}$ and an atom-cavity detuning of $\Delta_{a c} / 2 \pi=-50 \mathrm{MHz}$. The loss rate is defined as the inverse mean storage time and is a measure for the heating during the probe intervals. The loss peak is narrower than the transmission peak and shifted to its blue slope. This corresponds to the fact that the velocity-dependent forces cool for a red-detuned probe and heat for a blue-detuned probe, respectively.

\subsection{Cavity cooling in the blue trap}

Cavity cooling allows to restore strong coupling and compensate for heating which is unavoidable, e.g., when probing the spectrum. In the red intracavity trap an increase of the average storage times for low intensity of the near-resonant probe by about a factor of two with respect to the dark trap has been shown (88). In the dark trap, without any 


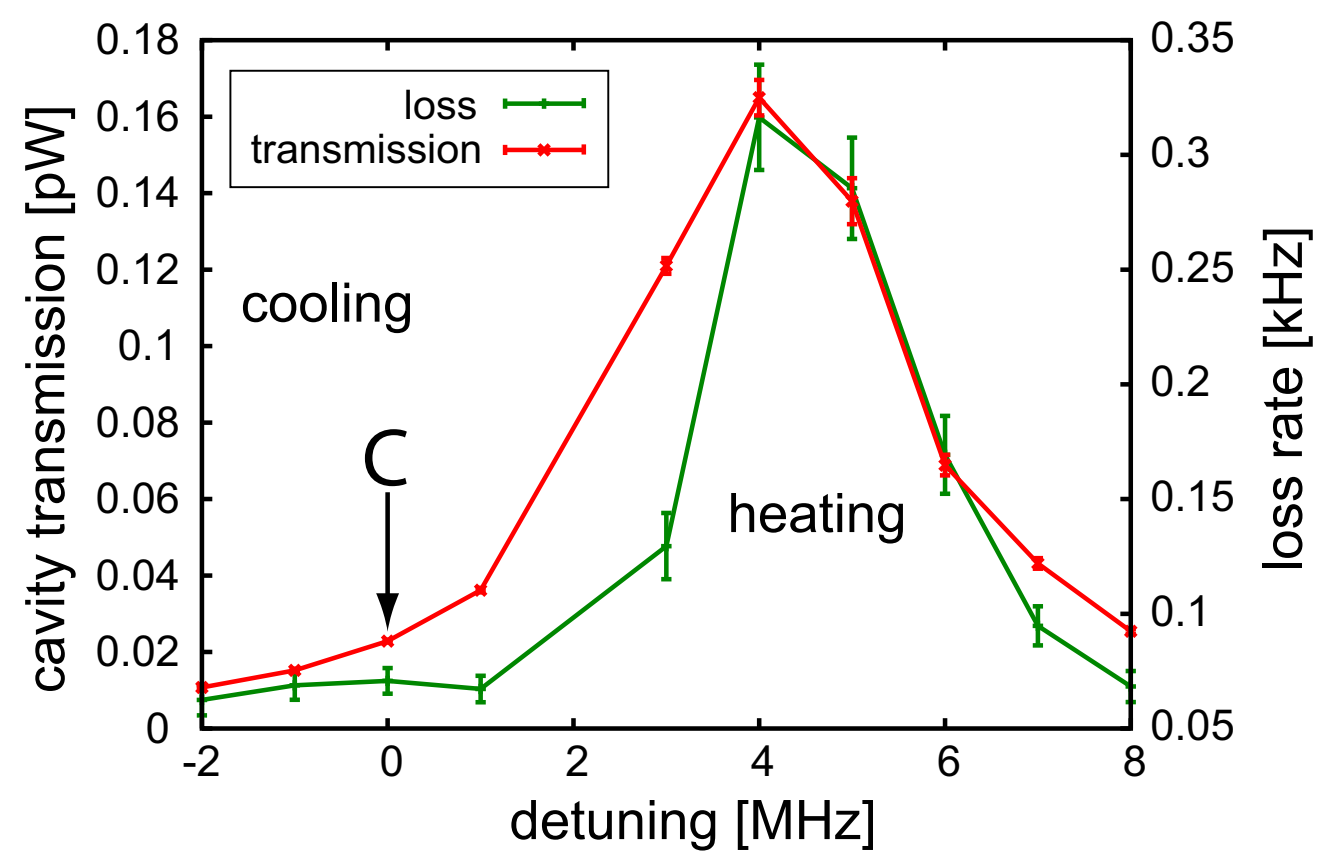

Figure 7.11: Heating and cooling on the cavity-like normal mode. The graph shows the qualified average transmission and the excess loss rate on the cavity-like normal-mode peak for the bare cavity (C) at $0 \mathrm{MHz}$ and an atom-cavity detuning of $\Delta_{a c} / 2 \pi=-50 \mathrm{MHz}$. The shift and the reduced width of the loss rate are signatures of the velocity-dependent forces which cool (heat) for a red (blue) detuned probe.

probe light, the storage time is limited by parametric heating due to the fluctuations of the trap depth at twice the trap frequency (117). At low probe intensity the axial cavity cooling compensates for this heating and leads to an increase in storage time. At the same time the excitation of the system scales with the probe power $P_{\mathrm{nr}}$ and transverse heating due to spontaneous emission recoils grows. Hence, the storage time shows a $P_{\mathrm{nr}}^{-1}$ dependence on the probe power. In the blue trap this $P_{\mathrm{nr}}^{-1}$ is also observed, see Fig. 7.12 , However, at the given parameters an increase in storage time with respect to the dark trap cannot directly be observed. The storage time at the lowest measured probe power, chosen to be compatible with continuous detection of the atom, is about the same as in the dark trap. The $P_{\mathrm{nr}}^{-1}$ dependence on the probe power is the same as observed in the red trap and supports the presence of cavity cooling. An increase in storage time is expected for even lower probe powers. A possible reason for the reduced effect of cavity cooling is the strong asymmetry in the achievable trap heights. Due to limited laser power the transverse confinement was much weaker than the axial one. The data presented in this section was taken before the laser power could be further increased by the implementation of the tapered amplifier. 


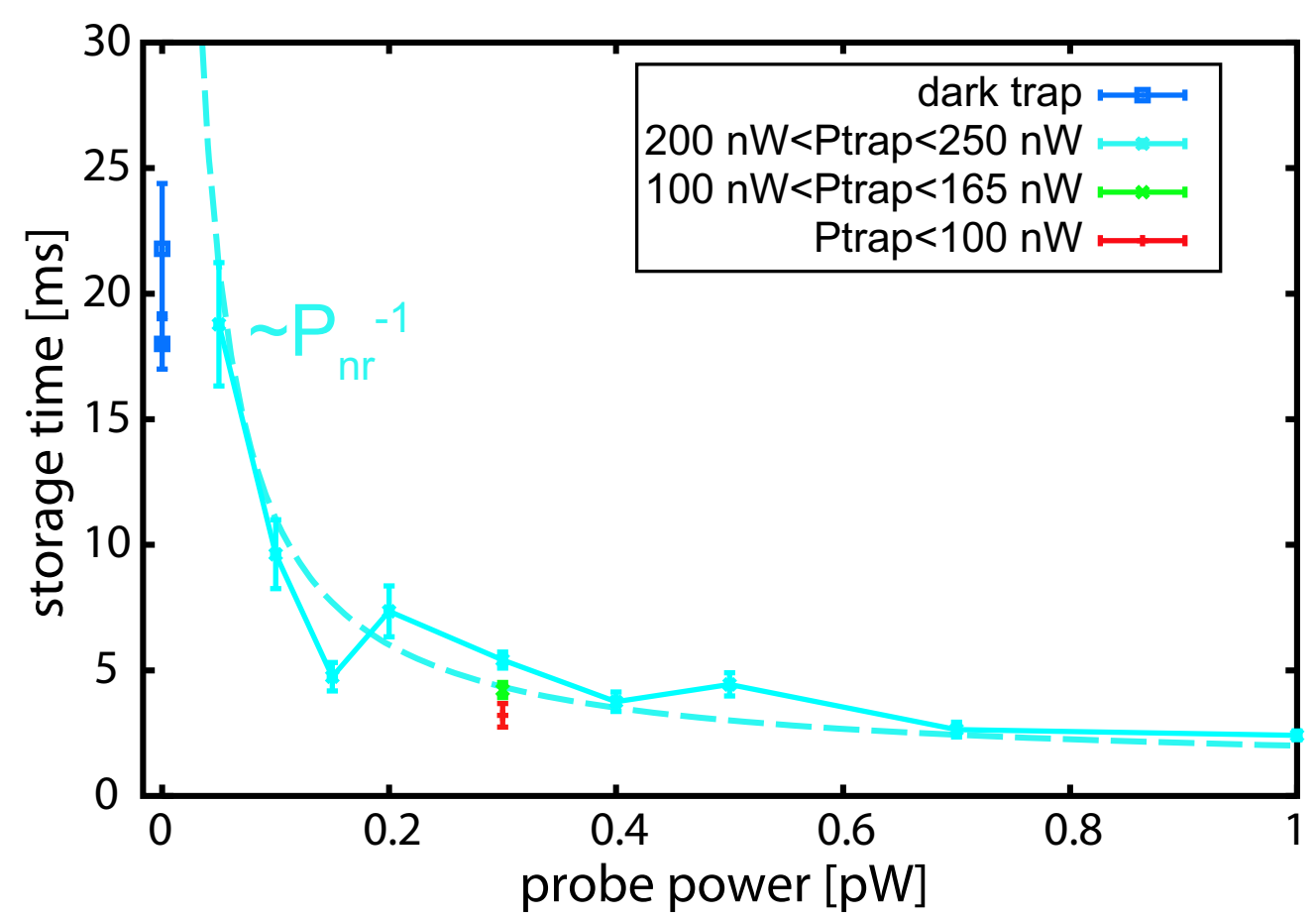

Figure 7.12: Cavity cooling in the blue trap. Dependence of the storage time with respect to the power of the near-resonant probe in the blue trap. For high probe powers the data (points) are well described by an inverse power dependence $P_{\mathrm{nr}}^{-1}$ (curve). The increase in storage time with respect to the dark trap is expected for even lower probe powers. As expected the storage time increases with the trap power shown at $0.3 \mathrm{pW}$ probe power

\subsection{Towards three-dimensional cavity cooling in the blue trap}

An increase of the storage time by several orders of magnitude can be achieved for cavity cooling in three dimensions, which requires lasers illuminating the system from the side (97). In this scheme, all probe lasers are red detuned from both normal modes, such that scattered photons are preferentially emitted with higher energy. The extra energy is extracted from the kinetic energy of the atom, which is cooled. Since effective cavity cooling requires the photons to be predominantly emitted via the cavity mode, this requires the lower dressed state to be cavity-like. An advantage of this cooling scheme is that it is effective for a strongly coupled atom at the trap center, cf. Sec. 4.1.2. In contrast, the cooling laser is resonant with the bare cavity in cooling region I (CRI, see Sec. 4.1.1), as in the experiment underlying Fig, 7.5, and cooling is achieved only for an atom close to a node and ceases for an atom cooled to the trap center.

As a first step, we have successfully captured and stored single atoms in the blue trap for the cooling region II (CRII), appropriate for 3D cavity cooling. Unfortunately the geometrical access in the current experiment prohibits well defined beams from the side. 
The edges of the cylindrical mirror substrates define two slits of about $60 \mu \mathrm{m}$ width at a distance of $7.75 \mathrm{~mm}$, incompatible with a focussed beam. Nevertheless, transverse beams have been implemented in the present setup with transmissions of about $30 \%$. This restriction can be relaxed by using coned substrates, where the cavity length shadowed by the mirror curvatures is negligible and the distance of the slits is reduced to about $1 \mathrm{~mm}$. An alternative to a near-resonant probe is a dipole field used to imprint a wavelength scale parameter modulation in the transverse directions. In addition the current vacuum chamber does not allow for direct implementation of pairs of beams in two transverse directions. A new cavity setup with coned mirrors has already been developed and should allow to implement three-dimensional cavity cooling by pairs of probe or dipole beams in the plane perpendicular to the cavity axis.

\subsection{Conclusions and prospects}

The blue trap allows to store atoms with storage times comparable to the red trap at equal trap depth. This is expected, because the dominating transverse losses due to diffusion, induced by spontaneous emission, are the same in both traps and cannot be compensated by axial cavity cooling. However, in contrast to the red trap atoms are stored close to zero intensity at the trap center, where the Stark shift vanishes and the trap height does not contribute to the effective atomic detuning. This allows for much stronger confinement, especially in the axial direction. The detection bandwidth for photon counting is limited by shot noise and cannot resolve the axial motion which is about two orders of magnitude faster than the transverse. Because the axial position cannot be directly observed nor qualified, strong axial confinement is advantageous. Losses from the dark dipole trap are induced by axial parametric heating due to field intensity fluctuations at twice the trap frequency. For strong axial confinement, this frequency exceeds the linewidth of the cavity mode of $2 \pi \times 1.4 \mathrm{MHz}$, which should lead to a suppression of field fluctuations and hence, of the heating. High trap frequencies facilitate to resolve the motional sidebands. Ground-state cavity cooling can only be achieved, when the sidebands are larger than the cavity linewidth (136). This regime should be accessible in the blue trap. Trapping field intensities of a few times $10^{6}$ photons, corresponding to trap heights on the order of $100 \mathrm{MHz}$, are limited experimentally by the available laser power. The loss-induced heat load on the mirrors when switching the dipole power cause length changes, which have to be compensated by the cavity stabilization. This limits the achievable trap depth in the red trap. In the blue trap the axial confinement and the transverse guiding need not be switched and the system can stabilize. In this case, only adding the mode to complete the transverse confinement will change the heat load.

A further advantage of this trapping scheme as compared to a red trap is that guiding slow atoms with blue fields to regions of strong coupling is not accompanied by an increase in kinetic energy. Switching the axial confinement is not necessary for trapping, such that the axial energy is not increased. Therefore, a strongly-coupled system can be prepared by guiding only and stabilized by full three-dimensional confinement. In 
contrast, in the red trap guiding is accompanied by gain in kinetic energy and cavity cooling is needed to compensate for the potential energy gained axially while switching.

Since the bare atom detuning is conserved in the blue trap, the parameters $\left(\Delta_{\mathrm{c}}, \Delta_{\mathrm{a}}\right)$ can be chosen to simultaneously allow for efficient detection as well as cooling. In particular, this enables trapping in the cooling region II (Sec. 4.1.2). At the same time the off-resonant detection of the atom can distinguish between high couplings (cf. Fig. 4.2). In this parameter regime the probe beam is red detuned with respect to both normal modes. This allows, in principle, to extend the system to include transverse cavity cooling induced by additional side beams. It was shown (137) that three-dimensional cavity cooling achieves temperatures that correspond to a dominant population in the ground state of the trapping potential.

An alternative concept is parametric cooling by applying feedback onto the transverse confinement based on the measured transmission in real time (102). In this case the blue trap has the advantage that the transverse confinement can be switched while axial confinement is preserved. The idea of the feedback can be extended by using two dimensional position information, as obtained by the atomic kaleidoscope presented in Chap. 9 .

Slow atoms collected by the blue funnels are efficiently guided to regions of strong coupling. Other atoms are repelled from the cavity. In experiments where optical cavities are investigated as single-atom detectors (131; 132; 133; 134; 135) this could lead to a more pronounced distinction and, for sufficiently well collimated atoms, to an enhanced detection efficiency. Since the passing atoms are either well coupled or expelled, the number statistics of a beam of atoms is enhanced as atoms in the tails of the mode are excluded. This corresponds to the masking invoked in the theoretical study of photon correlation spectroscopy of the two-photon excitations of the Jaynes-Cummings ladder (77).

Apart from the larger accessible parameter range in the blue trap, a major advantage for future experiments on the system is the combination of improved localization and reduced dynamical broadening due to the Stark shift. The potential of the blue trap is further demonstrated by the first signal of an excitation to the second doublet of the Jaynes-Cummings level structure presented in the next chapter. 


\section{Chapter 8}

\section{Spectroscopy of the atom-cavity system}

The vacuum-Rabi splitting for a single trapped atom was first observed with this apparatus (94) and, shortly after, on a single trapping event (138). A well resolved spectrum is a direct confirmation of strong coupling. The vacuum-Rabi splitting, as the excitation to the first doublet of the Jaynes-Cummings ladder (Fig. 2.2), is well described by semiclassical theory. It shows good agreement with the analytical theory in the low excitation or harmonic limit (Chap. 2). It can equally well be accounted for by Bloch equations as well as linear dispersion theory (51). In contrast, the spectroscopy of the higher lying states would discriminate the quantum description of the field from a semiclassical description. Inversely, these resonances are not at all included in the theory of the low excitation limit.

The interest in the spectroscopy of the second doublet of the Jaynes-Cummings ladder (Fig. 2.2) is actually present since the beginning of the field of cavity quantum electrodynamics (45). Signatures of the higher doublet frequencies in the Fourier spectrum were found in the microwave regime (78). The related effect of the photon blockade was recently observed in the optical regime (81).

In the present experiment the analysis of the spectroscopy of the second doublet was encouraged by the observation of the vacuum-Rabi splitting, which is discussed in detail in the thesis of Peter Maunz (87). In fact, the high signal to noise measurement of the anticrossing in the first doublet was also the first attempt to observe two-photon transitions with a monochromatic excitation to the second doublet. It serves as a reference to observe two-photon transitions using the excitation with two laser frequencies.

This chapter presents the spectroscopy of the strongly coupled atom-cavity system. Sec. 8.1 introduces the spectroscopy from a conceptional point of view. The measurement of the vacuum-Rabi splitting in the red trap will be reviewed in Sec. 8.2. The rest of the chapter will be devoted to the spectroscopy of the second doublet. After summarizing the considerations based on simple model systems (Sec. 8.3 the numerical simulations of the bichromatic spectroscopy will be discussed (Sec. 8.4). The development of the spectroscopy in the red intracavity dipole trap for the near-degenerate case leads the 


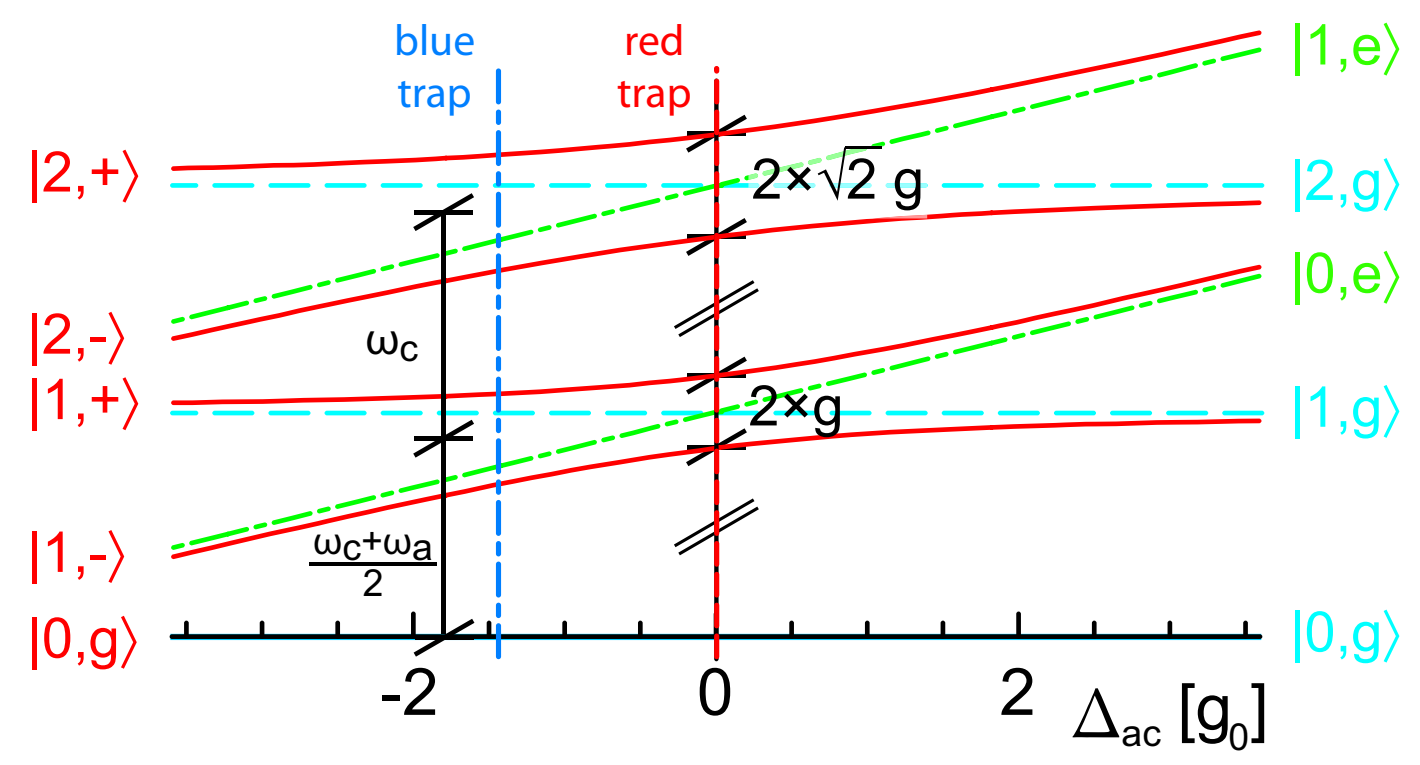

Figure 8.1: Jaynes-Cummings states. Anti-crossing of the normal modes in the first two doublets. The cavity detuning is held fixed such that the harmonic oscillator states are given by horizontal lines (dashed blue). The atomic state is varied (dashed-dotted green). The transitions between these states are shown in Fig. 8.2

way to the first signal of a two-photon resonance with detuned bichromatic excitation enabled by the blue trap (Sec. 8.5).

\subsection{Analytical concept}

The anti-crossing of the normal mode is shown in Fig. 8.1, where the ground state and the first two doublets of the Jaynes-Cummings ladder are plotted versus the atom-cavity detuning $\Delta_{a c}$. The cavity bare state is held fixed, such that the states of the harmonic oscillator are given by horizontal lines (dashed green) while the atomic state is varied (dashed-dotted blue). As discussed in Sec. 2.1, for the degenerate case $\left(\Delta_{a c}=0\right)$ the dressed states (solid red) will split by $2 \times g$ and $2 \times \sqrt{2} g$ in the first and second doublet, respectively. The coupled states are labeled by the order of the doublet $n$, i.e. number of excitations, and as lower $|n,-\rangle$ and upper state $|n,+\rangle$, respectively.

The detunings of all transitions from the ground state to the first and second manifold are plotted in Fig. 8.2. Two times two resonances occur for monochromatic excitation from the ground state to the first doublet and second doublet, respectively. A second set of four resonances arises from stepwise bichromatic excitation with two probes. In this case one laser resonantly excites a state in the first doublet and the second probes the transitions from that state to the second doublet.

In Fig. 8.2 the bare atomic resonance $|1, g\rangle$, is shown for reference (blue dasheddotted line). The red lines (1), (2) are the transitions to the first doublet $|0, g\rangle \rightarrow|1, \mp\rangle$. 


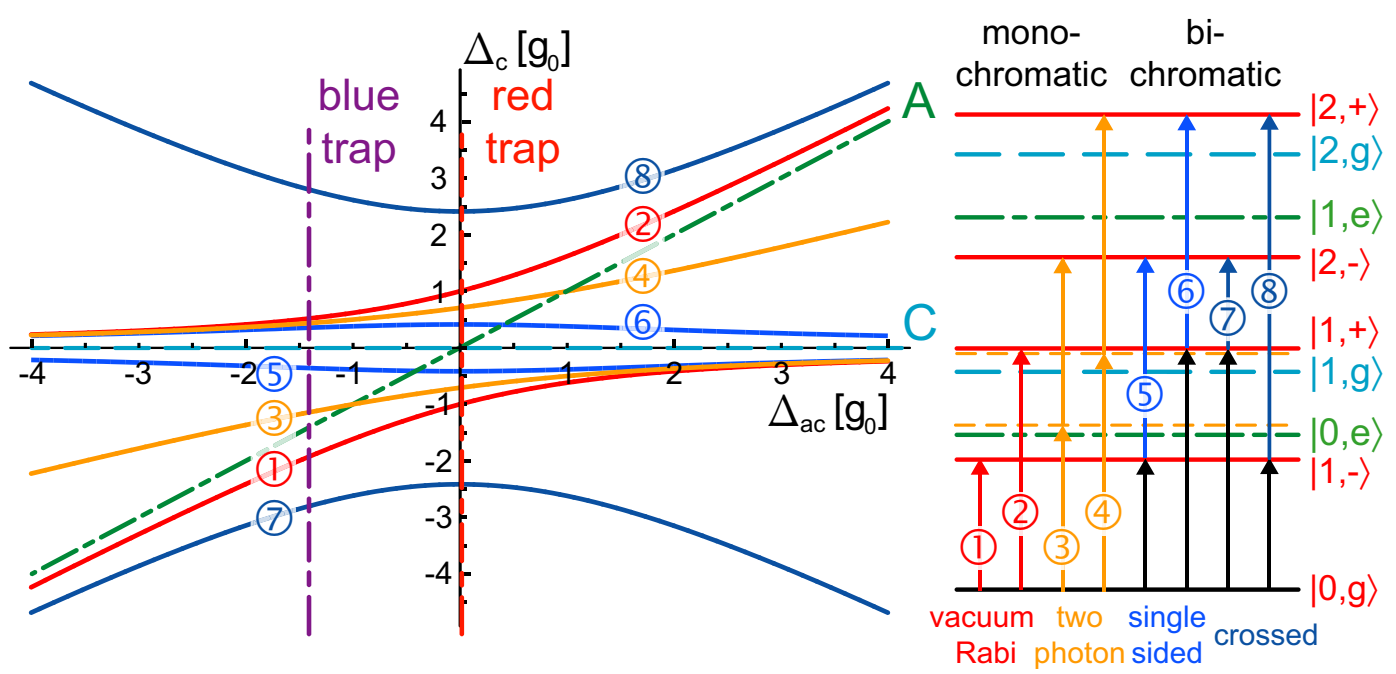

Figure 8.2: Transitions up to the second doublet of the Jaynes-Cummings ladder: The two transitions to the first excited doublet are shown in red (1), (2) and the monochromatic transitions to the second doublet in orange (3), (4). The step-wise bichromatic excitations divide into two groups: the single sided (blue, (5), (6)) and the crossed (dark blue, (7), (8))). In detuned case $\Delta_{a c} \neq 0$ the transitions on one side of the cavity resonance are well separated. This is due to the difference in the distance between the ground state and first doublet and first and second doublet, cf. Fig. 8.1. The near-resonant case of the red trap and the detuned case in the blue trap are indicated by vertical lines corresponding to a scan in laser frequency.

They represent the anticrossing of the vacuum-Rabi spectrum. Two photons of the same detuning (orange, (3), (4) excite the system to the second doublet $|0, g\rangle \Rightarrow|2, \mp\rangle$ via a virtual level close to the first doublet, i.e. at $\sqrt{g} / 2$ for $\Delta_{a c}=0$. A second group of excitations to the second doublet are bichromatic stepwise excitations, assuming that a second frequency is exciting one of the first-order normal modes resonantly. These can be single sided via lower state to the lower state $|0, g\rangle \rightarrow|1,-\rangle \rightarrow|2,-\rangle$ (5)) or upper to upper state $|0, g\rangle \rightarrow|1,+\rangle \rightarrow|2,+\rangle$ (6) or crossed transitions via the upper to the lower states $|0, g\rangle \rightarrow|1,+\rangle \rightarrow|2,-\rangle$ (7) or vice versa $|0, g\rangle \rightarrow|1,-\rangle \rightarrow|2,+\rangle$ (8). The single-sided transitions lay within the two monochromatic two-photon transitions. The crossed resonances are expected to be well separated at the outside of the normal-modes at $\pm(1+\sqrt{2}) \times g$ for $\Delta_{a c}=0$.

The natural way to probe the system in the experiment is to vary the laser while keeping the atom-cavity detuning fixed. This corresponds to a frequency scan along a vertical line in Fig. 8.2 at a given $\Delta_{a c}$. Note that in the detuned case $\Delta_{a c} \neq 0$ the distance between the ground state and first doublet, given by $\left(\Delta_{\mathrm{a}}+\Delta_{\mathrm{c}}\right) / 2$, differs from the separation of the first to second doublet which is $\Delta_{\mathrm{c}}$ (cf. Fig. 8.1). It is clear from Fig. 8.2 that by choosing an atom-cavity detuning different from zero $\Delta_{a c}>0\left(\Delta_{a c}<0\right)$ one set of transitions above (below) the bare cavity will split by a larger amount and hence, the resonances to the second doublet are better resolved from transitions to the first doublet. This is particularly important in the experiment where the resonances are 

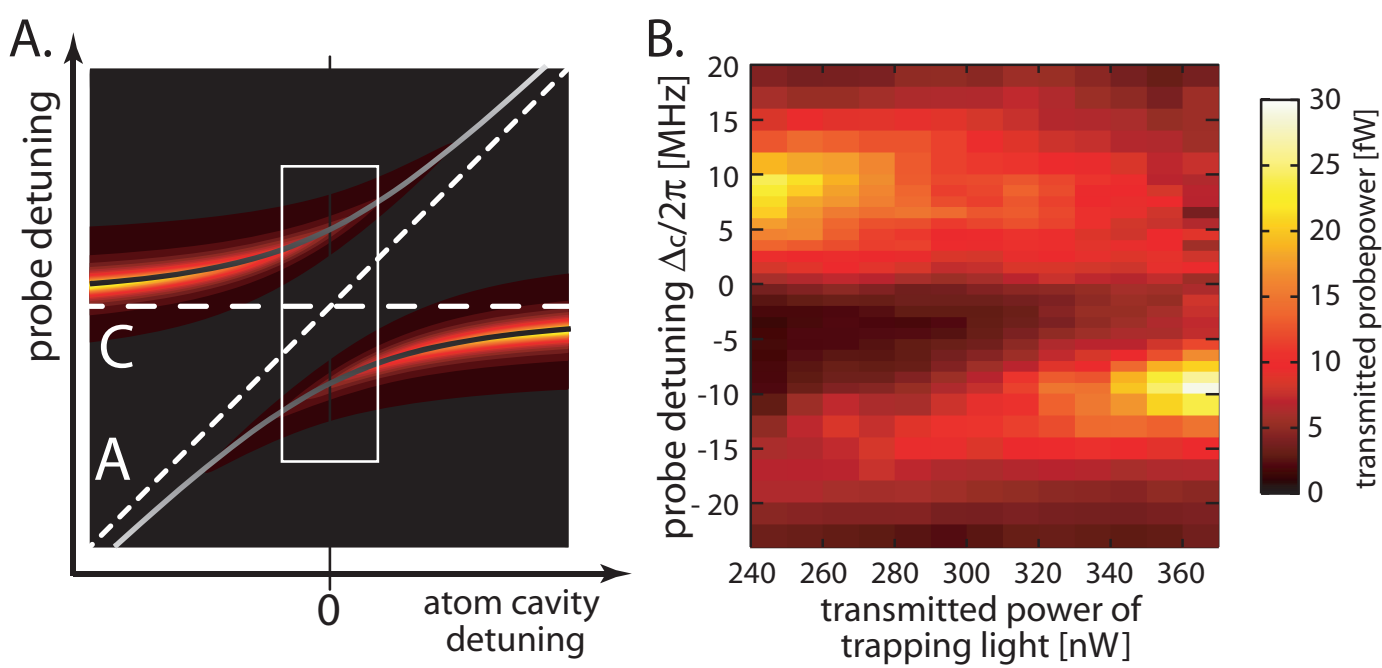

Figure 8.3: Anticrossing in the vacuum-Rabi splitting observed in the cavity transmission. The atomic state $(\mathrm{A})$ is tuned through resonance with the bare cavity state $(\mathrm{C})$ by varying the Stark shift induced by the depth of the red trap. A. Theoretical transmission: because the system is both excited and detected via the cavity mode, the cavity-like state is more pronounced. B. Measured transmission for intervals of qualified good coupling for the parameter region of the white box in $\mathbf{A}$.

broadened by the dynamics of the atom in the mode. On the other hand, an increasing atom-cavity detuning $\Delta_{a c}$ reduces the mixing angle between the bare states such that the dressed states are dominated by the contribution of one of the bare states (see 2.8). The strengths of the transitions between the levels is determined by the overlap between the involved states with respect to the way the system is excited as well as detected. Especially in the case of a stepwise two-photon excitation in the detuned case, the different transition strength can be used to optimize the excitation path, as will be discussed in more detail in Sec. 8.5.2.

For cavity QED experiments in the optical regime the continuous detection is realized by the transmission through the cavity mirror. In the current experiment the system is excited by a probe laser via the cavity mirrors. Both processes address the cavity part of the coupled states, thus the normal-mode splitting shows a clear asymmetry in the peak heights, which interchanges when scanning through the anticrossing (cf. Fig. 8.3). In the limit of large detuning the cavity-like state $(\mathrm{C})$ will dominate the spectrum. In the degenerate case both normal modes are observed with equal heights, since their contributions to the dressed states are equal.

\subsection{Normal-mode splitting}

The measurement and analysis of the normal-mode splitting in the red intracavity dipole trap (Fig. 8.4 is presented in detail in the thesis of Peter Maunz (87). The picture 


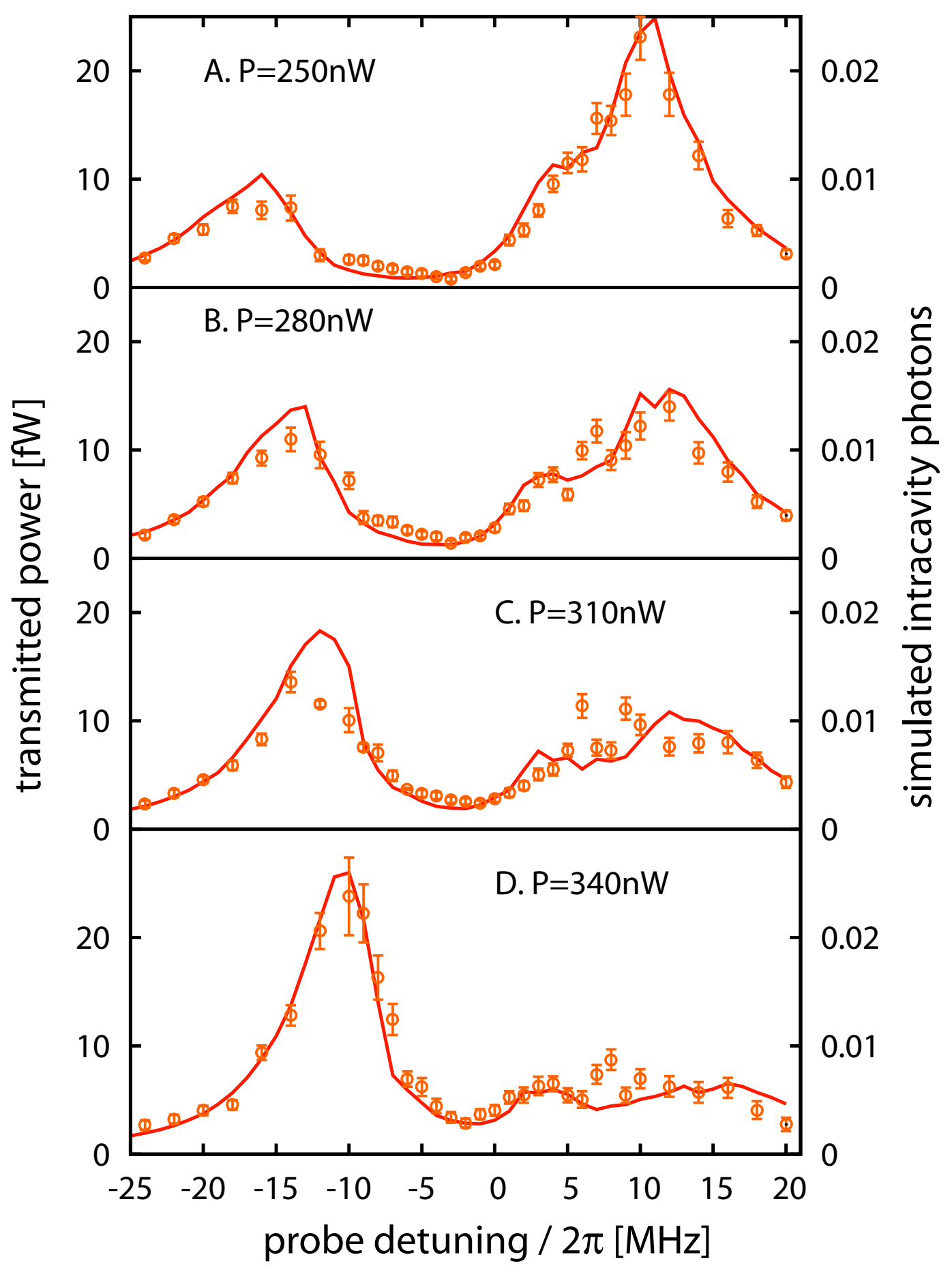

Figure 8.4: Transmission spectra of the normal-mode splitting observed in the cavity transmission for qualified strong coupling (points). The atom-cavity detuning $\Delta_{a c}$ is scanned through the anticrossing by variation of the Stark shift $\Delta_{\mathrm{S}}$ induced by the red trap. The Stark shift $\Delta_{\mathrm{S}}$ effectively shifts the bare state of the atom at $-35 \mathrm{MHz}$ close to resonance with the empty cavity at $0 \mathrm{MHz}$. A scan of the probe detuning corresponds to a vertical line in Fig. 8.3. The increasing $\Delta_{a c}$ in the consecutive scans is given by the power of the trap field $\mathrm{P}$. The probe power corresponds to 0.3 photons in the empty cavity or about $300 \mathrm{fW}$ transmission. The solid lines are the result of a Monte Carlo simulation based on the semiclassical theory in the low excitation limit. 


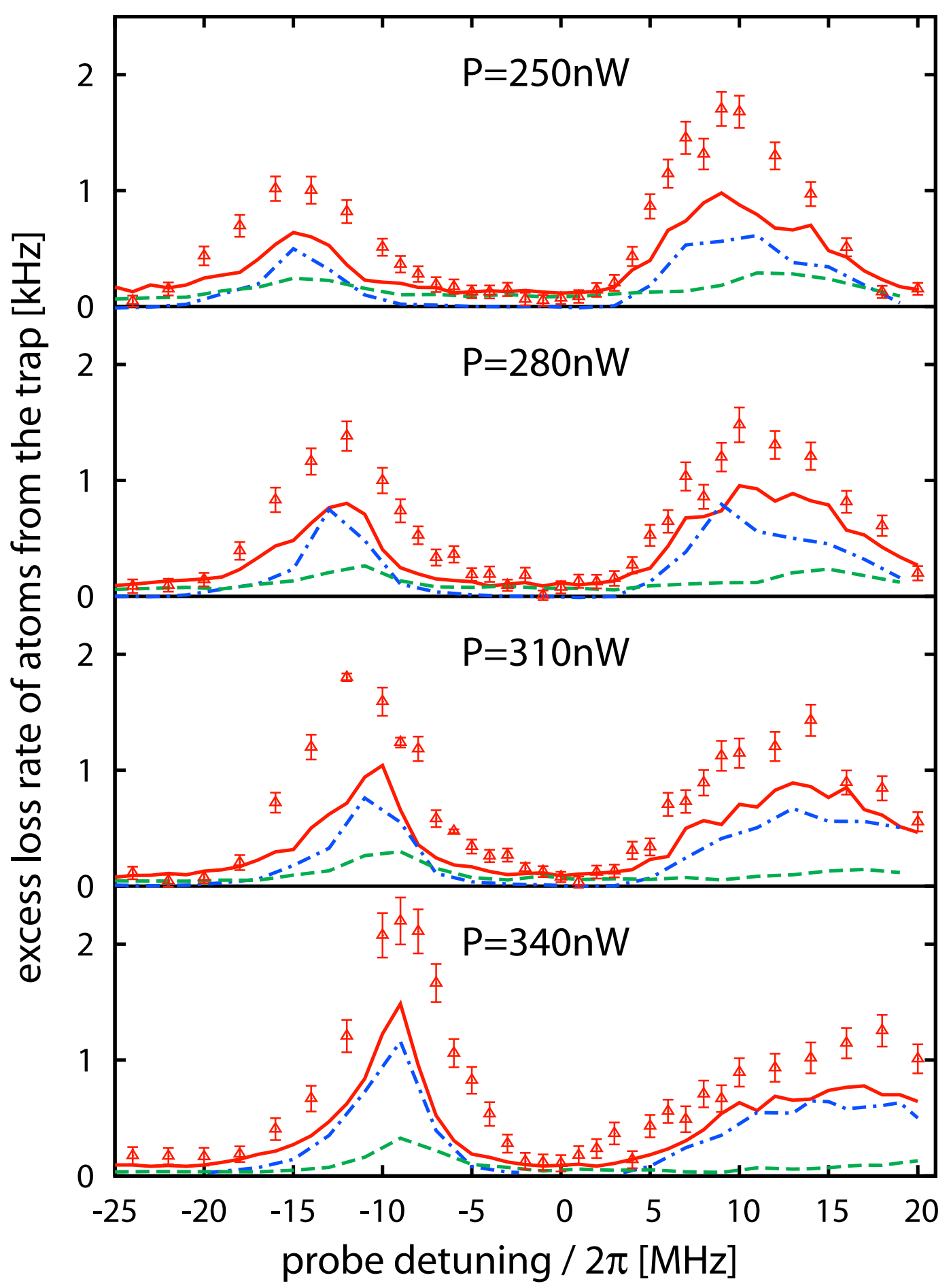

Figure 8.5: Spectra of the normal-mode splitting observed in the excess loss rate induced by the probing at different detunings (parameters are the same as in Fig. 8.4). The lines are the result of Monte Carlo simulations: The diffusion due to spontaneous emission by the atom (dashed) cannot account for the losses near the normal modes. The pronounced spectrum is a signature of the large diffusion due to dipole-force fluctuations in the atom-cavity system (dotted). The simulation including both contributions is shown as solid lines. 
shows the average transmission (points) of the cavity for probe intervals of good qualified coupling between atom and cavity mode. The spectra are taken close to the resonant case $\Delta_{a c}=0 \mathrm{MHz}$ at the center of the anticrossing (see Fig. 8.3, white box). To effectively scan the atomic detuning through the resonance with the cavity at $\Delta_{\mathrm{c}}=0 \mathrm{MHz}$, the Stark shift at the trap center is varied by changing the depth of the red intracavity dipole trap. The experimental difficulty in observing the spectra is to eliminate the problem of variations of the coupling and Stark shift due to the atomic motion inside the standing wave mode. A well resolved near-resonant normal-mode spectrum proves reliable strong coupling. Since the empty cavity transmission with an amplitude of $0.3 \mathrm{pW}$ is more than an order of magnitude larger than on the normal-modes resonances of the coupled system, any residual signal of a weakly coupled atom results in a large spurious contribution to the spectrum. Strong velocity-dependent forces as well as strong diffusion are present in the vicinity of the normal modes and drive the spatial distribution of the atom. To compensate for the heating, probe intervals are embedded in-between cooling intervals. The transmission during these cooling intervals at the same time serves as an independent qualification. The spectra show good agreement with the Monte Carlo simulations (solid lines). A pronounced structure is also observed in the corresponding loss spectra shown in Fig. 8.5. The excess losses on the normal modes cannot be explained by heating due to spontaneous emission, but are dominated by the enhanced dipole force fluctuations in the atom-cavity system (139).

\subsection{Theoretical analysis of the two-photon spectroscopy}

The theoretical analysis of the two-photon spectroscopy was developed together with Niels Syassen and details can be found in his diploma thesis (140). Starting from analytical studies of simple lossless model systems, finally, the open atom-cavity system was studied by numerical simulation of the master equation with two probe frequencies.

\subsubsection{Model systems}

Before considering the spectroscopy of the Jaynes-Cummings ladder, some basic insight can be obtained from the analysis of simple model systems. The simplest system that allows for two-photon excitation is the three-level system. Generally, systems without dissipation do not reach a steady state such that physical quantities, like the populations, show oscillations. These oscillations on the order of the couplings and detunings are assumed to be much faster than the integration time of the detection. Hence, relevant mean values of physical quantities are established by time-averaging over a sufficiently long interval.

The equidistant lossless three-level system, depicted in Fig. 8.6, A., can be solved analytically. For zero detuning $\delta=0$ the system undergoes full population oscillations between the ground state $|g\rangle$ and the final state $|f\rangle$. The population of the intermediate state $|e\rangle$ oscillates out of phase at twice the frequency and is zero if either of the others is 1 . If the detuning is increased to twice the pump strength $(\delta=2 \eta)$, all populations 

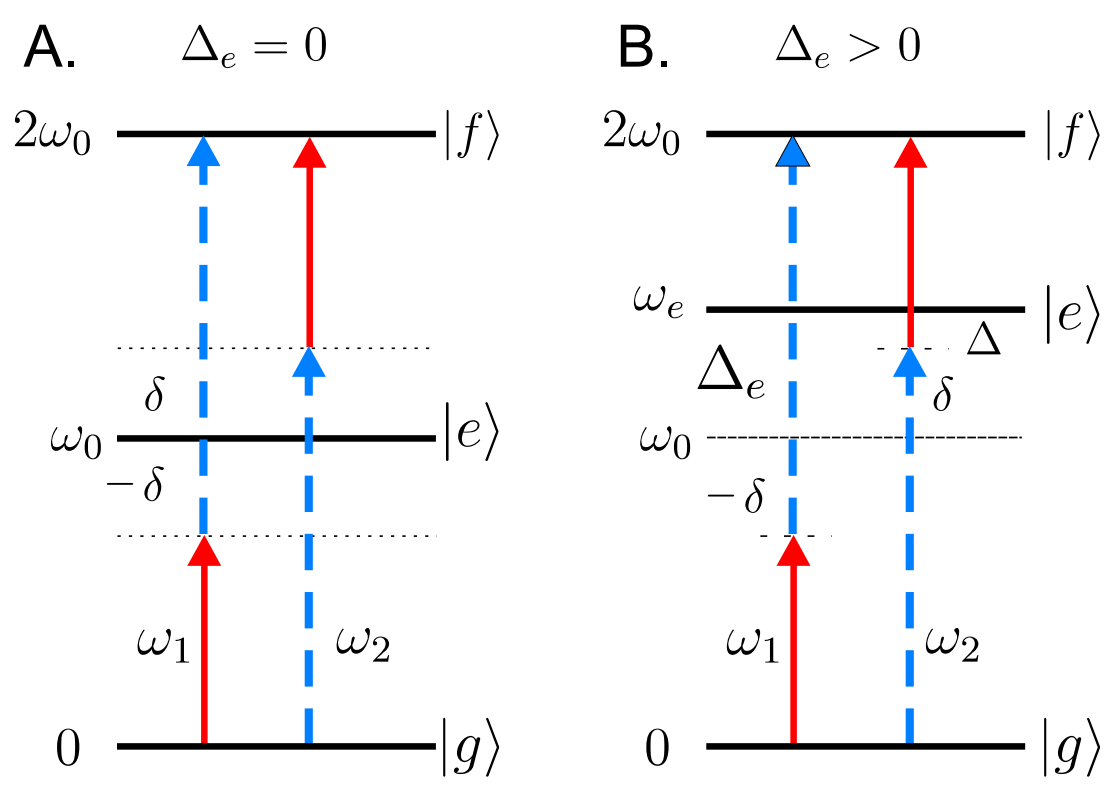

Figure 8.6: Lossless three-level system. A. In the equidistant three-level system a bichromatic excitation is equivalent to an oscillating Rabi frequency. B. In a non-equidistant three-level system one excitation path can be preferred.

oscillate at the same frequency such that the full inversion cannot be reached. Instead, the population $P_{f}$ is smaller than the population in $P_{e}$. In the limit of large detuning and low excitation the populations are small and $P_{f} \propto P_{e}^{2}$ scales quadratically with $P_{e}$. To achieve a population of $P_{f}$ exceeding that of $P_{e}$, it is therefore necessary to work in the limit of low detuning $(\delta \lesssim \eta)$.

In contrast, the non-equidistant lossless three-level system (Fig. 8.6, B.) shows a true two-photon transition when the detuning from the intermediate level $|e\rangle$ is larger than the pump strength $\eta$ (Fig. 8.7). The population of the intermediate level $|e\rangle$ drops as $P_{e} \propto \eta^{2} / \Delta^{2}$ corresponding to off-resonant excitation. The population in the ground and final state converge to $P_{g}, P_{f} \rightarrow 0.5$. If losses are included, a numerical simulation of the master equation shows that to reach significant populations, the driving $\eta$ has to be larger than the losses $\eta>\left(\gamma_{e}, \gamma_{f}\right)$. In this case the intermediate state is populated by the decays from the final state. A final state population exceeding the one of the intermediate state $P_{f}>P_{e}$ is possible for strong driving if $\gamma_{f}<\gamma_{e}$, i.e. the final state decay rate is smaller than the decay rate of the intermediate state.

\subsubsection{Spectroscopy of the atom-cavity system}

The atom-cavity system is well described by the semiclassical analytical theory in the low-excitation limit. In this limit the theory includes the normal modes as excitations to the first doublet, but does not include excitation to the second doublet. Hence, the analytical solution in the low-excitation limit is inadequate for the study of the 

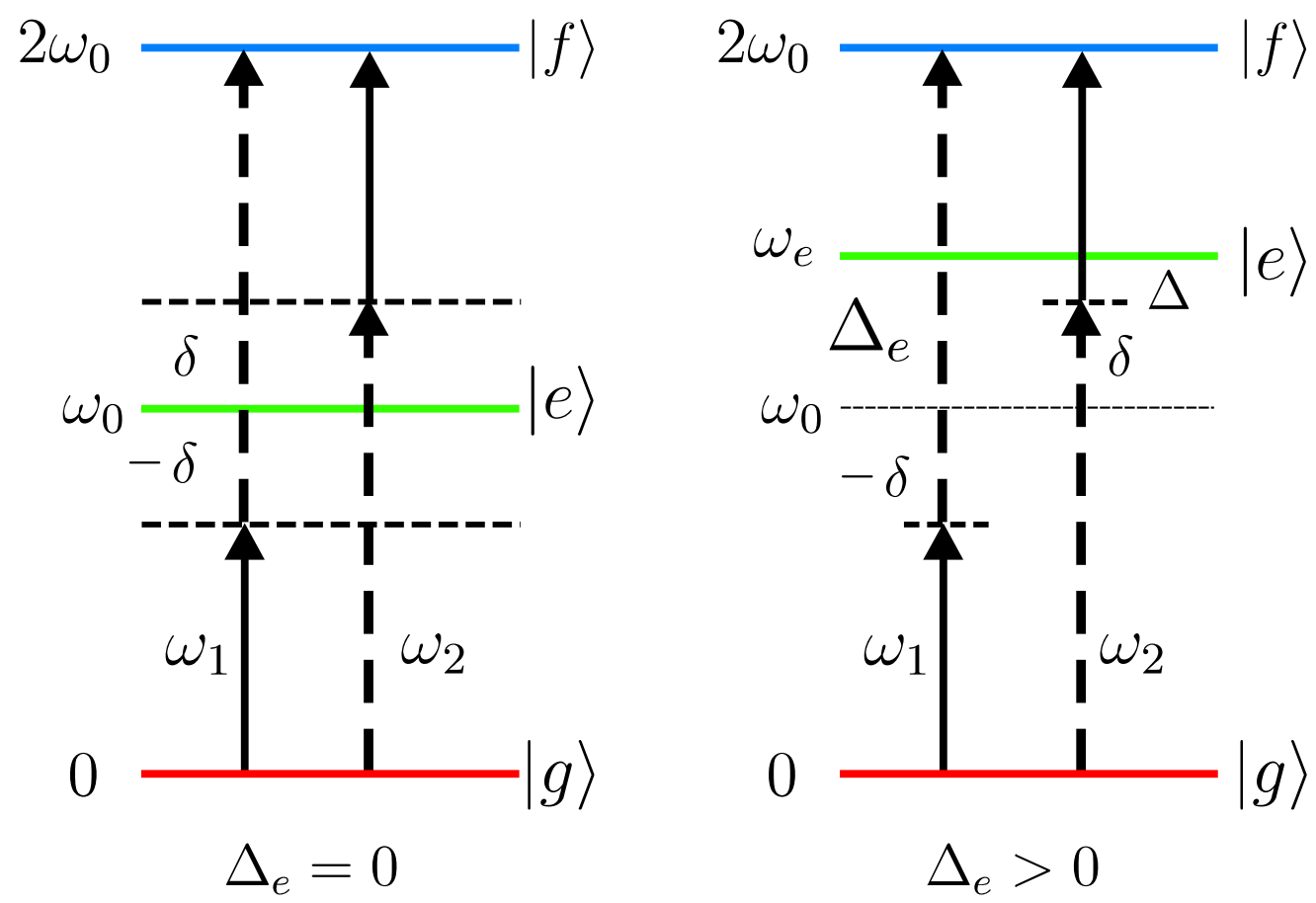

Figure 8.7: Populations in the lossless non-equidistant three-level system In a nonequidistant three-level system the population of the final level $|f\rangle$ can exceed that of the intermediate one $|e\rangle$.

two-photon excitations. The analysis of the two-photon excitations has to include at least the states up to the second doublet. Depending on the experimental parameters in a Jaynes-Cummings level system, possible analogies to three-level systems allow to conclude some basic prerequisites for the two-photon spectroscopy. One conclusion is that for the near-degenerate case $\left(\Delta_{a c} \approx 0\right)$, the two probe fields should be of about equal strength. Quite generally the two-photon transition should be resonant with the final state and the detuning to an intermediate state should be on the order of the pump strength to achieve a noticeable population in the second manifold.

The system is excited whenever (a combination of) the detuning(s) of the probe beam(s) match a resonance of the system. The system can then relax either by spontaneous emission from the atom or by photons lost from the cavity mode. In most experiments the directed photons emitted from the cavity are detected. The linewidths of the various transitions are given by combinations of decay rates for the atomic dipole $\gamma$ and cavity field $\kappa$. They can be calculated from the master equation in the secular approximation which neglects coherences (141). When the system is excited via a two-photon transition, it will relax by a consecutive emission of two photons of different frequencies on the timescale of the lifetime of the intermediate level. In principle, efficient frequency resolved detection would be possible with heterodyne detection, not implemented in the experiment. An alternative approach to discriminating two-photon events is photon 
correlation spectroscopy, where enhanced two-photon correlations are expected in the auto-correlation function $g^{(2)}\left(\omega_{1}, \omega_{2}, \tau\right)$ of the detected photon stream (77). Since the detected photons are emitted by the cavity, the signal is proportional to the probability of a contribution of more than one photons in the cavity mode given by $\left\langle a^{\dagger} a^{\dagger} a a\right\rangle$.

\subsection{Numerical simulations}

To analyze the possibility to observe the two-photon signal in the transmission or by correlation spectroscopy, numerical simulations of the master equation were performed. Truncating the Hilbert space at the second excited manifold keeps the complexity at a minimum, while the sought-after qualitative effects are preserved. Saturation effects will be negligible at low excitation and overestimated for higher excitation.

\subsubsection{Algorithm}

The master equation (2.27) up to the $m^{\text {th }}$ doublet can be written as a set of $n^{2}(n=$ $2 \times m+1$ ) coupled linear differential equations, for the complex valued populations $\rho_{k k}$ and the coherences $\rho_{k l}=\rho_{l k}^{*}(l \neq k)$. Because the density matrix $\rho$ is hermitian the system can be reduced to a matrix equation for a real-valued vector $\mathbf{R} \in \mathbb{R}^{n^{2}}$ : $\dot{\mathbf{R}}=M \mathbf{R}$. The product $M \mathbf{R}$ is explicitly evaluated to eliminate vanishing entries and reduce the computation time. The resultant ordinary differential equation is solved using an explicit Runge-Kutta method with variable step size (142), for sets of parameters $\left(\eta_{1}, \eta_{2}, \Delta_{1}, \Delta_{2}, g, \kappa, \gamma, \Delta_{a c}\right)$ and initial conditions $\mathbf{R}(0)=\mathbf{R}_{0}$.

The pump term now includes two different lasers with detunings $\Delta_{1}=\omega_{1}-\omega_{a}$ and $\Delta_{2}=\omega_{2}-\omega_{a}$ :

$$
H_{\mathrm{P}}(t)=\left(\eta_{1} e^{-i \Delta_{1} t}+\eta_{2} e^{-i \Delta_{2} t}\right) a^{\dagger}+h . c . .
$$

Due to the explicit time dependence of $H_{\mathrm{P}}(t)$, the asymptotic solution to the master equation will oscillate with the difference frequency $\Delta=\left|\Delta_{1}-\Delta_{2}\right|$. The dc-component $\rho_{0}$ of the asymptotic solution of the density matrix $\rho(t)$ can be found using a Bloch function expansion $\rho(t)=\sum_{k=-\infty}^{\infty} \rho_{k}(t) e^{-i k \Delta}$ in frequency components of multiples of $\Delta$ (77).

Here, the quasi-stationary density matrix $\bar{\rho}$ is calculated as the mean value of a adequately long sample of the oscillating asymptotic solution instead. This is practical for sufficiently large $\Delta \simeq(\kappa, \gamma)$. For $\Delta=0$ the two fields can effectively be treated as one field with double the field strength and hence, the asymptotic solution is stationary in the rotating frame.

Once the quasi stationary solution $\bar{\rho}$ is known, expectation values of operators $O$ are calculated by

$$
\langle O\rangle_{s s}=\lim _{t \rightarrow \infty} \operatorname{Tr}(O \rho(t)) \approx \operatorname{Tr}(O \bar{\rho}) .
$$

The result of the simulations was verified on basic configurations and by comparison to other results (77). An example of bichromatic spectroscopy of the atom-cavity system will be presented in the next subsection. 


\subsubsection{Bichromatic spectroscopy of the Jaynes-Cummings ladder}

Numerical simulations of the spectroscopy with two probes were performed in the degenerate case $\left(\Delta_{a c}=0\right)$ for a fixed atom, as appropriate for measurements in the red trap. Figure 8.8 shows the spectra of the populations of the ground state and the first two doublets with respect to one of the probe frequencies, while the other is fixed. The resonances in the populations can be assigned to different transitions between the states. Because the fixed probe is close to resonance with the state $|1,+\rangle$, this state shows a constant background excitation. Moreover, a small contribution of monochromatic twophoton excitation is observed for the $|2,+\rangle$ in the second doublet. The populations of the first doublet show the vacuum-Rabi resonances, when the second probe comes into resonance with the corresponding state. The populations of the states in the second doublet show resonances for the four different mono- and bichromatic excitation paths. In the present experiment the arrival times of the transmitted photons are recorded. The photon stream can hence either be evaluated for the transmission spectrum $\left\langle a^{\dagger} a\right\rangle$ or two-photon correlations $\left\langle a^{\dagger} a^{\dagger} a a\right\rangle$. Scanning both probe fields, the strong resonances are expected in the transmission whenever one of the probes is resonant with a onephoton transition. In the simulation presented in Fig. 8.8, the strength of the probes is low, such that saturation effects do not play a significant role. The populations in the second doublet scale with the square of the populations in the first doublet and are therefore much smaller. Hence, the transmission is dominated by the excitations to the first doublet, which completely mask the contributions of the second doublet. Correlation spectroscopy extracts the two-photon signal from the transmission. The nonlinearity of the Jaynes-Cummings ladder is observed in a shift of the resonances towards smaller coupling.

Experimentally, the observation of the two-photon resonance for the near-degenerate case using photon counting is difficult because the transmission is completely dominated by the first-order excitation which is moreover broadened by dynamical effects. Correlation spectroscopy is hampered by the quadratic effect of the limited detection efficiency on the detected coincidences. Simulations for higher probe powers show an enhancement of the two-photon contribution due to saturation effects. However, to prevent perturbing effects of the atomic motion driven by large cavity heating the experimental studies presented in the following are restricted to the low excitation limit. As outlined in the analytical discussion in Sec. 8.1 the key to the measurement in the blue trap is to separate the two-photon signal from the vacuum-Rabi spectrum by using a non-zero atom-cavity detuning.

\subsection{Experimental two-photon spectra}

First experimental measurements on two-photon excitations were done in the red trap where the bichromatic spectroscopy with two probe fields was implemented. 

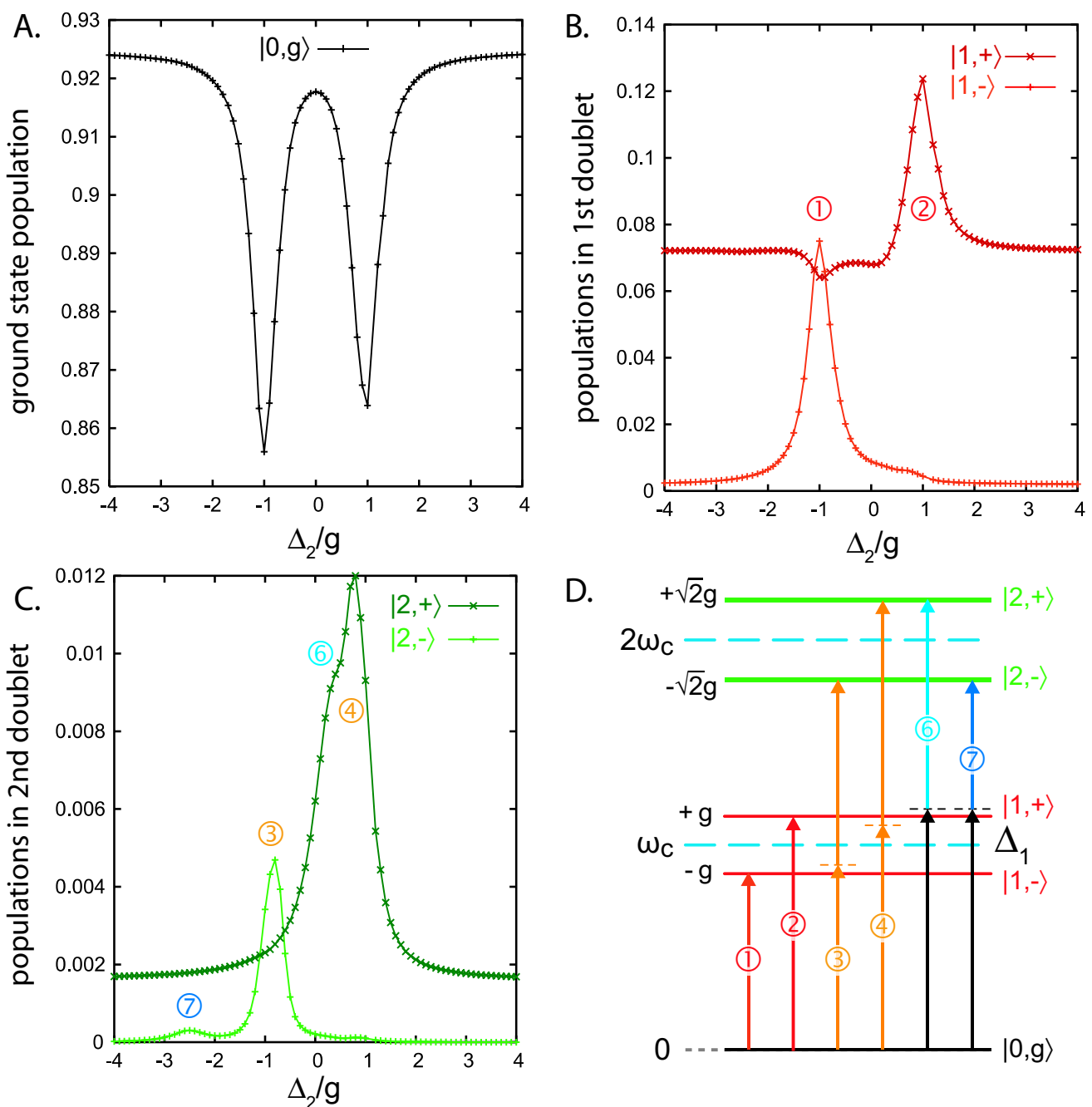

D.
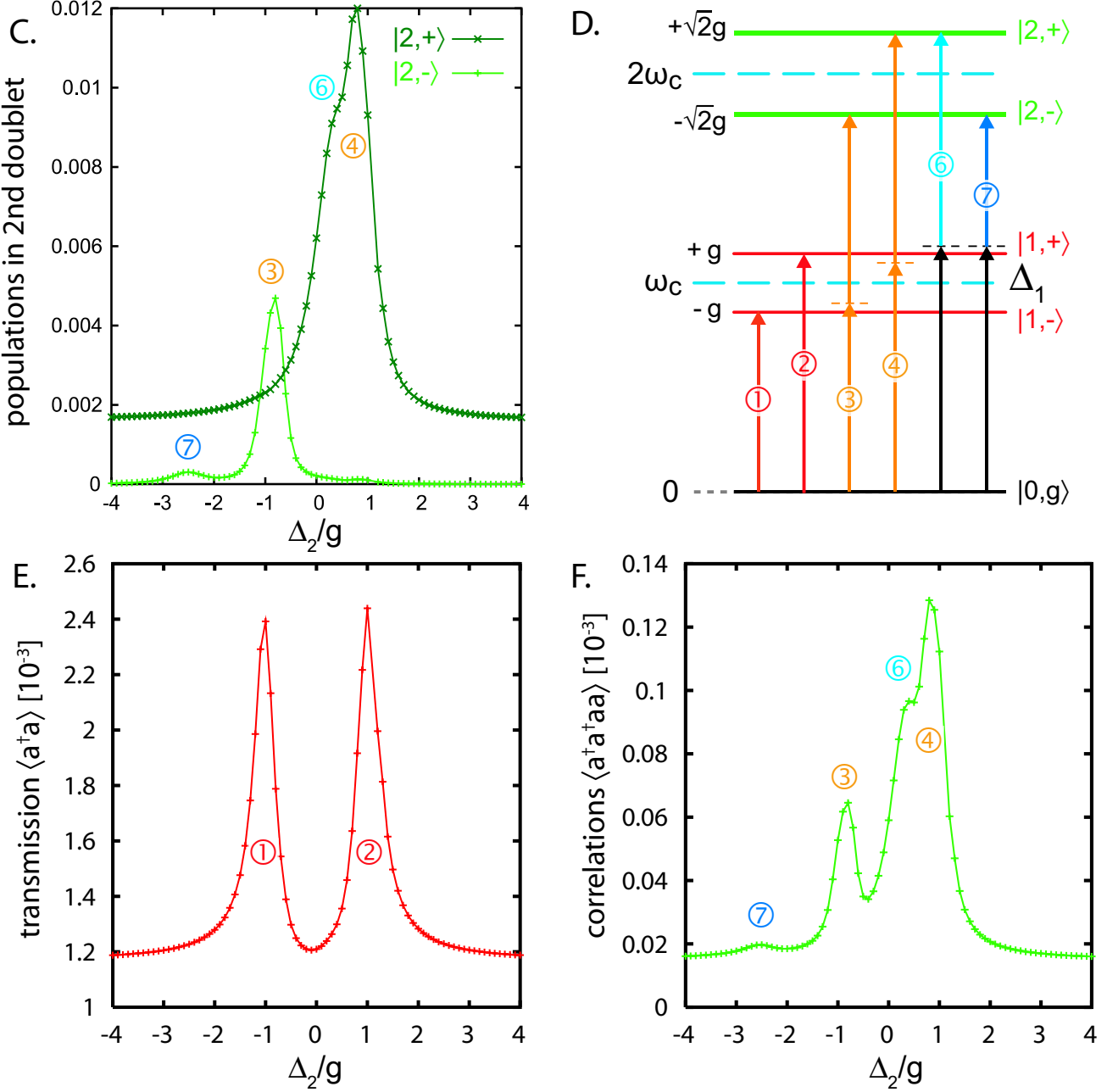

Figure 8.8: Simulation of bichromatic spectroscopy of the Jaynes-Cummings ladder. Population in the ground state (A.) and the first (B.) and second (C.) doublet for bichromatic excitation. One probe detuning is fixed close to the transition to $|1,+\rangle$, the second probe is scanned. The observed resonances can be attributed to the different possible transitions (D.). E. Cavity transmission and $\mathbf{F}$. two-photon correlation spectrum. The parameters are $\Delta_{a c}=$ $0, \Delta_{1}=\frac{11}{10} g_{0}, \eta_{1}=\eta_{2}=\frac{1}{3} \kappa$. 


\subsubsection{Two-photon spectrum in the red trap}

The transmission spectrum of the qualified strongly-coupled atom-cavity system in the red intracavity dipole trap is depicted in Fig. 8.9. For comparison the one laser normalmode spectrum at the same trap depth is plotted (cf. Fig. 8.4 c). For the dipole trap power of $P=310 \mathrm{nW}$ the Stark shift largely compensates the bare atom detuning of $\Delta_{a c} / 2 \pi=-35 \mathrm{MHz}$ such that the atomic state is close to resonance with the empty cavity at $0 \mathrm{MHz}$. A coupling of $g / 2 \pi=12 \mathrm{MHz}$ and atom-cavity detuning $\Delta_{a c}=2 \pi \times$ $-3.8 \mathrm{MHz}$ are obtained by Gaussian fits to the experimental spectrum and confirmed by comparison with Monte Carlo simulations $(g=2 \pi \times 13 \mathrm{MHz})$. The single frequency two-photon transitions at $\Delta_{1} \approx \pm(\sqrt{2}-1) g \approx \pm 5 \mathrm{MHz}$ with respect to the bare states, should be observable as small bumps on the inner slopes of the normal-mode peaks. No signatures of these resonances are discernable from the noise, which is in agreement with the simulations at the low excitation level of 0.3 photons in the empty cavity (i.). Unfortunately, the experimental two-photon correlation signal scales quadratically with the small count rate given by the limited detection efficiency and hence does not show a conclusive signal.

As discussed before, a larger separation from the first-order resonances can be realized for the crossed transitions using two probes. The green spectrum Fig. 8.9 is taken with the first probe at a fixed detuning of $\Delta_{1}=2 \pi \times 11 \mathrm{MHz} \approx g$, exciting the upper normal mode $(|0, g\rangle \rightarrow|1,+\rangle)$ near the center. The second probe is scanned across the lower slope of the lower normal mode $(|0, g\rangle \rightarrow|1,+\rangle)$ where the crossed transition to the lower state in the second doublet $|1,+\rangle \rightarrow|2,-\rangle$ is expected at $\Delta_{2} \approx \sqrt{2} g+\Delta_{1}$.

The transmission is averaged for probe intervals of $0.1 \mathrm{~ms}$, qualified by the transmission of a single frequency in both neighboring cooling intervals to be below $4 \%$ of the empty cavity value. During the $0.5 \mathrm{~ms}$ cooling intervals a single probe resonant with the empty cavity at $0 \mathrm{MHz}$ and a power of $I_{\mathrm{p}}=0.3 \mathrm{pW}$ is used to compensate for the introduced heating by cavity cooling. During the probe intervals each probe beam has a power of $I_{\mathrm{p}}=0.1 \mathrm{pW}$. The power is switched in less than $10 \mu \mathrm{s}$.

In the spectrum a bump is noticeable at the position of the expected crossed transition. The two (one) Gaussian least square fits shows a reduced $\chi^{2}$ of $0.83(1.71$ ), respectively. The fitted position and width (FWHM) of the normal mode is $\Delta_{\mathrm{nm}} / 2 \pi=$ $-10.8 \mathrm{MHz}, \delta_{\mathrm{nm}} / 2 \pi=11.1 \pm 0.6 \mathrm{MHz}$, respectively. The shoulder is at $\Delta_{\mathrm{ct}} / 2 \pi=$ $-21.1 \mathrm{MHz}$ with a width of $\delta_{\text {ct }} / 2 \pi=3.3 \pm 0.7 \mathrm{MHz}$.

The position of the resonances can be analyzed by evaluating the ratio of the detunings with respect to the known empty cavity. This ratio can be expanded taking into account a small residual atom-cavity detuning $\Delta_{a c}$ :

\footnotetext{
(i.) Note that all the spectra in Fig. 8.4 indicate a peak structure on the lower tail of the upper normalmode peak, which is even more pronounced in the numerical simulation which do not take into account excitation into higher states. Therefore, this effect is most likely explained by a combination of the atomic motion and the qualification. Since the qualification removes the contribution of weakly coupled atoms it effects the spectrum the strongest in the vicinity of the empty cavity $(-2.5$ to $5 \mathrm{MHz})$.
} 


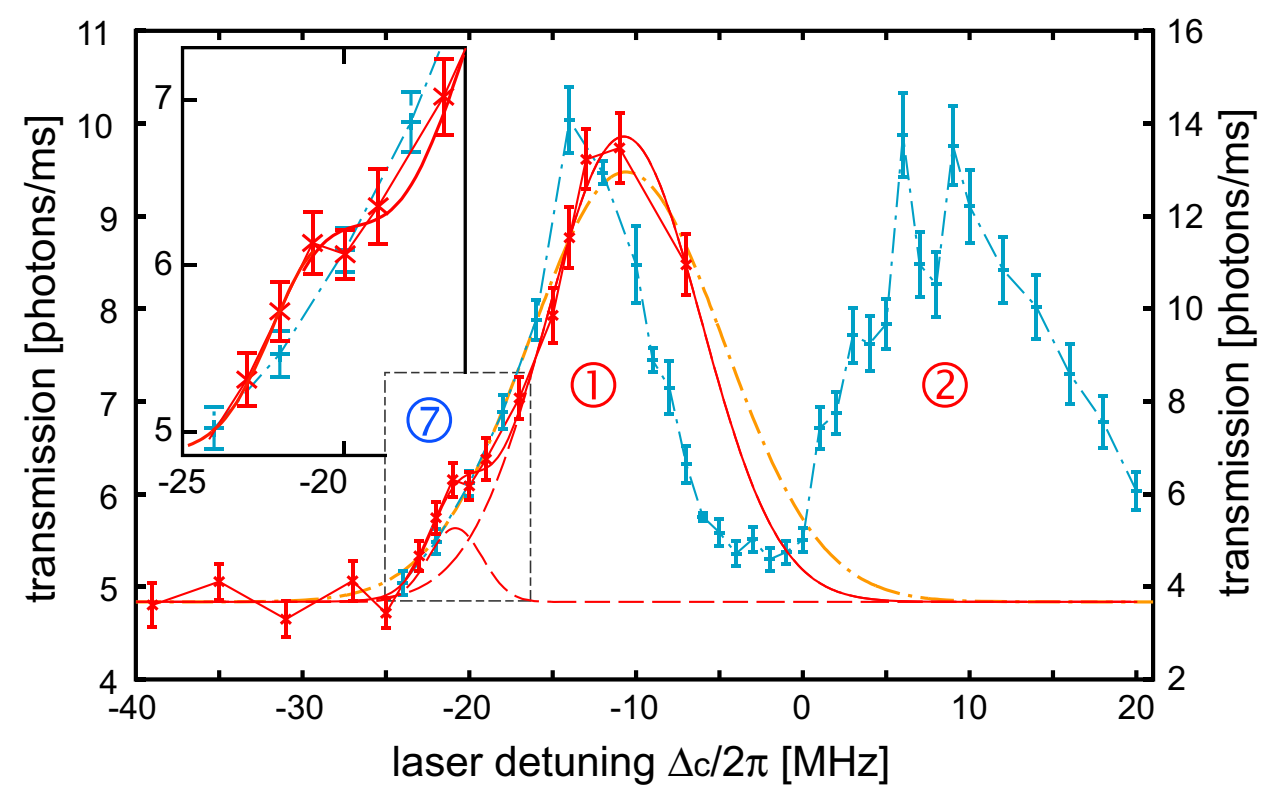

Figure 8.9: Two-photon transition in the red dipole trap. The bichromatic spectrum taken with one probe exciting the upper normal mode $(|1,+\rangle)$ at $11 \mathrm{MHz}$ and the second probe scanned across the expected crossed transition to the lower state of the second manifold $|2,-\rangle$. Fits of a single (orange dashed-dotted) and two Gaussians (red solid, separate as red dashed ) to quantify the observed structure are discussed in the text. The position of the shoulder at $\Delta_{2 \mathrm{ph}}=2 \pi \times-21.1 \mathrm{MHz}$ of the normal-mode peak at $\Delta_{\mathrm{nm}}=2 \pi \times-10.8 \mathrm{MHz}$ coincides with the expected resonance and is enlarged in the insert. The near-resonant normal-mode spectrum (blue dashed-dotted) for a trap depth of $P=310 \mathrm{nW}$ is shown for reference (right scale).

$$
\begin{aligned}
R_{\Delta} & =\frac{E_{2,-}-E_{1,+}}{E_{1,+}} \\
& \approx(1+\sqrt{2})\left(1-\frac{\Delta_{a c}}{2 g}\right)+\mathcal{O}\left(\frac{\Delta_{a c}}{g}\right)^{2} .
\end{aligned}
$$

Solving this equation with respect to the atom-cavity detuning, the calculated value $\Delta_{a c} / 2 \pi=2.3 \mathrm{MHz}$ is in good agreement with the value of about $3 \mathrm{MHz}$ derived from a fit to the normal-mode spectrum.

The ratio of the fitted width to the expected values correspond to inhomogeneous line broadening by factors of 2 and 2.5, respectively. The broadening of the spectrum is explained by the simultaneous variation of the coupling and the Stark shift for different positions of the atom in the mode. The dynamics of the atom is driven by the light forces and diffusion which show a strong dependency on the detuning in the probe interval. It remains to be explained, why the two-photon excitation is less susceptible to the broadening mechanism. Good agreement with the numerical simulation is found for the normal-mode splitting (see Sec. 8.2). Further insight into the influence of dynamical 
effects could be gained by numerical simulation including the motion of the atom as presented for the vacuum-Rabi splitting in Sec. 8.2. However, simulations are complicated by the fact that the low-excitation limit does not hold and moreover, by the time dependence introduced by using two probe fields.

In an additional measurement with a different detuning of the first frequency $\Delta_{1} / 2 \pi=$ $13 \mathrm{MHz}$ a structure shifted correspondingly to the opposite direction was observed. This excludes effects based on the frequency difference of the two probe beams like, e.g., Raman transitions between motional states of the trap. The weak pumping is a negligible perturbation such that the resonance structure is defined by the system. It was checked that a detailed measurement of the empty cavity transmission does not show any feature at the position of the shoulder. This excludes a signal from higher-order cavity modes.

\subsubsection{Two-photon spectrum in the blue trap}

To improve the conditions for measurements on the qualified strongly-coupled atomcavity system, the blue trap was developed and implemented (Chap. 7). Because the atom is stored close to zero intensity of the trapping fields, the contribution of the trap depth to the Stark shift is eliminated. This reduces the broadening of the spectra and allows to use very high confinement in the axial direction, which cannot be qualified. Since the bare atom detuning is preserved, the blue trap allows to access a larger parameter regime compatible with guiding and detecting the atom.

Figure 8.10 shows the measurement of the bichromatic two-photon step transitions for $\Delta_{a c}=-20 \mathrm{MHz}$. As discussed before, the detuning allows to better separate the transition from first to second doublet from the transition to the first doublet (see Fig. 8.1, dashed line). The first laser is fixed at resonance with the lower dressed state $|1,-\rangle$. This is the atom-like normal mode, therefore the system is only excited via the cavity, when the atom is strongly coupled. The second probe is scanned across the expected transitions from $|1,-\rangle$ to the second doublet. The cross-transition $|1,-\rangle \rightarrow|2,+\rangle$ is expected at $\approx 40 \mathrm{MHz}$. The data (not shown) does not show any structure neither in the transmission nor in the loss rate. This can be explained by the fact that this transition is nearly forbidden. The overlap is small because it corresponds to putting one extra excitation into the cavity while transferring the excitation from the atom to the mode. In contrast, the step-wise excitation $|1,-\rangle \rightarrow|2,-\rangle$ expected at $\approx-5 \mathrm{MHz}$ has a strong overlap with the pump, because it largely corresponds to an extra excitation of the mode. However, it is only possible when the strongly coupled system is excited by the first probe. The measured data shows an enhanced transmission at the position of the expected transition. The theory in the low-excitation limit does not include the excitations to the second doublet and is hence inadequate for the description of two-photon spectrum. However, it is well established to correctly reproduce the vacuum-Rabi splitting as shown in Fig. 7.5 for $\Delta_{a c} / 2 \pi=-35 \mathrm{MHz}$. The corresponding transmission for a well coupled atom-cavity system $\left(g=0.8 \times g_{0}\right)$ and $\Delta_{a c} / 2 \pi=-20 \mathrm{MHz}$ including the excitations to the first doublet only, is plotted for comparison. A coupling of $>0.8 \times g_{0}$ was achieved for the normal-mode splitting in the blue trap (section 7.4). The observed structure is well separated from the resonance to the first doublet. Moreover, its posi- 


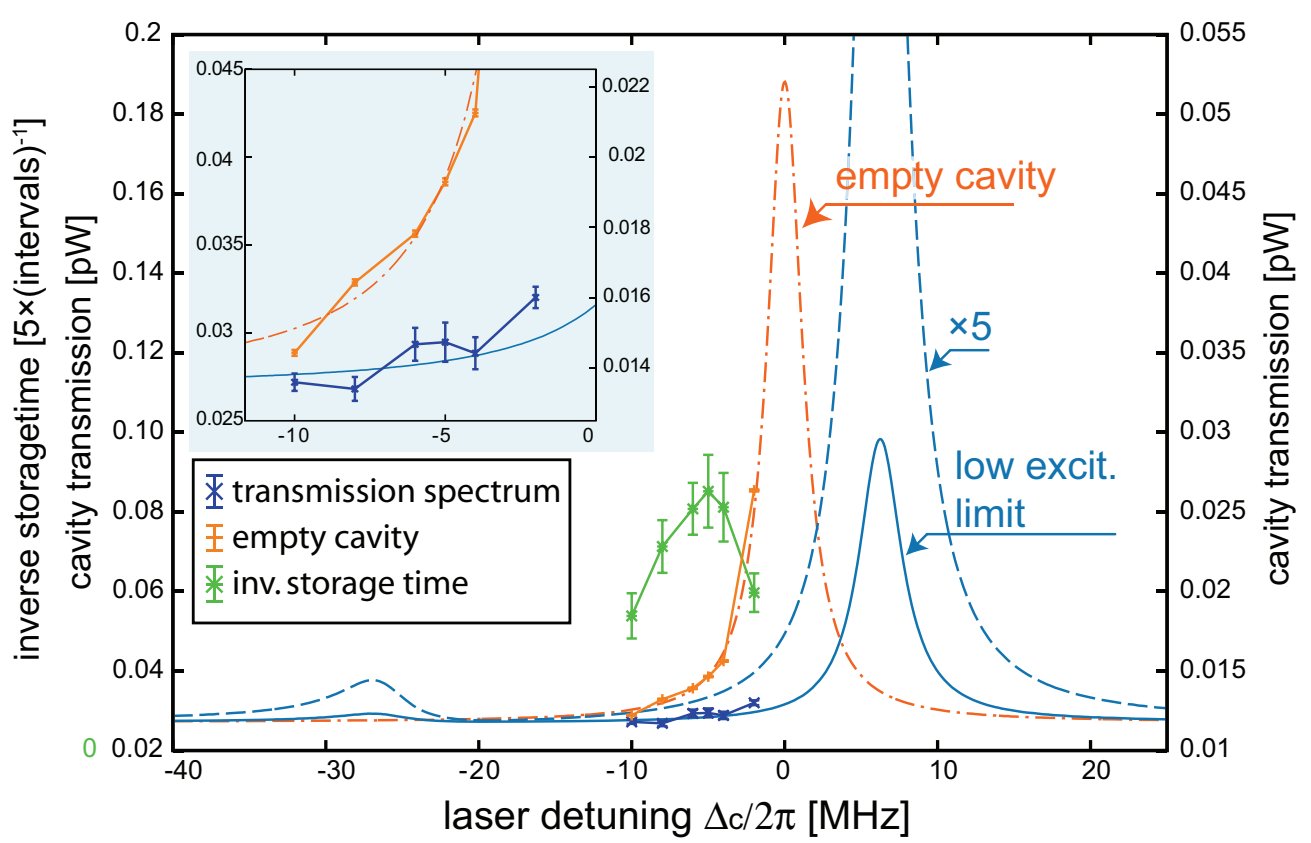

Figure 8.10: Two-photon transition in the blue dipole trap. The plot shows the transmission spectrum of the uncoupled (red) and coupled system (blue) on the left and right scale, respectively. The expected vacuum-Rabi spectrum (blue line) and the empty cavity (red line) are plotted for reference. One of the probes excites the atom-like normal mode. The other is scanned across the expected single-sided two-photon resonance, cf. Fig. 8.2. The transmission shows an increase at this position. This resonance is confirmed by a peak in the inverse storage time due to the induced heating on the resonance (green, left scale zero baseline).

tion is red detuned with respect to the bare cavity resonance. The transmission signal is confirmed by a pronounced peak in the excess loss rate representing a clear resonance.

To further confirm the signal, it is straightforward to repeat the experimental checks performed for the measurement in the red trap: 1. Measuring the one photon background without the fixed frequency probe to outline the normal mode (Fig. 7.5). 2. Using a different fixed frequency to observe a corresponding shift in the peak position. As discussed before, such measurements exclude effects that depend on the difference of the two probe frequencies.

The first signals of two-photon transitions of a strongly-coupled atom-cavity system with a single trapped atom demonstrate the potential for future experiments on the quantum nature of this fundamental combined quantum system. The developed bichromatic spectroscopy in the detuned system prepared in the blue trap is particularly promising for further investigations. 


\section{Chapter 9}

\section{Kaleidoscope}

The measurement of the position of the atom is based on its significant modification of both the amplitude and the phase of a weak light beam transmitted through the cavity. It has been predicted that the position of a slowly moving atom can be measured with an accuracy well below the standard diffraction limit, defined as half the wavelength of the employed light (100; 143). However, experimental requirements are challenging. The current status of the experiment allows to prepare a strongly-coupled atom cavitysystem with a slow atom passing the mode or stored in a dipole trap. The shot noise limited transmitted signal obtained from the single photon counters does not allow to observe the axial motion in real time. Assuming a given averaging in the axial direction the transmission is a measure for the mean coupling and therefore the atomic position in the transverse plane. Most of the experiments use the fundamental TEM $\mathrm{T}_{00}$ mode with a Gaussian profile. In this case the coupling is cylindrically symmetric. A onetime measurement of the transmission only allows to deduce the transverse distance corresponding to single value measured. Based on the assumption of nearly conserved angular momentum, the trajectory of the atom can be dynamically reconstructed for a subgroup of traces (101). This is confirmed by comparison with simulated trajectories. The atomic 'kaleidoscope' utilizes higher order modes to obtain more detailed position information at one time. Before going into the details of the atomic kaleidoscope the next section shortly introduces the higher-order modes.

\subsection{Higher order modes}

In a near-planar Fabry-Perot cavity the field is well described in paraxial approximation by a standing wave in direction of the cavity axis $(z)$ and Hermite-Gaussian mode patterns $\psi_{n, m}(\mathbf{r})$ in the transverse plane:

$$
\psi_{n, m}(\mathbf{r})=\left(w_{0}^{2} \pi / 2\right)^{-\frac{1}{2}} \cos (k z) e^{-\left(x^{2}+y^{2}\right) / w_{0}^{2}} H_{n, m}(x, y),
$$

where $k=\frac{2 \pi}{\lambda}$ is the wave vector and $\lambda$ the wavelength. The waist of the mode $w_{0}=\sqrt{\frac{\lambda}{\pi} z_{r}}$ is given by the Rayleigh length $z_{r}=\frac{l}{2} \sqrt{\frac{2 R}{l}-1}$ which depends on the radius of curvature 
of the mirrors in units of the cavity length $l$. The Hermite-Gaussian mode patterns are given by products of Hermite polynomials in $x$ and $y$

$$
\begin{aligned}
H_{n, m}(x, y) & =C_{n, m} H_{n}\left(\frac{\sqrt{2} x}{w_{0}}\right) H_{m}\left(\frac{\sqrt{2} y}{w_{0}}\right) \\
C_{n, m} & =\left(2^{n} 2^{m} m ! n !\right)^{-\frac{1}{2}}
\end{aligned}
$$

The normalization constant $C_{n, m}$ is chosen to fulfill

$$
\int_{-\infty}^{\infty} d x \int_{-\infty}^{\infty} d y H_{n, m}(x, y)=1
$$

The coupling constant $g(\mathbf{r})$ scales with the field at the atomic position $\mathbf{r}$. It is given by $g_{0} \psi_{n, m}(\mathbf{r}) / \psi_{0,0}(\mathbf{r})$, where $g_{0}$ is the maximum coupling in the antinode of the fundamental $\mathrm{TEM}_{00}$ mode. For ideal spherical mirrors the eigenfrequencies of the modes $\psi_{n, m}(\mathbf{r})$ are given by

$$
\begin{aligned}
\omega_{q, n, m} & =2 \pi \times\left[q+(n+m+1) \xi_{\text {trans }}\right] \frac{c}{2 l} \\
\xi_{\text {trans }} & :=\frac{\arccos \left(\sqrt{g_{i} g_{o}}\right)}{\pi},
\end{aligned}
$$

where $q$ is the longitudinal mode order and the $g$ parameters given by $\left(g_{x}=1-\bar{g}_{x}, \bar{g}_{x}:=\right.$ $\frac{l}{R_{x}}, x \in i, o$ with the radii of curvatures $R_{i}\left(R_{o}\right)$ for the input and output mirror, respectively. For a near-planar cavity $R \gg l$ the distance between higher order modes of consecutive order is $\bar{\omega}_{\text {trans }} \approx \sqrt{\frac{\bar{g}_{i}+\bar{g}_{o}}{\bar{g}_{i} \bar{g}_{o}}} / \pi \times \mathcal{F}$ in units of the cavity linewidth $\kappa$. For the cavity in the experiment this frequency distance is 0.01 times the free-spectral range or $4.9 \times 10^{3}$ times the linewidth. The higher mode orders are closely spaced to higher frequency next to the fundamental $\mathrm{TEM}_{00}$ modes, when compared to the FSR, but well separated by many linewidths.

\subsection{Idea of the kaleidoscope}

The theoretical proposal of the atomic kaleidoscope (103) is based on using a set of frequency-degenerated higher-order transverse modes in combination with a spatially resolved detection. The underlying effect is that an atom positioned off axis breaks the otherwise perfect rotational symmetry of the cavity. With the atom present, there will be a unique superposition of the higher-order modes called the effective mode which maximizes the coupling at the given position (104, 111). The effective coupling depends only on the radial distance, owing to the common point symmetry of a set of higherorder modes for a given order. Therefore, only information on the radial position can be obtained from the integrated transmission. For higher-order modes, the mapping of the coupling to radial position is not unique and the spatial mode pattern is needed to extract the correct radial position. The azimuthal position is encoded in the orientation of the mode. When the system is excited via the atom from the side, the pattern 
emitted from the cavity will be given by this effective mode. It will generally show a bright area in the vicinity of the atomic position. Note that for a given mode order $N$ all modes have a common point symmetry, which will be preserved in the resulting mode pattern. This ambiguity could be removed by the choice of a special cavity length to achieve degeneracy of two sets of modes with odd and even order. If instead the cavity is excited, the excitation of the modes additionally depends on the overlap with the pump mode. For a given pump mode there is a second special superposition, the uncoupled pumped mode, which is the projection of the pump mode onto the uncoupled subspace. The transmission will then be the interference pattern of the effective mode and the uncoupled pumped mode. The amplitudes are determined by the overlap with the given pump and correspond to the coupled and uncoupled case of the single mode theory. The observed effect therefore depends on the detunings chosen for detection. For resonant detection the effective mode will be tuned out of resonance. For a strongly coupled atom the transmission will therefore largely correspond to the uncoupled pumped mode defined by the atomic position, i.e. the intensity in the vicinity of the atomic position will be decreased. With decreasing coupling this effect will be reduced by an increasing contribution of the effective mode. For dispersive detection the empty cavity transmission will be low. The effective mode will be tuned in resonance with the laser and, hence, there will be an increasingly bright region in the vicinity of the atom for increasing coupling.

\subsection{Experimental cavity modes}

In the current experiment, the perfect rotation symmetry is broken and the modes of each transverse order will generally be nondegenerate. The broken symmetry can be explained, e.g. by a deviation of the mirror surfaces from an ideal sphere. They can be considered as ellipsoids with two slightly different radii of curvature in the main axis. In a high-finesse cavity even a small deviation leads to an appreciable splitting with respect to the linewidth. The $(N+1)$ modes of the given mode order $N$ are therefore given by superpositions of the Hermite-Gaussian basis with coefficients $\alpha_{i, n, m}$

$$
\phi_{i}(\mathbf{r})=\left(\sum_{n+m \equiv N} \alpha_{i, n, m}^{2}\right)^{-\frac{1}{2}} \sum_{n+m \equiv N} \alpha_{i, n, m} \psi_{n, m}(\mathbf{r}), i=[0 . . N+1] .
$$

Already the modes of order $N=1$ (Fig. 9.1) are separated by many linewidths $(25 \mathrm{MHz})$. The eigenmodes of the cavity closely resemble the $\mathrm{TEM}_{10}$ and $\mathrm{TEM}_{01}$ Hermite-Gaussian modes, apart from a small tilt by approximately $5^{\circ}$. This is consistent with breaking the cylindrical symmetry with respect to two main axis as predicted with ellipsoidal mirror surfaces. Note that the main axis of the mirrors will generally not be aligned and the orientation of the modes is a residual degree of freedom. The frequency splitting and orientation of these modes actually changed in a time interval of about two years. During that time the vacuum system was vented once, but the cavity remained otherwise untouched. Now, the splitting is much smaller $(6 \mathrm{MHz})$ and the orientation is 


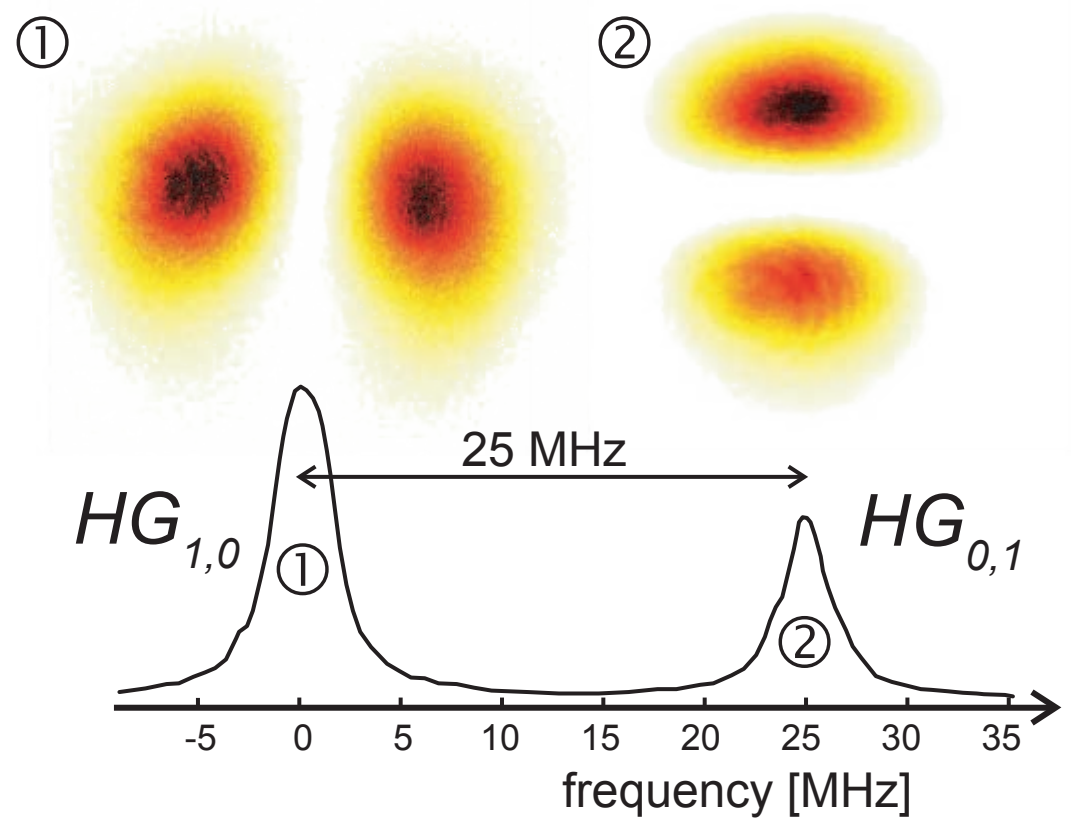

Figure 9.1: Experimental cavity modes of order $N=1$. The experimentally observed transmission patterns of the modes of order $N=1$ closely resemble the $\mathrm{TEM}_{10}$ and $\mathrm{TEM}_{01}$ Hermite functions. They are split by $25 \mathrm{MHz}$ which is much larger than the linewidth.

at $45^{\circ}$ (see Fig. 7.2). Fortunately, the significant overlap between the modes allows to excite superpositions as required for the blue trap, Chap. 7 .

In the mode order $N=2$, one of the modes (1) is well separated by $35 \mathrm{MHz}$ from the other two (Fig. 9.2). This mode is similar to a rotated TEM $_{20}$ mode. The other two are superpositions of the Hermite-Gaussian modes as can be seen by inspecting the center region. Especially mode (2) shows a bottleneck reminiscent of contribution of the $\mathrm{TEM}_{11}$ mode. As mentioned before, all modes are point symmetric and deviations thereof are attributed to abberations in the imaging system. The modes (2) and (3) overlap significantly. When one of the modes is probed resonantly, the other is still significantly excited off-resonantly. In between these modes superpositions can be excited. Because the relative phase of the excited eigenmodes depends on the geometry of the pump field, the resultant interference patterns observed in transmission do so as well.

The current spectrum of the modes of order $N=2$ is shown in Fig. 9.3. The splitting of the modes by approximately $12 \mathrm{MHz}$ is much more symmetric. Again all modes show three bright regions separated by two nodal lines and are turned by about $60^{\circ}$. Note that the orientation of the two higher frequency modes have changed. Since the modes significantly overlap, the spectrum of mode patterns shows different superpositions of eigenmodes. For the chosen pump geometry the mode patterns excited at the minima between the eigenmodes are close to $\mathrm{TEM}_{11}$ modes at $0^{\circ}$ and $45^{\circ}$ degrees, respectively. The $0^{\circ}$ mode has a nodal line that would be suited for guiding the atoms. The simul- 
A.

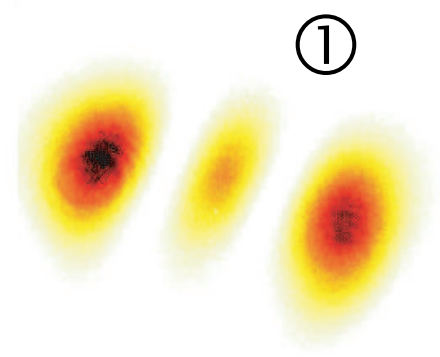

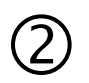

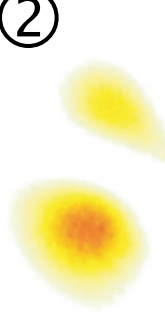

(3)

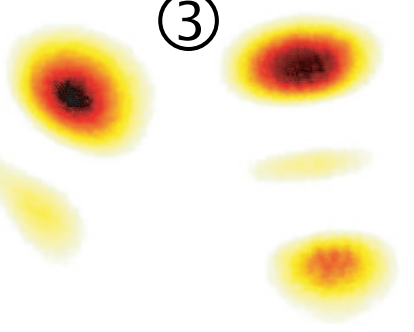

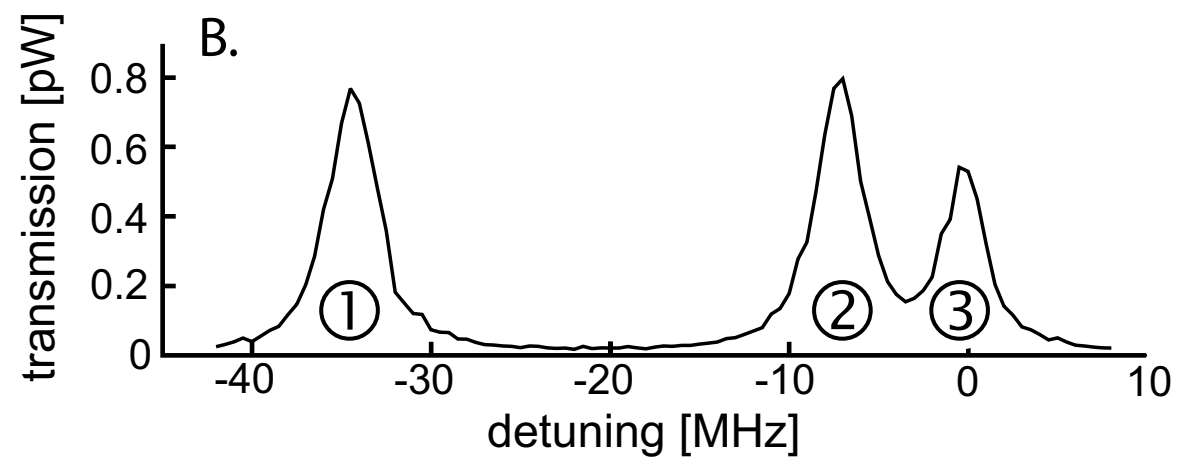

Figure 9.2: Experimental cavity modes of order $N=2$. The experimentally observed transmission patterns of the modes of order $N=2$ span a frequency range of $35 \mathrm{MHz}$. Each mode shows three bright regions separated by two nodal lines, turned by about $60^{\circ}$. The intensity pattern of the well separated mode (1) is close to a $\mathrm{TEM}_{20}$ mode while the significantly overlapping modes (2) \& (3) are superpositions.

taneous excitation of both modes would allow for three-dimensional confinement with a larger dark center region, when compared to the $\mathrm{TEM}_{01}+\mathrm{TEM}_{10}$ case. In principle a 'dark doughnut' consisting of a bright ring with a bright center is a possible superposition of the modes of order $N=2$. It would allow to trap atoms in dark toroids and the circular orbit could be observed by switching between $\mathrm{TEM}_{10}$ and $\mathrm{TEM}_{01}$ detection modes as shown for passing atoms in the next section.

\subsection{Transits through $\mathrm{TEM}_{10}+\mathrm{TEM}_{01}$}

A direct realization of the idea of the atomic kaleidoscope(Sec. 9.2) is not possible, because the cylindrical symmetry is already broken, as shown in the last section. The experimental cavity modes of a given order split by a few $\mathrm{MHz}$ and the geometry of the eigenmodes is fixed by the cavity. On the other hand, the frequency splitting can be utilized to deliberately address only one of the modes. In this case it is not necessary to spatially separate the transmission. The coupling of the $\mathrm{TEM}_{00}$ pump beam is adjusted to pump the modes of order $N=2$ (Fig. 9.3) with approximately the same strength. When the probe beam resonantly excites one of the modes, the other is detuned by $\approx 25 \mathrm{MHz}$. Its off-resonant excitation is reduced by a factor of $>300$ and can safely be 

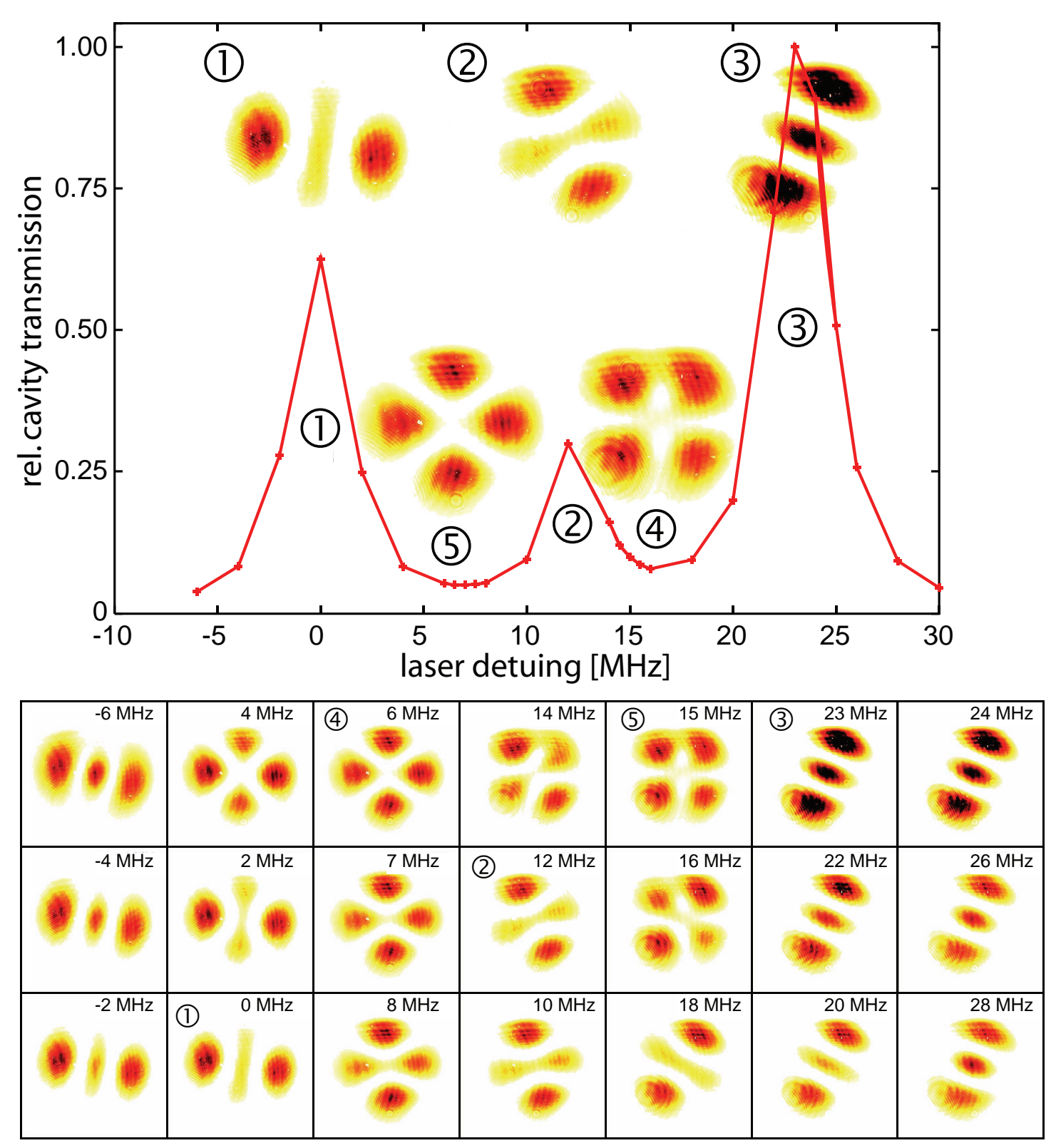

Figure 9.3: Current spectrum of the experimental cavity modes of order $N=2$. Currently, also the splitting of the modes of order $N=2$ is smaller $(25 \mathrm{MHz})$ than in Fig. 9.2 . The lowest frequency mode is still approximately oriented in the same direction but shows some deviation from the $\mathrm{TEM}_{20}$ mode. Now the highest frequency mode is close to a $\mathrm{TEM}_{20}$ mode, while the center mode again shows a contribution of the $\mathrm{TEM}_{11}$ mode. The continuous change in the spectrum of the mode patterns shows two TEM 11 modes, at $0^{\circ}$ and $45^{\circ}$, excited at the minima between the eigenmodes. 
neglected. Traces for resonant detection of single atom transits are shown in Fig. 9.4 , For resonant detection the transmission is the smaller the larger the coupling of the atom to the mode is. The transits through the $\mathrm{TEM}_{10}$ show a single dip while for the $\mathrm{TEM}_{01}$, double-dip structures are observed. These correspond to the two maxima in the coupling when the atoms pass the mode in vertical direction. For a detailed analysis the analytic theory (Sec. 2.4) is used to find an expression for the transmission signal averaging over the axial direction. Even though the entrance velocity in axial direction is geometrically selected to below one recoil $\left(6 \frac{\mathrm{mm}}{\mathrm{s}}\right)$, simulations show that due to momentum diffusion the axial momentum will be largely broadened(i.) . Hence, averaging the position dependent transmission (2.46) given by

$$
\frac{T(\mathbf{r})}{T_{0}}=\frac{\kappa^{2}\left|\tilde{\Delta}_{a}\right|^{2}}{|\mathcal{A}|^{2}}
$$

along the standing wave direction is well justified. The transverse velocity $\left(v_{\mathrm{y}}\right)$ is assumed to be constant neglecting the small effect due to the light forces of the cavity probe field. The straight trajectory of an atom is therefore given by the velocity $v_{\mathrm{y}}$ and the minimum radial distance $x_{0}$. The single atom transits through $\mathrm{TEM}_{10}$ and $\mathrm{TEM}_{01}$ modes are discussed in the following.

\subsubsection{Transits through TEM $_{10}$}

A transit through the $\mathrm{TEM}_{10}$ mode shows a single dip (Fig. 9.4 A.) Both $x_{0}$ and $v_{\mathrm{y}}$ enter into the width of the dip. The transverse velocity $\left(v_{\mathrm{y}}\right)$ is therefore fixed by the arrival time of the atom in the cavity mode by the ballistic trajectory to within the knowledge of the position of atomic cloud in the magneto-optic trap (MOT). For the sample trajectory at $t=147.13 \mathrm{~ms}$, i.e. $v_{\mathrm{y}}=1.07 \frac{\mathrm{m}}{\mathrm{s}}$, the closest distance to the cavity axis is fitted to $x_{0}=0.48(16) w_{0}$. Note that for a given signal a total of four equally possible trajectories at $x_{0}= \pm 0.48 w_{0}$ and $x_{0}= \pm 1.64 w_{0}$ exist.

\subsubsection{Transits through TEM $_{01}$}

In contrast, for the double-dip structure of transits through the $\mathrm{TEM}_{01}$ (Fig. 9.4, B.) the fixed vertical distance of $\sqrt{2} w_{0}$ between the two coupling maxima defines a natural ruler and the arrival time is well defined by the center peak. Hence, it is possible to independently determine the vertical velocity $v_{\mathrm{y}}=1.1(1) \frac{\mathrm{m}}{\mathrm{s}}$ and the two times degenerate minimum distance $x_{0}= \pm 1.0(1) w_{0}$ at $t=147.06 \mathrm{~ms}$. The velocity $v_{\mathrm{y}}$ agrees with the one expected from the ballistic trajectory $v_{\text {bal }}=1.07 \frac{\mathrm{m}}{\mathrm{s}}$.

\subsubsection{Simultaneous transits through TEM $_{01} \& \mathrm{TEM}_{10}$}

The transits discussed so far are taken for both atom and probe beam resonant with the chosen eigenmode. The transmission is a direct measure of the coupling $g$ to the

\footnotetext{
(i.) Note that in the experiment discussed here no dipole trap was installed
} 
A.
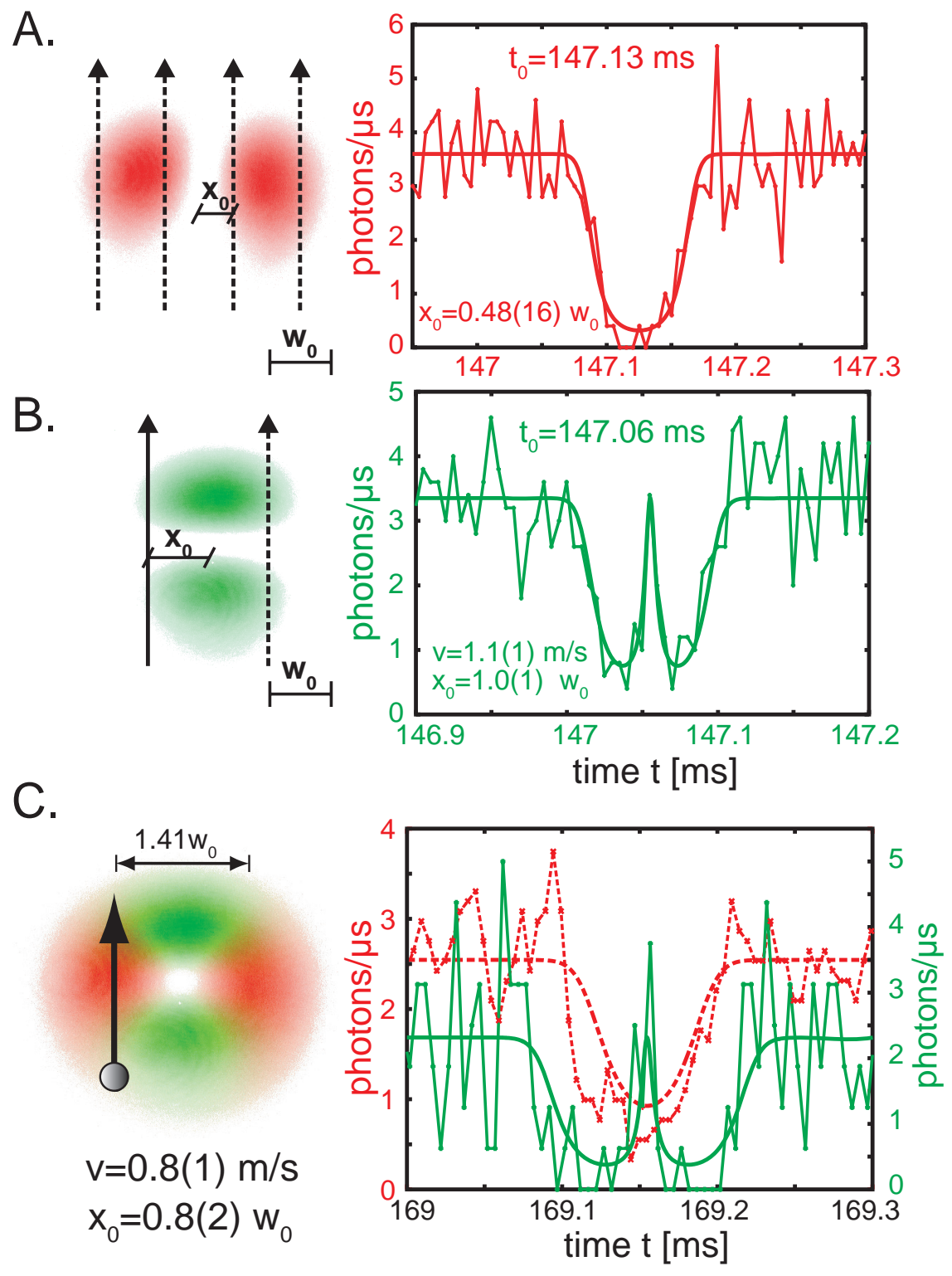

Figure 9.4: Transits through modes of order $N=1$ : A single atom passing through a $\mathrm{TEM}_{10}$ mode (A.) and a TEM 10 mode (B.) is observed by monitoring the transmission of the cavity. The weak probe laser is resonant with both the atom $\left(\Delta_{\mathrm{a}}=0\right)$ and the respective cavity mode $\left(\Delta_{c}=0\right)$. From a fit based on equation (2.45) (smooth curve), the arrival time of the atom at the center of the cavity, $t_{0}$, the minimum distance to the cavity axis, $x_{0}$, and in case of the $\mathrm{TEM}_{01}$ mode also the vertical velocity, $v_{\mathrm{y}}$, can be determined. C. Transmission during the transit of a single atom through alternatively excited $\mathrm{TEM}_{10}$ and $\mathrm{TEM}_{01}$ modes. The detunings are $\left(\Delta_{\mathrm{a}}, \Delta_{\mathrm{c}}\right) / 2 \pi \times=(-25,0) \mathrm{MHz}$ for the TEM 10 mode, and $\left(\Delta_{\mathrm{a}}, \Delta_{\mathrm{c}}\right) 2 \pi \times=(0,0) \mathrm{MHz}$ for the $\mathrm{TEM}_{01}$ mode. The left (right) vertical axis denotes the photon flux at the TEM $\mathrm{TE}_{10}\left(\mathrm{TEM}_{01}\right)$ mode frequency. Fitting a constant-velocity vertical trajectory to the experimental data, the velocity, $v=0.8(1) \frac{\mathrm{m}}{\mathrm{s}}$, and the minimum distance to the cavity axis, $x_{0}=0.8(2) w_{0}$, the arrival time of $t_{0}=169.15 \mathrm{~ms}$, is obtained.The signal-to-noise ratio of the observed events is given by the shot noise of the small number of photons detected. The rates are limited by the condition of weak probing. 
given higher-order mode. For sufficiently high signal-to-noise, the atomic position can be inferred from the contours of equal coupling. Multiple quasi simultaneous recordings of the coupling to different modes allow to pin the position of the atom to one of the intersection points of these contours. Using both $\mathrm{TEM}_{01}$ and $\mathrm{TEM}_{10}$ allows to determine the atomic position from one time measurements to one of the eight crossings of the two pairs of rings.

Figure 9.4 (C.) shows the alternating measurement of the couplings to the $\mathrm{TEM}_{01}$ and TEM $_{10}$ modes. The atom is resonant with the $\mathrm{TEM}_{01}$ mode while the probe laser is rapidly switched between the two modes with a frequency of $200 \mathrm{kHz}$. The intervals of $2.5 \mu \mathrm{s}$ are much shorter than the timescale of the transit of a few $100 \mu \mathrm{s}$. Because the light field in the cavity equilibrates in $300 \mathrm{~ns}$ after switching, photons during these times are discarded. Unfortunately, the signal-to-noise of the experimental signal, limited by fundamental shot noise, is not sufficient to derive an atomic position from a single pair of couplings. However, for the displayed transit event, enough information is available to estimate the velocity and the horizontal position of the trajectory. The fit to the $\mathrm{TEM}_{01}$ determines the velocity, $v=0.8(1) \frac{\mathrm{m}}{\mathrm{s}}$, the minimal distance $x_{0}=0.8(2) w_{0}$, and the arrival time of $t=169.15 \mathrm{~ms}$. The velocity agrees with the expected $v_{\text {bal }}=0.72 \frac{\mathrm{m}}{\mathrm{s}}$. The deduced transmission signal for the $\mathrm{TEM}_{01}$ mode calculated without any further free parameter matches the experimental data reasonably well. The effect that the experimentally observed transmission of the $\mathrm{TEM}_{10}$ mode tends to run below the theoretical expectation could be explained by an increased atom-cavity coupling. For the $\mathrm{TEM}_{10}$ mode the probe resonant with the cavity is red detuned from the atomic transition. Hence, the dipole force attracts the atom towards the antinodes of the cavity mode. Note that increasing the mode order $N$ generally increases the effective coupling extending the transverse detection region by $\sqrt{N}$. At the same time the number of contours of equal coupling for each higher-order mode increases and renders the reconstruction of the common intersection more complicated. Moreover, effective measurement time per mode in the alternating switching method decreases and the shot noise issue is enhanced. In principle, this could be counteracted by skipping a subset of modes which will not contribute to determine the current position based on the expectation from the last measurement. A more powerful solution would be simultaneous measurements by either geometrical selection or frequency selection by heterodyne detection.

\subsection{Atom in blue-detuned laser fields}

The measurements presented in this chapter so far were done before the implementation of an additional far-detuned intracavity dipole trap. The blue guiding fields allow to confine the atoms to regions of strong coupling. Especially, the axial confinement to the antinodal plane reduces position averaging. The enhanced coupling improves the detection. An interesting application would be to trace the rotation of an atom in the cylindrically symmetric blue trap, i.e. the 'dark doughnut', by detecting the coupling to the $\mathrm{TEM}_{10}$ and $\mathrm{TEM}_{01}$ modes. In a further step the azimuthal position information could then be used to apply feedback to the $\mathrm{TEM}_{10}+\mathrm{TEM}_{01}$ modes for transverse 


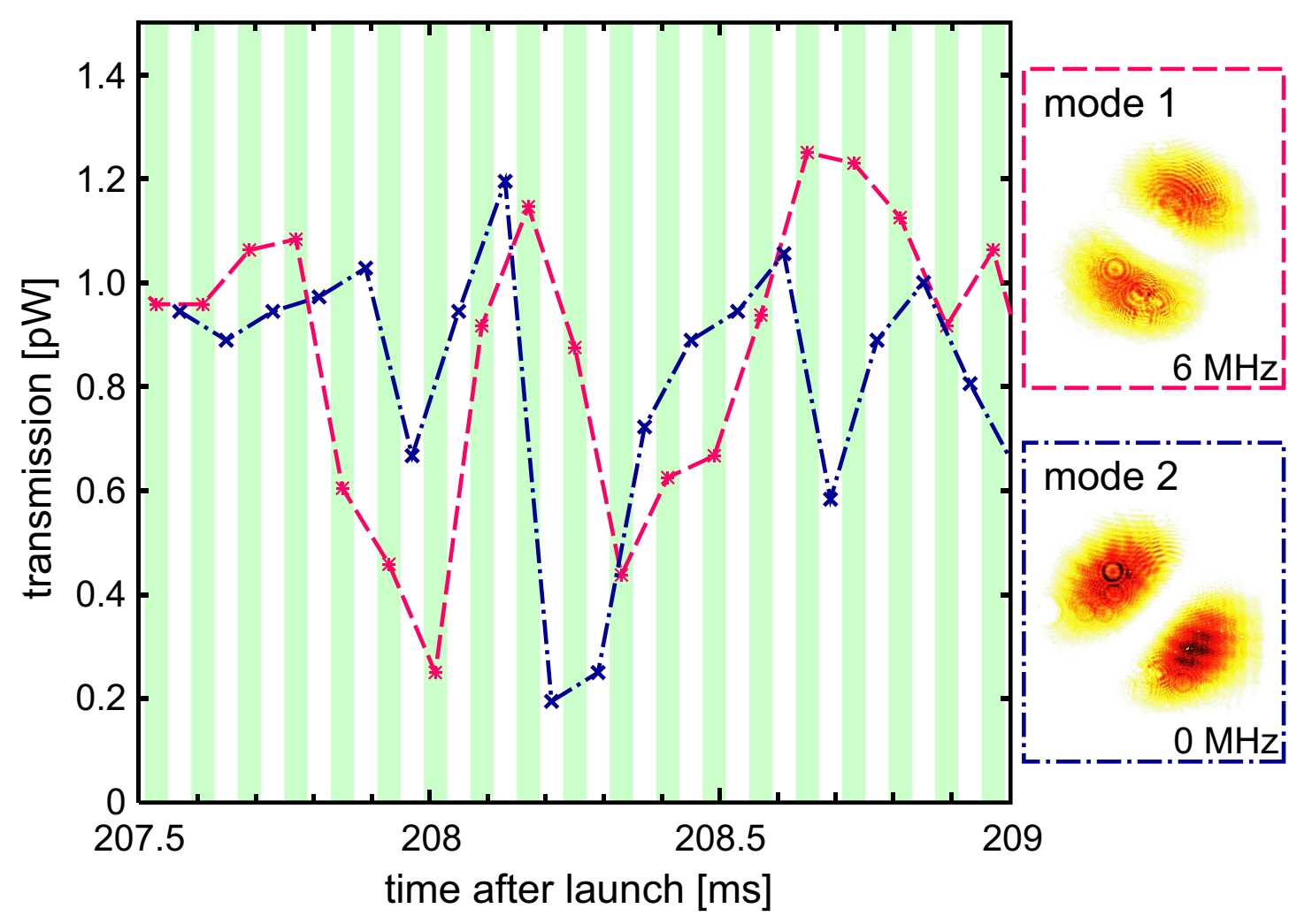

Figure 9.5: Guided transit through modes of order one. A single atom guided by blue dipole fields is passing through the $\mathrm{TEM}_{01}$ and $\mathrm{TEM}_{10}$ modes at $\pm 45^{\circ}$. The probe beam alternates between resonant excitation of the two modes in the different intervals indicated by the shading.

confinement to achieve azimuthal parametric cooling. The possibility to independently switch only the slow transverse confinement is a major advantage of the blue trap. The typical timescale of the addressed rotational motion correspond to the transit times of a few $100 \mu$ s and is hence well within the possibilities of feedback electronics.

Fig. 9.5 shows a first signal of the transit of a guided atom through the $\mathrm{TEM}_{10}$ and $\mathrm{TEM}_{01}$ modes with alternating resonant detection. Since in the meanwhile these modes are oriented in $\pm 45^{\circ}$ the interpretation of the signals for a simple transit is more complicated. In the sample trace the atom couples twice to both modes with reversed coupling strength as expected for a transit of an atom. 


\section{Chapter 10}

\section{Outlook}

The blue-detuned intracavity dipole trap presented in this thesis realizes a strongly coupled atom-cavity system, while largely preserving the free-space properties of the atom. The atom is effectively captured without increase in energy. It is stored close to the dark trap center where the Stark shift vanishes, well isolated from the environment by the surrounding potential barrier. Because the trap height does not contribute to the Stark shift, the confinement is only technically limited. The axial trap height realized is about an order of magnitude larger than in the red trap. The accessible parameter regime is not limited by the shift in atomic detuning. The preserved large detuning realize the regime of dispersive detection, where the presence of an atom can be detected while it only scatters about one photon. It is a major step towards a controlled preparation with a minimum impact on the system under study. Its potential for fundamental studies in cavity QED is demonstrated by the first observation of a two-photon resonance.

Combining the blue trap with three-dimensional cavity cooling would fully establish the possibility of continuous measurements on a single open quantum system. Because three-dimensional cavity cooling counteracts radial heating it can enhance the storage times by several orders of magnitude (137) and achieves significant population of the motional ground state. As a proof of principle, atoms are successfully stored in the blue trap for the parameter regime compatible with three-dimensional cooling. Unfortunately, limited optical access from the side in the current setup prohibits the immediate implementation. An alternative method to achieve (axial) motional ground state cooling in a state-insensitive trap (95) is Raman side-band cooling (130). Ground state cooling enters the regime where in addition to the field and the atomic dipole also the atomic motion has to be quantized. It has been realized for ions (144; 145) and enabled quantum information processing (146). In cavity QED qualitatively new phenomena are predicted based on the quantum correlations between the internal and the motional degrees of freedom due to their interaction (147; 148), e.g. the transfer of a quantum state between motion and light (149) and the realization of a Einstein-Podolsky-Rosen state of distantly separated trapped atoms (150). Measuring one of the components will give rise to a backaction on the dynamics of the other $(151 ; 152$; 143 ; 153$)$ which can lead to nonclassical effects, e.g. the localization of the atom (153) and motional Schrödiger 
cat states induced by the mode symmetry and dissipation (154).

The atomic motion of an atom in the cavity can be actively controlled by feedback based on the radial position information obtained from the cavity transmission (102). The blue trap now allows independent feedback on the radial potential only, while the axial confinement is preserved. Moreover, azimuthal feedback could be implemented based on the two-dimensional position information provided by the atomic kaleidoscope (103; 104). Different trap topologies are possible by a combination of appropriate higherorder modes, for example a two-dimensional closed dark ring of mesoscopic size. The rotation of a single trapped atom could then be observed using the $\mathrm{TEM}_{10}+\mathrm{TEM}_{01}$ modes (105). In combination with the ability to individually address the confinement in different directions in the blue trap, azimuthal feedback could be applied. This configuration, resembling a Sagnac interferometer, realizes an atomic gallery leading to very interesting dynamics of a matter wave (155).

A different system that aims at the study of the dynamics of matter waves in controlled potentials are atom chips. The small scale structures provide strong magnetic field gradients and hence strong confinement (156, 157, 158, 159). The chips can be patterned to implement optics for matter waves, e.g. beam splitters (160; 161). They are well suited for single atoms as well as convenient sources of cold atomic clouds and Bose-Einstein condensates (162, 163). Driving applications are the realization of an integrated mesoscopic atom interferometer (164) and the manipulation of single atoms for quantum computing (165). Single atom detection can be provided by integrating cavities (131; 132; 133; 134). A first application as a single atom detector has been the study of the atom statistics in an atom laser (135). In these settings the blue funnels, as realized in this thesis, could efficiently guide atoms to regions of large atom-cavity coupling, thereby enhancing the detection efficiency.

Toroidal microcavities (166; 167) are a different approach to chip integration. Lithographic chip technology and selective reflow is used to produce these ultrahigh-Q small volume micro-resonators (168) based on total internal reflection. Here, the coupling to the evanescent field mode of the cavity can be controlled via the tapered fibre (169). Several of these resonators can be readily integrated which allows for a certain scalability in the number of cavities. Single-atom detection (170) and strong coupling have been observed (171), but trapping atoms in the vicinity of the surface is still a challenge. An additional aspect are the mechanical modes of the toroidal structure which can be cavity cooled by radiation pressure coupling (172). To achieve cavity cooling to the ground-state the sidebands of the mechanical motion must be addressed individually by the cavity resonance. Hence the oscillation frequency must be larger than the cavity linewidth (136). This regime is also attainable for the axial confinement in the blue trap.

Improved control and deterministic preparation of atom-cavity systems with a few atoms in Fabry-Perot cavities are directed towards deterministic single photon sources (62; 63) for quantum computing with linear optics (67, 146). Atoms can be deterministically transported $(39,119,173)$ and localized in the mode of the high-finesse cavity (118). A desired number of atoms can be prepared in a dipole trap (174) or by monitoring the 
cavity transmission (175). Recent advances in the realization of a quantum node include the mapping of the internal state of an atom onto a photon (176) and reversible mapping between atom and photon (65). Triggered single photon sources can now operate for tens of seconds based on single trapped ions (177) and neutral atoms (66). They are well suited as sources for quantum cryptography (178) and to implement a matter-light interface for distributed entanglement in quantum networks (68). Using a blue-detuned intracavity dipole trap to store atoms in the cavity mode would reduce the Stark shift variations, allow for better axial localization and greatly reduce scattering of trap light, which leads to uncontrolled background photons and will eventually limit the storage time.

Quantum effects and measurement-induced dynamics of the atom-cavity system are generally washed out by variations in the coupling and hence only become feasible with improved localization. Nonlinear quantum optics for a two-state atom coupled to a cavity mode with only two photons gives rise to interesting quantum effects (179): The photon blockade observed in the magic trap (95) is based on the induced photon-photon interaction (81). It shows a sub-Poissonian anti-bunched photon stream in cavity transmission. A single photon source can hence be realized by pulsed excitation (63). The effective photon-photon interaction is the basis for a number of proposals for quantum information processing with photons, e.g. to realize quantum gates and entanglement. Quantum dynamics conditioned on a detection has been observed in atomic beam experiments, e.g. conditional phase shifts (180), relaxation of the intra cavity field (181) and nonclassical correlations in the transmitted field (182). However, the detailed analysis and understanding of such beam experiments is involved due to the presence of weakly coupled atoms (183). The blue funnels are an experimental implementation of the mask invoked in the proposal for photon correlation spectroscopy (77) to reduce the effect of weekly coupled atoms. Blue intracavity light fields are well suited achieve strong confinement to regions of strong coupling while keeping the modification to the atom-cavity system at a minimum. 



\section{Appendix A}

\section{Rubidium energy levels}

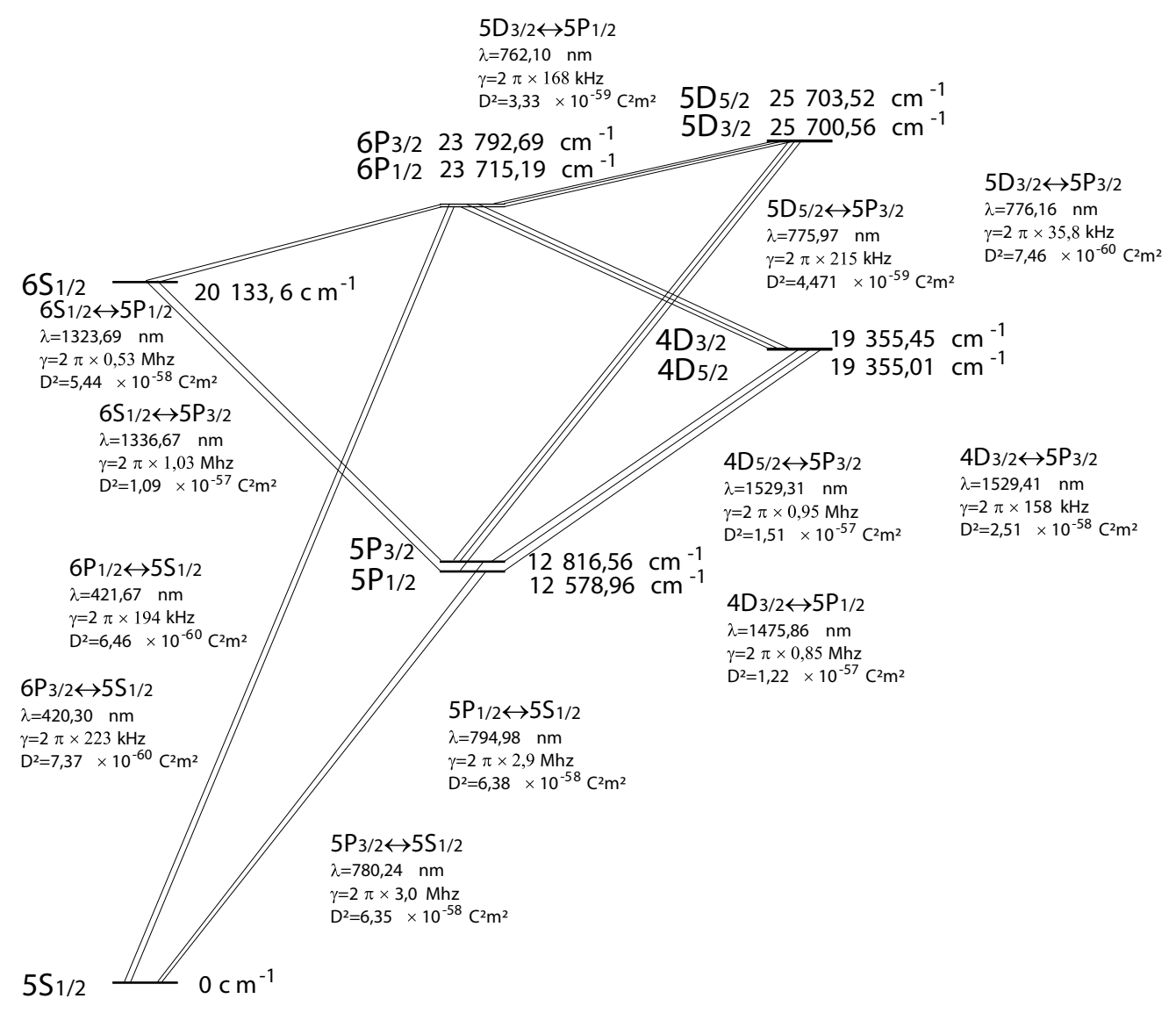

Figure A.1: Level scheme of Rubidium: Level scheme and transition strengths of Rubidium. The transition rate is $\gamma=\frac{1}{8 \pi \epsilon_{0}} \frac{4(2 \pi)^{3}}{3 h \lambda^{3}} D^{2}$ 



\section{Appendix B}

\section{Parameters in the numerical Simulations}

\begin{tabular}{|c|c|c|}
\hline Symbol & Parametername & Unit \\
\hline \hline$t_{\text {trig }}$ & Triggervalue & $\begin{array}{c}{[T] \text { where } T \text { is the transmission }} \\
\text { of the bare (empty resonant) cavity }\end{array}$ \\
\hline$P_{\mathrm{nr}}$ & $\begin{array}{c}\text { Power in the near-resonant probe mode } \\
\text { for detecting the atom }\end{array}$ & {$\left[w_{0}\right]$} \\
\hline$x_{0}$ & $\begin{array}{c}\text { interval on } x \text {-axis in which } \\
\text { the atom is randomly positioned }\end{array}$ & {$\left[w_{0}\right]$} \\
\hline$y_{0}$ & interval on $y$-axis & {$[\lambda]$} \\
\hline$z_{0}$ & interval on $z$-axis & {$[$ photons $]$} \\
\hline$\left(P_{\mathrm{nr}}, P_{\mathrm{nrs}}\right)$ & $\begin{array}{c}\text { Power in the guiding field of the red } \\
\text { dipole trap (before, after }) \text { switching }\end{array}$ & {$[\mathrm{photons}]$} \\
\hline$\left(P_{\mathrm{b} 0}, P_{\mathrm{b} 0 \mathrm{~s}}\right)$ & Power in the TEM & monode \\
\hline$\left(P_{\left.\mathrm{b} 1, P_{\mathrm{b} 1 \mathrm{~s}}\right)}\right)$ & Power in the TEM 10 and TEM 01 modes & {$[2 \pi \mathrm{MHz}]$} \\
\hline$\Delta_{\mathrm{a}}$ & bare atom detuning & \\
\hline$\Delta_{\mathrm{c}}$ & bare cavity detuning & \\
\hline
\end{tabular}

Table B.1: Parameters in the numerical Simulations. 



\section{Appendix C}

\section{Polarization of the high-finesse cavity modes}

Generally the measurements on the $\mathrm{TEM}_{00}$ mode did not show any significant birefringence. Here, the polarization of the cavity on the $\mathrm{TEM}_{10}+\mathrm{TEM}_{01}$ modes is analyzed base on cavity transmission spectrum for different settings of a $\frac{\lambda}{4}$ plate. The transmission $T$ detected behind a linear polarizer dependents oscillates with different multiples of the rotation angle $\alpha$ of the $\frac{\lambda}{4}$ plate:

$$
T=\frac{1}{2}\left(S_{0}+S_{1} \cos (2 \alpha)^{2}+S_{2} * \sin (2 \alpha) \cos (2 \alpha)+S_{3} \sin [2 \alpha) .\right.
$$

The Stokes parameters $S_{i}, i \in 0,3$ can directly be obtained from the coefficients of a Fourier analysis. They fully describe the polarization state of the photon field. The different characteristic parameters can be expressed in the Stokes parameters, for example the degree of (circular/linear) polarization $(\mathrm{DO}[\mathrm{C} / \mathrm{L}] \mathrm{P})$ and the parameters describing the polarization ellipse:

$$
\begin{aligned}
\text { DOP } & =\frac{\sqrt{\left(S_{1}^{2}+S_{2}^{2}+S_{3}^{2}\right)}}{S_{0}} \\
\text { DOLP } & \left.=\frac{\sqrt{\left(S_{1}^{2}+S_{2}^{2}\right.}}{S_{0}}\right) \\
\text { DOCP } & =\frac{S_{3}}{S_{0}} \\
\Theta & =\frac{1}{2} * \arctan \left(\frac{S_{2}}{S_{1}}\right) \\
r & =\frac{S_{0}}{S_{3}} *\left(1-\sqrt{\left.\left(1-\left(\frac{S_{3}}{S_{0}}\right)^{2}\right)\right) .}\right.
\end{aligned}
$$

The average transmission spectrum of all different settings of the $\frac{\lambda}{4}$ plate is well fitted by three Lorentzian showing a splitting on one of the modes of order 1 (see Fig. C.1). 


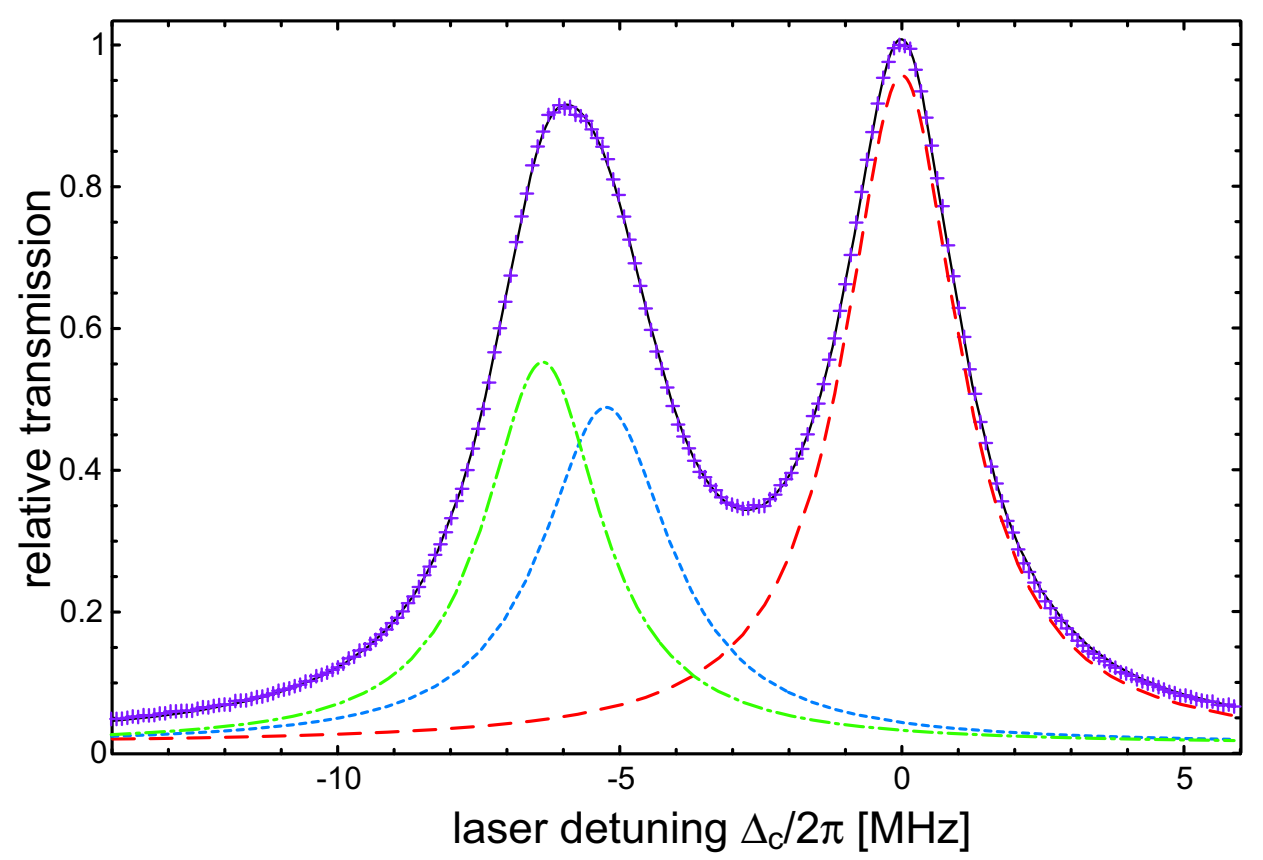

Figure C.1: Transmission spectrum for polarization analysis. The average transmission observed for different angles of the $\frac{\lambda}{4}$ plate in the polarization analysis is well fitted by three resonances

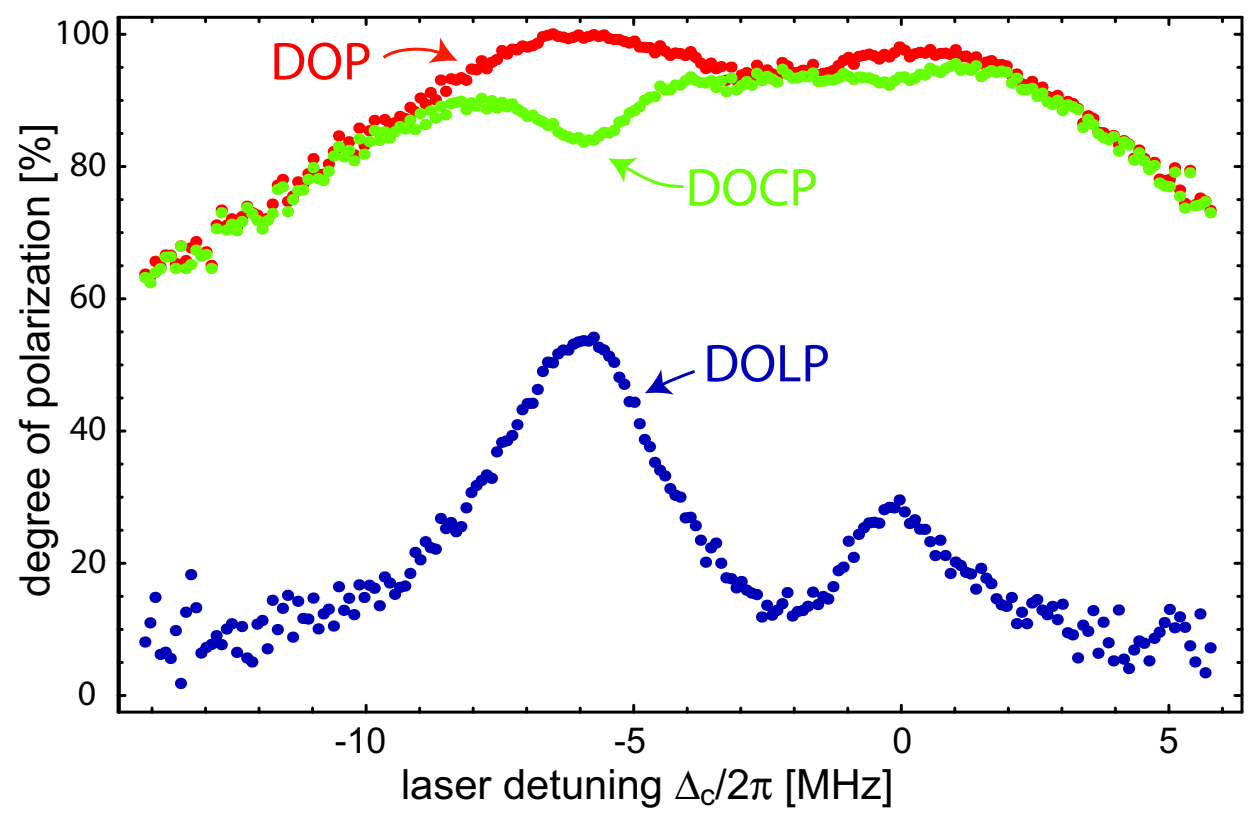

Figure C.2: Degree of polarization in transmission for a circularly polarized pump. While the total degree of polarization (DOP) is largely preserved, a reduced circular (DOCP) and significant linear contribution (DOLP) is observed on the resonances. The frequency dependence of the polarization is particularly strong on the peak that is fitted by two Lorenzians (cf. Fig. C.1) 
Figure C.2 shows the total degree of polarization as well as the circular and linear contributions. The fact, that the degree of polarization decreases to the borders is an artefact of the reduced signal. As expected, the total degree of polarization is largely preserved. However, the intended circular polarization is altered on the resonance and a significant linear contribution is observed. A stronger effect is observed for the resonance which is fitted by a two peak structure in Fig. C.1. Here, the shift between the polarization modes, defined by the cavity, limit the possibility to establish a precise polarization of the intracavity light by a given polarization of the pump. 



\section{Bibliography}

[1] D. F. Walls and G. J. Milburn. Effect of dissipation on quantum coherence. Phys. Rev. A 31, 2403 (1985).

[2] J. A. Wheeler and W. H. Zurek. Quantum Theory and Measurement (Princeton University Press, 1983).

[3] G. J. Milburn. Classical and quantum conditional statistical dynamics. Quant. Semiclass. Opt. 8, 269 (1996).

[4] E. Joos, H. D. Zeh, C. Kiefer, D. Giulini, I. Stamatescu, and J. Kupsch. Decoherence and the Appearance of a Classical World in Quantum Theory (Springer, Berlin, 2003).

[5] W. H. Zurek. Decoherence and the transition from quantum to classical - revisited. arXiv: quant-ph/0306072 (2003).

[6] H. J. Carmichael. An Open Systems Approach to Quantum Mechanics (Springer, 1993).

[7] A. Barchielli. Measurement theory and stochastic differential equations in quantum mechanics. Phys. Rev. A 34, 1642 (1986).

[8] L. Diosi. Continuous quantum measurement and ito formalism. Phys. Lett. A 8-9, 419 (1988).

[9] V. P. Belavkin. A continuous counting observation and posterior quantum dynamics. J. Phys. A 22, L1109 (1989).

[10] V. Belavkin. Theory of the control of observable quantum-systems. Automat. Rem. Contr. 44, 178 (1983).

[11] H. M. Wiseman and G. J. Milburn. Quantum theory of optical feedback via homodyne detection. Phys. Rev. Lett. 70, 548 (1993).

[12] A. C. Doherty, S. Habib, K. Jacobs, H. Mabuchi, and S. M. Tan. Quantum feedback control and classical control theory. Phys. Rev. A 62, 012105 (2000). 
[13] H. M. Wiseman and G. J. Milburn. Squeezing via feedback. Phys. Rev. A 49, 1350 (1994).

[14] J. Geremia, J. K. Stockton, and H. Mabuchi. Real-Time Quantum Feedback Control of Atomic Spin-Squeezing. Science 304, 270 (2004).

[15] H. M. Wiseman. Adaptive Phase Measurements of Optical Modes: Going Beyond the Marginal Q Distribution. Phys. Rev. Lett. 75, 4587 (1995).

[16] M. A. Armen, J. K. Au, J. K. Stockton, A. C. Doherty, and H. Mabuchi. Adaptive Homodyne Measurement of Optical Phase. Phys. Rev. Lett. 89, 133602 (2002).

[17] R. L. Cook, P. J. Martin, and J. M. Geremia. Optical coherent state discrimination using a closed-loop quantum measurement. Nature 446, 774 (2007).

[18] S. Chu. Nobel Lecture: The manipulation of neutral particles. Rev. Mod. Phys. 70, 685 (1998).

[19] C. N. Cohen-Tannoudji. Nobel Lecture: Manipulating atoms with photons. Rev. Mod. Phys. 70, 707 (1998).

[20] W. D. Phillips. Nobel Lecture: Laser cooling and trapping of neutral atoms. Rev. Mod. Phys. 70, 721 (1998).

[21] T. Hänsch and A. Schawlow. Cooling of gasses by laser radiation. Opt. Commun. 13, 68 (1975).

[22] D. J. Wineland and H. Dehmelt. Proposed $10^{14} \Delta \nu<\nu$ Laser Fluorescence Spectroscopy on TI $^{+}$Mono-Ion Oscillator III. Bull. Am. Phys. Soc. 20, 637 (1975).

[23] V. S. Letokhov, V. G. Minogin, and B. D. Pavlik. Cooling and trapping of atoms and molecules by a resonant laser field. Opt. Commun. 19, 72 (1976).

[24] A. Ashkin. Trapping of Atoms by Resonance Radiation Pressure. Phys. Rev. Lett. 40, 729 (1978).

[25] D. J. Wineland, R. E. Drullinger, and F. L. Walls. Radiation-Pressure Cooling of Bound Resonant Absorbers. Phys. Rev. Lett. 40, 1639 (1978).

[26] D. Wineland and W. Itano. Spectroscopy of a single $\mathrm{Mg}^{+}$ion. Phys. Lett. A $\mathbf{8 2 A}$, 75 (1981).

[27] W. D. Phillips and H. Metcalf. Laser Deceleration of an Atomic Beam. Phys. Rev. Lett. 48, 596 (1982).

[28] A. L. Migdall, J. V. Prodan, W. D. Phillips, T. H. Bergeman, and H. J. Metcalf. First Observation of Magnetically Trapped Neutral Atoms. Phys. Rev. Lett. 54, 2596 (1985). 
[29] E. Raab, M. Prentiss, A. Cable, S. Chu, and D. E. Pritchard. Trapping of Neutral Sodium Atoms with Radiation Pressure. Phys. Rev. Lett. 59, 2631 (1987).

[30] C. Monroe, W. Swann, H. Robinson, and C. Wieman. Very cold trapped atoms in a vapor cell. Phys. Rev. Lett. 65, 1571+ (1990).

[31] S. Chu, Bjorkholm, A. Ashkin, and A. Cable. Experimental observation of optically trapped atoms. Phys. Rev. Lett. 57, 314 (1986).

[32] P. D. Lett, R. N. Watts, C. I. Westbrook, W. D. Phillips, P. L. Gould, and H. J. Metcalf. Observation of Atoms Laser Cooled below the Doppler Limit. Phys. Rev. Lett. 61, 169 (1988).

[33] J. Dalibard and C. Cohen-Tannoudji. Dressed-Atom Approach to Atomic Motion in Laser Light: The Dipole Force Revisited. J. Opt. Soc. Am. B 2, 1707 (1985).

[34] J. Dalibard and C. Cohen-Tannoudji. Laser cooling below the Doppler limit by polarization gradients: simple theoretical models. J. Opt. Soc. Am. B 6, 2023 (1989).

[35] A. Aspect, E. Arimondo, R. Kaiser, N. Vansteenkiste, and C. Cohen-Tannoudji. Laser Cooling Below the One-Photon Recoil Energy by Velocity-Selective Coherent Population Trapping. Phys. Rev. Lett. 61, 826 (1988).

[36] Z. Hu and H. J. Kimble. Observation of a single atom in a magneto-optical trap. Opt. Lett. 19, 1889 (1994).

[37] D. Haubrich, H. Schadwinkel, F. Strauch, B. Ueberholz, R. Wynands, and D. Meschede. Observation of individual neutral atoms in magnetic and magnetooptical traps. Europhys. Lett. 34, 663 (1996).

[38] N. Schlosser, G. Reymond, I. Protsenko, and P. Grangier. Sub-poissonian loading of single atoms in a microscopic dipole trap. Nature 411, 1024 (2001).

[39] S. Kuhr, W. Alt, D. Schrader, M. Müller, V. Gomer, and D. Meschede. Deterministic Delivery of a Single Atom. Science 293, 278 (2001).

[40] I. P. Georges Reymond, Nicolas Schlosser and P. Grangier. Single-atom manipulations in a microscopic dipole trap. Phil. Trans. R. Soc. Lond. A 361, 1527 (2003).

[41] Q. A. Turchette, C. S. Wood, B. E. King, C. J. Myatt, D. Leibfried, W. M. Itano, C. Monroe, and D. J. Wineland. Deterministic Entanglement of Two Trapped Ions. Phys. Rev. Lett. 81, 3631 (1998).

[42] C. A. Sackett, D. Kielpinski, B. E. King, C. Langer, V. Meyer, C. J. Myatt, M. Rowe, Q. A. Turchette, W. M. Itano, D. J. Wineland, and C. Monroe. Experimental Entanglement of Four Particles. Nature 404, 256 (2000). 
[43] A. Rauschenbeutel, G. Nogues, S. Osnaghi, P. Bertet, M. Brune, J. Raimond, and S. Haroche. Step by step engineered many particle entanglement. Science 288, 2024 (2000).

[44] T. Udem. Timekeeping Light-insensitive optical clock. Nature 435, 291 (2005).

[45] E. T. Jaynes and F. W. Cummings. Comparison of Quantum and Semiclassical Radiation Theories with Application to the Beam Maser. Proc. IEEE 51, 89 (1963).

[46] D. Meschede, H. Walther, and G. Müller. One-Atom Maser. Phys. Rev. Lett. 54, 551 (1985).

[47] S. Haroche and D. Kleppner. Cavity Quantum Electrodynamics. Phys. Today 24-30 (1989).

[48] G. Rempe, H. Walther, and N. Klein. Observation of quantum collapse and revival in a one-atom maser. Phys. Rev. Lett. 58, 353 (1987).

[49] Y. Kaluzny, P. Goy, M. Gross, J. Raimond, and S. Haroche. Observation of SelfInduced Rabi Oscillations in Two-Level Atoms Excited Inside a Resonant Cavity: The Ringing Regime of Superradiance. Phys. Rev. Lett. 51, 1175 (1983).

[50] G. Rempe, R. J. Thompson, H. J. Kimble, and R. Lalezari. Measurement of ultralow losses in an optical interferometer. Opt. Lett. 17, 363 (1992).

[51] Y. Zhu, J. Gauthier, S. Morin, Q. Wu, H. Carmichael, and T. Mossberg. Vacuum Rabi splitting as a feature of linear-dispersion theory: Analysis and experimental observations. Phys. Rev. Lett. 64, 2499 (1990).

[52] J. Gripp, S. Mielke, and L. Orozco. Evolution of the vacuum Rabi peaks in a detuned Atoms-Cavity System. Phys. Rev. A 50, 3262 (1997).

[53] R. J. Thompson, G. Rempe, and H. J. Kimble. Observation of Normal-Mode Splitting for an Atom in an Optical Cavity. Phys. Rev. Lett. 68, 1132 (1992).

[54] J. J. Childs, K. An, M. S. Otteson, R. R. Dasari, and M. S. Feld. Normal Mode Line Shapes for Atoms in Standing-Wave Optical Resonators. Phys. Rev. Lett. 77, 2901 (1996).

[55] C. J. Hood, M. S. Chapman, T. W. Lynn, and H. J. Kimble. Real-time cavity QED with single atoms. Phys. Rev. Lett. 80, 4157 (1998).

[56] J. M. Raimond, T. Meunier, P. Bertet, S. Gleyzes, P. Maioli, A. Auffeves, G. Nogues, M. Brune, and S. Haroche. Probing a quantum field in a photon box. J. Phys. B 38, S535 (2005). 
[57] S. Gleyzes, S. Kuhr, C. Guerlin, J. Bernu, S. Delglise, U. B. Hoff, M. Brune, J.-M. Raimond, and S. Haroche. Quantum jumps of light recording the birth and death of a photon in a cavity. Nature 446, 297 (2007).

[58] G. Nogues, A. Rauschenbeutel, S. Osnaghi, M. Brune, J. M. Raimond, and S. Haroche. Seeing a single photon without destroying it. Nature 400, 239 (1999).

[59] S. Haroche and J. M. Raimond. Exploring the Quantum (Oxford Univ. Press, 2006).

[60] J. M. Raimond, M. Brune, and S. Haroche. Colloquium: Manipulating quantum entanglement with atoms and photons in a cavity. Rev. Mod. Phys. 73, 565 (2001).

[61] P. Bertet, S. Osnaghi, A. Rauschenbeutel, G. Nogues, A. Auffeves, M. Brune, J. M. Raimond, and S. Haroche. A complementarity experiment with an interferometer at the quantum-classical boundary. Nature 411, 166 (2001).

[62] A. Kuhn, M. Hennrich, and G. Rempe. Deterministic single-photon source for distributed quantum networking. Phys. Rev. Lett. 89, 067901 (2002).

[63] J. McKeever, A. Boca, A. D. Boozer, R. Miller, J. R. Buck, A. Kuzmich, and H. J. Kimble. Deterministic Generation of Single Photons from One Atom Trapped in a Cavity. Science 303, 1992 (2004).

[64] T. Wilk, S. C. Webster, H. P. Specht, G. Rempe, and A. Kuhn. PolarizationControlled Single Photons. Phys. Rev. Lett. 98 (2007).

[65] A. D. Boozer, A. Boca, R. Miller, T. E. Northup, and H. J. Kimble. Reversible state transfer between light and a single trapped atom. arXiv: quant-ph/0702248v1 (2007).

[66] M. Hijlkema, B. Weber, H. P. Specht, S. C. Webster, A. Kuhn, and G. Rempe. A single-photon server with just one atom. Nat. Phys. 3, 253 (2007).

[67] E. Knill, R. Laflamme, and G. J. Milburn. A scheme for efficient quantum computing with linear optics. Nature 409, 46 (2001).

[68] J. I. Cirac, P. Zoller, H. J. Kimble, and H. Mabuchi. Quantum State Transfer and Entanglement Distribution Among Distant Nodes in a Quantum Network. Phys. Rev. Lett. 78, 3221 (1997).

[69] H.-J. Briegel, W. Dür, J. I. Cirac, and P. Zoller. Quantum Repeaters: The Role of Imperfect Local Operations in Quantum Communication. Phys. Rev. Lett. 81, 5932 (1998).

[70] J. F. Sherson, H. Krauter, R. K. Olsson, B. Julsgaard, K. Hammerer, I. Cirac, and E. S. Polzik. Quantum teleportation between light and matter. Nature 443, 557 (2006). 
[71] A. Wallraff, D. I. Schuster, A. Blais, L. Frunzio, R. S. Huang, J. Majer, S. Kumar, S. M. Girvin, and R. J. Schoelkopf. Circuit Quantum Electrodynamics: Coherent Coupling of a Single Photon to a Cooper Pair Box. Nature 431, 162 (2004).

[72] I. Chiorescu, P. Bertet, K. Semba, Y. Nakamura, C. J. P. M. Harmans, and J. E. Mooij. Coherent dynamics of a flux qubit coupled to a harmonic oscillator. Nature 431, 159 (2004).

[73] T. Yoshie, A. Scherer, J. Hendrickson, G. Khitrova, H. M. Gibbs, G. Rupper, C. Ell, O. B. Shchekin, and D. G. Deppe. Vacuum Rabi splitting with a single quantum dot in a photonic crystal nanocavity. Nature 432, 200 (2004).

[74] K. Hennessy, A. Badolato, M. Winger, D. Gerace, M. Atatüre, S. Gulde, S. Fält, E. L. Hu, and A. Imamoglu. Quantum nature of a strongly coupled single quantum dot-cavity system. Nature 445, 1 (2007).

[75] J. P. Reithmaier, G. Sek, A. Loffler, C. Hofmann, S. Kuhn, S. Reitzenstein, L. V. Keldysh, V. D. Kulakovskii, T. L. Reinecke, and A. Forchel. Strong coupling in a single quantum dot-semiconductor microcavity system. Nature 432, 197 (2004).

[76] E. Peter, P. Senellart, D. Martrou, A. Lemaitre, J. Hours, J. M. Gerard, and J. Bloch. Exciton-Photon Strong-Coupling Regime for a Single Quantum Dot Embedded in a Microcavity. Phys. Rev. Lett. 95, 067401 (2005).

[77] B. C. Sanders, H. J. Carmichael, and B. F. Wielinga. Photon correlation spectroscopy of the Jaynes-Cummings system. Phys. Rev. A 55, 1358 (1997).

[78] M. Brune, F. Schmidt-Kaler, A. Maali, J. Dreyer, E. Hagley, J.-M. Raimond, and S. Haroche. Quantum Rabi Oscillation: A Direct Test of Field Quantization in a Cavity. Phys. Rev. Lett. 76, 1800 (1996).

[79] G. Khitrova, H. M. Gibbs, M. Kira, S. W. Koch, and A. Scherer. Vacuum Rabi splitting in semiconductors. Nat. Phys. 2, 81 (2006).

[80] D. Sanvitto, F. P. Laussy, F. Bello, D. M. Whittaker, A. M. Fox, M. S. Skolnick, A. Tahraoui, P. W. Fry, and M. Hopkinson. Single-photon nonlinearity of a semiconductor quantum dot in a cavity. arXiv: cond-mat/0612034 (2006).

[81] K. M. Birnbaum, A. Boca, R. Miller, A. D. Boozer, T. E. Northup, and H. J. Kimble. Photon blockade in an optical cavity with one trapped atom. Nature 436, 87 (2005).

[82] J. Ye, D. W. Vernooy, and H. J. Kimble. Trapping of Single Atoms in Cavity QED. Phys. Rev. Lett. 83, 4987 (1999).

[83] A. C. Doherty, T. W. Lynn, C. J. Hood, and H. J. Kimble. Trapping of single atoms with single photons in cavity QED. Phys. Rev. A 63, 013401 (2000). 
[84] P. W. H. Pinkse, T. Fischer, P. Maunz, and G. Rempe. Trapping an Atom with Single Photons. Nature 404, 365 (2000).

[85] G. Hechenblaikner, M. Gangl, P. Horak, and H. Ritsch. Cooling an Atom in a Weakly Driven High-Q Cavity. Phys. Rev. A 58, 3030 (1998).

[86] K. Murr, P. Maunz, P. W. H. Pinkse, T. Puppe, I. Schuster, D. Vitali, and G. Rempe. Momentum diffusion for coupled atom-cavity oscillators. Phys. Rev. A 74, $043412(2006)$.

[87] P. Maunz. Cavity cooling and spectroscopy of a bound atom-cavity system. Ph.D. thesis, Max-Planck-Institut für Quantenoptik und Technische Universität München (2004).

[88] P. Maunz, T. Puppe, I. Schuster, N. Syassen, P. W. H. Pinkse, and G. Rempe. Cavity cooling of a single atom. Nature 428, 50 (2004).

[89] T. W. Mossberg, M. Lewenstein, and D. J. Gauthier. Trapping and cooling of atoms in a vacuum perturbed in a frequency-dependent manner. Phys. Rev. Lett. 67, 1723 (1991).

[90] M. Lewenstein and L. Roso. Cooling of atoms in colored vacua. Phys. Rev. A 47, 3385 (1993).

[91] P. Horak, G. Hechenblaikner, K. M. Gheri, H. Stecher, and H. Ritsch. CavityInduced Atom Cooling in the Strong Coupling Regime. Phys. Rev. Lett. 79, 4974 (1997).

[92] V. Vuletić and S. Chu. Laser cooling of atoms, ions, or molecules by coherent scattering. Phys. Rev. Lett. 84, 3787 (2000).

[93] A. Griessner, D. Jaksch, and P. Zoller. Cavity-assisted nondestructive laser cooling of atomic qubits. J. Phys. B 37, 1419 (2004).

[94] P. Maunz, T. Puppe, I. Schuster, N. Syassen, P. W. H. Pinkse, and G. Rempe. Normal-mode spectroscopy of a single bound atom-cavity system. Phys. Rev. Lett. 94, 033002 (2005).

[95] J. McKeever, J. R. Buck, A. D. Boozer, A. Kuzmich, H.-C. Nagerl, D. M. StamperKurn, and H. J. Kimble. State-Insensitive Cooling and Trapping of Single Atoms in an Optical Cavity. Phys. Rev. Lett. 90, 133602 (2003).

[96] T. Puppe, I. Schuster, A. Grothe, A. Kubanek, K. Murr, P. W. H. Pinkse, and G. Rempe. Trapping and observing single atoms in the dark. arXiv: quantph/0702162 (2007).

[97] S. Nußmann, K. Murr, M. Hijlkema, B. Weber, A. Kuhn, and G. Rempe. Vacuumstimulated cooling of single atoms in three dimensions. Nat. Phys. 1, 122 (2005). 
[98] H. Mabuchi, Q. A. Turchette, M. S. Chapman, and H. J. Kimble. Real-Time Detection of Individual Atoms Falling Through a High-Finesse Optical Cavity. Opt. Lett. 21, 1393 (1996).

[99] P. Münstermann, T. Fischer, P. W. H. Pinkse, and G. Rempe. Single Slow Atoms from an Atomic Fountain Observed in a High-Finesse Optical Cavity. Opt. Commun. 159, 63 (1999).

[100] G. Rempe. One atom in an optical cavity: spatial resolution beyond the standard diffraction limit. Appl. Phys. B 60, 233 (1995).

[101] C. J. Hood, T. Lynn, A. C. Doherty, A. S. Parkins, and H. J. Kimble. The AtomCavity Microscope: Single Atoms Bound in Orbit by Single Photons. Science 287, 1477 (2000).

[102] T. Fischer, P. Maunz, P. W. H. Pinkse, T. Puppe, and G. Rempe. Feedback on the motion of a single atom in an optical cavity. Phys. Rev. Lett. 88, 163002 (2002).

[103] P. Horak, H. Ritsch, T. Fischer, P. Maunz, T. Puppe, P. Pinkse, and G. Rempe. An optical kaleidoscope using a single atom. Phys. Rev. Lett. 88, 043601 (2002).

[104] P. Maunz, T. Puppe, T. Fischer, P. Pinkse, and G. Rempe. Emission pattern of an atomic dipole in a high-finesse optical cavity. Opt. Lett. 28, 46 (2003).

[105] T. Puppe, P. Maunz, T. Fischer, P. Pinkse, and G. Rempe. Single-atom trajectories in higher-order transverse modes of a high-finesse optical cavity. Phys. Scr. T112, 7 (2004).

[106] T. Fischer. Dynamik einzelner Atome in einem optischen Resonator hoher Finesse. Diplomathesis, Universität Konstanz (1998).

[107] M. Tavis and F. W. Cummings. Exact Solution for an N-Molecule-Radiation-Field Hamiltonian. Phys. Rev. 170, 379 (1968).

[108] H. J. Carmichael and B. C. Sanders. Multiatom effects in cavity QED with atomic beams. Phys. Rev. A 60, 2497 (1999).

[109] K. Murr. On the suppression of the diffusion and the quantum nature of a cavity mode. Optical bistability: Forces and friction in driven cavities. J. Phys. B 36, 2515 (2003).

[110] L. A. Lugiato. Theory of optical bistability. E. Wolf, (editor) Progress in optics, vol. 21, 69-216 (Elsevier Science Publishers, B. V., 1984).

[111] T. Fischer. Controlling the motion of an atom in an optical cavity. Ph.D. thesis, Max-Planck-Institut für Quantenoptik und Technische Universität München (2002). 
[112] C. Cohen-Tannoudji. Atomic Motion in Laser Light. J. Dalibard, J. M. Raimond, and J. Zinn-Justin, (editors) Fundamental Systems in Quantum Optics, Les Houches, Session LIII, 1990, no. 53 in Les Houches Lectures, 1 (Elsevier Science, North-Holland, 1992).

[113] R. Grimm, M. Weidemüller, and Y. B. Ovchinnikov. Optical dipole traps for neutral atoms. Adv. At. Mol. Opt. Phys. 42, 95 (2000).

[114] A. W. Grothe. Blau verstimmte Resonatormoden als Dipolfalle fr einzelne Atome. Master's thesis, Max-Planck-Institut fr Quantenoptik und Technische Universitt Mnchen (2005).

[115] P. Maunz. Photon induced motion of single atoms in a high finesse cavity. Master's thesis, Universität Konstanz (1999).

[116] P. Münstermann, T. Fischer, P. Maunz, P. W. H. Pinkse, and G. Rempe. Dynamics of Single-Atom Motion Observed in a High-Finesse Cavity. Phys. Rev. Lett. 82, 3791 (1999).

[117] T. A. Savard, K. M. O'Hara, and J. E. Thomas. Laser-noise-induced heating in far-off resonance optical traps. Phys. Rev. A 56, R1095 (1997).

[118] S. Nußmann, M. Hijlkema, B. Weber, F. Rohde, G. Rempe, and A. Kuhn. SubMicron Positioning of Single Atoms in Micro Cavities. Phys. Rev. Lett. 95, 173602 (2005).

[119] J. A. Sauer, K. M. Fortier, M. S. Chang, C. D. Hamley, and M. S. Chapman. Cavity QED with optically transported atoms. Phys. Rev. A 69, 051804(R) (2004).

[120] M. Elbs. Realisierung einer magnetooptischen Falle. Diplomathesis, Universität Konstanz (1994).

[121] R. Muscheler. Realisation einer Fontäne optisch gekühlter Atome. Diplomathesis, Universität Konstanz (1997).

[122] P. Münstermann. Aufbau und Charakterisierung eines optischen Resonators hoher Finesse. Diplomathesis, Universität Konstanz (1995).

[123] T. Puppe. Lichtkräfte auf Atome in einem optischen Resonator. Diplomathesis, Universität Konstanz (2000).

[124] B. E. A. Saleh and M. C. Teich. Fundamentals of photonics (Wiley, 1991).

[125] C. J. Hood and H. J. Kimble. Characterization of high-finesse mirrors: Loss, phase shifts, and mode structure in an optical cavity. Phys. Rev. A 64, 033804 (2001).

[126] K. An, C. Yang, R. R. Dasari, and M. S. Feld. Cavity ring-down technique and its application to the measurement of ultraslow velocities. Opt. Lett. 20, 1068 (1995). 
[127] U. Ernst. Stabilisierung der Differenz zweier Laserfrequenzen mit Hilfe eines optischen Resonators. Diplomathesis, Universität Konstanz (1995).

[128] R. Drever, J. Hall, F. Kowalski, J. Hough, G. Ford, A. Munley, and H. Ward. Laser phase and frequency stabilization using an optical resonator. Appl. Phys. B 31, 97 (1983).

[129] A. Schoof, J. Grünert, S. Ritter, and A. Hemmerich. Reducing the linewidth of a diode laser below $30 \mathrm{~Hz}$ by stabilization to a reference cavity with a finesse above $10^{5}$. Opt. Lett. 26, 1562 (2001).

[130] A. D. Boozer, A. Boca, R. Miller, T. E. Northup, and H. J. Kimble. Cooling to the Ground State of Axial Motion for One Atom Strongly Coupled to an Optical Cavity. Phys. Rev. Lett. 97, 083602 (2006).

[131] P. Horak, B. G. Klappauf, A. Haase, R. Folman, J. Schmiedmayer, P. Domokos, and E. A. Hinds. Towards single-atom detection on a chip. Phys. Rev. A 67, 043806 (2003).

[132] M. Trupke, E. A. Hinds, S. Eriksson, E. A. Curtis, Z. Moktadir, E. Kukharenka, and M. Kraft. Microfabricated high-finesse optical cavity with open access and small volume. Appl. Phys. Lett. 87, 211106 (2005).

[133] T. Steinmetz, Y. Colombe, D. Hunger, T. W. Hänsch, A. Balocchi, R. J. Warburtona, and J. Reichel. Stable fiber-based Fabry-Pérot cavity. Appl. Phys. Lett. 89, 111110 (2006).

[134] I. Teper, Y.-J. Lin, and V. Vuletić. Resonator-Aided Single-Atom Detection on a Microfabricated Chip. Phys. Rev. Lett. 97, 023002 (2006).

[135] A. Öttl, S. Ritter, M. Köhl, and T. Esslinger. Correlations and Counting Statistics of an Atom Laser. Phys. Rev. Lett. 95, 090404 (2005).

[136] I. Wilson-Rae, N. Nooshi, W. Zwerger, and T. J. Kippenberg. Theory of ground state cooling of a mechanical oscillator using dynamical back-action. arXiv: condmat/0702113 (2007).

[137] S. Nußmann. Kühlen und Positionieren eines Atoms in einem optischen Resonator. Ph.D. thesis, Max-Planck Institut für Quantenoptik (2005).

[138] A. Boca, R. Miller, K. M. Birnbaum, A. D. Boozer, J. McKeever, and H. J. Kimble. Observation of the Vacuum Rabi Spectrum for One Trapped Atom. Phys. Rev. Lett. 93, 233603 (2004).

[139] T. Puppe, I. Schuster, P. Maunz, K. Murr, P. W. H. Pinkse, and G. Rempe. Light force fluctuations in a strongly coupled atom-cavity system. arXiv: quantph/0702087 (2007). 
[140] N. Syassen. Linear and nonlinear spectroscopy of a strongly-coupled atom-cavity system. Diplomathesis, Max-Planck-Institut für Quantenoptik und Technische Universität München (2003).

[141] G. Varada, M. Kumar, and G. Agarwal. Quantum effects of the atom-cavity interaction on four-wave mixing. Opt. Commun. 62, 328 (1987).

[142] E. Haiver, S. Novsett, and G. Wanner. Solving ordinary differential equations, vol. I of Springer Series in Computational Mathematics 8 (Springer Verlag, 1987).

[143] R. Quadt, M. Collett, and D. Walls. Measurement of atomic motion in a standing light field by homodyne detection. Phys. Rev. Lett. 74, 351 (1995).

[144] F. Diedrich, J. C. Bergquist, W. M. Itano, and D. J. Wineland. Laser Cooling to the Zero-Point Energy of Motion. Phys. Rev. Lett. 62, 403 (1989).

[145] C. Monroe, D. M. Meekhof, B. E. King, S. R. Jefferts, W. M. Itano, and D. J. Wineland. Resolved-Sideband Raman Cooling of a bound atom to the $3 D$ zeropoint energy. Phys. Rev. Lett. 75, 4011 (1995).

[146] C. Monroe. Quantum information processing with atoms and photons. Nature 416, 238 (2002).

[147] D. W. Vernooy and H. J. Kimble. Well-dressed states for wave-packet dynamics in cavity QED. Phys. Rev. A 56, 4287 (1997).

[148] R. Miller, T. E. Northup, K. M. Birnbaum, A. Boca, A. D. Boozer, and H. J. Kimble. Trapped atoms in cavity QED: coupling quantized light and matter. J. Phys. B 38, S551 (2005).

[149] A. S. Parkins and H. J. Kimble. Quantum state transfer between motion and light. J. Opt. B: Quant. Semiclass. Opt. 1, 496 (1999).

[150] A. S. Parkins and H. J. Kimble. Position-Momentum Einstein-Podolsky-Rosen State of Distantly Separated Trapped Atoms. Phys. Rev. A 61, 052104 (2000).

[151] M. J. Holland, D. F. Walls, and P. Zoller. Quantum nondemolition measurements of photon number by atomic beam deflection. Phys. Rev. Lett. 67, 1716 (1991).

[152] W. Ren and H. J. Carmichael. Spontaneous emission in a standing-wave cavity: Quantum-mechanical center-of-mass motion. Phys. Rev. A 51, 752 (1995).

[153] A. Herkommer, H. Carmichael, and W. Schleich. Localization of an atom by homodyne measurement. Quant. Semiclass. Opt. 8, 189 (1996).

[154] A. Doherty, A. Parkins, S. Tan, and D. Walls. Motional states of atoms in cavity QED. Phys. Rev. A 57, 4804 (1998). 
[155] D. W. Vernooy and H. J. Kimble. Quantum structure and dynamics for atom galleries. Phys. Rev. A 55, 1239 (1997).

[156] J. Reichel. Microchip traps and Bose Einstein condensation. Appl. Phys. B 74, 469 (2002).

[157] D. Müller, D. Z. Anderson, R. J. Grow, P. D. D. Schwindt, and E. A. Cornell. Guiding Neutral Atoms Around Curves with Lithographically Patterned CurrentCarrying Wires. Phys. Rev. Lett. 83, 5194 (1999).

[158] N. H. Dekker, C. S. Lee, V. Lorent, J. H. Thywissen, S. P. Smith, M. Drndić, R. M. Westervelt, and M. Prentiss. Guiding Neutral Atoms on a Chip. Phys. Rev. Lett. 84, 1124 (2000).

[159] R. Folman, P. Krüger, J. Schmiedmayer, J. Denschlag, and C. Henkel. Microscopic Atom Optics: From Wires to an Atom Chip. Adv. At. Mol. Opt. Phys. 48, 263 (2002).

[160] D. Cassettari, B. Hessmo, R. Folman, T. Maier, and J. Schmiedmayer. Beam Splitter for Guided Atoms. Phys. Rev. Lett. 85, 5483 (2000).

[161] T. Schumm, S. Hofferberth, L. M. Andersson, S. Wildermuth, S. Groth, I. BarJoseph, J. Schmiedmayer, and P. Krüger. Matter-wave interferometry in a double well on an atom chip. Nat. Phys. 1, 57 (2005).

[162] W. Hänsel, P. Hommelhoff, T. W. Hänsch, and J. Reichel. Bose-Einstein condensation on a microelectronic chip. Nature 413, 498 (2001).

[163] C. D. J. Sinclair, E. A. Curtis, L. I. Garcia, J. A. Retter, B. V. Hall, S. Eriksson, B. E. Sauer, and E. A. Hinds. Bose-Einstein condensation on a permanent-magnet atom chip. Phys. Rev. A 72, 031603 (2005).

[164] W. Hänsel, J. Reichel, P. Hommelhoff, and T. W. Hänsch. Trapped-atom interferometer in a magnetic microtrap. Phys. Rev. A 64, 063607 (2001).

[165] P. Treutlein, T. Steinmetz, Y. Colombe, B. Lev, P. Hommelhoff, J. Reichel, M. Greiner, O. Mandel, A. Widera, T. Rom, I. Bloch, and T. W. Hänsch. Quantum Information Processing in Optical Lattices and Magnetic Microtraps. Fortschr. Phys. 54, 702 (2006).

[166] K. J. Vahala. Optical microcavities. Nature 424, 839 (2003).

[167] M. Hossein-Zadeh and K. J. Vahala. Free ultra-high- $Q$ microtoroid: a tool for designing photonic devices. Opt. Express 15, 166 (2007).

[168] D. K. Armani, T. J. Kippenberg, S. M. Spillane, and K. J. Vahala. Ultra-high-Q toroid microcavity on a chip. Nature 421, 925 (2003). 
[169] S. M. Spillane, T. J. Kippenberg, O. J. Painter, and K. J. Vahala. Ideality in a Fiber-Taper-Coupled Microresonator System for Application to Cavity Quantum Electrodynamics. Phys. Rev. Lett. 91, 043902 (2003).

[170] M. Rosenblit, P. Horak, S. Helsby, and R. Folman. Single-atom detection using whispering-gallery modes of microdisk resonators. Phys. Rev. A 70, 053808 (2004).

[171] T. Aoki, B. Dayan, E. Wilcut, W. P. Bowen, A. S. Parkins, T. J. Kippenberg, K. J. Vahala, and H. J. Kimble. Observation of strong coupling between one atom and a monolithic microresonator. Nature 443, 671 (2007).

[172] A. Schliesser, P. Del'haye, N. Nooshi, K. J. Vahala, and T. J. Kippenberg. Radiation Pressure Cooling of a Micromechanical Oscillator Using Dynamical Backaction. Physical Review Letters 97 (2006).

[173] I. Dotsenko, W. Alt, M. Khudaverdyan, S. Kuhr, D. Meschede, Y. Miroshnychenko, D. Schrader, and A. Rauschenbeutel. Submicrometer Position Control of Single Trapped Neutral Atoms. Phys. Rev. Lett. 95, 033002 (2005).

[174] Y. Miroshnychenko, W. Alt, I. Dotsenko, L. Forster, M. Khudaverdyan, D. Meschede, S. Reick, and A. Rauschenbeutel. Inserting Two Atoms into a Single Optical Micropotential. Phys. Rev. Lett. 97, 243003 (2006).

[175] J. McKeever, J. R. Buck, A. D. Boozer, and H. J. Kimble. Determination of the number of atoms trapped in an optical cavity. Phys. Rev. Lett. 93, 143601 (2004).

[176] T. Wilk, S. C. Webster, G. Rempe, and A. Kuhn (2007). To be published.

[177] M. Keller, B. Lange, K. Hayasaka, W. Lange, and H. Walther. Continuous generation of single photons with controlled waveform in an ion-trap cavity system. Nature 431, 1075 (2004).

[178] N. Gisin, G. Ribordy, W. Tittel, and H. Zbinden. Quantum Cryptography. Rev. Mod. Phys. 74, 145 (2002).

[179] L. Tian and H. Carmichael. Quantum trajectory simulations of the two-state behavior of an optical cavity containing one atom. Phys. Rev. A 46, R6801 (1992).

[180] Q. A. Turchette, R. J. Thompson, and H. J. Kimble. One-dimensional atoms. Appl. Phys. B 60, S1 (1995).

[181] G. T. Foster, L. A. Orozco, H. M. Castro-Beltran, and H. J. Carmichael. Quantum State Reduction and Conditional Time Evolution of Wave-Particle Correlations in Cavity QED. Phys. Rev. Lett. 85, 3149 (2000).

[182] S. L. Mielke, G. T. Foster, and L. A. Orozco. Nonclassical Intensity Correlations in Cavity QED. Phys. Rev. Lett. 80, 3948 (1998).

[183] L. Horvath and H. J. Carmichael. Effect of atomic beam alignment on photon correlation measurements in cavity QED. arXiv: 0704.1686v1 (2007). 



\section{Publications}

T. Puppe, I. Schuster, P. Maunz, K. Murr, P.W.H. Pinkse, and G. Rempe.

Light force fluctuations in a strongly coupled atom-cavity system.

arXiv:quant-ph/0702087.

T. Puppe, I. Schuster, A. Grothe, A. Kubanek, K. Murr, P.W.H. Pinkse, and G. Rempe. Trapping and observing single atoms in the dark.

arXiv:quant-ph/0702162.

K. Murr, P. Maunz, P.W.H. Pinkse, T. Puppe, I. Schuster, D. Vitali, and G. Rempe. Momentum diffusion for coupled atom-cavity oscillators.

Physical Review A 74, 043412 (2006).

K. Murr, S. Nußmann, T. Puppe, M. Hijlkema, B. Weber, S. C. Webster, A. Kuhn, and G. Rempe. Three-dimensional cavity cooling and trapping in an optical lattice.

Physical Review A 73, 063415 (2006).

P. Maunz, T. Puppe, I. Schuster, N. Syassen, P.W.H. Pinkse, and G. Rempe.

Normal-mode spectroscopy of a single-bound-atom-cavity system.

Physical Review Letters 94, 033002 (2005).

P. Maunz, T. Puppe, I. Schuster, N. Syassen, P.W.H. Pinkse, and G. Rempe.

Cavity cooling of a single atom.

Nature 428, 50-52 (2004).

T. Puppe, P. Maunz, T. Fischer, P.W.H. Pinkse, and G. Rempe.

Single-atom trajectories in higher-order transverse modes of a high-finesse optical cavity. Physica Scripta, T112, p 7-12 (2004).

P. Maunz, T. Puppe, T. Fischer, P. W. H. Pinkse, and G. Rempe.

Emission pattern of an atomic dipole in a high-finesse optical cavity.

Optics Letters 28, 46 (2003).

T. Fischer, P. Maunz, P. W. H. Pinkse, T. Puppe and G. Rempe.

Feedback on the Motion of a Single Atom in an Optical Cavity.

Physical Review Letters 88, 163002 (2002). 
P. Horak, H. Ritsch, T. Fischer, P. Maunz, T. Puppe, P.W.H. Pinkse and G. Rempe. An optical kaleidoscope using a single atom.

Physical Review Letters 88, 043601 (2002).

T. Fischer, P. Maunz, T. Puppe, P.W.H. Pinkse and G. Rempe.

Collective light forces on atoms in a high-finesse cavity.

New Journal of Physics 3, (2001).

P.W.H. Pinkse, T. Fischer, P. Maunz, T. Puppe, and G. Rempe.

How to catch an atom with single photons.

Journal of Modern Optics 47, 2769-2787 (2000). 


\section{Danksagung}

Zuallererst danke ich meinem Doktorvater Gerhard Rempe für die Möglichkeit meine Forschungsarbeit an diesem interessanten Experiment durchzuführen. Insbesondere bedanke ich mich für das Vertrauen in die Idee der blauen Falle und dafür, dass ich die Zeit bekommen habe diese am Experiment zu realisieren.

Ein Experiment dieser Komplexität ist nur durch Beiträge vieler Mitarbeiter möglich, bedanken möchte ich mich deshalb bei allen, die zum Gelingen dieser Arbeit beigetragen haben, angefangen bei jenen, die das Experiment in der Konstanzer Zeit entwickelt haben. Pepijn Pinkse danke ich für seine langjährige Unterstützung im Laufe der Arbeit. Meinen Vorgängern Thomas Fischer und Peter Maunz von denen ich viel über das Experiment gelernt habe danke ich für die interessante gemeinsame Zeit beim Wiederaufbau in Garching sowie den darauf folgenden Messungen. Bei Ingrid Schuster bedanke ich mich für ihren großen Einsatz für das Experiment. Ihr und Alexander Kubanek danke ich außerdem für ihre Unterstützung bei den Messungen zur blauen Falle. Niels Syassen danke ich für die verrückte Zeit bei der Arbeit an der Zweiphotonenspektroskopie und Alexander Grothe für seine Unterstützung beim Aufbau der blauen Falle. Ein spezieller Dank gilt all jenen, die mich mit ihren Kommentaren bei der Anfertigung dieser Arbeit unterstützt haben.

Einen herzlichen Dank den Technikern Sepp Bayerl, Franz Denk, Helmuth Stehbeck und Tom Wiesmeier für ihre schnelle und kompetente Hilfe bei der Lösung der technischen Herausforderungen am Experiment.

Allen anderen Mitgliedern der Arbeitsgruppe danke ich für das gute Arbeitsklima und den regen Wissens- und Materialtransfer. Ein besonderer Dank gilt denen, die im Interesse der Arbeitsgruppe zusätzliche Aufgaben übernehmen.

Ganz besonders bedanken möchte ich mich bei meinen Freunden und bei meiner Familie, ohne deren Anteilnahme und Unterstützung diese Arbeit nicht möglich gewesen wäre. 